\title{
LOCAL DIGITAL CONTROL OF POWER ELECTRONIC CONVERTERS IN A DC MICROGRID BASED ON A-PRIORI DERIVATION OF SWITCHING SURFACES
}

Bibaswan Banerjee

Michigan Technological University

Follow this and additional works at: https://digitalcommons.mtu.edu/etds

Part of the Power and Energy Commons

Copyright 2013 Bibaswan Banerjee

Recommended Citation

Banerjee, Bibaswan, "LOCAL DIGITAL CONTROL OF POWER ELECTRONIC CONVERTERS IN A DC MICROGRID BASED ON A-PRIORI DERIVATION OF SWITCHING SURFACES", Dissertation, Michigan Technological University, 2013.

https://doi.org/10.37099/mtu.dc.etds/651

Follow this and additional works at: https://digitalcommons.mtu.edu/etds

Part of the Power and Energy Commons 
LOCAL DIGITAL CONTROL OF POWER ELECTRONIC CONVERTERS IN A DC MICROGRID BASED ON A-PRIORI DERIVATION OF SWITCHING SURFACES

By

Bibaswan Banerjee

\begin{abstract}
A DISSERTATION
Submitted in partial fulfillment of the requirements for the degree of DOCTOR OF PHILOSOPHY

In Electrical Engineering
\end{abstract}

MICHIGAN TECHNOLOGICAL UNIVERSITY

2013

(C) 2013 Bibaswan Banerjee 

This dissertation has been approved in partial fulfillment of the requirements for the Degree of DOCTOR OF PHILOSOPHY in Electrical Engineering.

Department of Electrical and Computer Engineering

Dissertation Advisor: Dr. Wayne W. Weaver.

Committee Member: Prof. Leonard J. Bohmann.

Committee Member: Prof. Gordon G. Parker.

Committee Member: Prof. Duane J. Bucheger.

Department Chair: Prof. Daniel R. Fuhrmann. 



\section{To my wife Aparajita, all my teachers and friends}

without whose support and help this work wouldn't have been possible. 



\section{Contents}

List of Figures $\ldots \ldots \ldots \ldots \ldots \ldots \ldots \ldots \ldots \ldots \ldots \ldots \ldots$

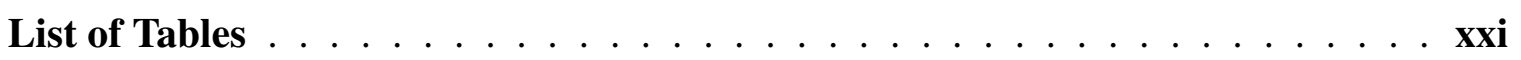

Acknowledgments $\ldots \ldots \ldots \ldots \ldots \ldots \ldots \ldots \ldots \ldots \ldots$. . . . . . . . . . . . . . . .

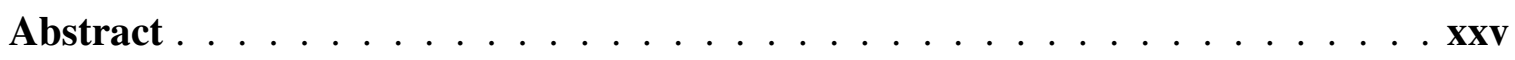

1 Introduction and Background $\ldots \ldots \ldots \ldots \ldots$

1.1 Motivation . . . . . . . . . . . . . . . . . . . . 1

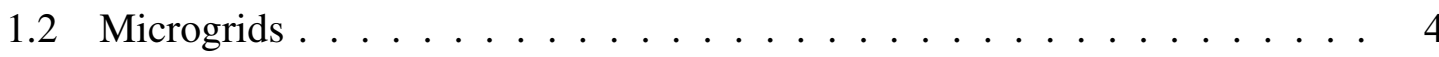

1.3 Game-theoretic control . . . . . . . . . . . . . . . . . 6

1.4 Sliding mode control $\ldots \ldots \ldots \ldots$

1.5 Digital control in power electronics . . . . . . . . . . . . . . . 10

1.6 Dissertation organization $\ldots \ldots \ldots \ldots$

2 Game-theoretic geometric manifolds in de microgrids . . . . . . . . . . 15

2.1 Generalized game-theoretic control manifolds in a dc microgrid . . . . . 16

2.1.1 Derivation of the game-theoretic control manifold . . . . . . . . 16 
2.1.1.1 Formulation of the game-theoretic control problem . . . . 16

2.1.1.2 Solution to the game-theoretic control problem . . . . . 23

2.1.2 Construction of the game-theoretic manifold in the energy-power

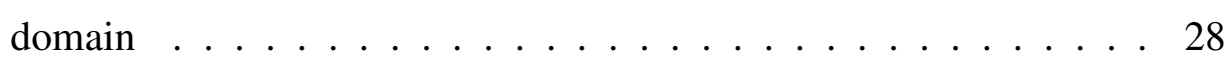

2.1.3 Conversion of the game-theoretic manifolds to the voltage-current

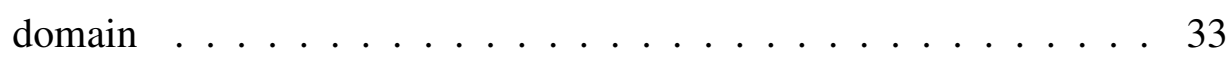

2.2 Simulation results . . . . . . . . . . . . . . . 38

2.3 Limits of applicability of the method and response in unknown situations . . 41

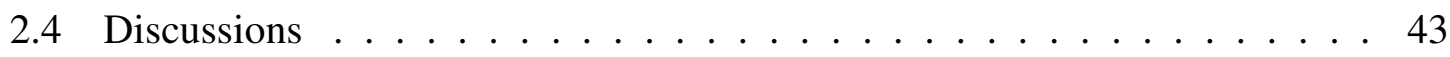

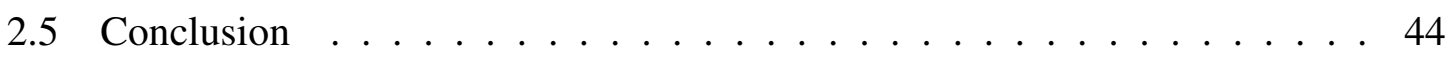

3 Digital sliding mode hysteretic control in a dc-dc boost converter . . . . . . . 45

3.1 Implementation of the digital hysteretic sliding mode controller in real time 46

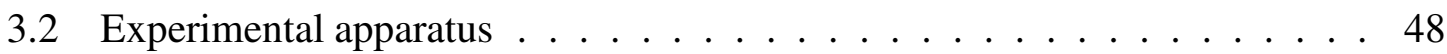

3.3 Memory resolution analysis . . . . . . . . . . . . . . . 49

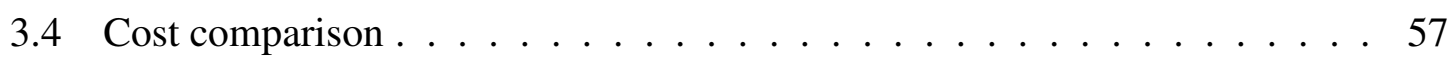

3.5 Simulation and hardware results for implementation of the digital sliding mode control strategy in a dc-dc boost converter . . . . . . . . . . 59

3.5.1 Implementation of the digital sliding mode control strategy of a straight line surface ................... . . . . . . . . .

3.5.1.1 Stability analysis for the straight line surface . . . . . 62

3.5.1.2 Simulation results for the straight line reference surface . 65 
3.5.1.3 Experimental results for the straight line reference surface 66

3.5.1.4 Analysis of the ADC latency for the straight line reference surface . . . . . . . . . . . . . 696

3.5.2 Linear sloped surface .................... 71

3.5.2.1 Stability analysis for the linear sloped surface . . . . . 73

3.5.2.2 Simulation results for the sloped reference surface . . . 76

3.5.2.3 Experimental results for the sloped reference surface . . 78

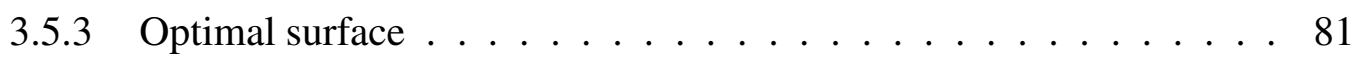

3.5.3.1 Derivation of the optimal reference surface . . . . . 82

3.5.3.2 Stability analysis for the non-linear optimal surface . . . . 86

3.5.3.3 Simulation results for the optimal reference surface . . . 89

3.5.3.4 Experimental results for the optimal reference surface . . 91

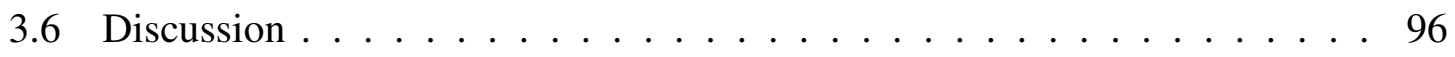

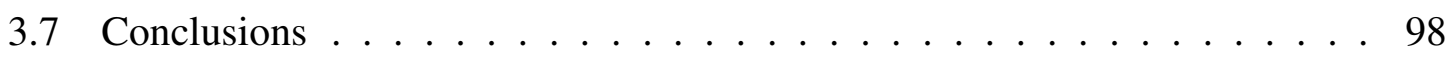

4 Digital sliding mode hysteretic controller in a dc microgrid . . . . . . . . . 99

4.1 Derivation of the geometric surface and implementation of the digital controller in a dc microgrid . . . . . . . . . . . . . . . 100

4.2 Stability analysis for the geometric surfaces ............. 114

4.3 Simulation results showing the implementation of the digital control strategy for the dc microgrid . . . . . . . . . . . . . . 119 
4.4 Experimental results for the dc microgrid with reference geometric surfaces for step change in the loads . . . . . . . . . . . . . . . 123

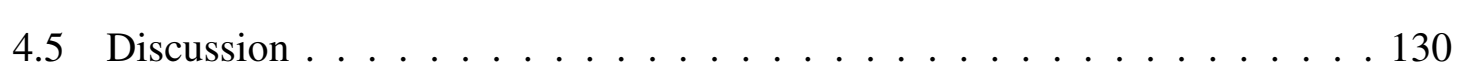

4.6 Conclusions . . . . . . . . . . . . . . . . . . . . . . . . . . .

5 Conclusions ........................ 133

5.1 Summary of accomplishments .................... 133

5.2 Recommendations for future work ......................... 135

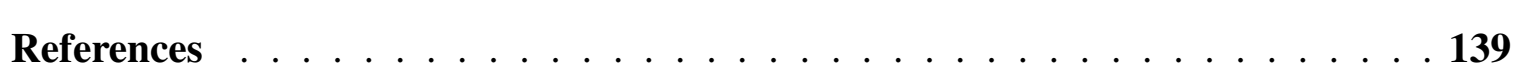

A MATLAB scripts and Simulink models . . . . . . . . . . . . . 157

A.1 MATLAB script for the solution of game-theoretic control BVP for the dc

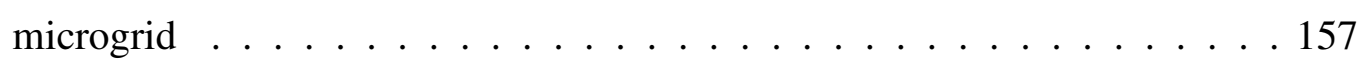

A.2 MATLAB script for the solution of optimal control BVP for the dc-dc boost

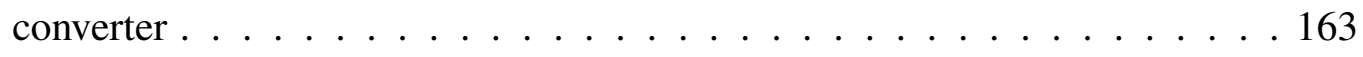

A.3 MATLAB script and Simulink model for the control of the dc-dc boost converter example system for a straight line reference surface . . . . . . . 165

A.4 MATLAB script and Simulink model for the control of the dc-dc boost converter example system for a sloped linear reference surface . . . . . . 168

A.5 MATLAB script and Simulink model for the control of the dc-dc boost converter example system for an optimal non-linear reference surface . . . . 171 
A.6 MATLAB script and Simulink model for the control of the dc microgrid for step change in the loads . . . . . . . . . . . . . . 175 


\section{List of Figures}

2.1 A general dc microgrid with $n$ source converters and $m$ POLCs considered for the derivation of the geometric manifolds. Adapted from Fig. 1 of [1]. . 18

2.2 DC Microgrid with 1 source converter and 2 POLCs. Adapted from Fig. 2 of $[1] \ldots \ldots \ldots \ldots \ldots \ldots \ldots$

2.3 POLC 2 stored energy time-domain simulations for three different cases illustrating the formation of the geometric manifolds in the dc microgrid. . . 29

2.4 POLC 2 input power time-domain simulations for three different cases illustrating the formation of the geometric manifolds in the dc microgrid. . . 30

2.5 Source converter geometric manifold in the energy-power domain for the example dc microgrid. . . . . . . . . . . . . . . . . . 30

2.6 POLC 1 geometric manifold in the energy-power domain for the example dc microgrid.

2.7 POLC 2 geometric manifold in the energy-power domain for the example de microgrid.

2.8 General de microgrid with $n$ source converters and $m$ POLCs in the voltage-current domain. Adapted from Fig. 8 of [1] . . . . . . . . . . . . 34 
2.9 Example de microgrid with '1' source converter and '2' POLCs in the voltage-current domain. Adapted from Fig. 9 of [1] . . . . . . . . . . . . 35

2.10 Source converter surface in the voltage-current domain for the example dc

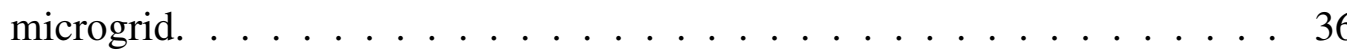

2.11 POLC 1 surface in the voltage-current domain for the example dc microgrid. 36

2.12 POLC 2 surface in the voltage-current domain for the example dc microgrid. 37

2.13 State Trajectories for the source converter for the example dc microgrid

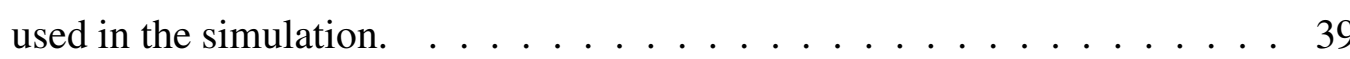

2.14 State Trajectories for the source converter for example dc microgrid used in an unknown situation. . . . . . . . . . . . . . 42

3.1 Digital memory lookup based controller block diagram for the dc-dc boost converter. Adapted from Fig. 13 of [1] . . . . . . . . . . . . . . . . 48

3.2 Circuit for the implementation of the digital sliding mode control strategy with two ADCs and a single memory lookup table in a dc-dc boost converter for resolution analysis.

3.3 State-plane plot for the digital sliding mode control strategy for a sloped reference surface showing the memory resolution effect for a resolution step size of 16. . . . . . . . . . . . . . . . 55

3.4 State-plane plot for the digital sliding mode control strategy for a sloped reference surface showing the memory resolution effect for a resolution step size of $32 \ldots \ldots \ldots \ldots \ldots \ldots \ldots$ 
3.5 State-plane plot for the digital sliding mode control strategy for a sloped reference surface showing the memory resolution effect for a resolution step size of $64 \ldots \ldots \ldots \ldots \ldots$

3.6 State-plane plot for the digital sliding mode control strategy for a sloped reference surface showing the memory resolution effect for a resolution step size of $128 \ldots \ldots \ldots \ldots \ldots$

3.7 Illustration of hysteresis generation due to time delay of a signal. . . . . . . 61

3.8 Dc-dc boost converter with resistive load.

3.9 Phase portrait of dc-dc boost converter states for the straight line reference sliding surface. . . . . . . . . . . . . . . . . 65

3.10 Time domain plot for the digital sliding mode control strategy for a straight line surface in a dc-dc boost converter. . . . . . . . . . . . . 66

3.11 State-plane plot for the digital sliding mode control strategy for a straight line surface in a dc-dc boost converter. . . . . . . . . . . 67 67

3.12 Experimental hardware set up for the digital hysteretic sliding mode control implementation in a dc-dc boost converter. . . . . . . . . . . 68

3.13 Experimental time domain plot for the digital sliding mode control strategy for a straight line surface in a dc-dc boost converter. . . . . . . . . . . 68

3.14 Experimental time domain plot in the steady state for the digital sliding mode control strategy for a straight line surface in a dc-dc boost converter. . 69 
3.15 ADC delay of $2.5 \mu \mathrm{s}$ generating a hysteresis band of $2.88 \mathrm{~A}$ for the digital sliding mode control strategy for a straight line surface in a dc-dc boost

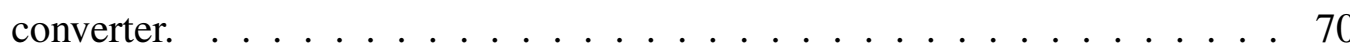

3.16 ADC delay of $2.2 \mu \mathrm{s}$ generating a hysteresis band of $1.88 \mathrm{~A}$ for the digital sliding mode control strategy for a straight line surface in a dc-dc boost converter. ...................... 70

3.17 ADC delay of $2.8 \mu \mathrm{s}$ generating a hysteresis band of $4.08 \mathrm{~A}$ for the digital sliding mode control strategy for a straight line surface in a dc-dc boost

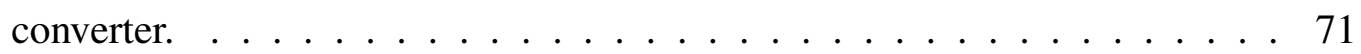

3.18 Sloped surface in the state-plane plot for the digital sliding mode control strategy in a dc-dc boost converter. . . . . . . . . . . . . 73

3.19 Phase portrait of dc-dc boost converter states for the sloped reference sliding surface. .......................... 76

3.20 Time domain plot for the digital sliding mode control strategy for a sloped reference surface in a dc-dc boost converter. . . . . . . . . . . 77

3.21 State-plane plot for the digital sliding mode control strategy for a sloped reference surface in a dc-dc boost converter. . . . . . . . . . 78

3.22 Experimental time domain plot for the digital sliding mode control strategy for a sloped reference surface in a dc-dc boost converter. . . . . . . . . 79

3.23 Experimental time domain plot in the steady state for the digital sliding mode control strategy for a sloped reference surface in a dc-dc boost converter. 80 
3.24 Experimental state-plane plot for the digital sliding mode control strategy for a sloped reference surface in a dc-dc boost converter. . . . . . . . . 80

3.25 Optimal surface in the state-plane plot for the digital sliding mode control strategy in a dc-dc boost converter. . . . . . . . . . . . . 82

3.26 Comparison of the original non-linear optimal surface with the approximated quadratic function in a state-plane plot for the optimal reference surface control in a dc-dc boost converter. . . . . . . . . . 87

3.27 Phase portrait of dc-dc boost converter states for the non-linear optimal reference sliding surface. . . . . . . . . . . . . . . . . 90

3.28 Time domain plot for the digital sliding mode control strategy for an optimal reference surface for a step change in load at $t=0.005 \mathrm{~s}$ in a dc-dc boost converter.

3.29 State-plane plot for the digital sliding mode control strategy for an optimal reference surface for a step change in load in a dc-dc boost converter. . . . .

3.30 Experimental time domain plot for the digital sliding mode control strategy for an optimal reference surface for a step change in load in a dc-dc boost

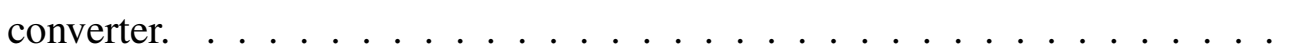

3.31 Experimental time domain plot in the steady state for the digital sliding mode control strategy for an optimal reference surface before the step change in load is applied in a dc-dc boost converter. . . . . . . . . . 93 
3.32 Experimental time domain plot in the steady state for the digital sliding mode control strategy for an optimal reference surface after the step change in load is applied in a dc-dc boost converter. . . . . . . . . . . 94

3.33 State-plane plot for the digital sliding mode control strategy for an optimal reference surface for a step change in load in a dc-dc boost converter with raw experimental data.

3.34 State-plane plot for the digital sliding mode control strategy for an optimal reference surface for a step change in load in a dc-dc boost converter with moving average experimental data. . . . . . . . . . . . .

4.1 Reference game-theoretic geometric surface for the source converter in the dc microgrid.

4.2 Reference game-theoretic geometric surface for the POLC 1 in the dc microgrid.

4.3 Reference game-theoretic geometric surface for the POLC 2 in the dc microgrid.

4.4 Comparison of the original surface for the source converter with the approximated piecewise quadratic functions in a state-plane plot. . . . . . 115

4.5 Comparison of the original surface for the POLCs with the approximated piecewise quadratic function in a state-plane plot. . . . . . . . . . . 115

4.6 Phase portrait of source converter states in the dc microgrid for the geometric reference sliding surface. 
4.7 Phase portrait of POLC 1 states in the dc microgrid for the geometric reference sliding surface. . . . . . . . . . . . . . . . . 119

4.8 Circuit diagram for the dc microgrid. . . . . . . . . . . . . . 120

4.9 Current and voltage profiles for the dc-dc boost converters in the dc microgrid for a step change in load at $t=0.1 s \ldots \ldots \ldots$. . . . . . . 121

4.10 State trajectories for the source converter in the dc microgrid for a step change in load.

4.11 State trajectories for POLC 1 in the dc microgrid for a step change in load. . 122

4.12 State trajectories for POLC 2 in the dc microgrid for a step change in load. . 122

4.13 Experimental set up for the digital sliding mode hysteretic controller implementation in a dc microgrid for a step change in load.

4.14 Experimental time domain plot for the source converter in the dc microgrid for a step change in load.

4.15 Experimental time domain plot for the source converter in the dc microgrid in the steady state before the step change in load is applied. . . . . . . . 126

4.16 Experimental time domain plot for the source converter in the dc microgrid in the steady state after the step change in load is applied.

4.17 State-plane plot for the source converter in the dc microgrid for a step change in load with raw experimental data. . . . . . . . . . . 127

4.18 State-plane plot for the source converter in the dc microgrid for a step change in load with moving average experimental data. . . . . . . . 128 
4.19 Experimental time domain plot for the POLCs in the dc microgrid for a step change in load. . . . . . . . . . . . . . . . . 128

4.20 Experimental time domain plot for the POLCs in the dc microgrid in the steady state before the step change in load is applied. . . . . . . . . . . . 129

4.21 Experimental time domain plot for the POLCs in the dc microgrid in the steady state after the step change in load is applied. . . . . . . . . . . 129

4.22 State-plane plot for the for the POLCs in the dc microgrid for a step change in load with raw experimental data. . . . . . . . . . . . . 130

4.23 Experimental state-plane plot for the for the POLCs in the dc microgrid for a step change in load with moving average experimental data. . . . . . . . 130

A.1 Simulink model for the dc-dc boost converter example system for a straight line reference surface. . . . . . . . . . . . . . . . . . . 167

A.2 Simulink model for the dc-dc boost converter example system for a sloped linear reference surface. . . . . . . . . . . . . . 171

A.3 Simulink model for the dc-dc converter example system for an optimal non-linear reference surface. . . . . . . . . . . . . . . 175

A.4 Simulink model for the dc microgrid for a step change in the loads. . . . . . 182 


\section{List of Tables}

2.1 System parameters for the solution of the game-theoretic control problem in the example dc microgrid . . . . . . . . . . . . . . . 29

2.2 Example dc microgrid initial parameters used in the simulation. . . . . . . 40

2.3 Example dc microgrid final parameters for a step change in load used in the simulation. .............................. 40

2.4 Example dc microgrid final parameters for a step change in source used in the simulation. ................................ 40

3.1 Example dc microgrid parameters for a sloped linear reference surface control.

3.2 Cost comparison between different digital controllers capable of implementing digital sliding mode control strategy in a dc-dc boost converter. 58

3.3 Example de microgrid parameters for a straight line surface control. . . . . 65

3.4 Initial system parameters for the optimal reference surface control in a dc-dc boost converter. . . . . . . . . . . . . . . . 85

3.5 Final system parameters for the optimal reference surface control in a dc-dc boost converter for step change in load. . . . . . . . . . . . . . . . 86 
4.1 Example dc microgrid initial parameters for step change in load used for simulation. . . . . . . . . . . . . . . . . . . 109

4.2 Example de microgrid final parameters for step change in loads used for simulation. . . . . . . . . . . . . . . . . . 110 


\section{Acknowledgments}

I would like to thank all the people who have made this dissertation possible. The first person I would like to thank is my advisor, Dr. Wayne W. Weaver, for his guidance, advice and encouragement. Without his patience and support, nothing would have been possible. I also want to thank the other members of my committee - Prof. Leonard J. Bohmann, Prof. Gordon G. Parker and Prof. Duane J. Bucheger, for their valuable feedback on my dissertation proposal. I would like to give a special thank you to Prof. Daniel R. Fuhrmann and Prof. Bruce A. Mork for giving me the opportunity to study at Michigan Tech. For their financial support, I would like to thank the National Science Foundation and the Department of Electrical and Computer Engineering, Michigan Tech.

There have been many others in the department who helped me a lot in every step of my graduate life. I would like to thank Chuck Sannes, Mark Sloat and Mike Chase for their tremendous support with the hardware work. My friend Ramachandra Kotecha deserves a very special thanks for helping me out with almost everything in the lab. I would also like to thank Trever J. Hassell and Arthur Jones for their help with my experiments. Michigan Tech's writing center deserves a very big thank you for helping me with my writing skills.

I would like to thank my father Kalyan Banerjee for his unconditional support and confidence in me. Finally, I want to thank my wife Aparajita for her love and support 
and for being the most important person in my life. Without her presence beside me, this would never have been possible. 


\section{Abstract}

In power electronic based microgrids, the computational requirements needed to implement an optimized online control strategy can be prohibitive. The work presented in this dissertation proposes a generalized method of derivation of geometric manifolds in a dc microgrid that is based on the a-priori computation of the optimal reactions and trajectories for classes of events in a dc microgrid. The proposed states are the stored energies in all the energy storage elements of the dc microgrid and power flowing into them. It is anticipated that calculating a large enough set of dissimilar transient scenarios will also span many scenarios not specifically used to develop the surface. These geometric manifolds will then be used as reference surfaces in any type of controller, such as a sliding mode hysteretic controller.

The presence of switched power converters in microgrids involve different control actions for different system events. The control of the switch states of the converters is essential for steady state and transient operations. A digital memory look-up based controller that uses a hysteretic sliding mode control strategy is an effective technique to generate the proper switch states for the converters.

An example dc microgrid with three dc-dc boost converters and resistive loads is considered for this work. The geometric manifolds are successfully generated for transient events, 
such as step changes in the loads and the sources. The surfaces corresponding to a specific case of step change in the loads are then used as reference surfaces in an EEPROM for experimentally validating the control strategy. The required switch states corresponding to this specific transient scenario are programmed in the EEPROM as a memory table. This controls the switching of the dc-dc boost converters and drives the system states to the reference manifold. In this work, it is shown that this strategy effectively controls the system for a transient condition such as step changes in the loads for the example case. 


\section{Chapter 1}

\section{Introduction and Background}

\subsection{Motivation}

In modern power systems there is a growing concern about energy and the environment [2]. The smart grid and microgrid has been proposed as a standard of the future grid with efficient access to renewable resources and distributed generations [3, 4]. The use of game-theory in multi-player systems can be an effective optimization technique to control the operations in a smart grid and a microgrid [5, 6]. Game theory is a branch of mathematics that is a study of the phenomena observed when decision makers (called players) interact [7]. The basic assumption of game theory is that the players are rational and they reason strategically. Each player in the game has its own objective. A strategic 
interaction between all the players based on the desired objective of each player result in an outcome that may or may not have been intended by the players. This outcome is the solution of the game. The work in [8] summarizes different applications of game theory in smart grid. A distributed energy management system has been developed based on game theory in [9]. Non-cooperative game theory can be used for load balancing, as discussed in [10]. P. Aristidou et al. discussed in [11] how a cooperative game theoretic approach has been used in a microgrid model. A game-theoretic algorithm to predict the energy needs based on price is used in [12]. A game-theoretic approach to investigate storage in a multi-player smart grid is discussed in [13]. A distributed load management scheme is discussed based on a game-theoretic point of view in [14]. Therefore, game-theory is an extremely useful optimization tool in multi-player systems.

Point of load converters (POLC) in microgrids are highly efficient controllable power electronic elements that translate energy to and from the power network [15]. A common choice of state variables in the model of a POLC is the inductor current and the capacitor voltage [16]. However, with an energy balance model of the POLC, the modeling can be simplified [17]. Previous work related to the modeling of dc-dc converters in a power network has been done in the energy-impedance domain [18] and the energy-conductance domain [19]. These energy balance models are based on the fact that all types of converters have some form of energy storage devices that are capable of supplying and absorbing power [20]. These models of dc-dc converters in the energy-impedance or energy-conductance domain have been done keeping in mind that a power network uses bus 
admittance or bus conductance matrices and making a conductance or admittance system state is beneficial [21]. The selection of the states need to be made even more general by considering the power flowing into different sections of the power network and the stored energies in the converters to be the system states [1]. Conversion to other states as per the requirement of the system design is possible from these power and energy states.

In modern day smart grids and microgrids, the usage of dc-dc power electronic converters have increased due to the requirement of various levels of dc voltages in home and office facilities [22]. Switching in the power electronics is a non-linear phenomena and application of the traditional linear control techniques are often not suitable [23]. The classical linear control technique has limitations in large signal transients such as step changes in the load or startup processes [24]. Thus, there is a need for a control technique which is capable of dealing with non linearities and wide variations in load, while ensuring uninterrupted operation and at the same time provide fast transient response [25]. This advanced capability often comes at the price of a complex and costly control hardware [26]. Switching converters form a variable structure system and thus sliding mode control is an effective and simpler technique [27] than other robust control schemes which are computationally intensive [28, 29]. Previous work has been done on the development of sliding mode control strategy in a power converter, which drives the system states to some reference surface [30, 31, 32]. In microgrids, the use of digital control in switching power converters has increased due to the decreased cost of digital ICs [33]. Some work has been done with the digital implementation of a hysteretic sliding mode control strategy 
by implementing a predetermined surface inside the digital controller [34]. There lies a need to implement a fast, nonlinear and optimal control strategy in low cost and reliable hardware that will control the switching of power electronic converters in a multi-agent system, such as a microgrid. The interactions between all the energy storage devices in the microgrid can be treated as a game where the energy storage devices are the players having their own objective to achieve. During a transient event, it is possible that the objectives of all the players may not be met due to physical constraints of the system. A game-theoretic control problem is based on the interactions between the players and takes into account their individual objectives, to give an outcome that conforms to the physical constraints of the system. Therefore, a game-theoretic control is needed for the microgrid. This dissertation will derive a game-theoretic geometric manifold in a dc microgrid for various transient events and implement a digital sliding mode hysteretic control technique that will drive the state trajectories to this geometric manifold during the transient conditions.

\subsection{Microgrids}

The technology behind electricity generation, transmission and distribution is constantly changing [35, 36]. This is due to the advancement of technology and various economic and environmental incentives [37]. A centralized generation strategy is gradually giving its way to distributed generation [38, 39, 40]. The main drawbacks for a centralized generation are high transmission and distribution costs, challenges in rural electrification, 
absence of any back up generation and environmental reasons [41]. These drawbacks proved to be the driving forces for the revival of distributed generation. They are gradually becoming realible sources of generation owing to its back up facility and conforming to the environmental requirements [42]. The emerging technologies in distributed generation, such as microturbines, photovoltaics, fuel cells, etc. have lower cost and emissions [43, 44, 45, 46]. The potential of these emerging distributed generation technologies can be realized by viewing the generation and the loads as a subsystem defined as a microgrid [47]. The microgrid integrates distributed generations without causing any disruption in the power system. The microgrid also allows for local control of the distributed generation units [48] and attests to the flexibility to operate autonomously during any disturbances in the main network [49].

Most microgrids have an ac distribution structure, which requires the use of inverters for dc sources, such as photovoltaics and fuel cells [50]. This decreases the conversion efficiency. Also greater number of inverters in the system means greater cost [51]. For a dc microgrid, the system efficiency increases because of the reduced conversion losses owing to the absence of an inverter in the system [52, 53]. Also, dc microgrids do not require any synchronization [54]. Usage of power electronics in microgrids enable the capability of using the stored energy, even bus capacitance, to mitigate system transients [1]. 


\subsection{Game-theoretic control}

Game theory is a branch of mathematics that deals with multiple decision makers, called players, that interact in a common system [55]. When a system is influenced by only one player, an optimal condition, based on that player's decision, is achieved. This is the basis of an optimal control problem [56]. However, when a number of players in the system interact and make decisions, the choice of one player will influence the decisions made by all others. So the concept of optimality is modified for multi-player games. In game theory, a special form of optimality, called the Nash Equilibrium, is used. Nash Equilibrium is a solution to a game where one player cannot improve its outcome by altering its decision unilaterally [57]. Z. M. Fadlullah et al. gave a detailed illustration and mathematical formulation of a game in [8].

The decisions made by a player in a game may or may not be based on any communication between all the players. Non-cooperative game is one in which players make decisions independently [58]. Thus, while players could cooperate, any cooperation must be self-imposed. When there is communication between the players and the decision of a player is based on the knowledge of other players' decisions, the game is called a cooperative game [59]. In microgrids and power systems in general, there are interconnected loads and sources that have their own objectives to achieve in the system. This gives rise to time dependent states in the system that are part of the game. This type 
of a game is called a differential game [60].

Game-theoretic control [61, 62, 63] is a new approach for distributed resource allocation [64, 65]. A game-theoretic control is an approach where interaction of distributed players are studied where each player has its own objective to achieve. Here, each player makes local decisions based on local information available. The solution to the game-theoretic control is a global decision from a combination of all of the local decisions made by each player [66]. A global decision means the outcome of the game based on the individual objectives of all the players and the system constraints.

Application of game-theoretic control requires identifying the players in the game, specifying their objectives and controlling what information should reach them such that the desired global decision outcome is acceptable within the required design limits. Application of this game-theoretic control approach is widely applicable to many engineering fields, such as wireless and sensor network applications, distributed energy resource control in power systems, etc. A comprehensive list of applications may be found in [62]. 


\subsection{Sliding mode control}

Sliding mode control technique is an effective technique in variable structure systems, such as power electronic converters [67]. This control scheme involves the selection of a switching manifold, such that the system trajectories exhibit desirable behavior when confined to this manifold, wherein the dynamics of any nonlinear system is altered via application of a high-frequency switching control [68]. A pure sliding condition means infinite switching frequency [69]. In real time hardware that is not feasible and desirable. Therefore, the switching should occurs between two threshold values [70] around the sliding surface. This is also known as hysteretic sliding mode control technique [71]. The switch states for each of the power converters for this work has to be determined by the sliding control law

$$
s=f(x)=0
$$

where $x$ represents the converter states and $f(x)$ is the deviation of the states from the derived geometric surface [1]. The switching control law is therefore given by [72, 73]

$$
q= \begin{cases}0 & \text { if } s-\frac{h}{2}>0 \\ 1 & \text { if } s+\frac{h}{2}<0\end{cases}
$$


where $q$ is the switch state of the converter and $h$ is the hysteretic band used to lower the switching frequency of the switch around the surfaces [18]. In nonlinear systems, Lyapunov's direct method is one of the ways to find out whether the system is stable without actually solving the dynamic equations for the system. This method states that if there exists a continuous positive definite function $\mathrm{V}(\mathrm{x})$ for a system, such that $V(x)$ is a negative semi definite function, then the system is said to be stable in the sense of Lyapunov [74]. This is a sufficient condition to show the stability of a system. If a positive definite Lyapunov candidate function

$$
V(x)=\frac{1}{2} s^{2}
$$

is chosen, for stability,

$$
V(x)<0
$$

Substituting (1.3) in (1.4), the required condition for stability is

$$
s \dot{s}<0 .
$$

If (1.5) is satisfied for a region of the state space that contains the sliding surface (1.1), then the state trajectories that leave any point on this region will converge to the sliding surface and once they reach it, they will stay on it [75]. A hysteretic sliding mode control 
technique is considered to be an extremely robust technique because the entire system dynamics is governed by the sliding surface, not the system parameters [76]. Switching in power electronic converters form a variable structure system, and therefore a power electronic converter is an ideal candidate for the application of this hysteretic sliding mode control technique [77].

\subsection{Digital control in power electronics}

Digital control methods and digital controllers, such as microcontrollers, digital signal processors (DSP) and programmable logic devices (PLC), have become an integral part in the control of switching power devices [78, 79, 80]. The use of digital control in switching power converters has increased due to the decreased cost of integrated chips [33]. Power electronic devices are operated at relatively high switching frequencies (hunderds of $\mathrm{kHz}$. to $\mathrm{MHz}$. range) to reduce the size and weight of passive energy storage components, and to enable fast dynamic regulation [81]. Hence, a fast digital processor becomes necessary. These challenges require new solutions and this has resulted in the introduction of Electrically Erasable Programmable Read-Only Memory chips (EEPROM) in the control of power electronics. An EEPROM, such as AT28C256, can act as a memory lookup table inside the controller [82]. It can read in the system states and lookup pre-programmed switch states based on the system states. At low power levels, upto a few kilowatts, the use

of EEPROMs contribute to simpler design and lower cost as compared to microcontrollers 
and other programmable devices.

Analog to digital converters (ADC) are used to convert analog signals to digital [83]. These ADCs have an inherent delay in the signal processing time [84]. This delay generates the required hysteresis for the sliding-mode controller. This simplifies the hardware complications by reducing the number of bits necessary in the EEPROM. There is no need to use additional memory locations for hysteresis generation. The ADC generates the required hysteresis and the sliding law is stored in the EEPROM. This will form a digital sliding mode hysteretic controller that will be cheap, fast and robust at the same time.

\subsection{Dissertation organization}

This dissertation deals with the development of a digital hysteretic sliding mode control strategy in a switching converter in a dc microgrid where the reference manifold is derived a-priori based on a game-theoretic approach. The purpose of the game-theoretic control is to derive a family of state trajectories corresponding to many transient events and generate a complete geometric manifold for each switching converter in the dc microgrid. The purpose of the digital sliding mode hysteretic controller is to drive the system states to these geometric manifolds. The control strategy will be tested for a single dc-dc boost converter as well as a dc microgrid comprising of multiple dc-dc boost converters. The strategy, 
though tested for dc-dc boost converters, will be equally applicable for other converter topologies.

Chapter 2 will introduce a generalized method to formulate a game-theoretic control problem in a dc microgrid with $m+n$ power electronic converters and to solve the problem for an example system with three power electronic converters. Here, $m$ is the number of power electronic converters connected to the sources and $n$ is the number of POLCs in the microgrid. The outcome of this chapter will be a guideline to derive geometric manifolds for any number of converters in a microgrid for any transient event in the system. In [1], it has been shown that all the energy storage devices in a microgrid can be treated as different players in the system. Each player has its own objective to achieve in this game, such as keeping their terminal voltages constant, and their combined objectives are met for the different transient events, such as a step change in the load or the source. This is a non-cooperative game-theoretic control problem, where the players in the game make their decisions based only on some local information available to them. When many such transient events are solved, all the solutions form a geometric surface that is used for real-time implementation in dc microgrids [1].

Chapter 3 will show a method of implementing a digital sliding mode hysteretic control technique in a switching power electronic converter. A dc-dc boost converter will be chosen to validate the strategy. The stability analysis of various reference surfaces chosen for the controller will be performed in this chapter. The reference surfaces to be chosen will be 
both linear and non-linear surfaces in the state-plane diagram [85]. The work shown in this chapter will use [34] as the starting point and will design and implement a digital sliding mode hysteretic control method in a dc-dc boost converter where the sliding surfaces will be predetermined and stored in a low-cost memory circuit, instead of using any form of software component, such as a DSP, in the design. It will also be shown that by using ADCs in the process, the control can be improved by eliminating the need to derive and implement a hysteresis band which limits the effective switching frequency [84]. Since the sliding surfaces will be derived a-priori, the system response time will be reduced.

The work shown in Chapter 4 will be an extension of the work shown in Chapter 3 , Here, the digital sliding mode hysteretic control method will be implemented in a dc microgrid with three dc-dc boost converters, i.e. a larger system will be considered for the real-time implementation of the controller. The sliding surfaces will be derived a-priori based on a model based game-theoretic trajectories solutions of the converters as shown in Chapter 2 , The sliding surfaces will be part of the generalized manifolds derived in Chapter 2. The stability analysis for these surfaces will also be done for this example system.

Chapter 5 summarizes the primary achievements of this work by discussing the future areas of research that may be explored based on the outcome of this work. This dissertation develops a general guideline for designing and solving game-theoretic control problems in a microgrid and it outlined a method of real-time implementation of a non-linear robust control technique in a microgrid that uses the solution of the game-theoretic control 
problem. However, many areas still remain to be explored, such as considering the effects of transient conditions other than steps in sources and loads. 


\section{Chapter 2}

\section{Game-theoretic geometric manifolds in}

\section{de microgrids}

In [1], it was shown that all the energy storage devices in a microgrid can be treated as players in the system. Each player has its own objectives and their combined objectives are met for transient events, such as a step change in the load or the source. When many such transient events are solved, all the solutions combine together to form a geometric manifold that is used for real-time implementation in dc microgrids. 1

\footnotetext{
${ }^{1}$ The method of derivation of the geometric manifolds in Chapter2] is adapted from [1] @2012, IEEE. Some texts pertaining to the explanation of the method and the system are adapted from [1] (02012, IEEE. The generalized system, example system and controller block diagrams have also been modified from [1] @2012, IEEE.
} 


\subsection{Generalized game-theoretic control manifolds in a dc microgrid}

In a dc microgrid, different system components may have different objectives to achieve. Also, there may be many different transient situations taking place in the system at different instants. Derivation of the geometric manifolds in a dc microgrid therefore requires the formulation of the problem with defined objectives and transient scenarios. Then the solution to the problem for all the transient events will generate the required geometric manifolds in the dc microgrid.

\subsubsection{Derivation of the game-theoretic control manifold}

The formulation of the control problem is discussed in Section 2.1.1.1 and the method of solution to the problem is shown in Section 2.1.1.2.

\subsubsection{Formulation of the game-theoretic control problem}

Consider a general microgrid with $n$ dc-dc boost converters in the set $\mathscr{N}$ of energy sources and $m \mathrm{dc}-\mathrm{dc}$ boost converters in the set $\mathscr{M}$ of POLCs. The source converters have resistive 
loads $R_{s i} \forall i \in \mathscr{N}$. The output of the POLCs are connected to resistive loads $R_{j} \forall j \in \mathscr{M}$. A block diagram of the generalized system is shown in Fig. 2.1][1]. The POLCs in Fig. 2.1 are responsible for supplying power from a power network to their final loads. Any set of independent variables may be chosen as the state variables [16]. In this work, the power flowing into the converter and the energy stored in the converter are used as the states [1]. All converters have some form of energy storage devices that are capable of supplying and absorbing power [20]. Thus, the sum of power in a POLC is given as

$$
p_{\text {in }}-p_{\text {out }}-p_{\text {stored }}-p_{\text {loss }}=0
$$

The time rate of change of energy is power. Then neglecting losses, (2.1) can be expressed as

$$
\dot{w}=p_{\text {in }}-p_{\text {out }}
$$

where $\dot{w}$ is the power stored. For dc-dc power electronic converters, the energy state $w$ can be expressed as

$$
w=\frac{1}{2} C v_{\text {out }}^{2}
$$

where $\mathrm{C}$ is the capacitance of the output capacitor of the power electronic converter and $v_{\text {out }}$ is the voltage across it. The inductors in the converter circuits primarily act as energy 


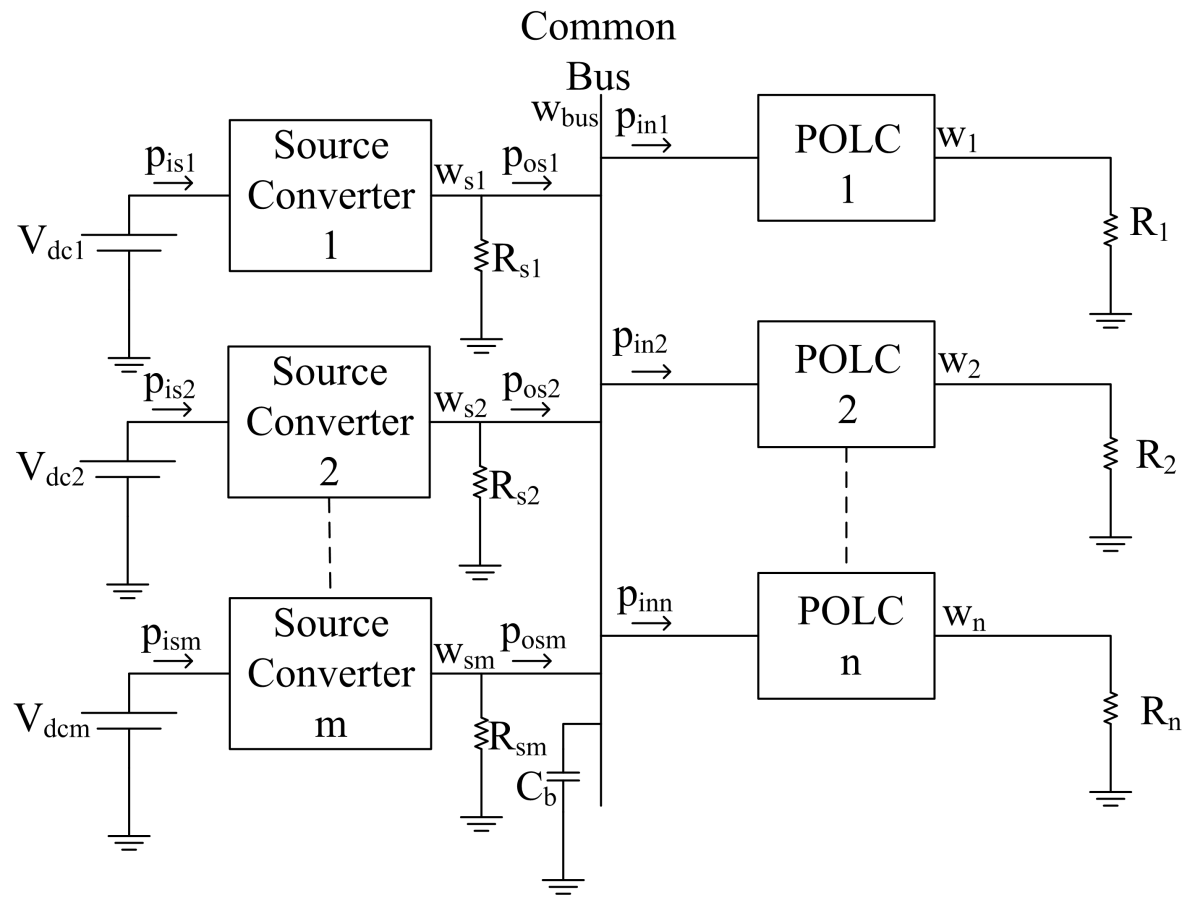

Figure 2.1: A general dc microgrid with $n$ source converters and $m$ POLCs considered for the derivation of the geometric manifolds. Adapted from Fig. 1 of [1].

transfer components, while the true energy storage is in the electric field of the capacitors. Therefore, only the capacitor energies are considered [19].

Note that (2.2) is true not only for a POLC, but is also true for any energy storage device in a power network. Therefore, in Fig. 2.1, the source converters may also be treated as energy storage devices and the energy stored in them may be considered to be a suitable state variable. The common bus in Fig. 2.1 has a capacitor and thus can also be treated as an energy storage element in the microgrid. In addition to the energy storage, the rates of change of the input and output power flows may be considered to have a general state 
dependency of the form

$$
\dot{p}=u
$$

where $u$ is the control input. The input power

$$
p=V_{\text {in }} i_{\text {in }}
$$

where $V_{\text {in }}$ is the input voltage and $i_{\text {in }}$ is the input current to the converter. Therefore, for a dc-dc boost converter, (2.4) reduces to

$$
\dot{p}=V_{\text {in }} \dot{i}_{\text {in }}=V_{\text {in }}\left[\frac{V_{\text {in }}-(1-q) v_{\text {out }}}{L}\right]
$$

where $q$ is the switch state of the dc-dc boost converter. Hence, using (2.6) in (2.4) gives

$$
u=V_{\text {in }}\left[\frac{V_{\text {in }}-(1-q) v_{\text {out }}}{L}\right]
$$

Therefore, the control input $u$ can be transformed into direct switch states of a converter. Thus, in the microgrid model from Fig 2.1, each converter has state variables of stored energy and input power. The rate of change of the power flows in the different sections of the network are the control inputs. In a compact form, the state variables may be written as $w_{s i}, w_{b u s}, w_{j}, p_{i s i}, p_{o s i}$ and $p_{i n j}, \forall i \in \mathscr{N}$ and $\forall j \in \mathscr{M}$. The rate of change of power flowing into the source converters, the rate of change of power flowing into the common bus and 
the rate of change of power flowing into the POLCs are the controls for the network. Thus the dimension of this system is $3 n+1+2 m[1]$.

For the system shown in Fig. 2.1, during a transient condition, such as a step change in the load or the source, the objective of the control is to conserve the energy stored in the converters and the common bus. The set of dynamic equations for the system of Fig 2.1 is given as

$$
\begin{aligned}
& \dot{w}_{s i}=p_{i s i}-\frac{2 w_{s i}}{C_{s i} R_{s i}}-p_{o s i} \\
& \dot{w}_{b u s}=\sum_{i=1}^{n} p_{o s i}-\sum_{j=1}^{m} p_{\text {inj }} \\
& \dot{w}_{j}=p_{\text {inj }}-\frac{2 w_{j}}{C_{j} R_{j}} \\
& \dot{p}_{i s i}=u_{1 i} \\
& \dot{p}_{\text {osi }}=u_{2 i} \\
& \dot{p}_{\text {inj }}=u_{3 j} .
\end{aligned}
$$

for all $i \in \mathscr{N}$ and $j, k \in \mathscr{M}$. The rate of change of the power flow in (2.8) are the controls $u_{1 i}, u_{2 i}$ and $u_{3 j}$

To derive the game-theoretic optimal state trajectories of the system, an objective function of the general form

$$
J=\int_{0}^{t_{f}} g(x) d t
$$


is chosen [86]. The choice of the path objective for all the players is

$$
\begin{aligned}
g(x)=\sum_{i=1}^{n} & \left(w_{\text {si }}-w_{\text {sinom }}\right)^{2}+\left(w_{\text {bus }}-w_{\text {busnom }}\right)^{2} \\
& +\sum_{j=1}^{m}\left(w_{j}-w_{\text {jnom }}\right)^{2}+\sum_{i=1}^{n} u_{1 i}{ }^{2} \\
& +\sum_{i=1}^{n} u_{2 i}{ }^{2}+\sum_{j=1}^{m} u_{3 j}{ }^{2}
\end{aligned}
$$

For all expressions in (2.10), $i \in \mathscr{N}$ and $j, k \in \mathscr{M}$. Also, $w_{\text {sinom }}, w_{\text {busnom }}$ and $w_{\text {jnom }}$ are the nominal values of the respective energy functions. The path objective (2.10) seeks to keep the total energy as close to nominal values as possible and the rate of change of the power flow functions are minimized. The power flow functions ensure that they are at least continuous from their nominal values, i.e. they should not change rapidly with time. It should be noted that in the following examples, equal weight is given to all the components of the objectives. If the weights are not equal, the objective of the player with greater weight will get higher priority. Each energy storage device in the system is a player having a defined objective. In (2.10), the term $\sum_{i=1}^{n}\left(w_{s i}-w_{\text {sinom }}\right)^{2}$ is the sum of the objectives of all the source converters. The objective of each source converter is to maintain its energy as close to the nominal value as possible. The term $\left(w_{\text {bus }}-w_{\text {busnom }}\right)^{2}$ is the objective of the common bus and $\sum_{j=1}^{m}\left(w_{j}-w_{j n o m}\right)^{2}$ is the sum of the objectives of all the POLCs. The combined objectives of all the players form the objective function $(2.10)$. During a transient condition the objective of the game-theoretic control is to make the state trajectories travel from the initial operating conditions to the new steady-state conditions 
based on the minimization of the objective function (2.9). This problem is called a game because multiple players with different objectives are involved. The solution to this game should ensure that the players will not have the motivation to change the outcome. This will be the Nash equilibrium. The initial conditions for the system are

$$
\begin{array}{cl}
w_{\text {si }}(0)=w_{\text {sinom }} & p_{\text {isi }}(0)=p_{\text {isio }} \\
w_{\text {bus }}(0)=w_{\text {busnom }} & p_{\text {osi }}(0)=p_{\text {osio }} \\
w_{j}(0)=w_{\text {jnom }} & p_{\text {inj }}(0)=p_{\text {injo }} .
\end{array}
$$

To simplify the objectives around the nominal operating points, (2.10) is modified to

$$
\begin{gathered}
g(x(t))=\sum_{i=1}^{n}\left(\Delta w_{s i}\right)^{2}+\left(\Delta w_{\text {bus }}\right)^{2}+\sum_{j=1}^{m}\left(\Delta w_{j}\right)^{2} \\
+\sum_{i=1}^{n} u_{1 i}{ }^{2}+\sum_{i=1}^{n} u_{2 i}{ }^{2}+\sum_{j=1}^{m} u_{3 j}{ }^{2}
\end{gathered}
$$

where

$$
\begin{aligned}
& \Delta w_{s i}=\left(w_{\text {si }}-w_{\text {sinom }}\right) \\
& \Delta w_{\text {bus }}=\left(w_{\text {bus }}-w_{\text {busnom }}\right) \\
& \Delta w_{j}=\left(w_{j}-w_{\text {jnom }}\right) .
\end{aligned}
$$


The state equations for the system are

$$
\begin{aligned}
\dot{\Delta w_{s i}}=p_{i s i}-\frac{2\left(\Delta w_{s i}+w_{\text {sinom }}\right)}{C_{s i} R_{s i}}-p_{o s i} & \dot{p}_{i s i}=u_{1 i} \\
\dot{\Delta w_{b u s}}=\sum_{i=1}^{n} p_{o s i}-\sum_{j=1}^{m} p_{i n j} & \dot{p}_{o s i}=u_{2 i} \\
\dot{\Delta w_{j}}=p_{i n j}-\frac{2\left(\Delta w_{j}+w_{j n o m}\right)}{C_{j} R_{j}} & \dot{p}_{i n j}=u_{3 j} .
\end{aligned}
$$

The initial conditions are

$$
\begin{aligned}
\Delta w_{s i}(0)=0 & p_{\text {isi }}(0)=p_{\text {isio }} \\
\Delta w_{\text {bus }}(0)=0 & p_{\text {osi }}(0)=p_{\text {osio }} \\
\Delta w_{j}(0)=0 & p_{\text {inj }}(0)=p_{\text {injo }} .
\end{aligned}
$$

\subsubsection{Solution to the game-theoretic control problem}

The problem consists of the state equations (2.14), the objective function (2.9) and the initial conditions (2.15). For the solution of the game-theoretic control problem, Pontryagin's minimum principle [18, 86] is used. However, the minimum principle is capable of providing only open loop solutions [86]. The open loop solutions of various a-priori events will be used to form a control manifold as the basis of a real-time feedback 
control algorithm. The Hamiltonian for the path minimization is

$$
\begin{aligned}
H=\sum_{i=1}^{n} & \left(\Delta w_{s i}\right)^{2}+\left(\Delta w_{\text {bus }}\right)^{2}+\sum_{j=1}^{m}\left(\Delta w_{j}\right)^{2} \\
& +\sum_{i=1}^{n} u_{1 i}{ }^{2}+\sum_{i=1}^{n} u_{2 i}{ }^{2}+\sum_{j=1}^{m} u_{3 j}{ }^{2} \\
& +\lambda_{1 i}\left(p_{i s i}-\frac{2\left(\Delta w_{s i}+w_{\text {sinom }}\right)}{C_{s i} R_{\text {si }}}-p_{\text {osi }}\right) \\
& +\lambda_{2}\left(\sum_{i=1}^{n} p_{\text {osi }}-\sum_{j=1}^{m} p_{\text {inj }}\right) \\
& +\lambda_{3 j}\left(p_{\text {inj }}-\frac{2\left(\Delta w_{j}+w_{j n o m}\right)}{C_{j} R_{j}}\right) \\
& +\lambda_{4 i} u_{1 i}+\lambda_{5 i} u_{2 i}+\lambda_{6 j} u_{3 j}
\end{aligned}
$$

where $\lambda_{1 i}, \lambda_{2}, \lambda_{3 j}, \lambda_{4 i}, \lambda_{5 i}$ and $\lambda_{6 j}$ are the co-states [86]. The solution for the controls $u_{1 i}{ }^{*}$, $u_{2 i}{ }^{*}$ and $u_{3 j}{ }^{*}$ are found from

$$
\begin{aligned}
& \frac{d H}{d u_{1 i}}=0 \\
& \frac{d H}{d u_{2 i}}=0 \\
& \frac{d H}{d u_{3 j}}=0
\end{aligned}
$$

and the co-states are found from

$$
\begin{array}{ll}
\dot{\lambda}_{1 i}=-\frac{d H}{d\left(\Delta w_{s i}\right)} & \dot{\lambda}_{4 i}=-\frac{d H}{d\left(p_{i s i}\right)} \\
\dot{\lambda}_{2}=-\frac{d H}{d\left(\Delta w_{\text {bus }}\right)} & \dot{\lambda}_{5 i}=-\frac{d H}{d\left(p_{\text {osi }}\right)} \\
\dot{\lambda}_{3 j}=-\frac{d H}{d\left(\Delta w_{j}\right)} & \dot{\lambda}_{6 j}==-\frac{d H}{d\left(p_{\text {inj }}\right)} .
\end{array}
$$


Thus the augmented set of equations are

$$
\begin{aligned}
\dot{\Delta w_{s i}}=p_{i s i}-\frac{2\left(\Delta w_{s i}+w_{\text {sinom }}\right)}{C_{s i} R_{s i}}-p_{o s i} & \dot{\lambda}_{1 i}=-\frac{d H}{d\left(\Delta w_{s i}\right)} \\
\dot{\Delta w_{b u s}}=\sum_{i=1}^{n} p_{o s i}-\sum_{j=1}^{m} p_{i n j} & \dot{\lambda}_{2}=-\frac{d H}{d\left(\Delta w_{b u s}\right)} \\
\dot{\Delta w_{j}}=p_{\text {inj }}-\frac{2\left(\Delta w_{j}+w_{\text {jnom }}\right)}{C_{j} R_{j}} & \dot{\lambda}_{3 j}=-\frac{d H}{d\left(\Delta w_{j}\right)} \\
\dot{p}_{i s i}=u_{1 i}^{*} & \dot{\lambda}_{4 i}=-\frac{d H}{d\left(p_{i s i}\right)} \\
\dot{p}_{o s i}=u_{2 i}{ }^{*} & \dot{\lambda}_{5 i}=-\frac{d H}{d\left(p_{o s i}\right)} \\
\dot{p}_{\text {inj }}=u_{3 j}{ }^{*} & \dot{\lambda}_{6 j}=-\frac{d H}{d\left(p_{\text {inj }}\right)} .
\end{aligned}
$$

The boundary conditions are

$$
\begin{aligned}
\Delta w_{\text {si }}(0) & =0 & \lambda_{1 i}\left(t_{f}\right) & =0 \\
\Delta w_{\text {bus }}(0) & =0 & \lambda_{2}\left(t_{f}\right) & =0 \\
\Delta w_{j}(0) & =0 & \lambda_{3 j}\left(t_{f}\right) & =0 \\
p_{\text {isi }}(0) & =p_{\text {isio }} & \lambda_{4 i}\left(t_{f}\right) & =0 \\
p_{\text {osi }}(0) & =p_{\text {osio }} & \lambda_{5 i}\left(t_{f}\right) & =0 \\
p_{\text {inj }}(0) & =p_{\text {injo }} & \lambda_{6 j}\left(t_{f}\right) & =0 .
\end{aligned}
$$

The boundary conditions imply that the co-states, which are actually variable cost functions, should reach a value of zero after time $t_{f}$, thereby minimizing the overall objective function. This is a two-point boundary value problem (BVP). For an optimal solution, the Hamiltonian is a minimized scalar value. The solution of (2.19) with the boundary conditions (2.20) gives the trajectories for the stored energies $w_{s i}, w_{b u s}$ and $w_{j}$ 


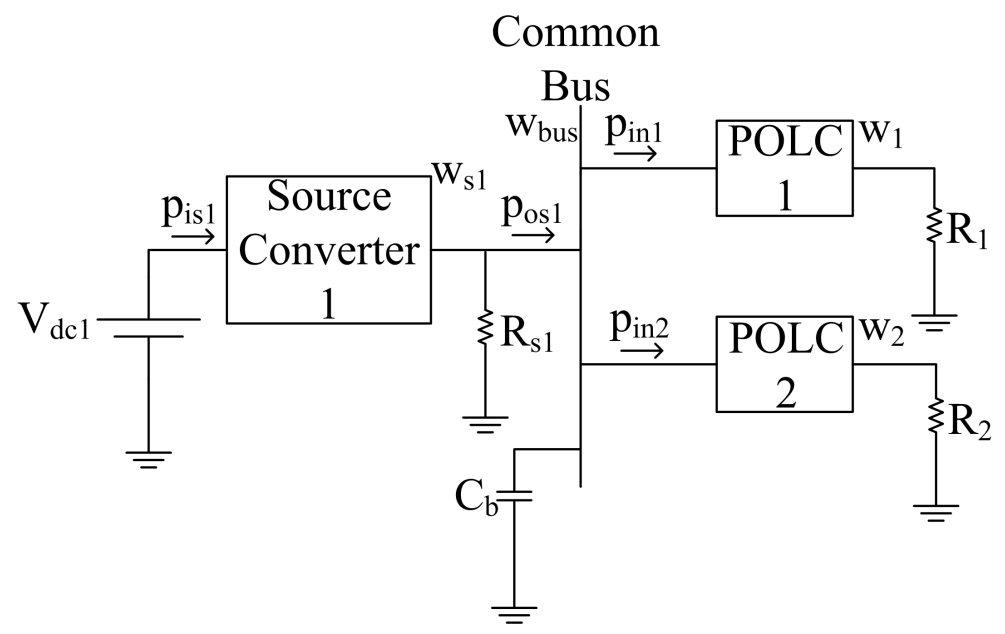

Figure 2.2: DC Microgrid with 1 source converter and 2 POLCs. Adapted from Fig. 2 of [1].

and the power flows $p_{i s i}, p_{o s i}$ and $p_{i n j}$ for transient conditions, such as a step change in the load or the source. This solution gives optimal operating points of all the players in the system. Not necessarily they are the individual nominal operating points for each of them, but an operating point based on the influence of the other players' objectives in the game.

An example system consisting of one source converter and two POLCs is considered with $n=1$ and $m=2$ as shown in Fig 2.2. With $n=1$ and $m=2$ in (2.16) through (2.20), the 
augmented set of equations for the example system of Fig. 2.2. is

$$
\begin{array}{ll}
\dot{\Delta w_{s 1}}=p_{i s 1}-\frac{2\left(\Delta w_{s 1}+w_{s 1 n o m}\right)}{C_{s 1} R_{s 1}}-p_{o s 1} \dot{\lambda}_{11}=\frac{2 \lambda_{11}}{C_{s 1} R_{s 1}}-2 \Delta w_{s 1} \\
\dot{\Delta w_{\text {bus }}}=p_{\text {os } 1}-p_{\text {in } 1}-p_{\text {in } 2} & \dot{\lambda}_{2}=-2 \Delta w_{\text {bus }} \\
\dot{\Delta w_{1}}=p_{\text {in } 1}-\frac{2\left(\Delta w_{1}+w_{1 n o m}\right)}{C_{1} R_{1}} & \dot{\lambda}_{31}=\frac{2 \lambda_{31}}{C_{1} R_{1}}-2 \Delta w_{1} \\
\dot{\Delta w_{2}}=p_{\text {in } 2}-\frac{2\left(\Delta w_{2}+w_{2 n o m}\right)}{C_{2} R_{2}} & \dot{\lambda}_{32}=\frac{2 \lambda_{32}}{C_{2} R_{2}}-2 \Delta w_{2} \\
\dot{p}_{\text {is } 1}=-\frac{\lambda_{41}}{2} & \dot{\lambda}_{41}=-\lambda_{11} \\
\dot{p}_{\text {os } 1}=-\frac{\lambda_{51}}{2} & \dot{\lambda}_{51}=-\lambda_{2}+\lambda_{11} \\
\dot{p}_{\text {in } 1}=-\frac{\lambda_{61}}{2} & \dot{\lambda}_{61}=\lambda_{2}-\lambda_{31} \\
\dot{p}_{\text {in } 2}=-\frac{\lambda_{62}}{2} & \dot{\lambda}_{62}=\lambda_{2}-\lambda_{32}
\end{array}
$$

and the boundary conditions are

$$
\begin{array}{rlrl}
\Delta w_{s 1}(0) & =0 & & \lambda_{11}\left(t_{f}\right)=0 \\
\Delta w_{\text {bus }}(0) & =0 & \lambda_{2}\left(t_{f}\right)=0 \\
\Delta w_{1}(0)=0 & \lambda_{31}\left(t_{f}\right)=0 \\
\Delta w_{2}(0)=0 & \lambda_{32}\left(t_{f}\right)=0 \\
p_{\text {is } 1}(0)=p_{\text {is } 1 o} & \lambda_{41}\left(t_{f}\right)=0 \\
p_{\text {os } 1}(0)=p_{\text {os } 1 o} & \lambda_{51}\left(t_{f}\right)=0 \\
p_{\text {in } 1}(0)=p_{\text {in } 1 o} & \lambda_{61}\left(t_{f}\right)=0 \\
p_{\text {in } 2}(0)=p_{\text {in } 2 o} & \lambda_{62}\left(t_{f}\right)=0 .
\end{array}
$$

The solution of (2.21) and (2.22) yields the game-theoretic control trajectories for the stored 
energies $w_{s}, w_{b u s}, w_{1}$ and $w_{2}$, the power flows $p_{i s 1}, p_{o s}, p_{i n 1}$ and $p_{i n 2}$ under a transient disturbance, such as a step change in the load, $R_{1}, R_{2}$ or both. This is a generalized solution in the sense that it solves for power flows and energy stored in the microgrid. It is thus possible to formulate a problem for any transient disturbance in the system and use these trajectories in different situations.

\subsubsection{Construction of the game-theoretic manifold in the energy-power domain}

To construct a geometric surface, the example system of Fig. 2.2 is subjected to a series step changes in load. First, $R_{1}$ is given a step change from its initial value of $50 \Omega$ to $49 \Omega$

and $R_{2}$ is kept at its initial value of $25 \Omega$. For this case, a set of trajectories for each of the source converter and the POLCs are obtained. Next, a step from $50 \Omega$ to $48 \Omega$ is given to $R_{1}$ while $R_{2}$ is kept constant. Again, a new set of trajectories are obtained. In a similar manner, keeping $R_{2}$ constant, a series of trajectories are obtained by stepping $R_{1}$ at increments of $1 \Omega$ from $15-100 \Omega$. Similarly, set of trajectories are obtained when $R_{1}$ is kept constant and steps at increments of $1 \Omega$ from $12-100 \Omega$ are applied to $R_{2}$. Next, steps are applied to both $R_{1}$ and $R_{2}$ simultaneously to generate another set of trajectories. The range of the step changes and the sample system parameters on which this derivation is done are shown in Table 2.1. 
Table 2.1

System parameters for the solution of the game-theoretic control problem in the example dc microgrid

\begin{tabular}{|c|c|c|c|c|c|c|c|c|}
\hline$V_{d c 1}$ & $\mathrm{~L}$ & $\mathrm{C}$ & $R_{1}$ & $R_{2}$ & $w_{\text {s1nom }}$ & $w_{\text {busnom }}$ & $w_{1 \text { nom }}$ & $w_{2 \text { nom }}$ \\
\hline $1-11 \mathrm{~V}$ & $1 \mathrm{mH}$ & $100 \mathrm{mF}$ & $15-100 \Omega$ & $12-100 \Omega$ & $7.2 \mathrm{~J}$ & $7.2 \mathrm{~J}$ & $28.8 \mathrm{~J}$ & $16.2 \mathrm{~J}$ \\
\hline
\end{tabular}

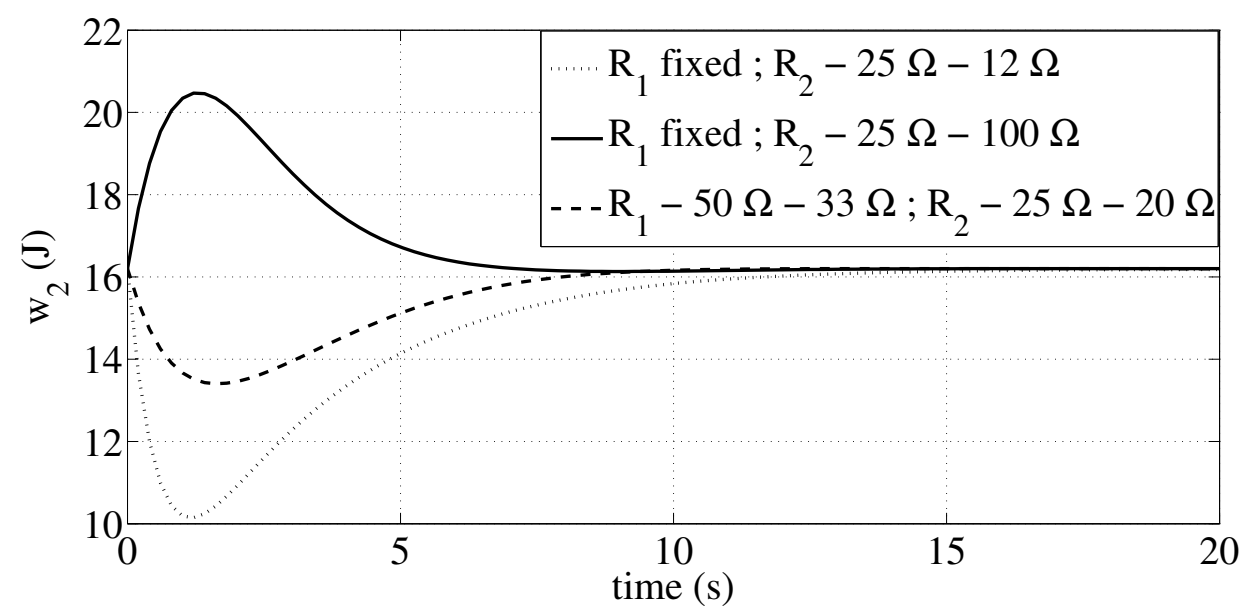

Figure 2.3: POLC 2 stored energy time-domain simulations for three different cases illustrating the formation of the geometric manifolds in the dc microgrid.

The trajectories for power and energy of the POLC 2 subject to three different load step cases are shown in Fig. 2.3 and 2.4, respectively to show how individual trajectories are derived. The load $R_{1}$ is kept constant at $50 \Omega$ and $R_{2}$ is varied from $25 \Omega$ to $12 \Omega$ and $25 \Omega$ to $100 \Omega$ for the first two cases. For the third case load $R_{1}$ is varied from $50 \Omega$ to $33 \Omega$ and $R_{2}$ is varied from $25 \Omega$ to $20 \Omega$.

For a larger set of cases the trajectories can be combined together to generate the complete manifold. These complete manifolds for the system in Fig. 2.2 are shown in Fig. 2.5, 2.6 and 2.7. Fig. 2.5 shows the two-dimensional surface comprising of the energy and power states of the source converter 1 with parameters from Table 2.1. This surface consists of 


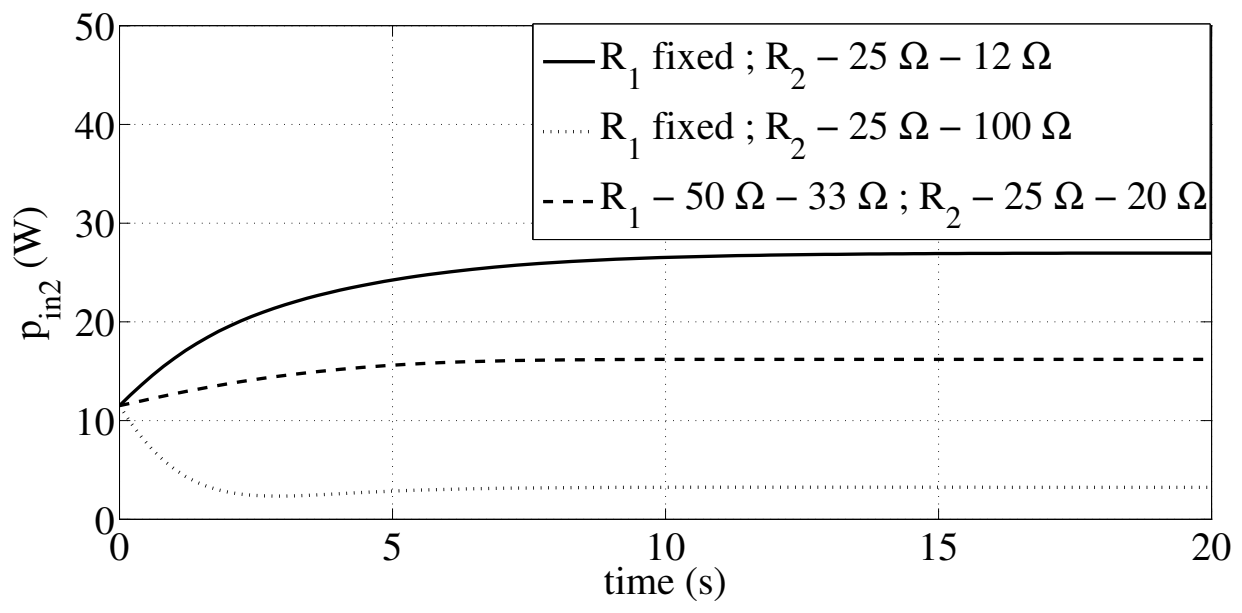

Figure 2.4: POLC 2 input power time-domain simulations for three different cases illustrating the formation of the geometric manifolds in the de microgrid.

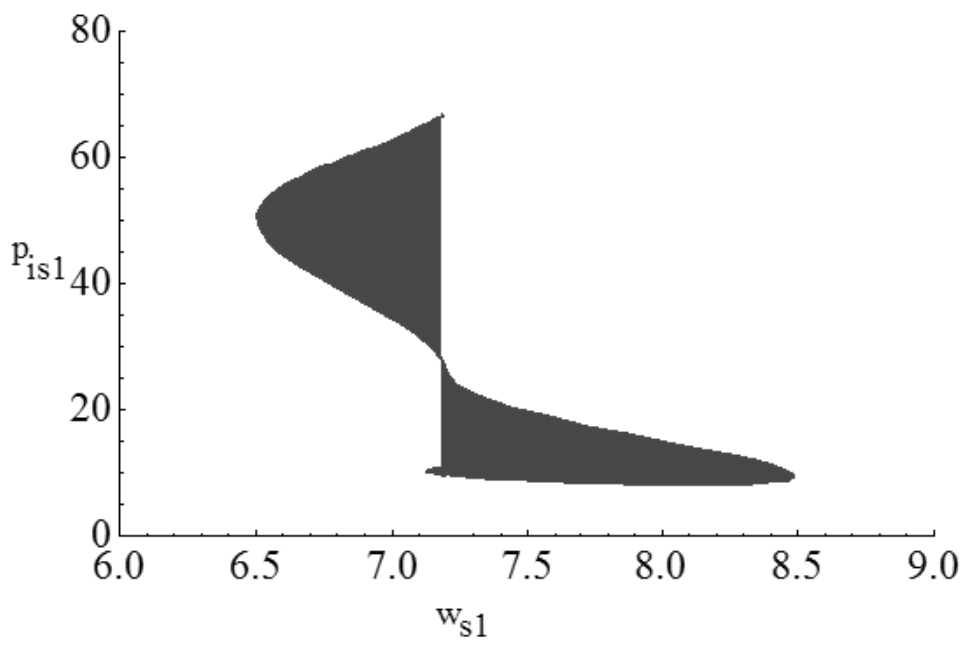

Figure 2.5: Source converter geometric manifold in the energy-power domain for the example dc microgrid.

the individual state trajectories of the source converter 1 for the ranges of step changes in load shown in Table 2.1. Figs. 2.6 and 2.7 show the resulting surfaces for the two POLCs. The POLC surfaces are three-dimensional with the energy stored in the bus capacitor as 


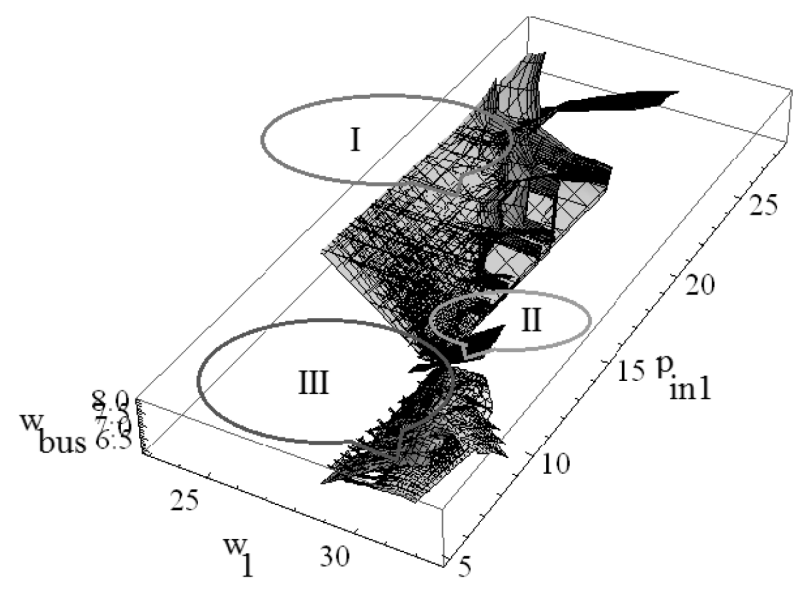

Figure 2.6: POLC 1 geometric manifold in the energy-power domain for the example dc microgrid.

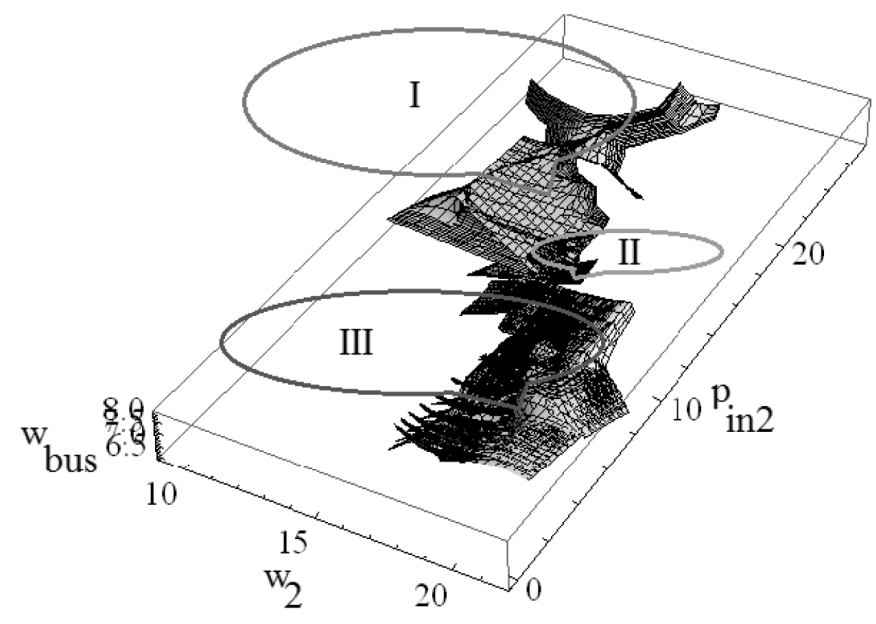

Figure 2.7: POLC 2 geometric manifold in the energy-power domain for the example dc microgrid.

the third dimension, whereas the source converter surface is only two-dimensional as its objective is to only regulate internal energy, $w_{s 1}$, and this is same as the stored energy in the bus capacitor. Fig. 2.6 can be divided into three different regions based on the load 
condition. In Fig. 2.6, region I shows the manifold comprising of the state trajectories when there are step changes in one or both the loads and the load power demand on POLC 1 increases. Region II shows the state trajectories of POLC 1 when there is a step change in $R_{2}$ only, thus the load power demand on POLC 1 stays the same. Region III shows the collection of the state trajectories of POLC 1 when there are step changes in one or both the loads and the load power demand on POLC 1 decreases. In a similar manner, Fig. 2.7 can be divided into three different regions based on the load condition. Region I shows the region of the manifold comprising of the state trajectories when there are step changes in one or both the loads and the load power demand on POLC 2 increases. Region II shows the state trajectories of POLC 2 when there is a step change in $R_{1}$ only, thus the load power demand on POLC 2 stays the same. Region III shows the collection of the state trajectories of POLC 2 when there are step changes in one or both the loads and the load power demand on POLC 2 decreases.

These surfaces will act as the basis for the controller as the reference surface and only requires local sensor information, thus eliminating the need for a communications structure. These surfaces are derived based on a finite set of load step scenarios. It is anticipated that surfaces derived from small sets of cases can span the space for larger sets. Sections 2.3 and 2.4 address the use of the derived surfaces in unknown situations and in larger event sets. Note that this example only shows the trajectories for one source converter and two POLCs, but the approach is valid for any generic system with $n$ source converters and $m$ POLCs. The step change in the source will have no effect on the trajectories in the energy-power 
domain, since the concern here is to solve the power in-flows and energies stored only. Once the input power and energy stored states are solved, then they will be converted to input current and terminal voltage states, where the effect of the step change in the source will become visible.

\subsubsection{Conversion of the game-theoretic manifolds to the voltage-current domain}

The manifolds derived in the previous section is based on the energy stored in the power converters and the power flows in the de microgrid as shown in Fig. 2.1 and 2.2. The energy-power domain is for derivation purposes only. To be implemented in hardware, the manifolds in the energy and power flow states are mapped to current and voltage states. The system in Fig. 2.1 is modified to show the system in terms of voltage and current states and is shown in Fig. 2.8

In Fig. 2.8, $V_{d c i}, i \in \mathscr{N}$, are the input voltages to the system. The solutions to the game-theoretic control problem is obtained from (2.19) and (2.20) and the stored energies $w_{s i}, w_{b u s}$ and $w_{j}$ and the power flows $p_{i s i}, p_{o s i}$ and $p_{i n j}$ are converted into voltage and current states as shown in 2.23. Here, the effects of step changes in the source will be 


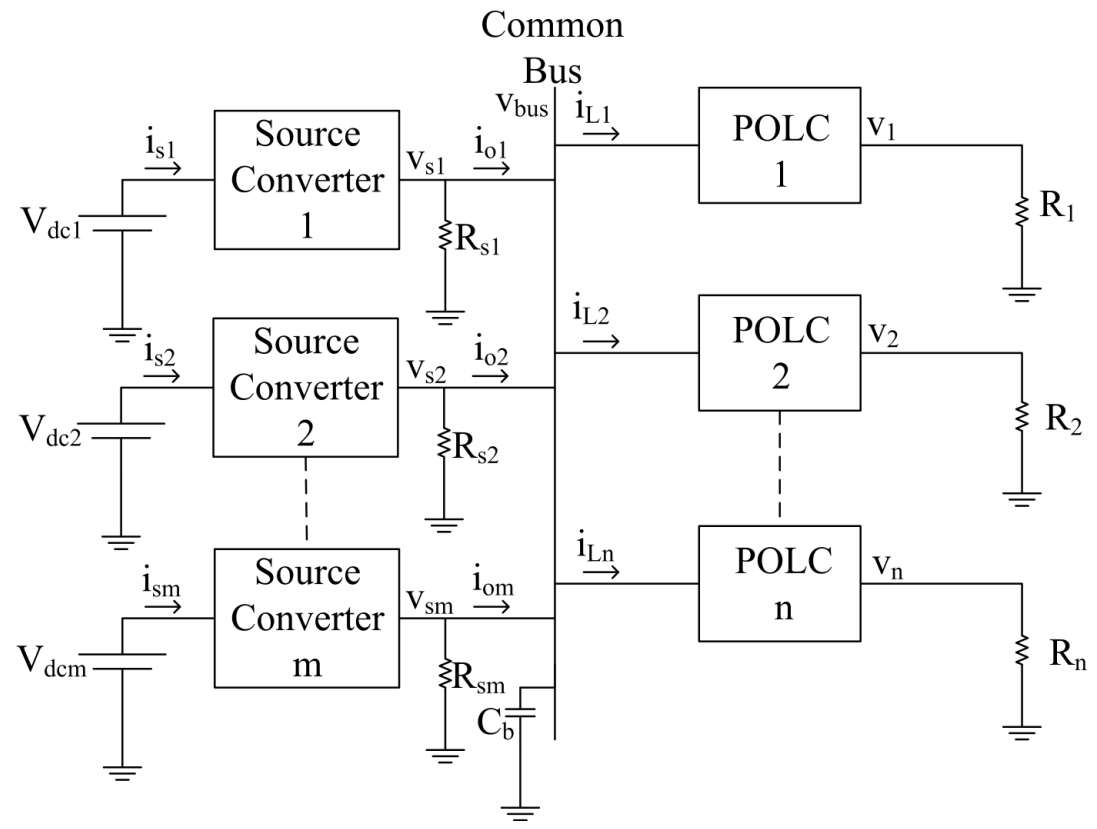

Figure 2.8: General dc microgrid with $n$ source converters and $m$ POLCs in the voltage-current domain. Adapted from Fig. 8 of [1].

reflected in the input current states of the source converters.

$$
\begin{aligned}
& v_{s i}=\sqrt{\frac{2 w_{s i}}{C_{s i}}} \\
& v_{\text {bus }}=\sqrt{\frac{2 w_{\text {bus }}}{C_{b}}} \\
& v_{j}=\sqrt{\frac{2 w_{j}}{C_{j}}} \\
& i_{s i}=\frac{p_{\text {isi }}}{V_{d c i}} \\
& i_{\text {osi }}=\frac{p_{\text {osi }}}{v_{\text {bus }}} \\
& i_{L j}=\frac{p_{\text {inj }}}{v_{\text {bus }}}
\end{aligned}
$$

For the example system considered in the previous section, Fig. 2.8 can be redrawn for $n=1$ and $m=2$ as shown in Fig. 2.9. From Fig. 2.9, the conversions from (2.23) are 


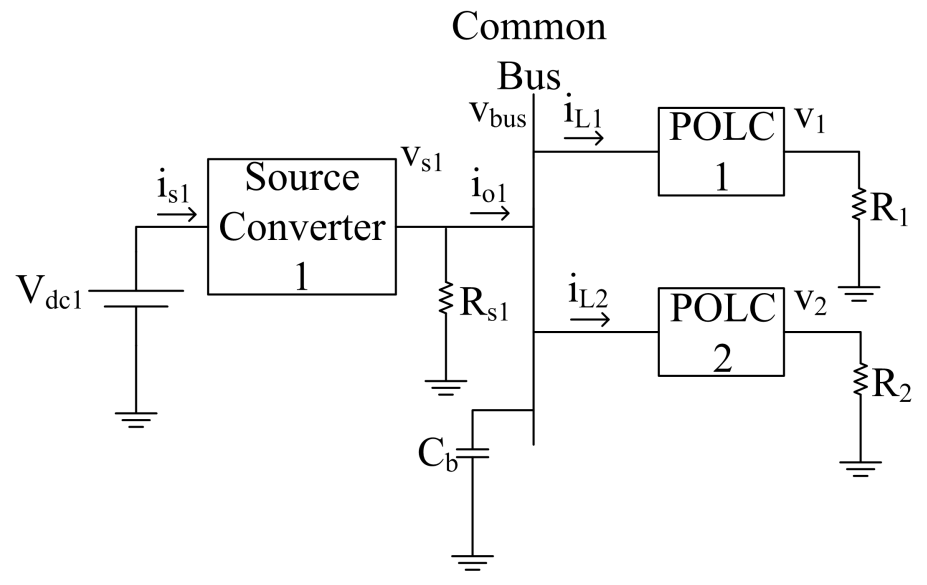

Figure 2.9: Example dc microgrid with '1' source converter and '2' POLCs in the voltage-current domain. Adapted from Fig. 9 of [1].

written as

$$
\begin{aligned}
& v_{s 1}=\sqrt{\frac{2 w_{s 1}}{C_{s 1}}} \\
& v_{\text {bus }}=\sqrt{\frac{2 w_{\text {bus }}}{C_{b}}} \\
& v_{1}=\sqrt{\frac{2 w_{1}}{C_{1}}} \\
& v_{2}=\sqrt{\frac{2 w_{2}}{C_{2}}} \\
& i_{s 1}=\frac{p_{\text {is } 1}}{V_{\text {dc } 1}} \\
& i_{o s 1}=\frac{p_{\text {os } 1}}{v_{\text {bus }}} \\
& i_{L 1}=\frac{p_{\text {in } 1}}{v_{\text {bus }}} \\
& i_{L 2}=\frac{p_{\text {in } 2}}{v_{\text {bus }}} .
\end{aligned}
$$

The geometric manifolds shown in Fig. 2.5 through 2.7 are converted to the voltage-current domain for the same example system and are shown in Fig. 2.10 through 2.12

Fig. 2.10 is now a three dimensional surface derived from Fig. 2.5, the source voltage 


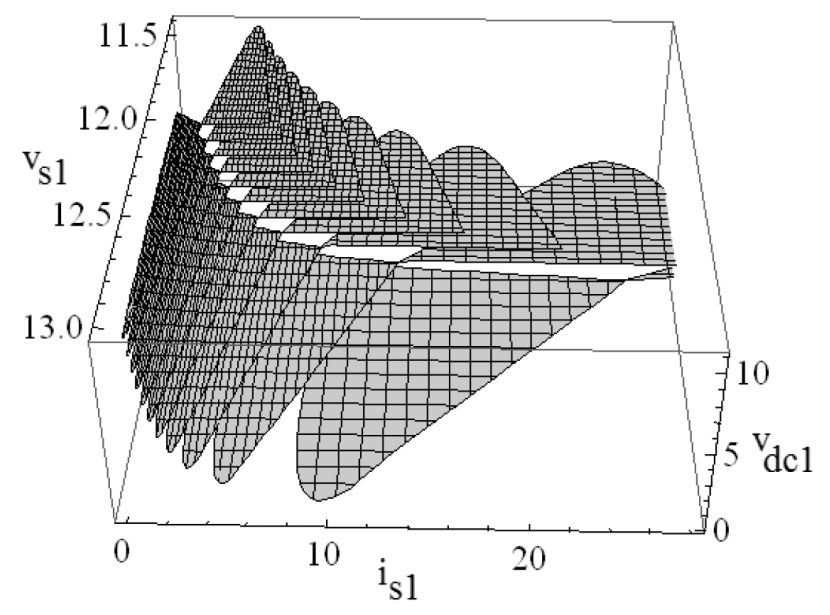

Figure 2.10: Source converter surface in the voltage-current domain for the example dc microgrid.

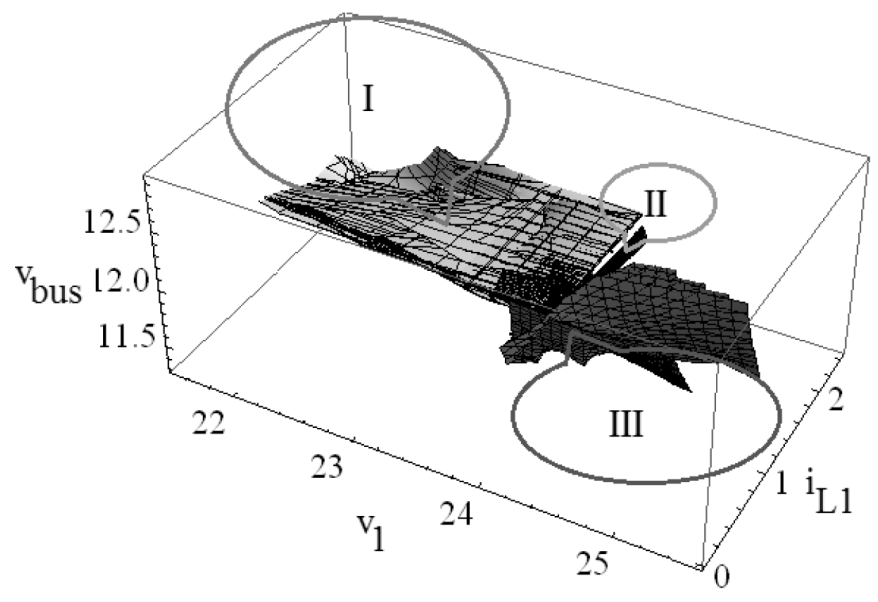

Figure 2.11: POLC 1 surface in the voltage-current domain for the example dc microgrid.

being the third dimension. This is a modification over the surface for the source converter in the current-voltage domain as shown in [1]. The source voltage is stepped at increments 


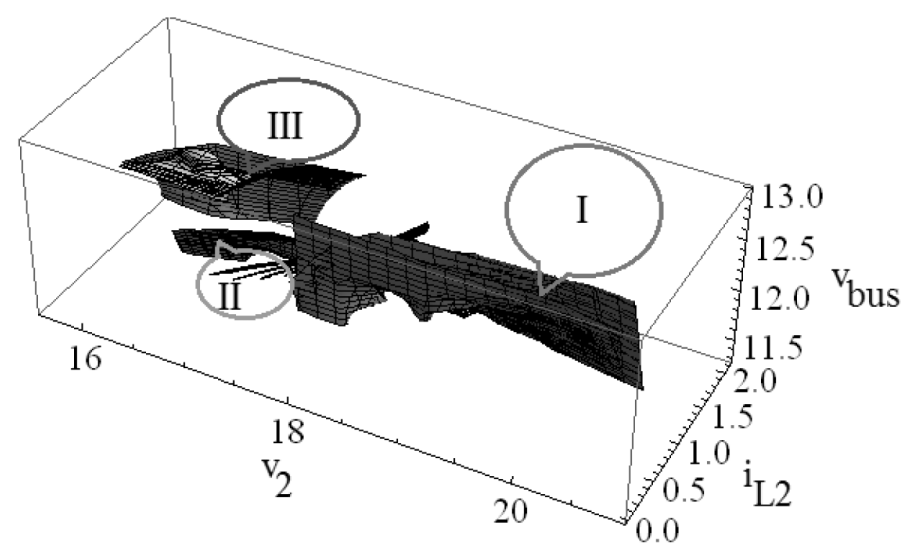

Figure 2.12: POLC 2 surface in the voltage-current domain for the example dc microgrid.

of $1 \mathrm{~V}$ for a range of $1-11 \mathrm{~V}$. The different layers in Fig. 2.10 shows the surfaces for different source voltages. Fig. 2.11 and 2.12 shows the three dimensional surfaces for the POLCs in the voltage-current domain. Fig. 2.11 can be divided into three different regions based on the load condition. Region I shows the part of the manifold comprising of the state trajectories when there are step changes in one or both the loads and the load power demand on POLC 1 increases. Region II shows the state trajectories of POLC 1 when there is a step change in $R_{2}$ only, thus the load power demand on POLC 1 stays the same. Region III shows the collection of the state trajectories of POLC 1 when there are step changes in one or both the loads and the load power demand on POLC 1 decreases. Similarly, Fig. 2.12 can be divided into three different regions based on the load condition. Region I shows the part of the manifold comprising of the state trajectories when there are step changes in one or both the loads and the load power demand on POLC 2 increases. Region II shows 
the state trajectories of POLC 2 when there is a step change in $R_{1}$ only, thus the load power demand on POLC 2 stays the same. Region III shows the collection of the state trajectories of POLC 2 when there are step changes in one or both the loads and the load power demand on POLC 2 decreases.

The POLCs respond to the changes in the system and get the local information from the loads. The step changes in the load compels the POLCs to make necessary changes according to the system control objectives. The step changes in the load changes the output impedance of the source converter. Based on the output impedance as seen by the source converter, it makes necessary changes according to its control objective. Thus, the manifolds in Fig. 2.10 through 2.12 are the practical geometric manifolds for a range of step changes in the loads and sources specified in Table 2.1 .

\subsection{Simulation results}

To test the strategy for the example three converter system in Fig. 2.9, a simulation is done in MATLAB/Simulink. The reference surfaces derived in Section2.1.3 and used as a-priori calculated surfaces and fed into a memory lookup table. This memory table contains the surfaces and the switching law. The switch states for the converters are generated based on the sliding mode hysteretic control technique [29, 32, 87, 88]. Therefore, the reference surfaces pertaining to different transient scenarios, that form the complete manifold, are 
the sliding surfaces in the controller. The system sensor state feedback is fed into the memory table. Based on these system state trajectories, the memory lookup table control law determines the switch states for each power converter. The switch states of each power converter are the outputs of the memory lookup table. The purpose of the controller is to drive the system states to the surfaces stored in the memory location.

The load $R_{1}$ is first given a step from $50 \Omega$ to $33 \Omega$ and the load $R_{2}$ is given a step from $25 \Omega$ to $20 \Omega$. With reference to Fig. 2.9, the initial and final system parameters selected are shown in Table 2.2 and Table 2.3 respectively. The converters considered for the purpose are all dc-dc boost converters. Next a step change in the source voltage is given from $9 \mathrm{~V}$ to $6 \mathrm{~V}$. The final system parameters for this transient condition is shown in Table 2.4 .

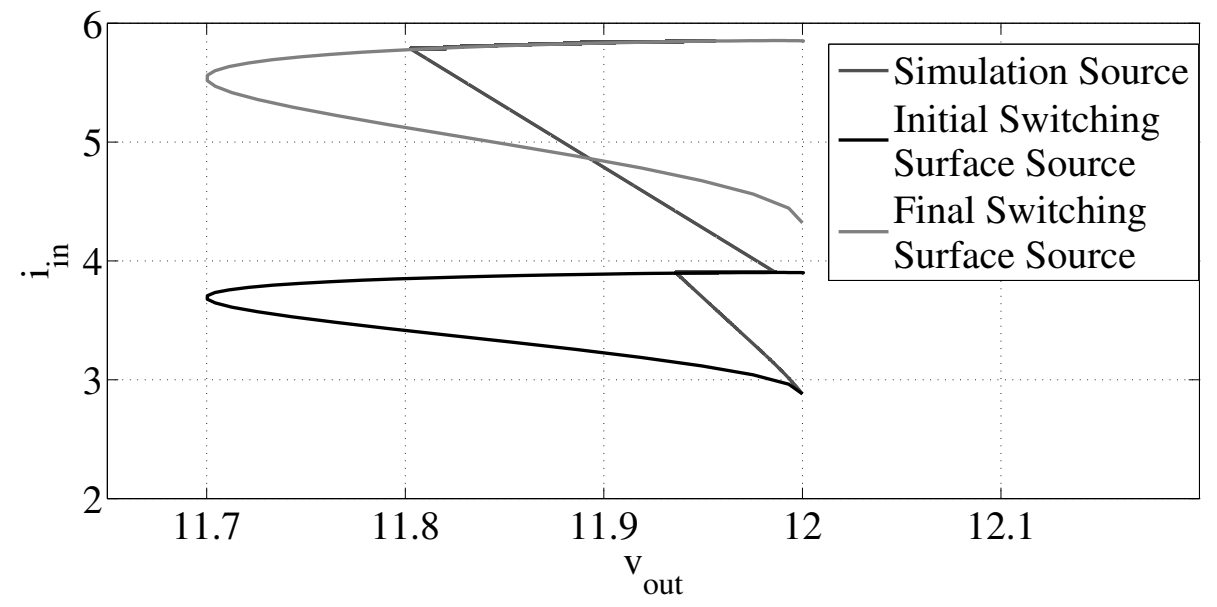

Figure 2.13: State Trajectories for the source converter for the example dc microgrid used in the simulation. 
Table 2.2

Example dc microgrid initial parameters used in the simulation.

\begin{tabular}{|c|c|c|c|c|c|c|c|c|c|c|c|}
\hline$V_{d c 1}$ & $\mathrm{~L}$ & $\mathrm{C}$ & $V_{s 1}$ & $V_{b}$ & $V_{1}$ & $V_{2}$ & $i_{s 1}$ & $i_{L 1}$ & $i_{L 2}$ & $R_{1}$ & $R_{2}$ \\
\hline $9 \mathrm{~V}$ & $20 \mathrm{mH}$ & $100 \mathrm{mF}$ & $12 \mathrm{~V}$ & $12 \mathrm{~V}$ & $24 \mathrm{~V}$ & $18 \mathrm{~V}$ & $2.88 \mathrm{~A}$ & $0.96 \mathrm{~A}$ & $1.08 \mathrm{~A}$ & $50 \Omega$ & $25 \Omega$ \\
\hline
\end{tabular}

Table 2.3

Example dc microgrid final parameters for a step change in load used in the simulation.

\begin{tabular}{|c|c|c|c|c|c|c|c|c|c|c|c|}
\hline$V_{d c 1}$ & $\mathrm{~L}$ & $\mathrm{C}$ & $V_{s 1}$ & $V_{b}$ & $V_{1}$ & $V_{2}$ & $i_{s 1}$ & $i_{L 1}$ & $i_{L 2}$ & $R_{1}$ & $R_{2}$ \\
\hline $9 \mathrm{~V}$ & $20 \mathrm{mH}$ & $100 \mathrm{mF}$ & $12 \mathrm{~V}$ & $12 \mathrm{~V}$ & $24 \mathrm{~V}$ & $18 \mathrm{~V}$ & $3.90 \mathrm{~A}$ & $1.45 \mathrm{~A}$ & $1.35 \mathrm{~A}$ & $33 \Omega$ & $20 \Omega$ \\
\hline
\end{tabular}

Table 2.4

Example dc microgrid final parameters for a step change in source used in the simulation.

\begin{tabular}{|c|c|c|c|c|c|c|c|c|c|c|c|}
\hline$V_{d c 1}$ & $\mathrm{~L}$ & $\mathrm{C}$ & $V_{s}$ & $V_{b}$ & $V_{1}$ & $V_{2}$ & $i_{s} 1$ & $i_{L 1}$ & $i_{L 2}$ & $R_{1}$ & $R_{2}$ \\
\hline $6 \mathrm{~V}$ & $20 \mathrm{mH}$ & $100 \mathrm{mF}$ & $12 \mathrm{~V}$ & $12 \mathrm{~V}$ & $24 \mathrm{~V}$ & $18 \mathrm{~V}$ & $5.85 \mathrm{~A}$ & $1.45 \mathrm{~A}$ & $1.35 \mathrm{~A}$ & $33 \Omega$ & $20 \Omega$ \\
\hline
\end{tabular}

The system undergoes an initial transient in the form of a step change of both the loads $R_{1}$ and $R_{2}$. Then it undergoes another transient situation in the form of step change in the source voltage. The complete geometric surfaces for these scenarios are pre-calculated and stored in the memory tables along with the respective switch states prior to the system model simulation. The system trajectories follow the sliding surfaces when the transient conditions are simulated. The state trajectories reach the new equilibrium points based on the predetermined sliding manifold as fed into the memory table. Fig. 2.13 shows the geometric surfaces based on the a-priori calculations for the source converter for this example case, along with the state trajectories generated in the simulation. The surfaces are slices of the complete manifold pertaining to the specific transient cases considered in this example. The simulation trajectory reaches these particular slices and hence it stays on the complete manifold. Therefore, it may be concluded that the system states can be driven 
to the required sliding surface based on the a-priori geometric surface calculations. Hence, the complete geometric manifolds or parts of them can be effectively used as reference surfaces for a controller.

\subsection{Limits of applicability of the method and response in unknown situations}

The method described in Section 2.1.1 is a generalized method for $n$ source converters and $m$ POLCs. The derivation considered only power flows and energy stored as state variables. The problem formulation using this kind of an energy-balance approach makes this a generic method since the choice of the power converter topology becomes immaterial. The example system used for the derivation of the manifolds uses dc-dc boost converters. However, in a general sense, any type of converter topology may be used.

Any real-time system has a specific design criteria. This would include the maximum current and voltage ratings, load conditions, etc. The solution for the game-theoretic control problem used for the example case in this work is based on the system parameters specified in the Tables 2.1 to 2.4 . The surfaces are derived based on these parameters. However, if the system specifications change, then the parameters will change, but the method of derivation of the surfaces will remain the same. 
The derivation and simulation in Section 2.2 assumes initial state equilibrium points for all the converters in the system. However, these reference surfaces can be used, without any modification, for situations where the initial state equilibrium points are different. As an illustration, a simulation is done with the same system parameters and same control method as described in Section 2.2. However, the initial equilibrium point for the source converter is different from that used for the derivation. The source converter has an initial equilibrium point of $(15 \mathrm{~V}, 2.97 \mathrm{~A})$ instead of $(12 \mathrm{~V}, 2.88 \mathrm{~A})$. Fig. 2.14 shows that state trajectory for the source converter slides along the reference surface and reaches the final equilibrium point. This illustrates the applicability of the reference surface in controllers for a situation not explicitly used to derive the surfaces.

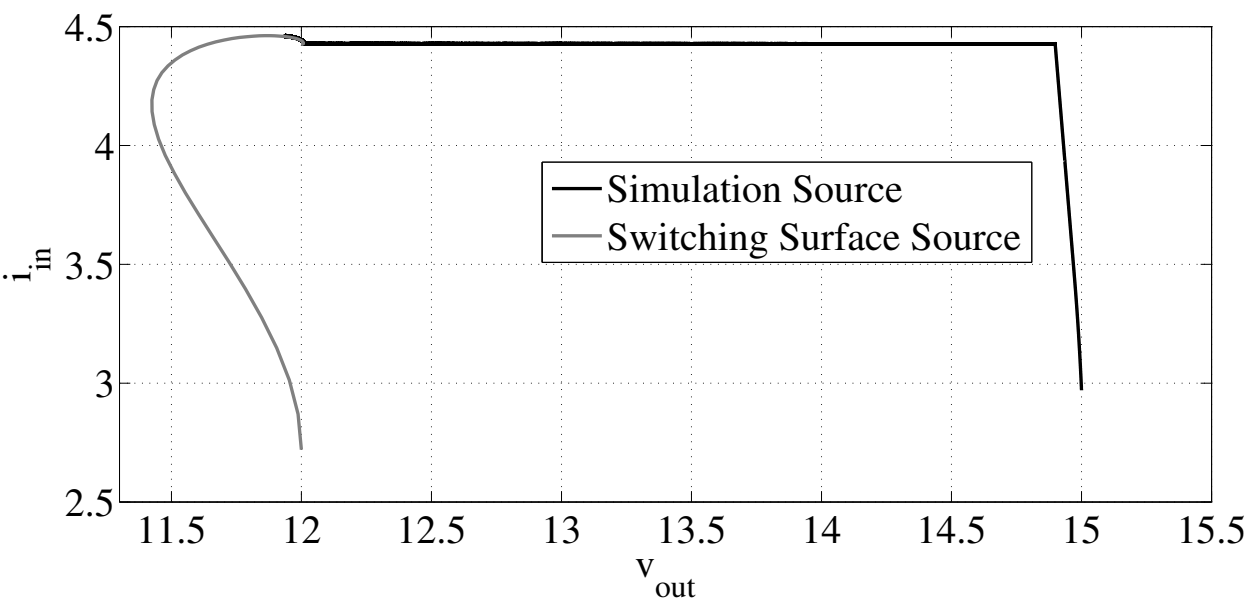

Figure 2.14: State Trajectories for the source converter for example dc microgrid used in an unknown situation. 


\subsection{Discussions}

Equation (2.10) shows the objective function used for the derivation of the manifolds. If a different objective is chosen, then (2.10) needs to be modified and the same method of derivation can be used to generate the manifolds for that system. For the example system, a granularity of 1 unit in the step changes in source and load are considered. If necessary, this granularity can be decreased to incorporate more precise events. But within the specified ranges, the surfaces for any load or source condition, not used for the derivation, can be generated and used as reference surfaces for the controller. This is because they already lie on the generated manifolds.

For the example system, the lower limit for the resistances are chosen in such a manner that it does not demand more power from the system than it can deliver. This would result in a power mismatch in the system. Similarly, the lower limit of the source voltage needs to be chosen such that it is capable of transfering enough energy to the loads. The upper limits for the load can be anything as long as some current flows through them. The upper limit for the source voltage depends on the converter topology used and the terminal voltage required at the source converter end. The derivation has been done for two transient conditions - step changes in the loads and source. Other transient conditions, such as a startup may also be included in the derivation. The simulation done in Section 2.3 shows an example where the reference surface can be used in a situation not explicitly used to derive the surfaces. 


\subsection{Conclusion}

The game-theoretic trajectories for the power converters in a microgrid have been derived for various events, such as step changes in the load and source. The importance of these trajectories and the complete geometric manifold they form lie in the fact that they can be used as reference surfaces for any controller. The switch states of the power converters need to be controlled by a controller and these surfaces will act as a basis of the control. The manifolds are still limited to the specific ranges of load and source steps considered. However, this method may be extended to any range of loads and is applicable for any number of energy storage devices in a microgrid. 


\section{Chapter 3}

\section{Digital sliding mode hysteretic control in}

\section{a dc-dc boost converter}

In [34], digital implementation of a hysteretic sliding mode control strategy is shown by implementing a predetermined surface inside a DSP. The DSP mentioned in this work is a Texas Instruments TMS320F2812 DSP where the controller was programmed in Simulink. This chapter uses the work in [34] as the starting point and designs and implements a digital sliding mode hysteretic control method of switching converters in a dc-dc boost converter, where the sliding surfaces are predetermined and stored in a low-cost memory circuit instead of using any form of software component in the design. It can also be shown that by using lower cost and slower analog-to-digital conversion (ADC) in the process, the control can be improved by eliminating the need to derive and implement a hysteresis band 
which limits the effective switching frequency [84]. A stability analysis for the surfaces chosen for the example system is also provided.

\subsection{Implementation of the digital hysteretic sliding mode controller in real time}

In microgrids, the use of digital control in switching power converters has increased due to the decreased cost of digital controllers, such as microcontrollers, DSP and EEPROM [33]. A predetermined calculation of the reference surface can be used in conjunction with a digital controller in the controller design. This is beneficial in the sense that on-site calculations are not necessary thus making the controller less complicated and much faster. Different choices of reference surfaces can be calculated a-priori and fed into a memory lookup table. This memory table contains the complete surface and the switching law. The memory table thus constitutes the digital part of the control system. The system sensor state feedback is fed into the memory table. The surfaces which are stored in the memory table are converted into quantized surfaces. Based on these quantized system state trajectories, the memory table control law determines the switch states of each power converter. The switch states of each power converter are the outputs. The purpose of the controller is to drive the system states to the quantized surfaces stored in the memory location. This memory location thus forms the control center for the overall controller. The block diagram 
of a simple microgrid with a source, a load and a POLC, along with the memory lookup table/EEPROM is shown in Fig. 3.1. Here the inputs to the converter, which is a dc-dc boost converter, are the input voltage and the output current. The output voltage and the input current are fed into the memory table via analog to digital converters. The memory table which has the switching law incorporated in it generates the switch state which is fed into the converter thereby controlling the system states.

A local information is also fed into the memory table. This information contains the condition of the load for the POLC and the memory table associated to the POLC is programmed to respond to a change in the system, such as a step change in the load. Thus, this kind of a controller is capable of generating a correct switch state based on the system conditions. The switching for the converters are controlled by the hysteretic sliding mode control technique [89]. Thus, if a predetermined sliding surface, along with the hysteretic sliding mode controller is fed into a memory location prior to a system operation, this kind of a digital hysteretic sliding mode control strategy is capable to drive the system states to the desired equilibrium points based on the control. 


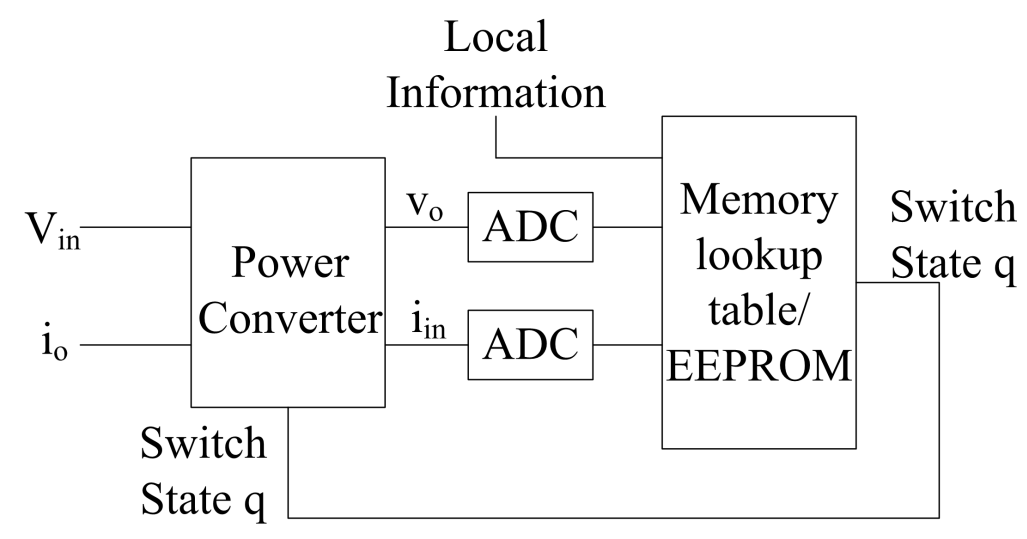

Figure 3.1: Digital memory lookup based controller block diagram for the dc-dc boost converter. Adapted from Fig. 13 of [1].

\subsection{Experimental apparatus}

Analog signals from the power stage of the converter are converted into digital signals of $m$ bits using ADCs. The ADCs have a parallel interface. The digital output of the ADCs is interfaced with a parallel EEPROM chip inside which, the switching surfaces are stored based on the open-loop solutions for different scenarios. Between two parallel digital devices, an entire byte of data is transmitted at the same time. Therefore, when information from the analog signal of the power stage is converted to m-bit digital signal at the output of the ADC, the entire m-bit of data is transmitted to the parallel EEPROM at the same time. The physical address space is used as n-dimensional (where $n$ is the number of analog signals being fed into ADC converters) look-up table and the data outputs from the memory chip are used as switch states for the control of switching power converters for different transient events. 
This control scheme will have a very fast response time if ADC converters with appropriate resolution and fast sampling rate EEPROMs are used. The performance of the ADC conversion process can be judged based on its resolution in bits per sample, signal-to-noise ratio (SNR), spurious-free dynamic range (SFDR), power dissipation, and quantization error [90, 91]. For implementing the hysteretic sliding-mode control of the power converters, an 8 bit ADC converter is used for each analog signal. The effects of SNR, SFDR, and power dissipation of ADC converters have been ignored in this work. The block diagram of this set-up is shown in Fig. 3.1. This is a generic hardware set-up that can be used to control a switching power converter with any number of state-analog signals.

\subsection{Memory resolution analysis}

Fig. 3.2 shows the circuit of the system used for the memory resolution analysis. This is a dc-dc boost converter circuit with a digital controller. The dynamic equations for the system is

$$
\begin{aligned}
& L \dot{i}_{\text {in }}=V_{\text {in }}-(1-q) v_{\text {out }} \\
& C \dot{v}_{\text {out }}=(1-q) i_{\text {in }}-\frac{v_{\text {out }}}{R}
\end{aligned}
$$

where $i_{\text {in }}$ is the input current, $v_{\text {out }}$ is the output voltage, $V_{\text {in }}$ in the input voltage, $q$ is the switch state, $\mathrm{L}$ and $\mathrm{C}$ are energy storage elements and $\mathrm{R}$ is a constant resistive load. 


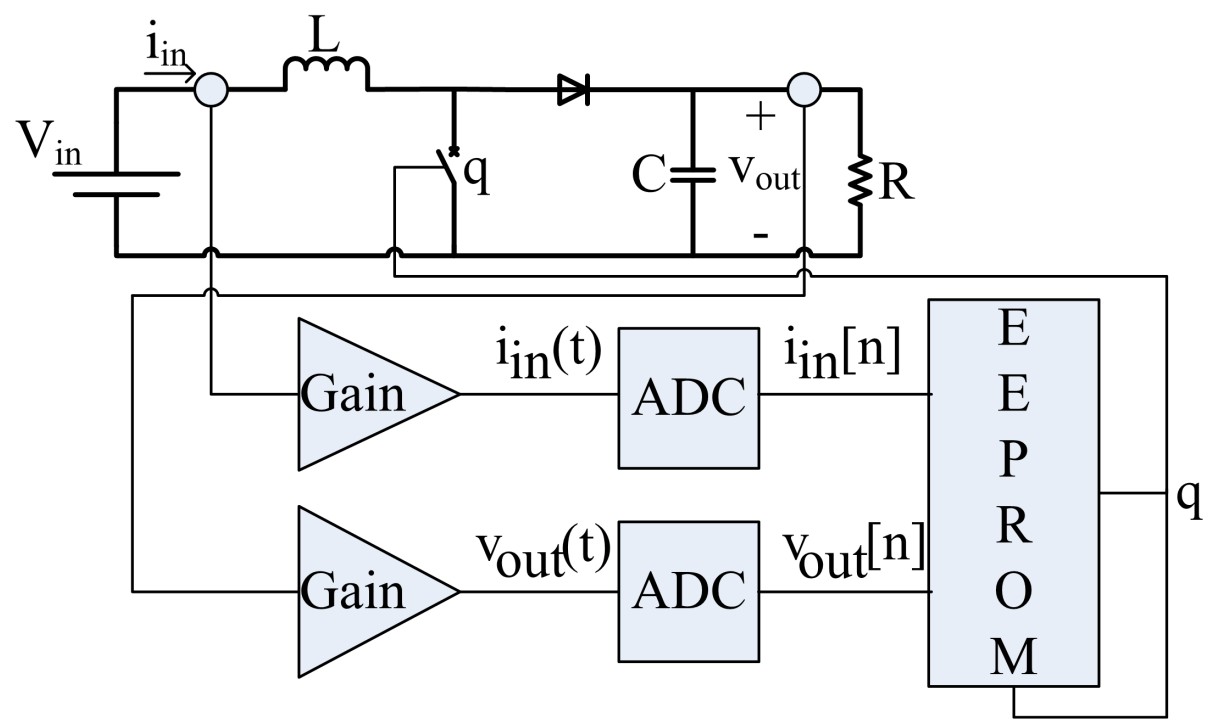

Figure 3.2: Circuit for the implementation of the digital sliding mode control strategy with two ADCs and a single memory lookup table in a dc-dc boost converter for resolution analysis.

The state space representation of (3.1) gives

$$
\dot{x}(t)=\left(\begin{array}{cc}
0 & -\frac{1-q}{L} \\
\frac{1-q}{C} & -\frac{1}{C R}
\end{array}\right) x(t)+\left(\begin{array}{c}
\frac{V_{i n}}{L} \\
0
\end{array}\right)
$$

where

$$
x(t)=\left(\begin{array}{c}
i_{\text {in }}(t) \\
v_{\text {out }}(t)
\end{array}\right)
$$

The system model depends on the system parameters $\lambda\left(\mathrm{R}, \mathrm{L}, \mathrm{C}\right.$ and $\left.V_{\text {in }}\right)$, the states $x(t)$ and the switch state $q$. The switch state is generated from a digital hysteretic sliding 
mode controller. The system state trajectories are first attracted towards a reference sliding surface $s(x)$, based on the switching control law defined in (1.2). Then they travel along $s(x)$ and finally limit cycles about the intersection of $s(x)=0$ and the desired steady state operating point $x_{e}\left(v_{o h}, i_{L o h}\right)$, within a band $h$, known as the hysteresis band.

A dc-dc boost converter has a range of operating points depending on $\lambda$ and $x(t)$. The load line is given by

$$
i_{\text {in }}=\frac{v_{\text {out }}^{2}}{R V_{\text {in }}} .
$$

The sliding surface chosen for this analysis is a sloped linear surface in the state-plane. The reason for the choice of this surface is discussed in Section 3.5.2. The equation for the surface is

$$
s=i_{\text {in }}-m-b v_{\text {out }}
$$

where $\mathrm{m}$ is the $i_{i n}$-axis intercept and $\mathrm{b}$ is the slope of the line. The slope of the line is determined by

$$
b=\frac{M}{v_{o h}}-\frac{v_{o h}}{R V_{\text {in }}}
$$

where $v_{o h}$ is the desired voltage operating point. The intersection of $s(x)$ and the load line gives the steady state operating point around which the states limit cycles. The digital 
controller consists of ADCs and a memory lookup table.

The surface shown in (3.5) is a function of time and the states, assuming the $i_{\text {in }}$ intercept constant. The surface is also a function of system parameters $\lambda$, since the slope $b$ is dependent on $R$ and $v_{o h}$. The surface can be constructed a-priori in a memory lookup table with open-form scaled state values in the form of a table called raster surface. In the system shown in Fig. 3.2, the ADCs convert the state signals $x(t)$ into sampled signals $x[n]$. The sampling resolution steps depend on the no. of bits of the ADC. If the number of ADC bits are $M$, the number of resolution steps is $2^{M}$. The number of resolution steps give the number of memory addresses needed for the surface generation. Therefore, in the surface generation process, scaling of the analog signals to sampled signals is required. These sampled signals $x[n]$ form the pixels of the surface. The complete surface is generated by many such pixels taken together. This surface can further be divided into two separate regions based on the switch state. The pixels are compared to (3.5) and depending on whether they are greater than or less than zero, forms the $s(x)>0(O N)$ and $s(x)<0(O F F)$ regions. The surface generated so far do not include the hysteresis bands in it. To generate the hysteresis bands, the switch state feedback to the memory table, as shown in Fig. 3.2 , is necessary. If the switch state is $1(\mathrm{ON})$, the pixels are compared to $s(x)-\frac{h}{2}$, and if it is 0 (OFF), the pixels are compared to $s(x)+\frac{h}{2}$, where $h$ is the required hysteresis band. All pixels that satisfy $s(x)+\frac{h}{2}<0$ forms the $O N$ region and all pixels that satisfy $s(x)-\frac{h}{2}>0$ forms the $O F F$ region. It may be noted that there is a region of overlap. This region of overlap forms the hysteresis band in the memory. Fig. 3.6 shows the full surface in 
the memory comprising of all the pixels. The light pixels form the $O N$ region, the darker pixels form the $O F F$ region, and the region of overlap, shown by the darkest pixels, is the hysteresis band. These pixels form the addresses of the memory lookup table.

The output of the memory lookup table is the switch state. Corresponding to each address in the memory, a switch state is programmed by an algorithm that compares the pixel for that address to the surface (3.5) and computes $q$ based on (1.2). Therefore, in the memory lookup table, the complete surface is stored as different addresses and each address has a switch state associated to it. Thus, the memory lookup table forms the digital controller that outputs the switch state based on hysteretic sliding mode control technique. A simulation is done in MATLAB/Simulink for the system shown in Fig. 3.2 with system parameters shown in Table 3.1. The system sensor states are sampled in the ADCs. The sampled states from the ADC lookup the switch states from the memory table. This switch state controls the switch of the dc-dc boost converter and drives the system states from a cold start to the desired equilibrium point. Fig. 3.3 to Fig. 3.6 shows simulation results for four different resolution steps. Fig. 3.3 shows the result for a resolution step of 16 per state variable. Here

Table 3.1

Example dc microgrid parameters for a sloped linear reference surface control.

\begin{tabular}{|c|c|c|c|c|c|c|c|}
\hline$V_{\text {in }}$ & $\mathrm{L}$ & $\mathrm{C}$ & $\mathrm{R}$ & Memory resolution step & $\mathrm{m}$ & $\mathrm{b}$ & $v_{o h}$ \\
\hline $6 \mathrm{~V}$ & $1 \mathrm{mH}$ & $100 \mu \mathrm{F}$ & $12.5 \Omega$ & $16 / 32 / 64 / 128$ & 3 & -0.213 & $9 \mathrm{~V}$ \\
\hline
\end{tabular}

only 4 bits of each ADC converter are used. 256 address locations of the memory lookup table are therefore used. A very low resolution step value implies low number of pixels in 
the hysteresis band. The system states have to lookup for the correct switch state from only 256 address locations. This results in improper switch states and the controller is unable to drive the system states to the surface and take them to the desired equilibrium point. In this case, the pixels in the hysteresis band is not a good representation of the actual hysteresis band. Fig. 3.4 shows the result when the resolution step per state variable is 32 . In this case 5 output bits of each ADC converter and 1024 memory locations of the chip are used. Fig. 3.5 shows the result when the resolution step per state variable is 64 . For this case, 6 output bits of each ADC converter and 4096 memory locations of the chip are used. Fig. 3.6 shows the simulation result for a resolution step size 128 per variable. Here, 7 ADC converter output bits are used and 16384 memory locations of the chip are used. It may be noted that as the resolution step per state variable is increased, number of pixels in the hysteresis band increases and they give a better representation of the actual hysteresis band, and in turn better control. Out of the four plots, Fig. 3.6 shows the best control where the system state trajectories are effectively attracted to the quantized surface and driven to the desired equilibrium point. 


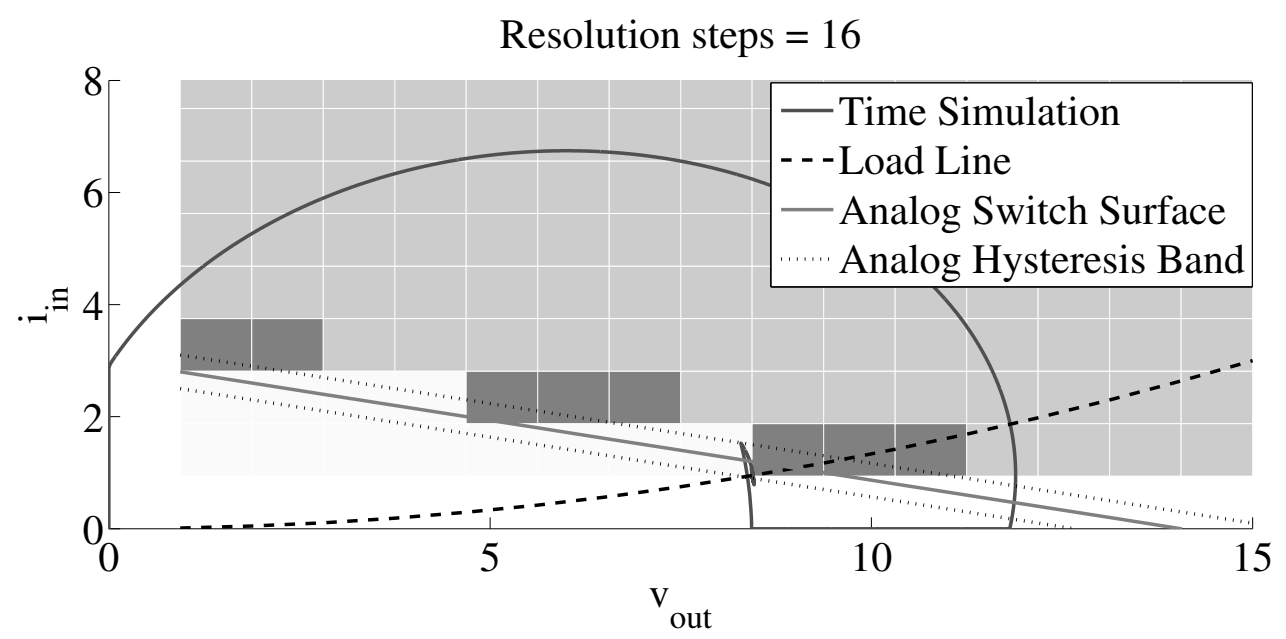

Figure 3.3: State-plane plot for the digital sliding mode control strategy for a sloped reference surface showing the memory resolution effect for a resolution step size of 16 .

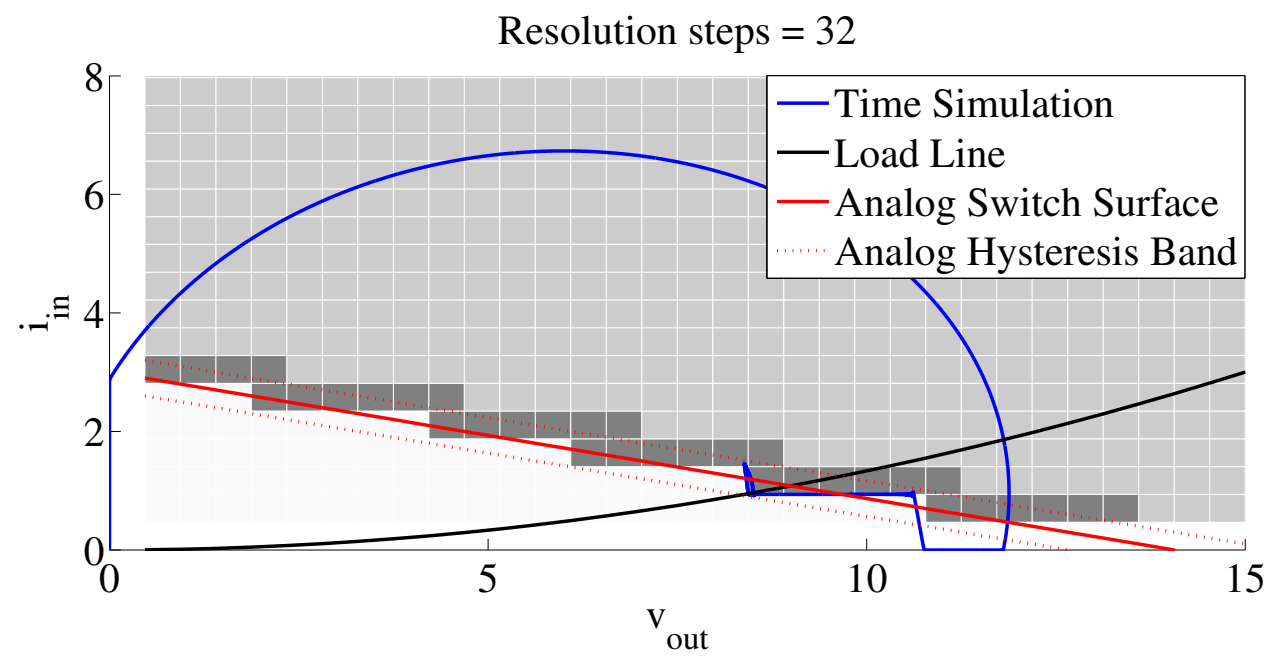

Figure 3.4: State-plane plot for the digital sliding mode control strategy for a sloped reference surface showing the memory resolution effect for a resolution step size of 32 .

The shape of the surface and the operating point depends on the system parameters that may change during operation. Possible changes in the circuit parameters may be taken into 


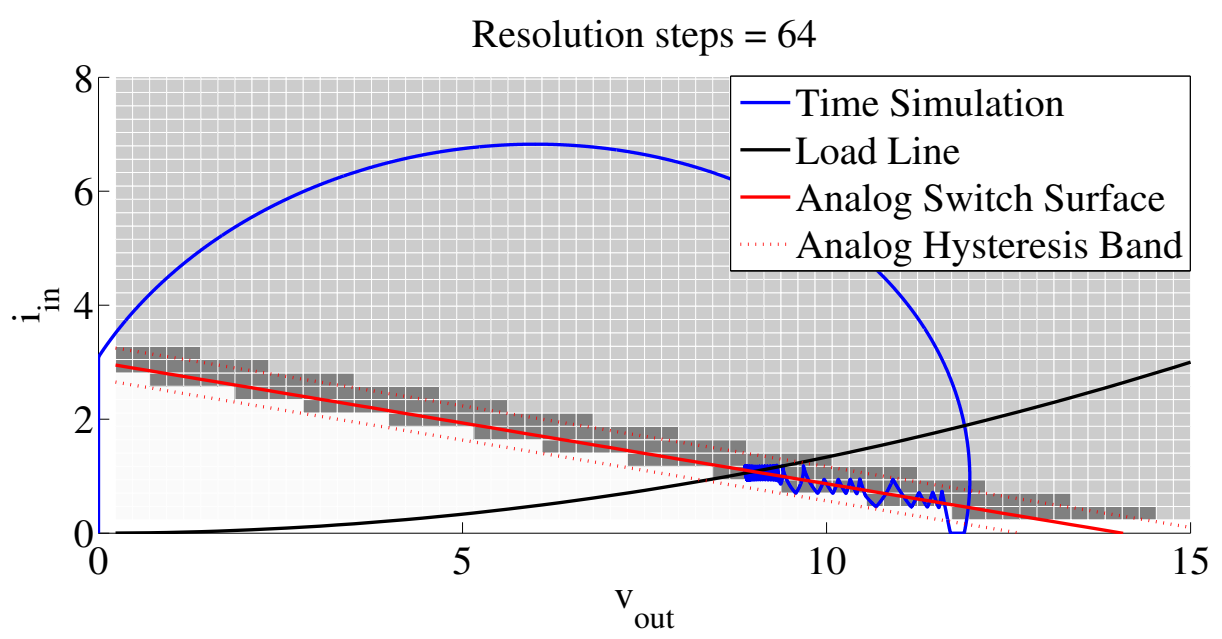

Figure 3.5: State-plane plot for the digital sliding mode control strategy for a sloped reference surface showing the memory resolution effect for a resolution step size of 64 .

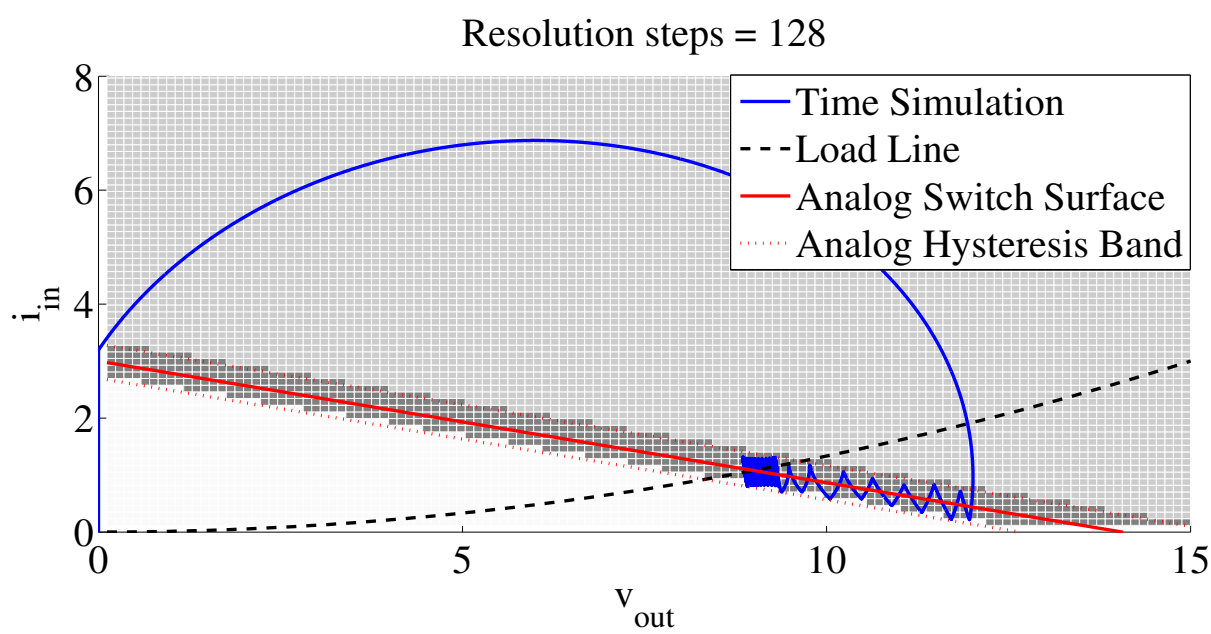

Figure 3.6: State-plane plot for the digital sliding mode control strategy for a sloped reference surface showing the memory resolution effect for a resolution step size of 128 .

account during the process of surface generation in the memory lookup table. The system used for simulation has a $R$ value of $12.5 \Omega$. If there is a step change in the load resistance, the surface and the load line both will change, and hence the equilibrium point will also change. It is necessary to construct this new surface in the memory lookup table. This will 
require additional memory locations to accommodate this additional pixels for this surface and the switch states corresponding to them. The memory lookup table will require to sense the resistance values for the correct lookup for the switch state. Similarly, changes in other system parameters will require more memory locations in the lookup table. It is possible to derive and store many surfaces for many different system parameters $a$-priori and generate the required switch state for the control.

\subsection{Cost comparison}

Implementation of a digital sliding mode control technique in dc-dc boost converters require a complex controller as discussed in Sections 3.2 and 3.3, A high-end digital signal processor or an advanced microcontroller is required to implement complex sliding surfaces in the form of a look-up table in the controller. In this work, the memory table and the control logic is programmed in an EEPROM (ATMEL Part Number AT28C256) and the controller is robust and capable of a fast transient response. A Viertex-5 FPGA platform is used together with dSPACE DS1104 to implement a sliding mode control strategy in a boost converter for fuel cell applications in [92]. However, this is a development tool and hence not included in the price comparison. The work done in [34] used a DSP (TI Part Number TMS320F2812) with a link for computer interface (Code Composer Studio). A 32 bit microcontroller (LPC1768 32 bit ARM Cortex-M3) along with a RTII ethernet connection is used in [93] to implement bang-bang control in dc-dc boost converters. The work in [94] 
used the TMDSCNCD28335 - TMS320F28335 controlCARD from Texas Instruments to implement an adaptive hysteresis band control method in an active power filter application for non sinusoidal supply voltages. As of November 14, 2013, the prices for all the controllers are compared and shown in Table 3.2 [95, 96]. Therefore, the EEPROM that

Table 3.2

Cost comparison between different digital controllers capable of implementing digital sliding mode control strategy in a dc-dc boost converter.

\begin{tabular}{|l|c|}
\hline Controller & Price per unit \\
\hline TMS320F2812 DSP & $\$ 25.65$ \\
\hline $\begin{array}{l}\text { LPC1768 32-bit ARM Cortex-M3 } \\
\text { Microcontroller Unit with RMII Ethernet } \\
\text { connection }\end{array}$ & $\$ 21.25$ \\
\hline $\begin{array}{l}\text { TMDSCNCD28335 - TMS320F28335 } \\
\text { controlCARD }\end{array}$ & $\$ 69.00$ \\
\hline AT28C256 EEPROM & $\$ 8.56$ \\
\hline
\end{tabular}

is used for this work in cheaper than all other controllers capable of performing a similar kind of task. 


\subsection{Simulation and hardware results for implementation of the digital sliding mode control strategy in a dc-dc boost converter}

Three different sliding surfaces are chosen to validate the proposed control strategy. A constant current reference surface is selected at first, which is a straight line in the state-plane diagram [97]. The state-plane diagram has the output voltage state in the $\mathrm{x}$-axis and the input current state along the y-axis. Next, a sloped linear surface in the state-plane diagram is chosen. Finally, a curved non-linear surface is chosen. The non-linear surface is an optimal surface derived for a specific transient condition. The reason for the choice of these surfaces, their derivations and the stability issues are discussed in the following sections.

\subsubsection{Implementation of the digital sliding mode control strategy of a straight line surface}

A constant current reference surface is selected to validate the control strategy. The goal is to drive the input current state of a dc-dc boost converter from a cold start to the surface and stay on it. The dc-dc boost converter acts as a POLC between a dc source and a resistive 
load. Since the goal is to control the input current state only, a single 8 bit ADC is used. For the experimental design, the LSB of the ADC output is not used for noise issues. Effectively 7 output bits of the ADC is interfaced with the EEPROM, thereby yielding a resolution of $38 \mathrm{mV} / \mathrm{bit}$. For this set up, 128 memory locations of the EEPROM are used where the switch states are programmed. The EEPROM reads in the ADC output and generates the switch states as stored in its memory to drive the system state to the desired sliding surface.

In a real-time system, an ADC is used in conjunction with a memory chip. There is an inherent signal propagation delay associated with the use of an ADC, which generates the required hysteresis in the sliding mode controller [84]. Fig. 3.7 illustrates how hysteresis is generated because of a time delay in a signal. An example signal, shown as 'Actual signal' in Fig. 3.7 is delayed by $0.5 \mathrm{~s}$. The delayed signal is shown as 'Time delayed signal' in Fig. 3.7. For a power electronic converter this signal is a state, such as current or voltage. When the time delayed signal reaches the sliding reference surface, according to the switching condition for a pure sliding mode control shown in (3.7), the state trajectory reverses its path.

$$
q= \begin{cases}0 & \text { if } s>0 \\ 1 & \text { if } s<0\end{cases}
$$

But at this point, the actual signal is at a position higher than the reference surface in the time domain plot as shown in Fig. 3.7. Again, when the time delayed signal reaches the 


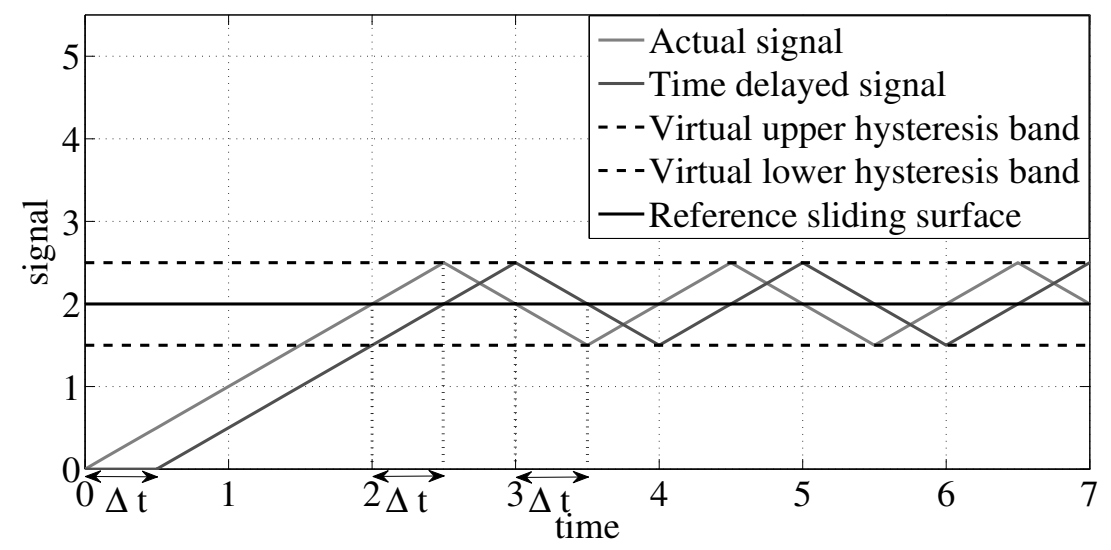

Figure 3.7: Illustration of hysteresis generation due to time delay of a signal.

sliding surface on its return path, the switching takes place according to (3.7), and the state trajectory reverses its path. This time the actual signal is at a position lower than the reference surface in the time domain plot. This creates an upper and a lower band around the reference surface, where the actual signal changes its path at every switching instant. These bands are called the hysteresis bands between which the switching occurs. Therefore, a time delay in the signal can generate the hysteresis required in a sliding mode controller. This ADC latency simplifies the hardware complications by reducing the number of bits necessary in the memory look-up table. There is no need to use additional memory locations for hysteresis generation. The ADC latency takes care of this and the delay generates the required hysteresis for the sliding-mode controller. The analysis and impact of ADC latency on hysteresis is shown in the following sections. 


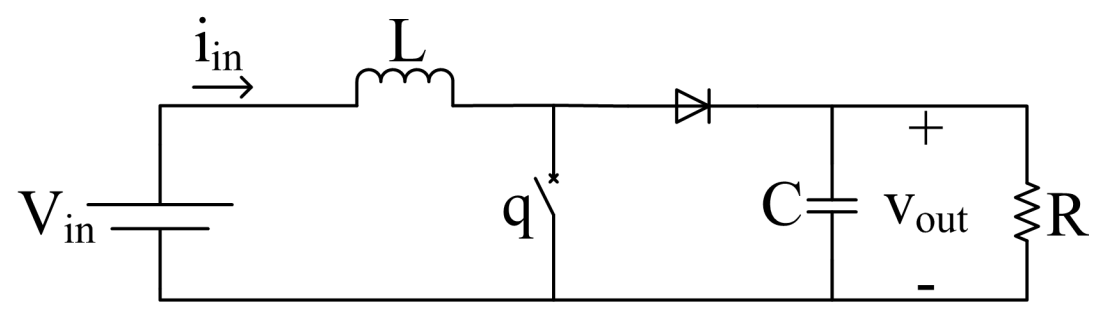

Figure 3.8: Dc-dc boost converter with resistive load.

\subsubsection{Stability analysis for the straight line surface}

For the states of a system to follow a particular sliding surface, the state trajectories need to be attracted towards the manifold. The sliding surface, therefore, needs to be a stable one for the system under consideration. It is therefore necessary to prove that the straight line surface chosen for a dc-dc boost converter is a stable surface and the state trajectories are attracted to it.

The dynamic equations for a dc-dc boost converter as shown in Fig. 3.8 are shown in (3.1). The switch state for the dc-dc boost converter is determined by the sliding control law

$$
s=f(x)=0
$$

where $x$ represents the converter states and $f(x)$ is the deviation of the states from the derived geometric surface. The switching control law for the dc-dc boost converter is 
shown in (3.7). For a system to follow a particular sliding surface, the state trajectories are attracted towards the manifold when

$$
s \dot{s}<0 \text {. }
$$

Equation (3.9) is the required condition for stability. If (3.9) is satisfied for a region of the state space that contains the sliding surface $(\sqrt{3.8})$, then the state trajectories that leave any point on this region will converge to the sliding surface and once they reach it, they will stay on it [75]. The straight line surface chosen is defined as

$$
s=i_{\text {in }}-i_{\text {inref }}=0 \text {. }
$$

The derivative of the surface is given by,

$$
\dot{s}=\dot{i}_{\text {in }}=\frac{V_{\text {in }}}{L}-\frac{(1-q) v_{\text {out }}}{L} .
$$

Using (3.7) in (3.11), for the region $s<0$,

$$
\dot{s}=\frac{V_{i n}}{L}
$$

For a dc-dc boost converter, and for any power converters in general, the input voltage and the inductance is always positive. For the example system shown in Table 3.3, the R.H.S. 
of (3.12) is positive. Therefore, for the region $s<0$,

$$
s \dot{s}<0 .
$$

For the region $s>0$,

$$
\dot{s}=\frac{V_{\text {in }}}{L}-\frac{v_{\text {out }}}{L} .
$$

In the region $s>0$, for stability, $v_{\text {out }}>V_{\text {in }}$, since $L$ is always positive. This is the required condition for stability. The example dc-dc boost converter system is given a cold start. The states are at $(0,0)$ and lie in the region $s<0$. It has already been proved that all the states lying in the region $s<0$ will slide to the surface. As an illustration, Fig. 3.9 shows the phase portrait for the example dc-dc boost converter for the region of operation of the system. The phase portrait shows that all the state trajectories are attracted towards the surface and if any of them leave the surface, it again slides back to the surface obeying the switching control law shown in (3.7). Therefore it may be concluded that the sliding surface under consideration for this dc-dc boost converter system is stable and the states are attracted to the surface. 


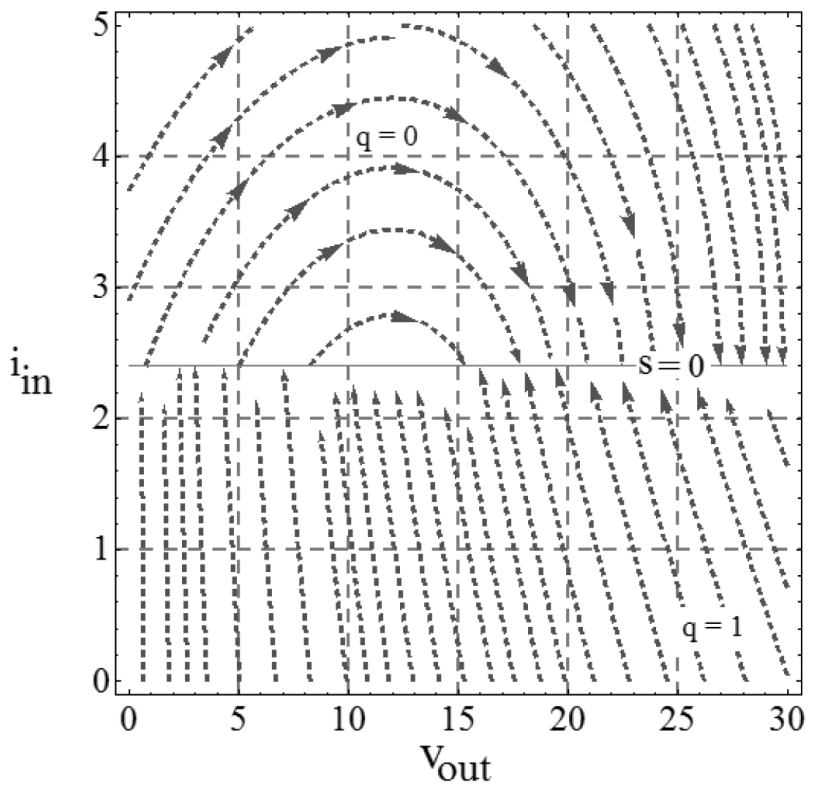

Figure 3.9: Phase portrait of dc-dc boost converter states for the straight line reference sliding surface.

\subsubsection{Simulation results for the straight line reference surface}

A simulation for the system described above is carried out in MATLAB/Simulink. The system parameters selected are shown in Table 3.3 , The objective is to drive the input current state trajectory to the constant current reference surface. The final desired input current value is $2.4 \mathrm{~A}$. The ADC converter delay is $2.5 \mu \mathrm{s}$, which generates the hysteresis required by the controller. The simulation results are shown in Fig. 3.10 to Fig. 3.11, Fig.

Table 3.3

Example dc microgrid parameters for a straight line surface control.

\begin{tabular}{|c|c|c|c|c|c|c|}
\hline$V_{\text {in }}$ & $\mathrm{L}$ & $\mathrm{C}$ & $R_{\text {load }}$ & ADC resolution & ADC delay & Memory locations \\
\hline $12 \mathrm{~V}$ & $1 \mathrm{mH}$ & $100 \mu \mathrm{F}$ & $12.5 \Omega$ & $38 \mathrm{mV} / \mathrm{bit}$ & $2.5 \mu \mathrm{s}$ & 128 \\
\hline
\end{tabular}




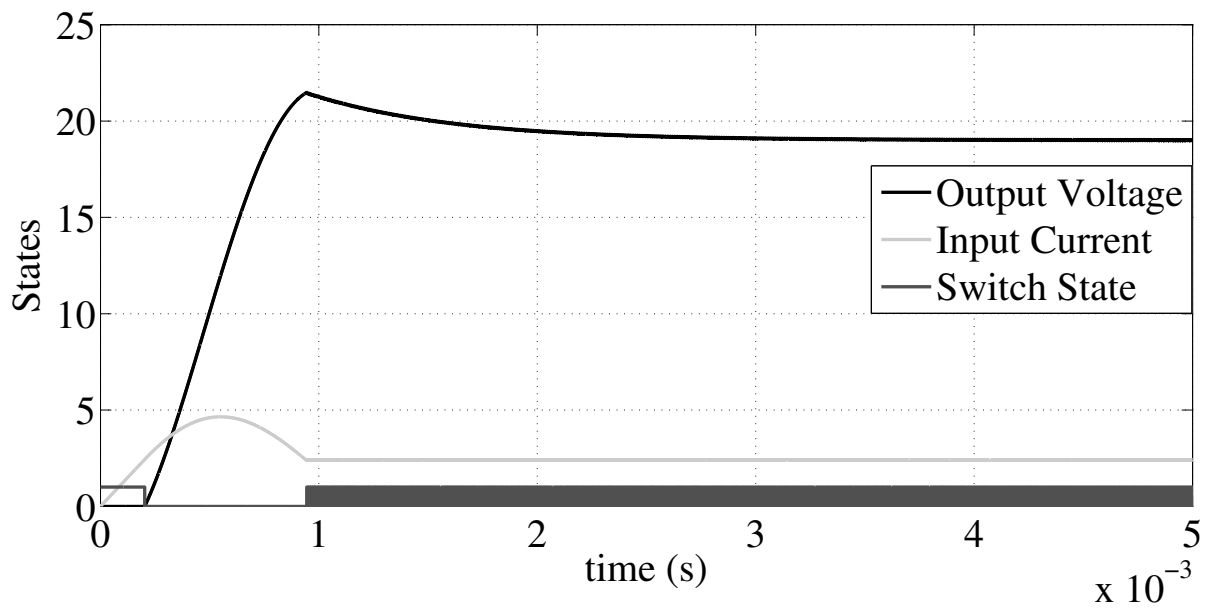

Figure 3.10: Time domain plot for the digital sliding mode control strategy for a straight line surface in a dc-dc boost converter.

3.10 shows the variation of the input current and output voltage states with time. It may be noted that the input current reaches a value of $2.4 \mathrm{~A}$ from a cold start thereby validating the control strategy. Fig. 3.11 shows the state-plane plot of the output voltage and input current states along with the reference sliding surface. The control strategy drives the state trajectories to the surface and compels the states to stay on the surface.

\subsubsection{Experimental results for the straight line reference surface}

An experimental hardware set up was done for the same system parameters as in Table 3.3 . The hardware set up is shown in Fig. 3.12. The straight line surface is programmed into 


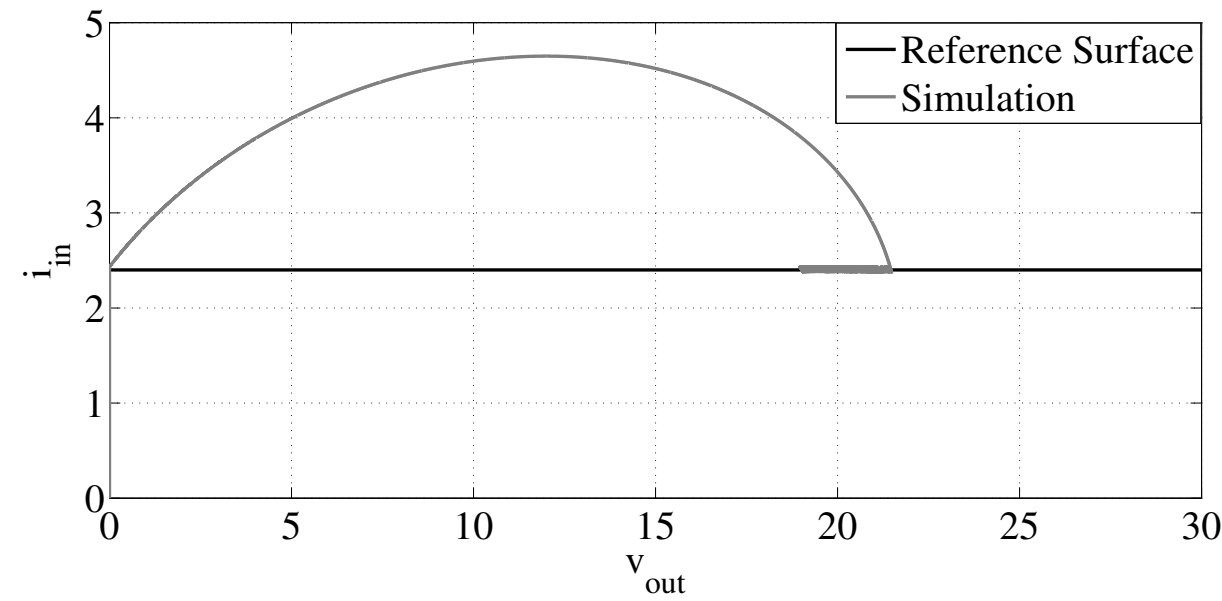

Figure 3.11: State-plane plot for the digital sliding mode control strategy for a straight line surface in a dc-dc boost converter.

128 memory locations of the EEPROM (Part Number AT28C256) and the ADC is set for a propagation delay of $2.5 \mu \mathrm{s}$. The dc-dc boost converter is constructed in a flexible power electronics drive board. The switch signal is given to one of the phases of the inverter circuit and the load is connected across another phase of the inverter. Fig. 3.13 shows the variation of the input current, output voltage and switch states with time. It may be noted that the input current reaches a value of $2.4 \mathrm{~A}$ from a cold start thereby experimentally validating the control strategy. Fig. 3.14 shows the state trajectories in the steady state, indicating that they have reached the reference surface at a value of $2.4 \mathrm{~A}$ and they stay on it. Hence, the straight line surface is a stable surface and is effectively used as a reference surface. The digital sliding mode hysteretic controller could be designed effectively such that the states slide along the reference surface. 


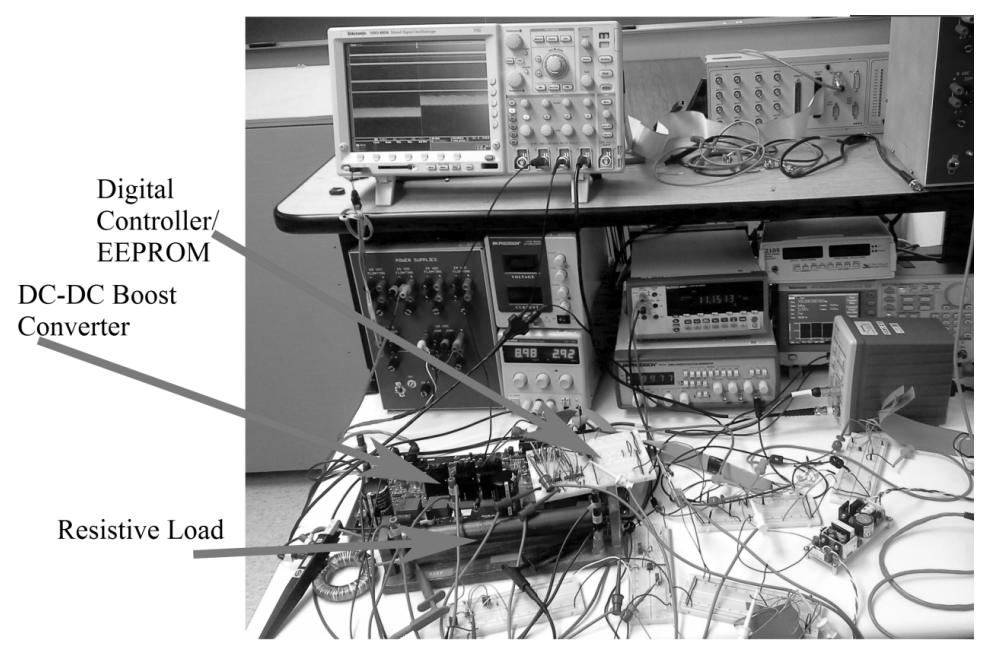

Figure 3.12: Experimental hardware set up for the digital hysteretic sliding mode control implementation in a dc-dc boost converter.

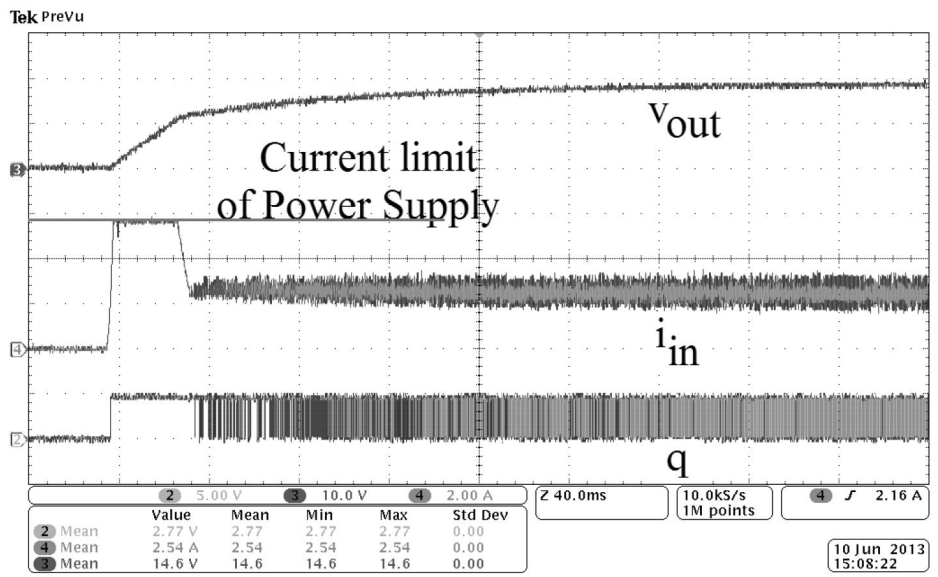

Figure 3.13: Experimental time domain plot for the digital sliding mode control strategy for a straight line surface in a dc-dc boost converter. 


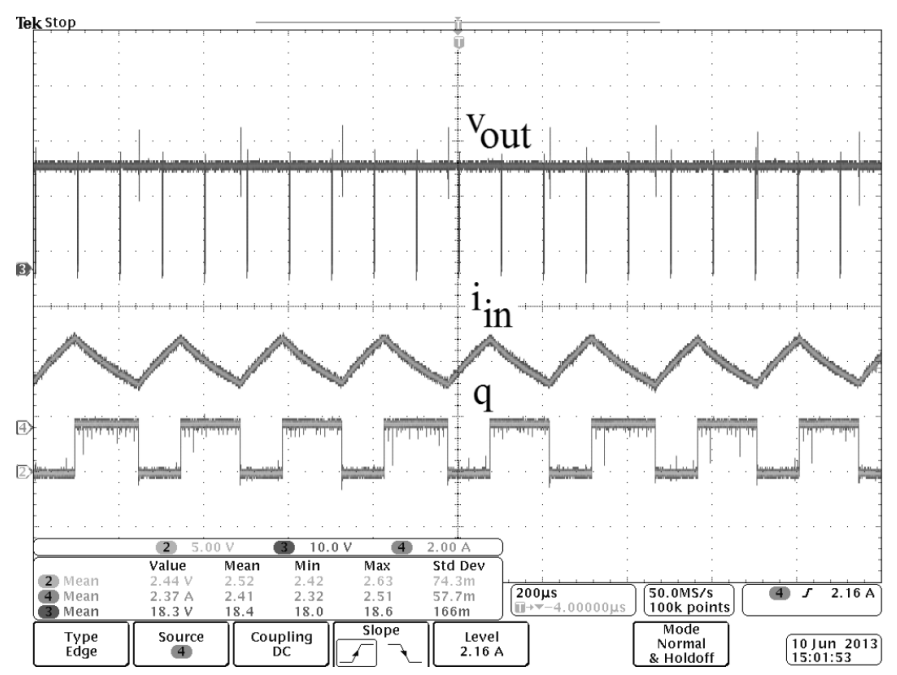

Figure 3.14: Experimental time domain plot in the steady state for the digital sliding mode control strategy for a straight line surface in a dc-dc boost converter.

\subsubsection{Analysis of the ADC latency for the straight line reference surface}

The simulation and experimental results in the previous subsection confirms that no additional hardware set-up is necessary to generate the required hysteresis band in the digital controller. Instead, the ADC latency is capable of generating this required hysteresis [84]. If the delay time is increased, the hysteresis will increase and vice versa, as shown in Fig. 3.7. Hence, higher the latency, greater the hysteresis band and vice versa. The ADC converter used for the experimental set up has three signal propagation delay settings. The default setting is $2.5 \mu \mathrm{s}$., and two extreme settings of $2.2 \mu \mathrm{s}$. and $2.8 \mu \mathrm{s}$. Fig. 3.15 shows the hysteresis generated due to the default setting of $2.5 \mu \mathrm{s}$. conversion delay. Fig. 3.16 shows the experimental results for a smaller delay of $2.2 \mu s$. and Fig. 3.17 shows the results for a greater delay of $2.8 \mu \mathrm{s}$. It may be noted from the results that greater the delay, 
larger the hysteresis band and vice versa.

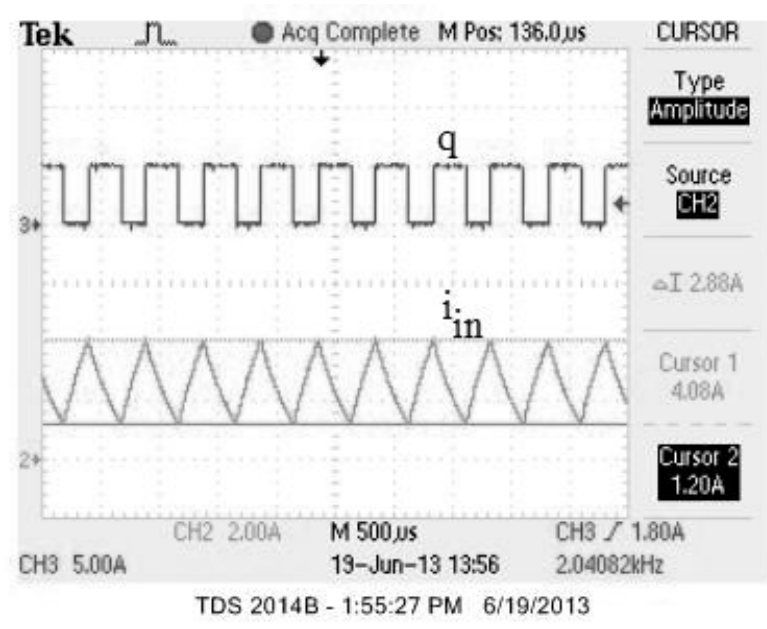

Figure 3.15: ADC delay of $2.5 \mu$ s generating a hysteresis band of $2.88 \mathrm{~A}$ for the digital sliding mode control strategy for a straight line surface in a dc-dc boost converter.

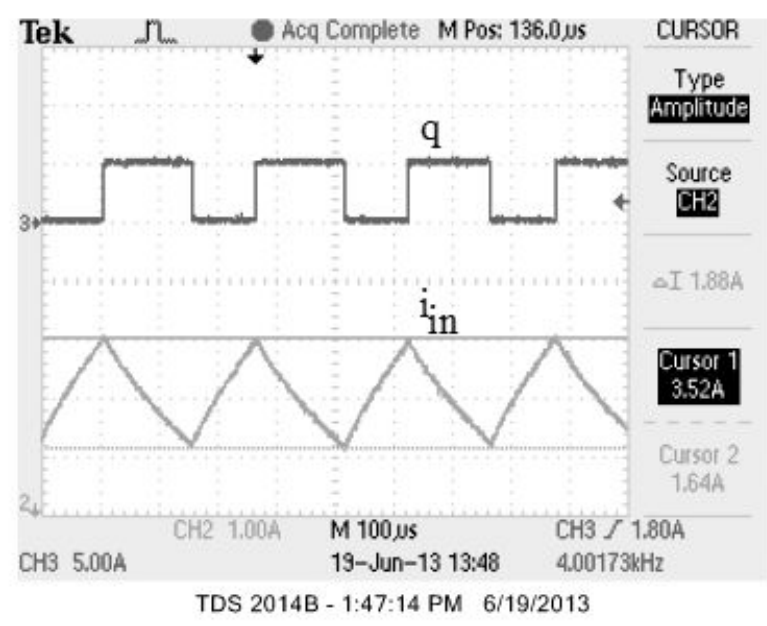

Figure 3.16: ADC delay of $2.2 \mu$ s generating a hysteresis band of $1.88 \mathrm{~A}$ for the digital sliding mode control strategy for a straight line surface in a dc-dc boost converter. 


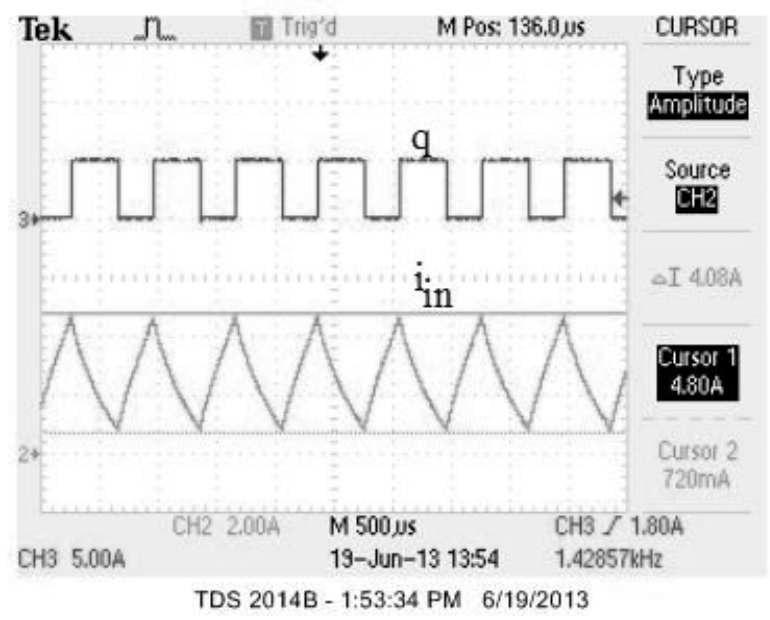

Figure 3.17: ADC delay of $2.8 \mu$ s generating a hysteresis band of $4.08 \mathrm{~A}$ for the digital sliding mode control strategy for a straight line surface in a dc-dc boost converter.

However, there are both upper and lower bounds to the delay in the ADC converter, beyond which this strategy will cease to work. If the delay is made very small, the hysteresis band will become extremely small and result to very high switching frequency. On the other hand, if the delay is very big, then the memory table control logic will react to a very delayed signal and output an erroneous switch state.

\subsubsection{Linear sloped surface}

A linear sloped surface shown in Fig. 3.18 is next chosen to further validate the proposed control strategy. Any practical reference surface is a non-linear surface in the state-plane diagram. However, a number of sloped linear approximations to any complex non-linear 
surface may be made for real-time implementation. The choice of a sloped straight line reference surface is therefore pertinent here. The controller designed for this type of a sloped reference surface is a guideline for any type of linear reference surface. The slope and the y-intercept depends on the system design specifications. The sloped line in the state-plane plot is given by

$$
i_{\text {in }}=m+b v_{\text {out }} \text {. }
$$

The slope of the line is determined by (3.6). The load line for a dc-dc boost converter is given by (3.4). Based on the values shown in Table 3.1, the desired equilibrium point in the state-plane plot is $(9 \mathrm{~V}, 1.08 \mathrm{~A})$. In this type of a controller, the aim is to drive the input current and the output voltage states of the dc-dc boost converter from a cold start to reach the equilibrium point on the surface and stay on it. The equilibrium point is defined by the intersection of the load line and the surface. Since the goal here is to control both the input current and output voltage states, two 8 bit ADCs are used. The output voltage and input current states are scaled down before sampling. In this set up also, the LSB of the ADC converter output is not used for noise issues. Effectively 7 output bits of each of the ADC converters are interfaced with the EEPROM, thereby yielding a resolution of 38 $\mathrm{mV} / \mathrm{bit}$. The resolution for the output voltage is $0.2 \mathrm{~V}$ and that of the input current is 38 $\mathrm{mA}$. For this set up, $2^{14}=16384$ memory locations of the EEPROM are used where the switch states are programmed. The EEPROM reads in the ADC output and generates the switch states as stored in its memory to drive the system state to the desired equilibrium 


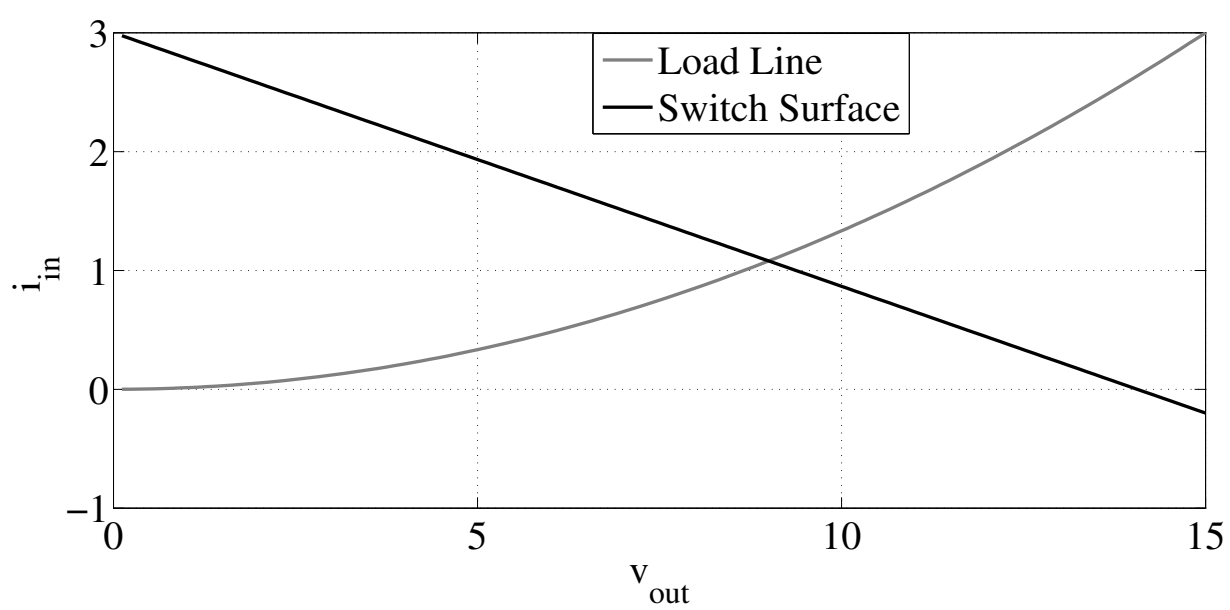

Figure 3.18: Sloped surface in the state-plane plot for the digital sliding mode control strategy in a dc-dc boost converter.

point on the sliding surface.

\subsubsection{Stability analysis for the linear sloped surface}

The linear approximation of any non-linear surface generates a sloped surface in the state-plane. The sloped linear sliding surface needs to be a stable one for the system under consideration. It is therefore necessary to prove that the sloped linear surface chosen for a dc-dc boost converter is a stable surface. This derivation will generate the condition of stability for any sloped linear surface and then show that the sloped surface for the example system considered is stable.

The dynamic equations for a dc-dc boost converter is shown in (3.1). The switching control 
law for the dc-dc boost converter is shown in (3.7). Equation (3.9) is the required condition for stability. A sloped linear surface is shown in (3.5). The derivative of the surface is given by,

$$
\dot{s}=\dot{i}_{\text {in }}-b \dot{v}_{\text {out }}=\frac{V_{\text {in }}}{L}-\frac{(1-q) v_{\text {out }}}{L}-\frac{b(1-q) \dot{i}_{\text {in }}}{C}+\frac{b v_{\text {out }}}{C R} .
$$

Using (3.7) in (3.16), for the region $s<0$,

$$
\dot{s}=\frac{V_{\text {in }}}{L}+\frac{b v_{\text {out }}}{C R} .
$$

Therefore, for the region $s<0, \dot{s}>0$ when

$$
\frac{V_{\text {in }}}{L}>-\frac{b v_{\text {out }}}{C R}
$$

which is the required stability criteria for this region. For the region $s>0$,

$$
\dot{s}=\frac{V_{\text {in }}}{L}-\frac{v_{\text {out }}}{L}-\frac{b i_{\text {in }}}{C}+\frac{b v_{\text {out }}}{C R} .
$$

For the region $s>0, \dot{s}<0$ when

$$
\frac{V_{\text {in }}}{L}<\frac{C R v_{\text {out }}-L b v_{\text {out }}}{L C R}+\frac{b i_{\text {in }}}{C} .
$$

For the example dc-dc boost converter considered for this analysis, the system parameters 
are shown in Table 3.1. Using these parameters, for $s<0$, (3.17) reduces to

$$
\dot{s}=6000-170.4 v_{\text {out }} \text {. }
$$

For $\dot{s}>0$, the maximum value that the output voltage can take is $35.2 \mathrm{~V}$. Fig. 3.18 shows that for the region $s<0$, the maximum value of the output voltage is $14 \mathrm{~V}$. Therefore, for the example system, $\dot{s}>0$ for the region $s<0$. Hence, the product is always $s \dot{s}<0$. For the region $s>0$, 3.19) reduces to

$$
\dot{s}=2130\left(\frac{v_{\text {out }}}{R}-i_{\text {in }}\right)+1000\left(V_{\text {in }}-v_{\text {out }}\right) .
$$

For the R.H.S. of (3.22) to be negative, in the region $s>0, v_{\text {out }}>V_{\text {in }}$ and $i_{\text {in }}>\frac{v_{\text {out }}}{R}$. This is the required stability criteria. The example dc-dc boost converter system is given a cold start. Both the states are at $(0,0)$ at this point and belong to the region $s<0$. It has already been proved that $\dot{s}>0$ for the region $s<0$ for the example system. Therefore, the states will reach the surface from a cold start. The phase portrait for the example dc-dc boost converter for a region around the sliding surface is shown in Fig. 3.19. The phase portrait shows that if the states leave the surface, it again slides back to the surface obeying the switching control law shown in (3.7). Therefore it may be concluded that the sliding surface under consideration for this dc-dc boost converter system is stable and the states are attracted to the surface. 


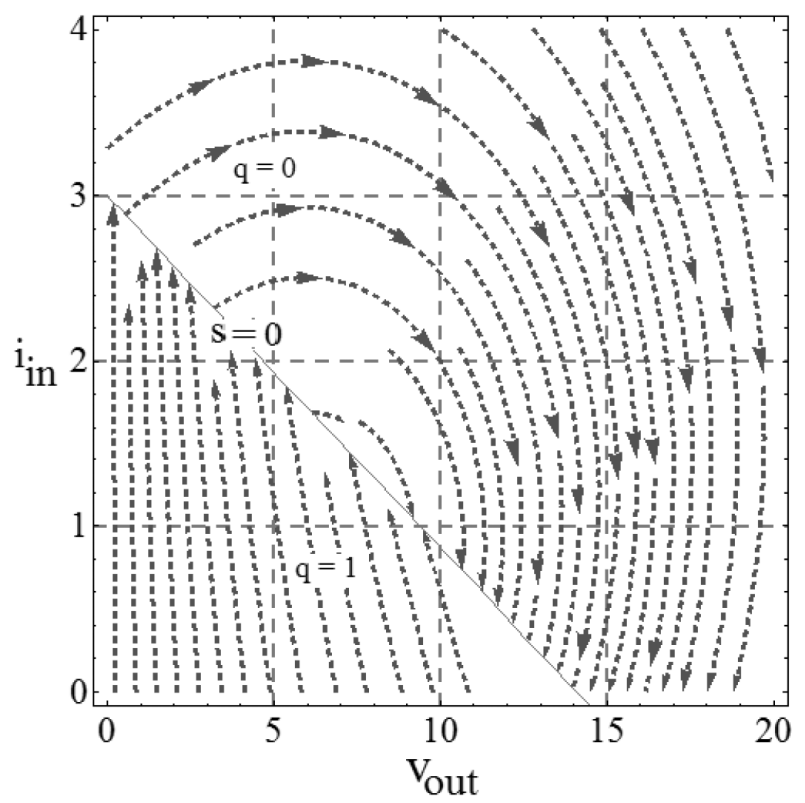

Figure 3.19: Phase portrait of dc-dc boost converter states for the sloped reference sliding surface.

\subsubsection{Simulation results for the sloped reference surface}

A simulation for the system with sloped reference surface is done in MATLAB/Simulink. The system parameters selected are shown in Table 3.1. The objective is to drive the input current and output voltage state trajectories to the equilibrium point of the surface shown in Fig. 3.18, The ADC converter delay is $2.5 \mu s$, its default value, which generates the hysteresis required by the controller.

The simulation results are shown in Fig. 3.20 and Fig. 3.21 Fig. 3.20 shows the 

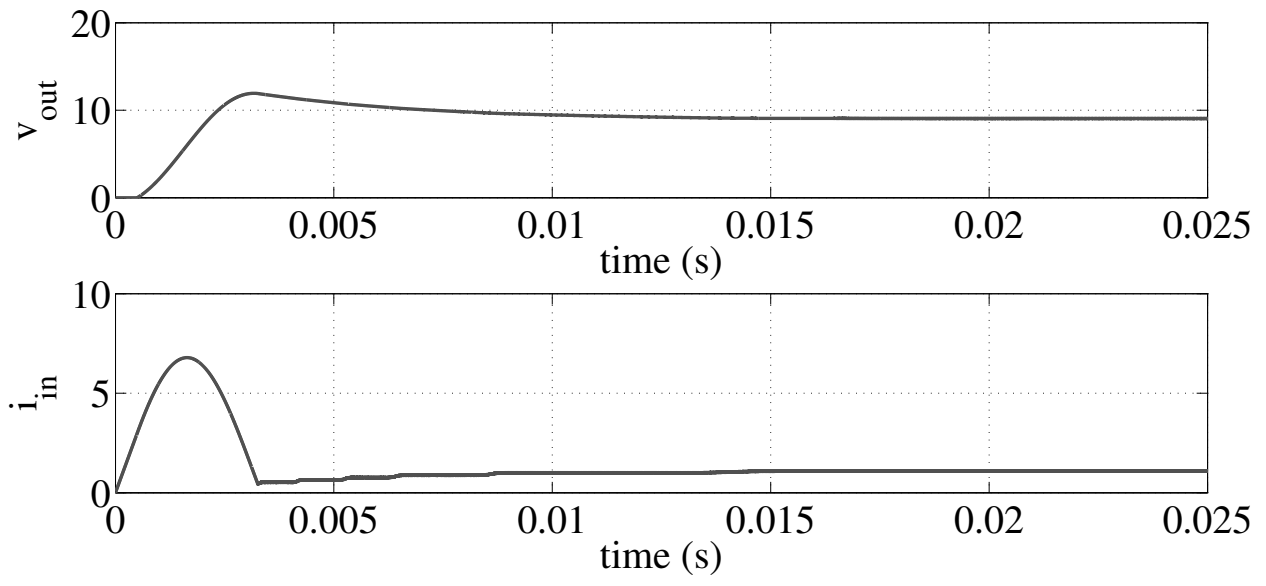

Figure 3.20: Time domain plot for the digital sliding mode control strategy for a sloped reference surface in a dc-dc boost converter.

variation of the input current and output voltage states with time. It may be noted that the input current and output voltage states reach the desired equilibrium point from a cold start thereby validating the control strategy. Fig. 3.21 shows the state-plane plot of the output voltage and input current states along with the reference sliding surface. The control strategy drives the state trajectories to the surface, compels the states to stay on the surface and drives them to the desired equilibrium point. 


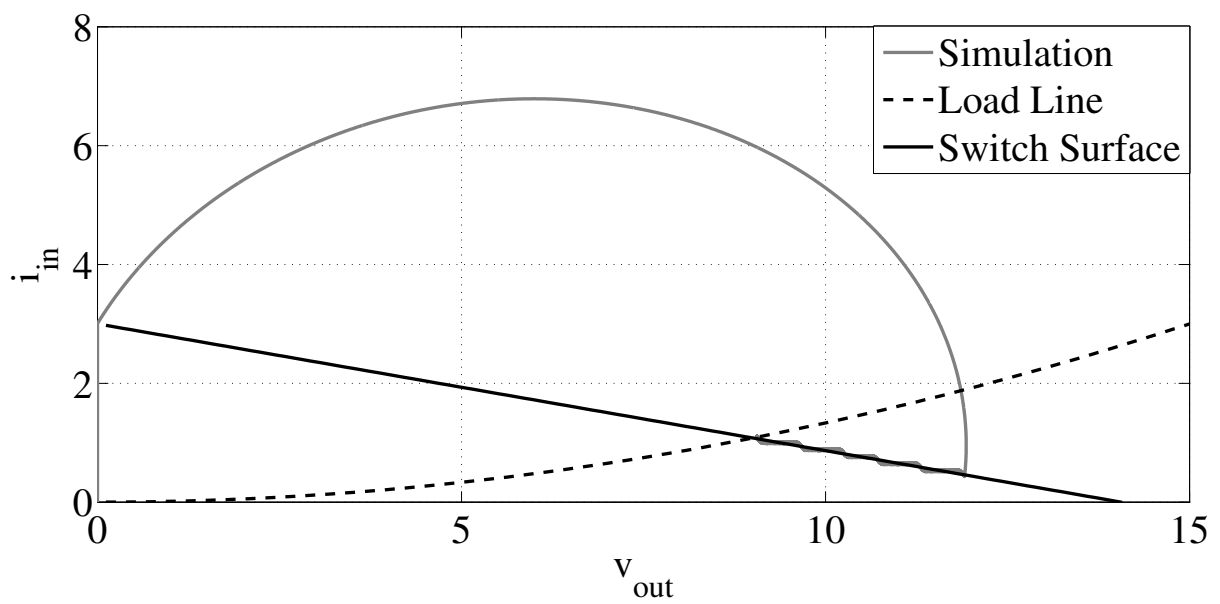

Figure 3.21: State-plane plot for the digital sliding mode control strategy for a sloped reference surface in a dc-dc boost converter.

\subsubsection{Experimental results for the sloped reference surface}

The system parameters in Table 3.1 are used in an experimental set-up to validate the control strategy in a real-time hardware. The sloped surface shown in Fig. 3.18 is programmed into 16384 memory locations of the EEPROM and the ADC converter is set for a propagation delay of $2.5 \mu \mathrm{s}$. In this set up, 14 input pins of the EEPROM are used to accommodate the signals coming from the two ADCs. The boost converter and the load set up are similar to that of the straight line surface test case. Fig. 3.22 shows the variation of the input current, output voltage and switch states with time. It may be noted that the output voltage finally reaches a value of around $9 \mathrm{~V}$ and the input current reaches an average value of around 1.20 A from a cold start, which is close to the desired equilibrium point values, thereby experimentally validating the control strategy. Fig. 3.23 shows the state trajectories in the steady state, indicating that they have reached values close to the equilibrium point. 


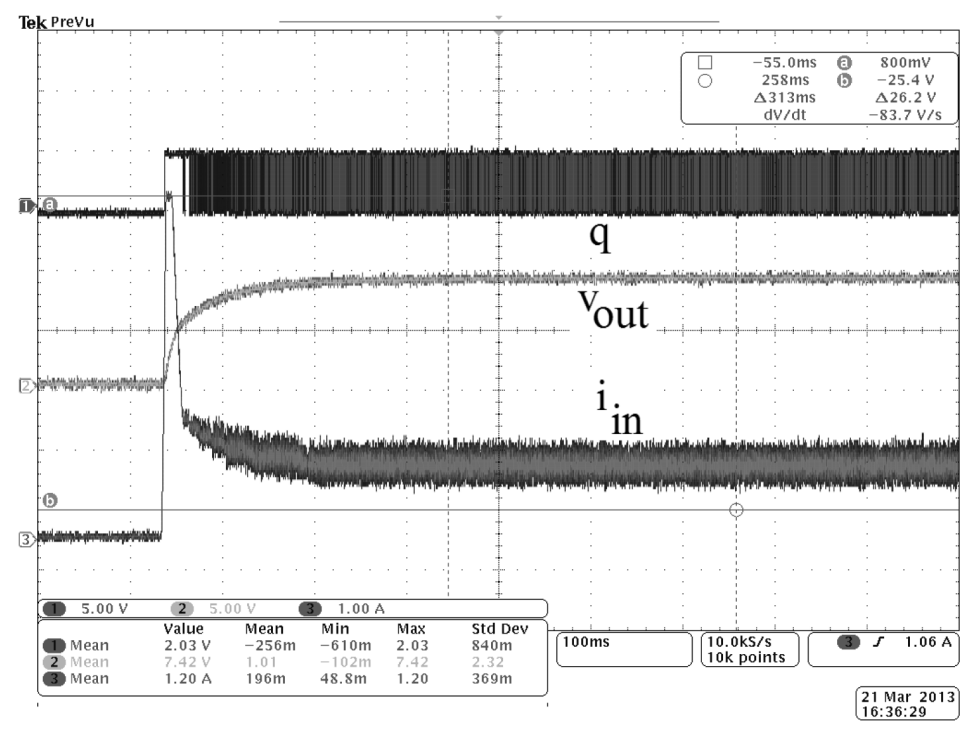

Figure 3.22: Experimental time domain plot for the digital sliding mode control strategy for a sloped reference surface in a dc-dc boost converter.

Fig. 3.24 shows the state-plane plot obtained from the experimental data. This figure shows that the controller drives the system state trajectories to the required sliding surface and to the equilibrium point. The deviation from the simulation results may be attributed to the various losses in the hardware, signal delays other than the ADC converters in the circuit and the ADC converter output resolution. Also, in the simulation, a lossy converter model was not considered. In Fig. 3.22, it may be observed that the input current trajectory never crosses a value of $5.8 \mathrm{~A}$. This is because the current limit of the power supply is set to be 5.8 A. Hence, the sloped surface can be successfully implemented as a reference surface in the memory chip and the digital sliding mode hysteretic controller could be designed effectively such that the states slide along the reference surface and reach the desired equilibrium point. 


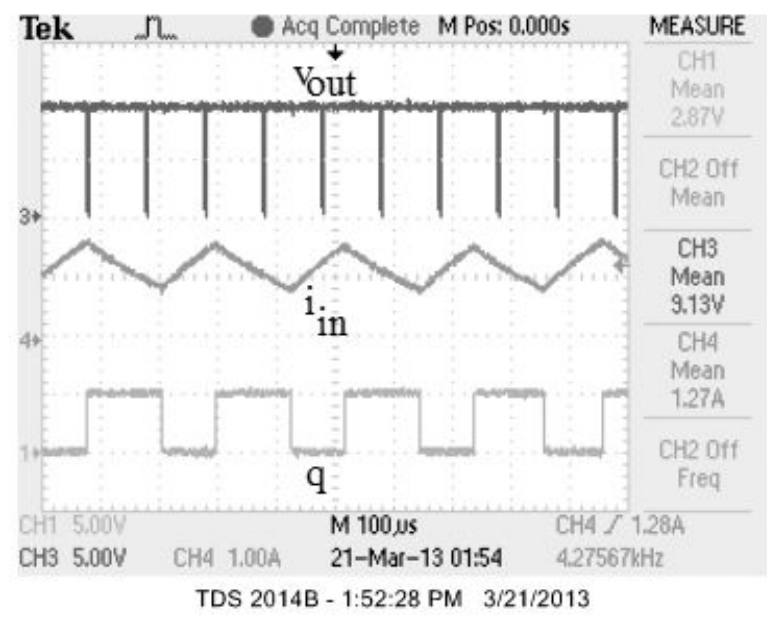

Figure 3.23: Experimental time domain plot in the steady state for the digital sliding mode control strategy for a sloped reference surface in a dc-dc boost converter.

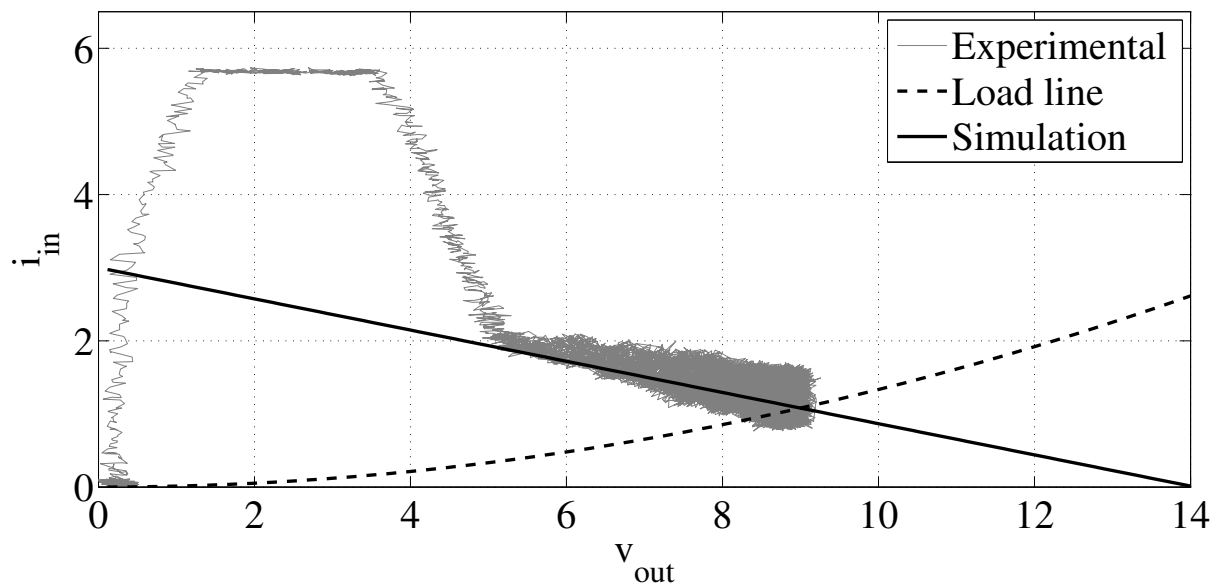

Figure 3.24: Experimental state-plane plot for the digital sliding mode control strategy for a sloped reference surface in a dc-dc boost converter. 


\subsubsection{Optimal surface}

The control strategy has been validated for cold starts with straight line and sloped reference surfaces. Next a non-linear optimal surface as shown in Fig. 3.25 is chosen to validate the proposed control strategy for a step change in load. An important consideration of any dc-dc converter design is to keep its terminal voltage constant. Here, the aim is to make the input current and the output voltage states of the dc-dc boost converter follow the surface and reach the new equilibrium point corresponding to the changed load condition. The output voltage and input current states are scaled down before sampling. In this set up also, the LSB of the ADC output is not used for noise issues. Effectively 7 output bits of each of the ADCs are interfaced with the EEPROM, thereby yielding a resolution of $38 \mathrm{mV} / \mathrm{bit}$. The resolution for the output voltage is $0.4 \mathrm{~V}$ and that of the input current is $80 \mathrm{~mA}$. An additional input for the EEPROM becomes necessary to notify the EEPROM of the load condition of the system. A step change in the load is sensed via this additional input of the EEPROM and it generates the switch states accordingly. The chip program is done taking the load change into consideration. The EEPROM reads in the ADC converter output and generates the switch states as stored in its memory to drive the system state to the desired equilibrium point on the optimal sliding surface. 


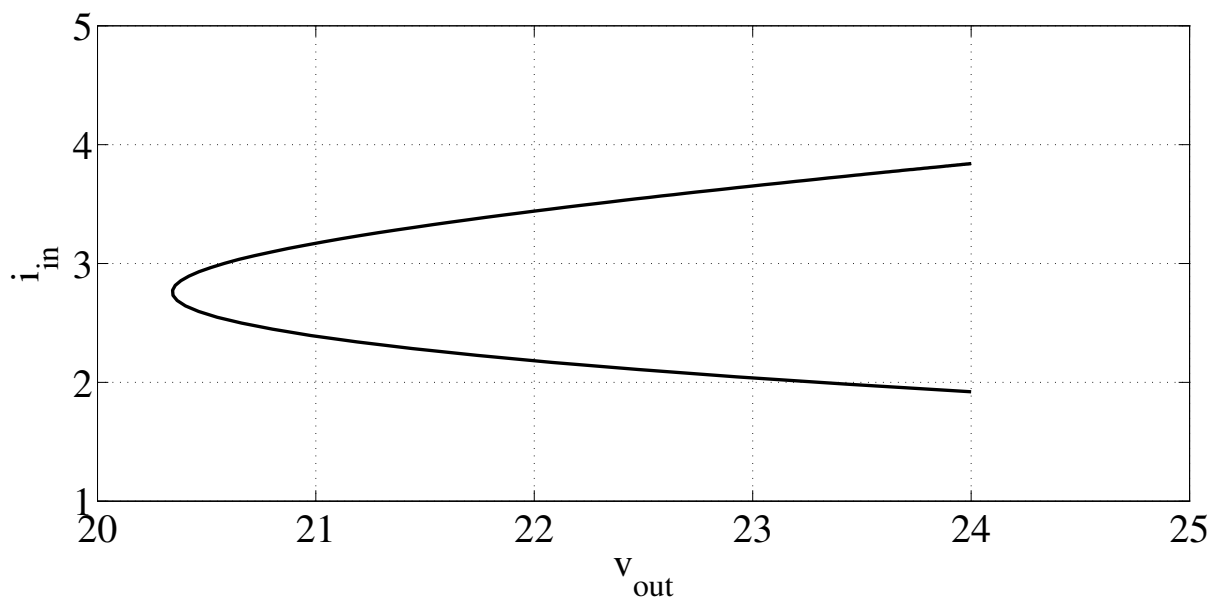

Figure 3.25: Optimal surface in the state-plane plot for the digital sliding mode control strategy in a dc-dc boost converter.

\subsubsection{Derivation of the optimal reference surface}

For the system in Fig. 3.1, an energy balance model [1], as shown in (2.1) is used to derive the optimal surface. For the derivation, stored energy and input power of the POLC are chosen as the state variables. During a transient condition, such as a step change in the load, the objective of the control is to maintain the terminal characteristics of the system at nominal values. For the system shown in Fig. 3.1, the objective is to regulate the output voltage of the POLC. According to (2.3), regulation of the output voltage implies conservation of the energy stored in the POLC. The set of dynamic equations for the system is given as

$$
\begin{aligned}
& \dot{w}=p_{\text {in }}-\frac{2 w}{C R} \\
& \dot{p}_{\text {in }}=u .
\end{aligned}
$$


The rate of change of the power inflow is the control $u$ as shown in (3.23).

To derive the optimal state trajectories of the system, an objective function of the general form

$$
J=\int_{0}^{t_{f}} g(x) d t
$$

is chosen [86]. The choice of the path objective is

$$
g(x)=\left(w-w_{\text {nom }}\right)^{2}+u^{2}
$$

$w_{\text {nom }}$ is the nominal values of the energy functions. The path objective (3.25) seeks to keep the energy as close to nominal values as possible and the rate of change of the power flow functions are minimized. During a transient condition the objective of the control is to make the state trajectories travel from the initial operating conditions to the new steady-state conditions based on the minimization of the objective function (3.24). The initial conditions for the system are

$$
\begin{aligned}
& w(0)=w_{\text {nom }} \\
& p_{\text {in }}(0)=p_{\text {ino }} .
\end{aligned}
$$

The problem now consists of the state equations (3.23), the objective function (3.24) and the initial conditions (3.26). For the solution of the optimal control problem, Pontryagin's 
minimum principle [18, 86] is used. This minimum principle is capable of providing only open loop solutions [86]. However, the open loop solutions of various a-priori events will be used to form a control manifold as the basis of a real-time feedback control algorithm. The Hamiltonian for the path minimization is

$$
H=w^{2}+u^{2}+\lambda_{1}\left(p_{i n}-\frac{2 w}{C R}\right)+\lambda_{2} u
$$

where $\lambda_{1}$ and $\lambda_{2}$ are the co-states [86]. The solution for the controls $u^{*}$ is found from

$$
\frac{d H}{d u}=0
$$

and the co-states are found from

$$
\begin{aligned}
& \dot{\lambda_{1}}=-\frac{d H}{d w} \\
& \dot{\lambda}_{2}=-\frac{d H}{d\left(p_{\text {in }}\right)} .
\end{aligned}
$$

Thus the augmented set of equations are

$$
\begin{aligned}
& \dot{w}=p_{\text {in }}-\frac{2 w}{C R} \\
& \dot{p}_{\text {in }}=u^{*} \\
& \dot{\lambda}_{1}=-\frac{d H}{d w} \\
& \dot{\lambda}_{2}=-\frac{d H}{d\left(p_{\text {in }}\right)} .
\end{aligned}
$$


The boundary conditions are

$$
\begin{aligned}
& w(0)=w_{\text {nom }} \\
& p_{\text {in }}(0)=p_{\text {ino }} \\
& \lambda_{1}\left(t_{f}\right)=0 \\
& \lambda_{2}\left(t_{f}\right)=0 .
\end{aligned}
$$

This is a two-point boundary value problem (BVP). For an optimal solution, the Hamiltonian is a minimized scalar value. The solution of (3.30) with the boundary conditions (3.31) gives the optimal trajectory for the stored energy $w$ and the power flow $p_{\text {in }}$ for a step change in the load. To be implemented in real-time hardware, this trajectory needs to be mapped to voltage and current states. The conversion equation is

$$
\begin{aligned}
& v_{\text {out }}=\sqrt{\frac{2 w}{C}} \\
& i_{\text {in }}=\frac{p_{\text {in }}}{V_{\text {in }}}
\end{aligned}
$$

For the system shown in Table 3.4 and Table 3.5, the optimal control problem is solved and the reference optimal trajectory for this specific case of load change is shown in Fig. 3.25.

\section{Table 3.4}

Initial system parameters for the optimal reference surface control in a dc-dc boost converter.

\begin{tabular}{|c|c|c|c|c|c|c|c|c|}
\hline$V_{\text {in }}$ & $\mathrm{L}$ & $\mathrm{C}$ & ADC resolution & ADC delay & Chip Memory & $v_{\text {out }}$ & $i_{\text {in }}$ & $R_{\text {load }}$ \\
\hline $12 \mathrm{~V}$ & $1 \mathrm{mH}$ & $100 \mu \mathrm{F}$ & $38 \mathrm{mV} / \mathrm{bit}$ & $2.5 \mu \mathrm{s}$ & 32768 & $24 \mathrm{~V}$ & $1.92 \mathrm{~A}$ & $25 \Omega$ \\
\hline
\end{tabular}




\section{Table 3.5}

Final system parameters for the optimal reference surface control in a dc-dc boost converter for step change in load.

\begin{tabular}{|c|c|c|c|c|c|c|c|c|}
\hline$V_{\text {in }}$ & $\mathrm{L}$ & $\mathrm{C}$ & ADC resolution & ADC delay & Chip Memory & $v_{\text {out }}$ & $i_{\text {in }}$ & $R_{\text {load }}$ \\
\hline $12 \mathrm{~V}$ & $1 \mathrm{mH}$ & $100 \mu \mathrm{F}$ & $38 \mathrm{mV} / \mathrm{bit}$ & $2.5 \mu \mathrm{s}$ & 32768 & $24 \mathrm{~V}$ & $3.84 \mathrm{~A}$ & $12.5 \Omega$ \\
\hline
\end{tabular}

\subsubsection{Stability analysis for the non-linear optimal surface}

The surface shown in Fig. 3.25 can be approximated by a quadratic function

$$
v_{\text {out }}=3.455 i_{\text {in }}^{2}-19.56 i_{\text {in }}+48.24 \text {. }
$$

The comparison of this approximate quadratic function with the original non-linear optimal surface is shown in Fig. 3.26, A general quadratic function of the form

$$
v_{\text {out }}=a i_{\text {in }}^{2}+b i_{\text {in }}+c
$$

may be used to derive a generalized stability condition for non-linear optimal surfaces generated in a dc-dc converter for step changes in loads. $a, b$ and $c$ are the coefficients of the quadratic function. This derivation will generate the condition of stability for any non-linear optimal surface for the path objective function defined in $(3.25)$ and then show that the non-linear optimal surface for the example system considered is stable. 


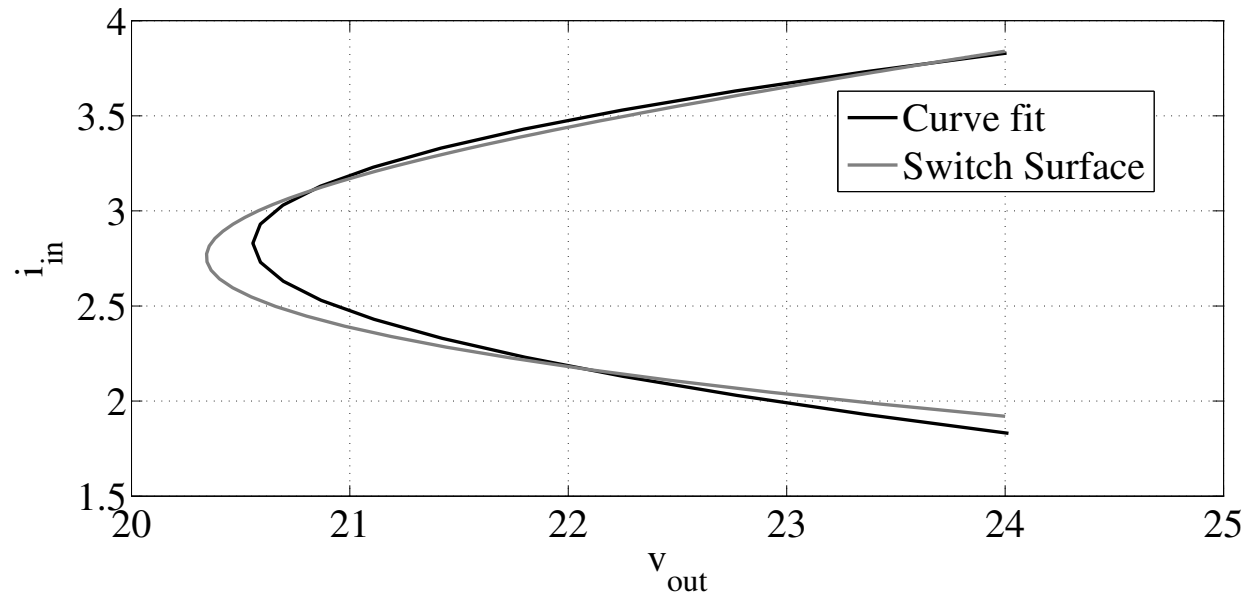

Figure 3.26: Comparison of the original non-linear optimal surface with the approximated quadratic function in a state-plane plot for the optimal reference surface control in a dc-dc boost converter.

The dynamic equations for a dc-dc boost converter is shown in (3.1). The switching control law for the dc-dc boost converter is shown in (3.7). Equation (3.9) is the required condition for stability. A non-linear optimal surface may be defined as

$$
s=v_{\text {out }}-a i_{\text {in }}^{2}-b i_{\text {in }}-c=0 .
$$

The derivative of the surface is given by

$$
\dot{s}=-2 a i_{\text {in }} \dot{i}_{\text {in }}-b \dot{i}_{\text {in }}+\dot{v}_{\text {out }} .
$$

Substituting (3.1) in (3.36),

$$
\dot{s}=\frac{2 a(1-q) i_{\text {in }} v_{\text {out }}}{L}-\frac{2 a i_{\text {in }} V_{\text {in }}}{L}-\frac{b V_{\text {in }}}{L}+\frac{b(1-q) v_{\text {out }}}{L}+\frac{(1-q) i_{\text {in }}}{C}-\frac{v_{\text {out }}}{C R} .
$$


Using (3.7) in (3.37), for the region $s<0$,

$$
\dot{s}=-\frac{2 a i_{i n} V_{i n}}{L}-\frac{b V_{i n}}{L}-\frac{v_{\text {out }}}{C R} .
$$

Therefore, for the region $s<0, \dot{s}>0$ when

$$
\frac{2 a i_{i n} V_{i n}}{L}+\frac{b V_{\text {in }}}{L}+\frac{v_{\text {out }}}{C R}<0 .
$$

For the region $s>0$,

$$
\dot{s}=\frac{2 a i_{\text {in }} v_{\text {out }}}{L}-\frac{2 a i_{\text {in }} V_{\text {in }}}{L}-\frac{b V_{\text {in }}}{L}+\frac{b v_{\text {out }}}{L}+\frac{i_{\text {in }}}{C}-\frac{v_{\text {out }}}{C R} .
$$

For the region $s>0, \dot{s}<0$ when

$$
\frac{2 a i_{\text {in }} v_{\text {out }}}{L}-\frac{2 a i_{\text {in }} V_{\text {in }}}{L}-\frac{b V_{\text {in }}}{L}+\frac{b v_{\text {out }}}{L}+\frac{i_{\text {in }}}{C}-\frac{v_{\text {out }}}{C R}<0 .
$$

Equations (3.39) and (3.41) are the required stability criteria for the region $s<0$ and $s>0$ respectively. For the example dc-dc boost converter considered for this analysis, the system parameters are shown in Table 3.4 and 3.5. Using these parameters, for $s<0,(3.38)$ reduces to

$$
\dot{s}=234720-800 v_{\text {out }}-82920 i_{\text {in }} .
$$


For the region $s>0$, (3.40) reduces to

$$
\dot{s}=234720+6910 i_{\text {in }} v_{\text {out }}-20360 v_{\text {out }}-72920 i_{\text {in }} .
$$

The phase portrait for the example dc-dc boost converter for a region around the sliding surface is shown in Fig. 3.27. The example dc-dc boost converter system has an initial equilibrium point at $(24,1.92)$ and a final equilibrium point at $(24,3.84)$, as shown in Table 3.4. The phase portrait shows that in a region around the surface and near the equilibrium points, the states are attracted to the surface obeying the switching control law shown in (3.7). However there are regions in the phase portrait where stability is not guaranteed. For the purpose of this work, the region of operation is around the surface near the equilibrium points, which exclude those regions where stability is not guaranteed. Therefore it may be concluded that the sliding surface under consideration for this dc-dc boost converter system is stable for the region of operation around the surface and the states are attracted to the surface.

\subsubsection{Simulation results for the optimal reference surface}

A simulation for the system with the reference optimal surface is done with the system parameters shown in Table 3.4 and Table 3.5. The objective is to drive the input current 


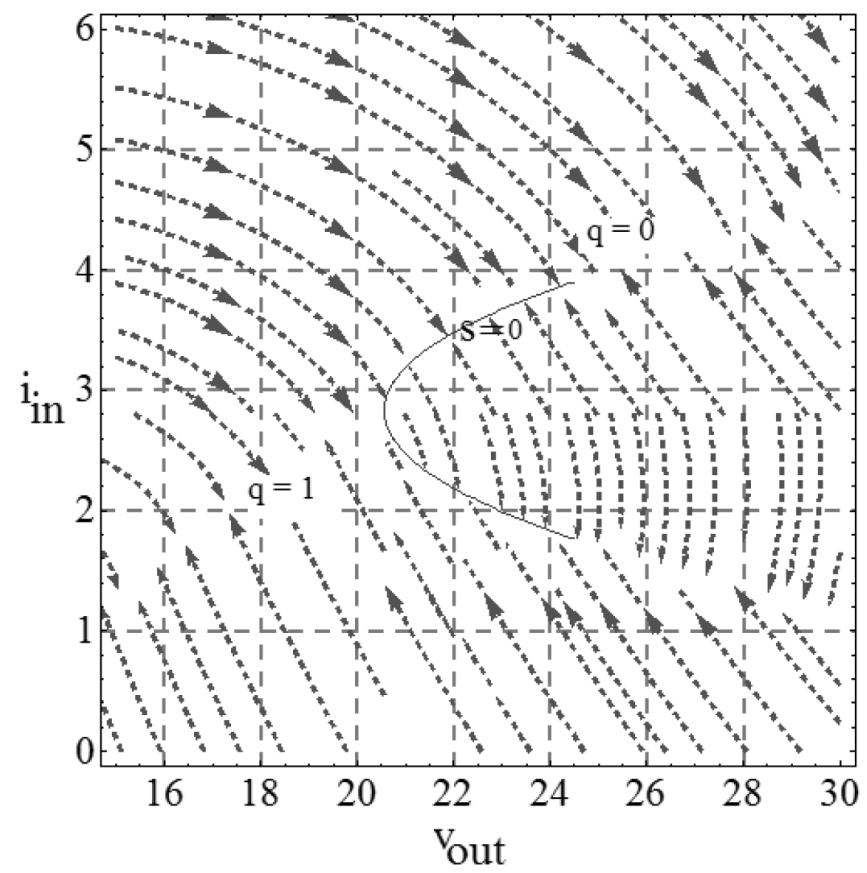

Figure 3.27: Phase portrait of dc-dc boost converter states for the non-linear optimal reference sliding surface.

and output voltage state trajectories from an initial equilibrium point to a final equilibrium point via the optimal surface shown in Fig. 3.25, Based on the values shown in Table 3.4 and Table 3.5, the initial equilibrium point in the state-plane plot is $(24 \mathrm{~V}, 1.92 \mathrm{~A})$ and the final equilibrium point is $(24 \mathrm{~V}, 3.84 \mathrm{~A})$.

The simulation results are shown in Fig. 3.28 and Fig. 3.29, Fig. 3.28 shows the variation of the input current and output voltage states with time. A step change in load is given at $t=0.005 s$. It may be noted that the input current and output voltage states reach the new equilibrium point from the old equilibrium point when the step change in load occurs, thereby validating the control strategy. Fig. 3.29 shows the state-plane plot of the output 

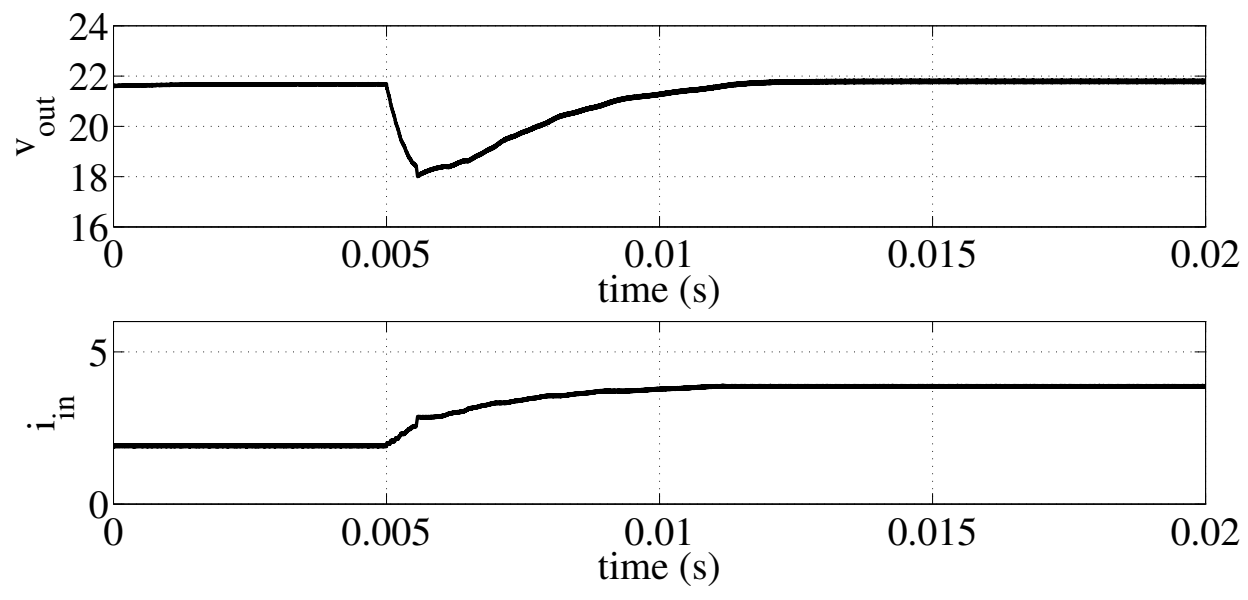

Figure 3.28: Time domain plot for the digital sliding mode control strategy for an optimal reference surface for a step change in load at $t=0.005 \mathrm{~s}$ in a dc-dc boost converter.

voltage and input current states along with the reference optimal sliding surface. The control strategy drives the state trajectories to the surface, compels the states to stay on the surface and drives them to the desired equilibrium point.

\subsubsection{Experimental results for the optimal reference surface}

The system parameters in Table 3.4 and Table 3.5 are used in an experimental set-up to validate the control strategy in a real-time hardware. The optimal surface shown in Fig. 3.25 is programmed into 32768 memory locations of the EEPROM and the ADC converter 


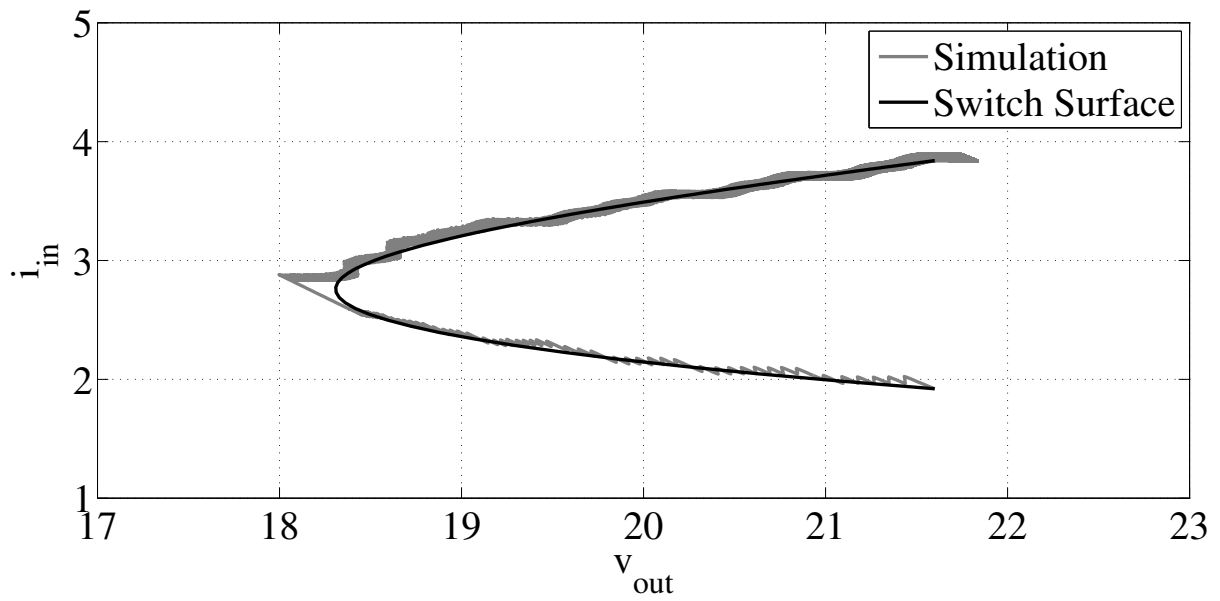

Figure 3.29: State-plane plot for the digital sliding mode control strategy for an optimal reference surface for a step change in load in a dc-dc boost converter.

is set for a propagation delay of $2.5 \mu \mathrm{s}$. In this set up, 15 input pins of the EEPROM are used to accommodate the signals coming from the two ADC converters along with the load state feedback. Fig. 3.30 shows the variation of the input current, output voltage and switch states with time. Fig. 3.31 shows the state trajectories in the steady state before any step change in load occurs. Fig. 3.32 shows the state trajectories in the steady state after the step change in load occurs, thus showing that the system states reach the desired equilibrium point. Fig. 3.33 shows the state-plane plot obtained from the raw experimental data during the transient. 


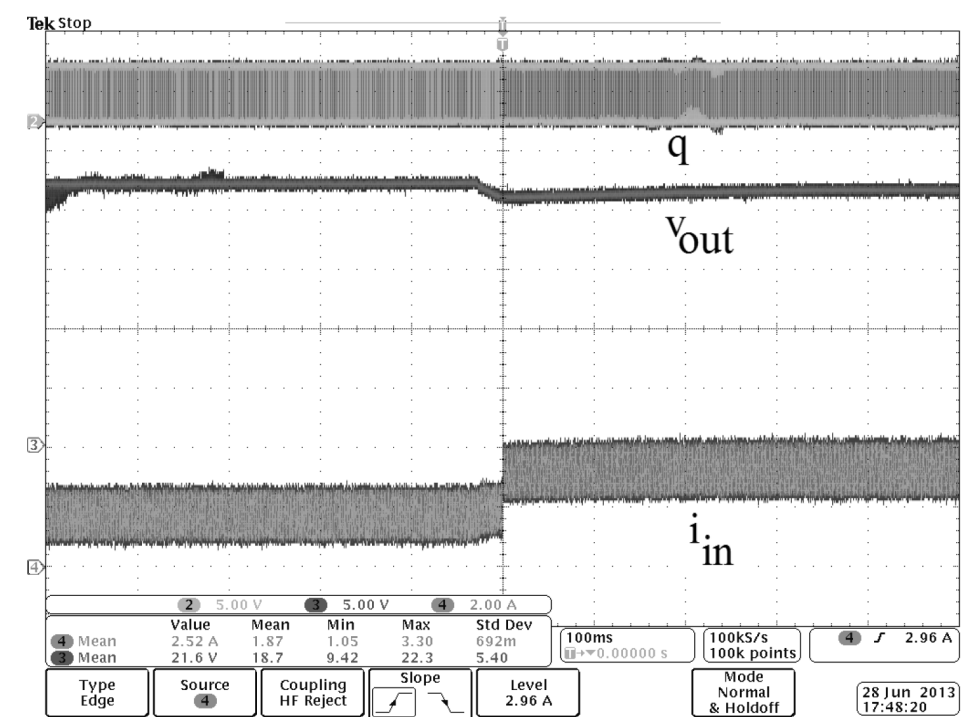

Figure 3.30: Experimental time domain plot for the digital sliding mode control strategy for an optimal reference surface for a step change in load in a dc-dc boost converter.

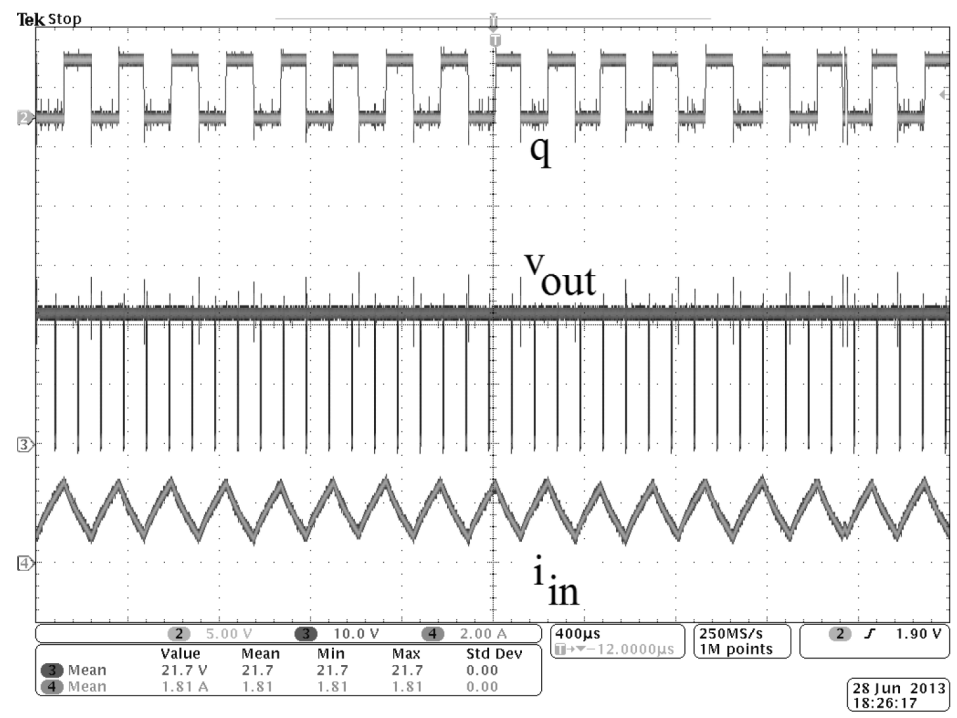

Figure 3.31: Experimental time domain plot in the steady state for the digital sliding mode control strategy for an optimal reference surface before the step change in load is applied in a dc-dc boost converter. 


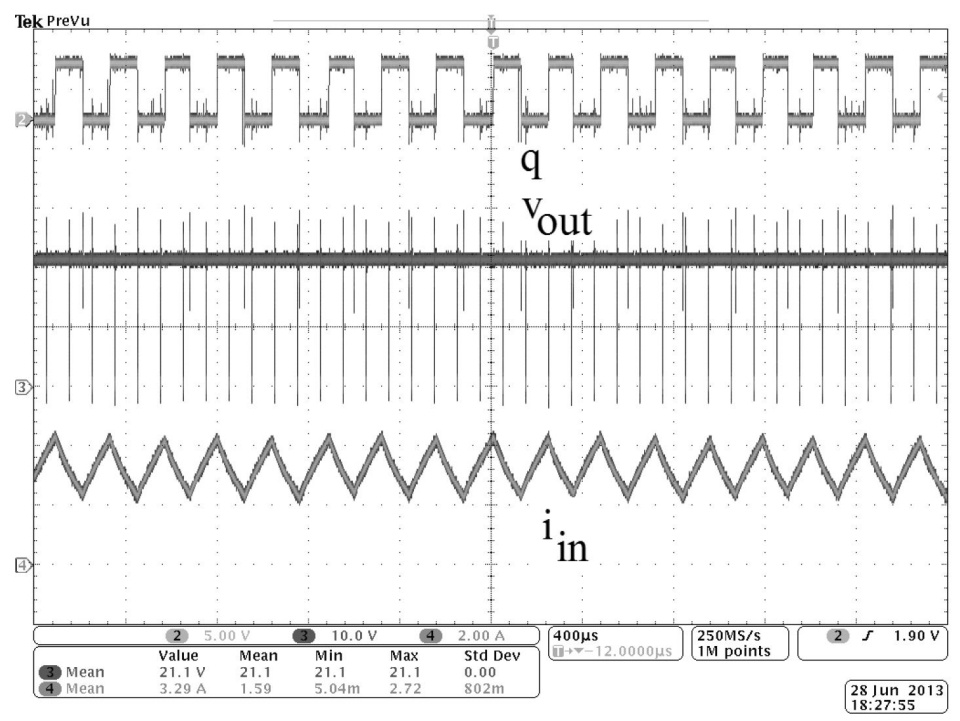

Figure 3.32: Experimental time domain plot in the steady state for the digital sliding mode control strategy for an optimal reference surface after the step change in load is applied in a dc-dc boost converter.

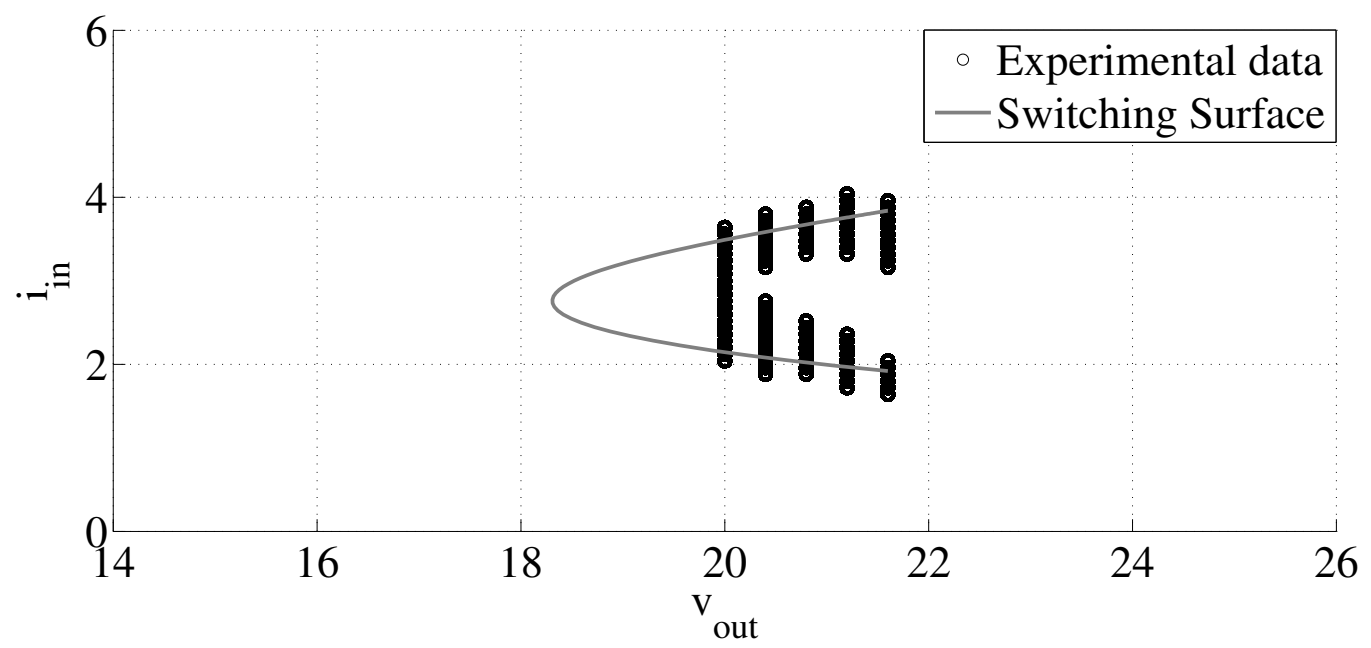

Figure 3.33: State-plane plot for the digital sliding mode control strategy for an optimal reference surface for a step change in load in a dc-dc boost converter with raw experimental data. 


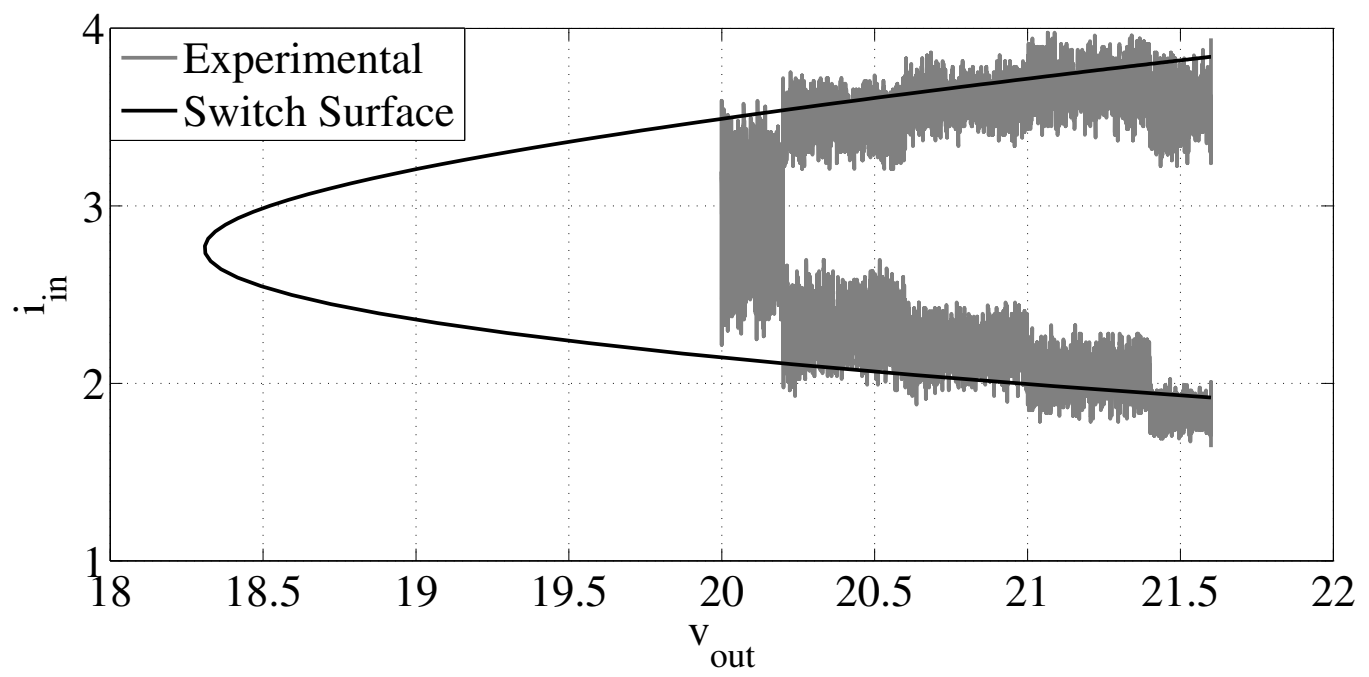

Figure 3.34: State-plane plot for the digital sliding mode control strategy for an optimal reference surface for a step change in load in a dc-dc boost converter with moving average experimental data.

For the experimental setup, the resolution for the voltage is $0.4 \mathrm{~V}$ and the resolution for current is $0.08 \mathrm{~A}$. This implies that the output voltage can only take values at a step of $0.4 \mathrm{~V}$. Fig. 3.33 shows that the output voltage takes values of $18.8 \mathrm{~V}-21.6 \mathrm{~V}$ at steps of $0.4 \mathrm{~V}$ and the input current takes values at intervals of $0.08 \mathrm{~A}$. Due to the limitation in the memory resolution, the states cannot take intermediate values. This is a limitation of this experimental set up where only 32768 memory locations could be used. This limitation can be addressed by using an EEPROM with higher number of memory locations. This will increase the memory resolution and will ensure that state trajectories take values at lesser intervals. In the simulation, the ADC was modeled with a signal delay only. Therefore, in the simulation, the signals can take continuous values. Moreover, interpolation of data takes place in the memory lookup table in the simulation. But in the experimental setup interpolation is not possible and unlike the simulation, the EEPROM reacts to sampled 
signals from the ADCs. This explains the deviation of the experimental results from the simulation results. The raw experimental data obtained during the transient is subjected to a moving average filter in MATLAB to generate the intermediate data points. The MATLAB in-built function smooth is used for this purpose. Fig. 3.34 shows the state-plane plot that uses this smoothed data. The plot shows that if the memory resolution is increased and the states take values at lesser intervals, then the states follow the surface more closely and reach the final equilibrium point.

\subsection{Discussion}

In [34], a hardware-in-loop controller is used to implement the digital controller in power converter the system. This work implemented the digital controller in an EEPROM and could successfully implement various linear and non-linear state trajectories as reference surfaces in the memory chip for different conditions, such as a cold start or a step change in load. The digital sliding mode hysteretic controller could be designed effectively such that the states slide along the reference surfaces and reach the desired equilibrium points from the initial equilibrium point, for all the different surfaces and example cases considered.

The selection of the size of the ADC and the memory size of the EEPROM is dependent on the resolution necessary for proper implementation of the sliding mode controller in the example systems chosen. Higher the resolution, better the control. There exists an 
upper limit to the resolution based on the size of the memory chip chosen for the design. The limits of ADC resolution depends on the number of ADC output bits. The higher the number of ADC bits, greater is the resolution. The choice of memory size of the chip depends on the number of state signals required and the ADC resolution.

The control strategy is made simpler in terms of hardware design by eliminating the switch state feedback to the controller to generate hysteresis. The ADC converter signal propagation delay generates the required hysteresis band. There are both upper and lower bounds to the delay in the ADC converter, beyond which this strategy will cease to work. If the delay is made very small, the hysteresis band will become extremely small and result in chattering issues on the sliding surface. On the other hand, if the delay is very big, then the memory table control logic will react to a very delayed signal and output an erroneous switch state.

The investigation on the stability of the chosen surfaces pertaining to the example systems is a very generic method of stability analysis. This method shows that the surfaces chosen are stable for the example systems considered. This analysis is a guideline for any surface stability analysis for a power electronic device based system. The stability conditions derived in this chapter are true for any dc-dc boost converter acting as a POLC for any step change in load where the objective is to regulate its terminal voltage.

This control strategy is implemented in a dc-dc boost converter. This method is equally applicable for any converter topology and also for systems having multiple power electronic 
converters. A very important criteria for this strategy to work is the selection of a stable sliding surface pertaining to the system of choice. If the surface is stable for the example system, then this strategy can be implemented in an exact similar manner.

\subsection{Conclusions}

The proposed strategy effectively controls the system under a normal operating condition as well as under transient conditions, such as a cold start or a sudden change in the load as evident from the simulation and experimental results obtained. It is also observed that since the real time implementation of this strategy does not require any on site mathematical calculations, the control action during a transient phenomena takes place in a very little time, thereby making this strategy really effective and fast. A memory chip being readily available and relatively much cheap as compared to any other complex controllers, make this strategy a low cost and effective control strategy in a microgrid. 


\section{Chapter 4}

\section{Digital sliding mode hysteretic controller in a de microgrid}

This is an extension of the work shown in Chapter 3. In this chapter, the digital sliding mode hysteretic control method is implemented in a dc microgrid where the sliding surfaces are predetermined and stored in a low cost memory chip. The sliding surfaces are derived a-priori based on a model based game-theoretic trajectories solutions of the converters. In Chapter 2, complete geometric manifolds are derived for a dc microgrid for various transient conditions. In this chapter, the control strategy is implemented in a dc- microgrid shown in Fig. 2.2 and Fig. 2.9. It was shown in Chapter 3 that use of slower analog-to-digital conversion in the process can eliminate the need to derive and implement a hysteresis band which limits the effective switching frequency. This ADC 
latency therefore, acts in favor of this controller design [84].

\subsection{Derivation of the geometric surface and implementation of the digital controller in a dc \\ microgrid}

An example de microgrid consisting of three converters as shown in Fig. 2.2 and Fig. 2.9 is considered for this work. Fig. 2.2 shows the system model in the energy-power domain and Fig. 2.9 shows the system in the voltage-current domain. The energy-power domain implies that the system states are the stored energies and the power flow into the energy storage devices. The voltage-current domain means that the system states are the output voltages of the power converters, the bus voltage and the input current to the converters. 1

For the system in Fig. 2.2, an energy balance model [1] is used to derive the game-theoretic reference surface. This derivation follows from the generalized method of derivation as discussed in Chapter 2, In the microgrid from Fig 2.2, each converter has state variables of stored energy and input power. The rate of change of the power flows in the different sections of the network are the control inputs. The state variables are therefore, the energies $w_{s 1}, w_{b u s}, w_{1}$ and $w_{2}$, and the power flows $p_{i s 1}, p_{o s 1}, p_{i n 1}$ and $p_{i n 2}$. The rate of change of

\footnotetext{
${ }^{1}$ The method of derivation of the geometric surface in Chapter 4 is adapted from [1] (O2012, IEEE. Some texts pertaining to the explanation of the method and the system are adapted from [1] (O2012, IEEE.
} 
power flowing into the source converters, the rate of change of power flowing into the common bus and the rate of change of power flowing into the POLCs are the controls for the network. During a transient condition, such as a step change in the load, the objective of the control is to maintain the terminal characteristics of the system at nominal values. For the system shown in Fig. 2.2, the objectives are to conserve the energy stored in the converters and the common bus. The set of dynamic equations for the system is given as

$$
\begin{aligned}
& \dot{w}_{s 1}=p_{i s 1}-\frac{2 w_{s 1}}{C_{s 1} R_{s 1}}-p_{o s 1} \\
& \dot{w}_{b u s}=p_{o s 1}-p_{\text {in } 1}-p_{\text {in } 2} \\
& \dot{w}_{1}=p_{\text {in } 1}-\frac{2 w_{1}}{C_{1} R_{1}} \\
& \dot{w}_{2}=p_{\text {in } 2}-\frac{2 w_{2}}{C_{2} R_{2}} \\
& \dot{p}_{\text {is } 1}=u_{11} \\
& \dot{p}_{\text {os } 1}=u_{21} \\
& \dot{p}_{\text {in } 1}=u_{31} \\
& \dot{p}_{\text {in } 2}=u_{32} .
\end{aligned}
$$

The rate of change of the power flow are the controls $u_{11}, u_{21}, u_{31}$ and $u_{32}$ as shown in (4.1).

To derive the game-theoretic optimal state trajectories of the system, an objective function of the general form

$$
J=\int_{0}^{t_{f}} g(x) d t
$$


is chosen [86]. The choice of the path objective is

$$
\begin{gathered}
g(x)=\left(w_{s 1}-w_{\text {s1 nom }}\right)^{2}+\left(w_{\text {bus }}-w_{\text {busnom }}\right)^{2}+ \\
\left(w_{1}-w_{1 \text { nom }}\right)^{2}+\left(w_{2}-w_{2 \text { nom }}\right)^{2}+u_{11}{ }^{2}+ \\
u_{21}{ }^{2}+u_{31}{ }^{2}+u_{32}{ }^{2} .
\end{gathered}
$$

$w_{\text {s1nom }}, w_{\text {busnom }}, w_{1 \text { nom }}$ and $w_{2 \text { nom }}$ are the nominal values of the respective energy functions. The path objective (4.3) seeks to keep the energy as close to nominal values as possible and the rate of change of the power flow functions are minimized. The power flow functions are made states to ensure that they are at least continuous from their nominal values, i.e. they should not change rapidly with time. It should be noted that in the following examples, equal weight is given to all the components of the objectives. Each energy storage device in the system is a player having a defined objective. The combined objectives of all the different players form a differential game. During a transient condition the objective of the game-theoretic control is to make the state trajectories travel from the initial operating conditions to the new steady-state conditions based on the minimization of the objective 
function (4.2). The initial conditions for the system are

$$
\begin{aligned}
& w_{s 1}(0)=w_{\text {s1nom }} \\
& w_{\text {bus }}(0)=w_{\text {busnom }} \\
& w_{1}(0)=w_{1 \text { nom }} \\
& w_{2}(0)=w_{2 n o m} \\
& p_{\text {is } 1}(0)=p_{\text {is } 1 o} \\
& p_{\text {os } 1}(0)=p_{\text {os } 1 o} \\
& p_{\text {in } 1}(0)=p_{\text {in } 1 o} \\
& p_{\text {in } 2}(0)=p_{\text {in } 2 o} .
\end{aligned}
$$

To simplify the objectives around the nominal operating points, (4.3) is modified to

$$
\begin{gathered}
g(x(t))=\left(\Delta w_{s 1}\right)^{2}+\left(\Delta w_{\text {bus }}\right)^{2}+\left(\Delta w_{1}\right)^{2}++\left(\Delta w_{2}\right)^{2} \\
u_{11}{ }^{2}+u_{21}{ }^{2}+u_{31}{ }^{2}+u_{32}{ }^{2}
\end{gathered}
$$

where

$$
\begin{aligned}
& \Delta w_{s 1}=\left(w_{\text {s1 }}-w_{\text {s1 nom }}\right) \\
& \Delta w_{\text {bus }}=\left(w_{\text {bus }}-w_{\text {busnom }}\right) \\
& \Delta w_{1}=\left(w_{1}-w_{1 \text { nom }}\right) \\
& \Delta w_{2}=\left(w_{2}-w_{2 \text { nom }}\right) .
\end{aligned}
$$


The state equations for the system are then

$$
\begin{aligned}
& \dot{\Delta w_{s 1}}=p_{\text {is } 1}-\frac{2\left(\Delta w_{s 1}+w_{s 1 \text { nom }}\right)}{C_{s 1} R_{s 1}}-p_{\text {os } 1} \\
& \dot{\Delta w_{\text {bus }}}=p_{\text {os } 1}-p_{\text {in } 1}-p_{\text {in } 2} \\
& \dot{\Delta w_{1}}=p_{\text {in } 1}-\frac{2\left(\Delta w_{1}+w_{1 n o m}\right)}{C_{1} R_{1}} \\
& \dot{\Delta w_{2}}=p_{\text {in } 2}-\frac{2\left(\Delta w_{2}+w_{2 n o m}\right)}{C_{2} R_{2}} \\
& \dot{p}_{\text {is } 1}=u_{11} \\
& \dot{p}_{\text {os } 1}=u_{21} \\
& \dot{p}_{\text {in } 1}=u_{31} \\
& \dot{p}_{\text {in } 2}=u_{32} .
\end{aligned}
$$

The initial conditions are also set to

$$
\begin{array}{ll}
\Delta w_{s 1}(0) & =0 \\
\Delta w_{\text {bus }}(0) & =0 \\
\Delta w_{1}(0) & =0 \\
\Delta w_{2}(0) & =0 \\
p_{\text {is } 1}(0) & =p_{\text {is } 1 o} \\
p_{\text {os } 1}(0) & =p_{\text {os } 1 o} \\
p_{\text {in } 1}(0) & =p_{\text {in } 1 o} \\
p_{\text {in } 2}(0) & =p_{\text {in } 2 o} .
\end{array}
$$

The problem now consists of the state equations (4.7), the objective function (4.2) and 
the initial conditions (4.8). For the solution of the game-theoretic control problem, Pontryagin's minimum principle [18, 86] is used. This minimum principle is capable of providing only open loop solutions [86]. However, the open loop solutions of various a-priori events will be used to form a control manifold as the basis of a real-time feedback control algorithm. The Hamiltonian for the path minimization is

$$
\begin{aligned}
& H=\left(\Delta w_{s 1}\right)^{2}+\left(\Delta w_{\text {bus }}\right)^{2}+\left(\Delta w_{1}\right)^{2}+\left(\Delta w_{2}\right)^{2}+ \\
& u_{11}^{2}+u_{21}^{2}+u_{31}^{2}+u_{32}^{2}+ \\
& \lambda_{11}\left(p_{\text {is } 1}-\frac{2\left(\Delta w_{s 1}+w_{s 1 n o m}\right)}{C_{s 1} R_{s 1}}-p_{\text {os } 1}\right)+ \\
& \lambda_{2}\left(p_{\text {osi }}-p_{\text {in } 1}-p_{\text {in } 2}\right)+ \\
& \lambda_{31}\left(p_{\text {in } 1}-\frac{2\left(\Delta w_{1}+w_{1 n o m}\right)}{C_{1} R_{1}}\right)+ \\
& \lambda_{32}\left(p_{\text {in } 2}-\frac{2\left(\Delta w_{2}+w_{2 n o m}\right)}{C_{2} R_{2}}\right)+ \\
& \lambda_{41} u_{11}+\lambda_{51} u_{21}+\lambda_{61} u_{31}+\lambda_{62} u_{32}
\end{aligned}
$$

where $\lambda_{11}, \lambda_{2}, \lambda_{31}, \lambda_{32}, \lambda_{41}, \lambda_{51}, \lambda_{61}$ and $\lambda_{62}$ are the co-states [86]. The solution for the controls $u_{11}{ }^{*}, u_{21}{ }^{*}, u_{31}{ }^{*}$ and $u_{32}{ }^{*}$ are found from

$$
\begin{aligned}
& \frac{d H}{d u_{11}}=0 \\
& \frac{d H}{d u_{21}}=0 \\
& \frac{d H}{d u_{31}}=0 \\
& \frac{d H}{d u_{32}}=0
\end{aligned}
$$


and the co-states are found from

$$
\begin{aligned}
& \dot{\lambda}_{11}=-\frac{d H}{d\left(\Delta w_{s 1}\right)} \\
& \dot{\lambda}_{2}=-\frac{d H}{d\left(\Delta w_{\text {bus }}\right)} \\
& \dot{\lambda}_{31}=-\frac{d H}{d\left(\Delta w_{1}\right)} \\
& \dot{\lambda}_{32}=-\frac{d H}{d\left(\Delta w_{2}\right)} \\
& \dot{\lambda}_{41}=-\frac{d H}{d\left(p_{i s 1}\right)} \\
& \dot{\lambda}_{51}=-\frac{d H}{d\left(p_{\text {os } 1}\right)} \\
& \dot{\lambda}_{61}=-\frac{d H}{d\left(p_{\text {in } 1}\right)} \\
& \dot{\lambda}_{62}=-\frac{d H}{d\left(p_{\text {in } 2}\right)} .
\end{aligned}
$$


Thus the augmented set of equations are

$$
\begin{aligned}
& \dot{\Delta w_{s 1}}=p_{i s 1}-\frac{2\left(\Delta w_{s 1}+w_{s 1 n o m}\right)}{C_{s 1} R_{s 1}}-p_{o s 1} \\
& \dot{\Delta}_{\text {bus }}=p_{o s 1}-p_{\text {in } 1}-p_{\text {in } 2} \\
& \dot{\Delta w_{1}}=p_{\text {in } 1}-\frac{2\left(\Delta w_{1}+w_{1 n o m}\right)}{C_{1} R_{1}} \\
& \dot{\Delta}_{2}=p_{i n 2}-\frac{2\left(\Delta w_{2}+w_{2 n o m}\right)}{C_{2} R_{2}} \\
& \dot{p}_{i s 1} \quad=-\frac{\lambda_{41}}{2} \\
& \dot{p}_{\text {os } 1}=-\frac{\lambda_{51}}{2} \\
& \dot{p}_{\text {in } 1}=-\frac{\lambda_{61}}{2} \\
& \dot{p}_{\text {in } 2}=-\frac{\lambda_{62}}{2} \\
& \dot{\lambda}_{11}=\frac{2 \lambda_{11}}{C_{s 1} R_{s 1}}-2 \Delta w_{s 1} \\
& \dot{\lambda}_{2}=-2 \Delta w_{\text {bus }} \\
& \dot{\lambda}_{31}=\frac{2 \lambda_{31}}{C_{1} R_{1}}-2 \Delta w_{1} \\
& \dot{\lambda}_{32}=\frac{2 \lambda_{32}}{C_{2} R_{2}}-2 \Delta w_{2} \\
& \dot{\lambda}_{41}=-\lambda_{11} \\
& \dot{\lambda}_{51}=-\lambda_{2}+\lambda_{11} \\
& \dot{\lambda}_{61}=\lambda_{2}-\lambda_{31} \\
& \dot{\lambda}_{62}=\lambda_{2}-\lambda_{32}
\end{aligned}
$$


and the boundary conditions are

$$
\begin{array}{rlrl}
\Delta w_{s 1}(0) & =0 & \lambda_{11}\left(t_{f}\right)=0 \\
\Delta w_{\text {bus }}(0) & =0 & \lambda_{2}\left(t_{f}\right)=0 \\
\Delta w_{1}(0)=0 & \lambda_{31}\left(t_{f}\right)=0 \\
\Delta w_{2}(0)=0 & \lambda_{32}\left(t_{f}\right)=0 \\
p_{\text {is } 1}(0)=p_{\text {is } 1 o} & \lambda_{41}\left(t_{f}\right)=0 \\
p_{\text {os } 1}(0)=p_{\text {os } 1 o} & \lambda_{51}\left(t_{f}\right)=0 \\
p_{\text {in } 1}(0)=p_{\text {in } 1 o} & \lambda_{61}\left(t_{f}\right)=0 \\
p_{\text {in } 2}(0)=p_{\text {in } 2 o} & \lambda_{62}\left(t_{f}\right)=0 .
\end{array}
$$

This is a two-point boundary value problem (BVP). For an optimal solution, the Hamiltonian is a minimized scalar value. The solution of (4.12) and (4.13) yields the game-theoretic control trajectories for the stored energies $w_{s 1}, w_{b u s}, w_{1}$ and $w_{2}$, the power flows $p_{i s 1}, p_{o s}, p_{i n 1}$ and $p_{i n 2}$ under a transient disturbance, such as a step change in the load, $R_{1}, R_{2}$ or both. To be implemented in real-time hardware, this trajectory needs to be 
mapped to voltage and current states. With reference to Fig 2.9, the conversion equation is

$$
\begin{aligned}
& v_{s 1}=\sqrt{\frac{2 w_{s 1}}{C_{s 1}}} \\
& v_{\text {bus }}=\sqrt{\frac{2 w_{\text {bus }}}{C_{b}}} \\
& v_{1}=\sqrt{\frac{2 w_{1}}{C_{1}}} \\
& v_{2}=\sqrt{\frac{2 w_{2}}{C_{2}}} \\
& i_{s 1}=\frac{p_{i s 1}}{V_{d c 1}} \\
& i_{o s 1}=\frac{p_{\text {os } 1}}{v_{\text {bus }}} \\
& i_{L 1}=\frac{p_{\text {in } 1}}{v_{\text {bus }}} \\
& i_{L 2}=\frac{p_{\text {in } 2}}{v_{\text {bus }}} .
\end{aligned}
$$

For the dc microgrid shown in Fig. 2.9, the system parameters are shown in Table 4.1 and Table 4.2. A transient condition in the form of step changes in the loads is considered, where both the loads $R_{1}$ and $R_{2}$ are given a step from $50 \Omega$ to $33 \Omega$. The game-theoretic control problem is solved and the reference geometric trajectories for this specific case of load change are shown in Fig. 4.1 through 4.3, Since same step change in load are applied to both the POLCs, the surfaces generated are similar.

Table 4.1

Example dc microgrid initial parameters for step change in load used for simulation.

\begin{tabular}{|c|c|c|c|c|c|c|c|c|c|c|c|}
\hline$V_{d c 1}$ & $\mathrm{~L}$ & $\mathrm{C}$ & $V_{s 1}$ & $V_{b}$ & $V_{1}$ & $V_{2}$ & $i_{s}$ & $i_{L 1}$ & $i_{L 2}$ & $R_{1}$ & $R_{2}$ \\
\hline $9 \mathrm{~V}$ & $1 \mathrm{mH}$ & $1000 \mu F$ & $12 \mathrm{~V}$ & $12 \mathrm{~V}$ & $24 \mathrm{~V}$ & $24 \mathrm{~V}$ & $2.72 \mathrm{~A}$ & $0.96 \mathrm{~A}$ & $0.96 \mathrm{~A}$ & $50 \Omega$ & $50 \Omega$ \\
\hline
\end{tabular}


Table 4.2

Example dc microgrid final parameters for step change in loads used for simulation.

\begin{tabular}{|c|c|c|c|c|c|c|c|c|c|c|c|}
\hline$V_{d c 1}$ & $\mathrm{~L}$ & $\mathrm{C}$ & $V_{s 1}$ & $V_{b}$ & $V_{1}$ & $V_{2}$ & $i_{s 1}$ & $i_{L 1}$ & $i_{L 2}$ & $R_{1}$ & $R_{2}$ \\
\hline $9 \mathrm{~V}$ & $1 \mathrm{mH}$ & $1000 \mu F$ & $12 \mathrm{~V}$ & $12 \mathrm{~V}$ & $24 \mathrm{~V}$ & $24 \mathrm{~V}$ & $4 \mathrm{~A}$ & $1.45 \mathrm{~A}$ & $1.45 \mathrm{~A}$ & $33 \Omega$ & $33 \Omega$ \\
\hline
\end{tabular}

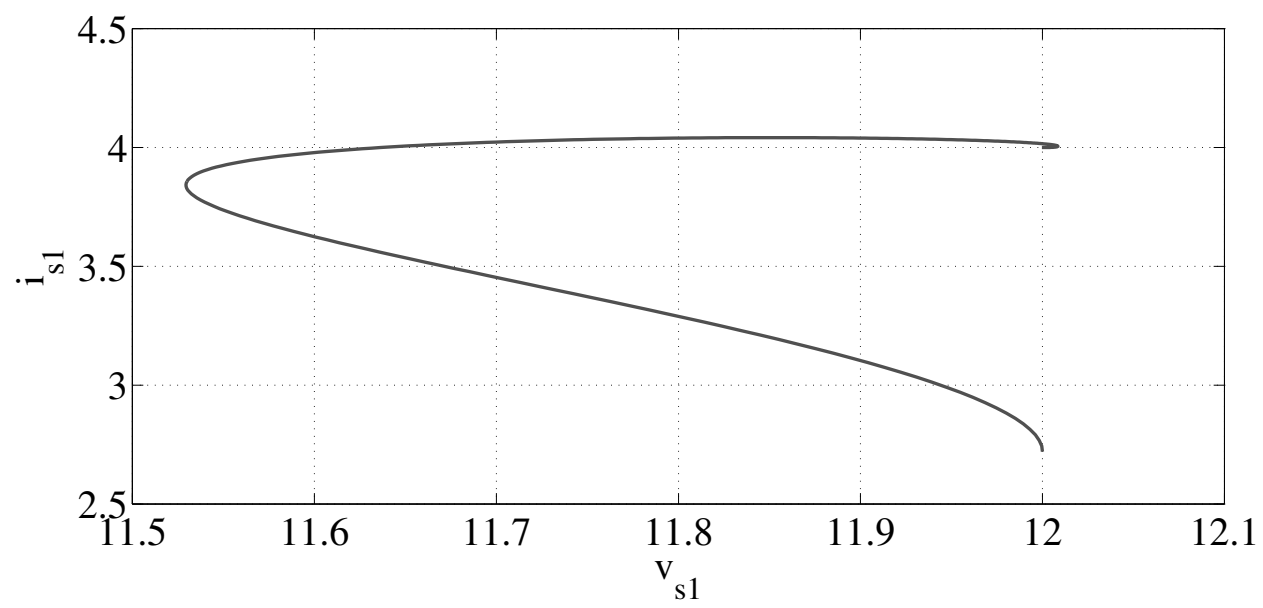

Figure 4.1: Reference game-theoretic geometric surface for the source converter in the dc microgrid.

In the dc microgrid under consideration, the objective is to make the input current and the output voltage states of the dc-dc boost converters follow the respective reference surfaces and reach the new equilibrium points corresponding to the changed load condition. As shown in Chapter 3, two 8 bit ADC converters are used and the state signals are scaled before sampling. The LSB of the ADC converter output is not used for noise issues. The output voltage resolution for all the converters is $0.4 \mathrm{~V}$. The input current resolution for the source converter is $80 \mathrm{~mA}$ and that for the POLCs is $20 \mathrm{~mA}$. For this set up, $2^{15}=32768$ 


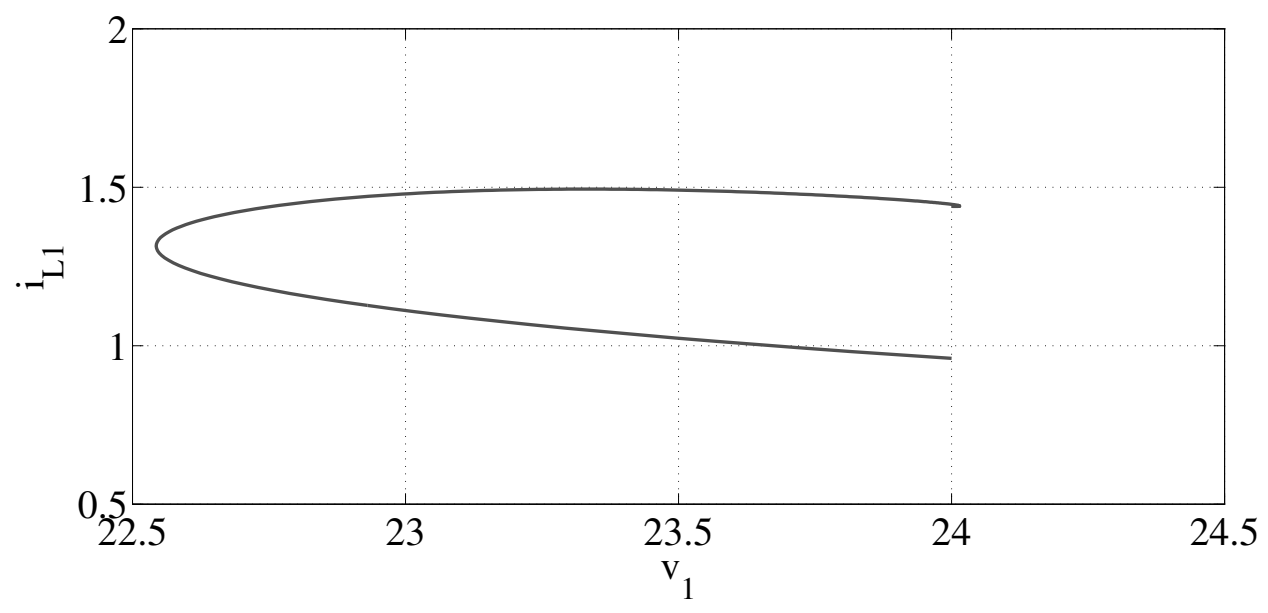

Figure 4.2: Reference game-theoretic geometric surface for the POLC 1 in the de microgrid.

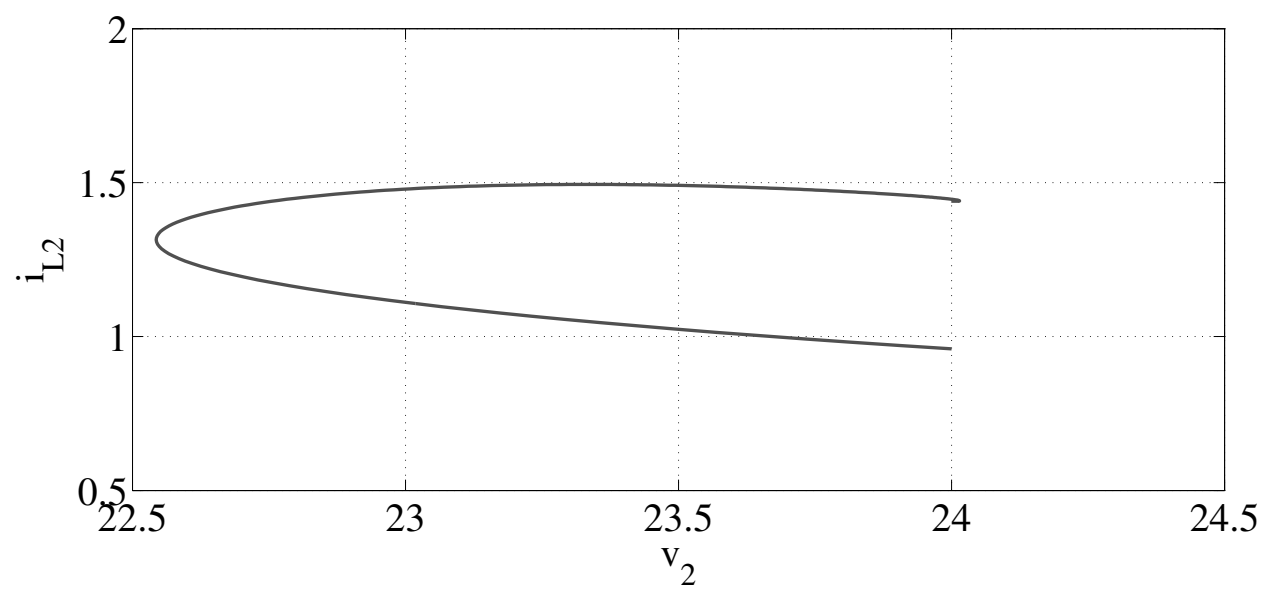

Figure 4.3: Reference game-theoretic geometric surface for the POLC 2 in the de microgrid.

memory locations of the EEPROM are used where the switch states are programmed. An additional input for the EEPROM becomes necessary to notify the EEPROM of the load condition of the system. Therefore, the EEPROM senses a step change in the load and generates the switch states accordingly. The chip program is done taking the load change into consideration. The EEPROM reads in the ADC converter output and generates the 
switch states as stored in its memory to drive the system state to the desired equilibrium points on the reference sliding surfaces. The overall control of the system requires control for the individual converters based on local information available to the controller. The control for a power converter lies in controlling the switch mode of the converter [98]. The switching for the converters are controlled by the hysteretic sliding mode control technique as described in Chapter 3 ,

The dc microgrid considered for this work has three current states and three voltage states corresponding to each power converter and an additional bus voltage state which is coupled to each converter. The reference geometric surfaces act as the sliding surfaces to which the system states should slide. Since the sliding surfaces are already available based on the specific scenario of load change, the usage of the digital control strategy will prove effective, as shown in Chapter 3. Thus, a predetermined calculation of the sliding surfaces are used in the controller design. This is beneficial in the sense that on site calculations are not necessary thus making the system model less complicated and much faster. So the surfaces shown in Fig. 4.1 through 4.3 are used as a-priori calculated surfaces and fed into a memory chip. This memory chip which contains the complete surface and the switching law in it constitutes the digital part of the total control system. The system states are fed into the memory chip and the surfaces are converted into quantized surfaces and based on these quantized system state trajectories the control law determines the switch states of each power converter. This EEPROM thus forms the control center for the hysteretic sliding mode controller. Here the input to the converter, which is a dc-dc converter are the 
input voltage and the output current. The output voltage and the input current are fed into the EEPROM via ADCs. The EEPROM which has the switching law incorporated in it generates the switch state which is fed into the converter thereby controlling the system states. The local information is also fed into the memory chip. This local information tells the EEPROM to react according to any change in the system. This information contains the condition of the load for the POLCs and each EEPROM associated to the POLCs gets to know when a step change in the load takes place. The memory chip contains all the different state trajectories for all the different scenarios of load changes. Thus, this kind of a controller is capable of generating a correct switch state necessary based on the system conditions.

Thus, it may be observed that if a predetermined sliding surface for the different scenarios of transient conditions, such as step changes in the load, along with the hysteretic sliding mode controller is fed into a memory location prior to a system operation, this kind of a digital hysteretic sliding mode control strategy is capable to drive the system states to the desired equilibrium points based on the control. 


\subsection{Stability analysis for the geometric surfaces}

The surface shown in Fig. 4.1 for the source converter can be approximated by piecewise quadratic functions

$$
\begin{aligned}
& i_{s 1}=-1.64 v_{s 1}^{2}+38.67 v_{s 1}-224.74 \\
& i_{s 1}=-0.8 v_{s 1}^{2}+16.83 v_{s 1}-83.86 .
\end{aligned}
$$

Since the surfaces for POLC 1 and POLC 2 are the same, the analysis of one of them will be sufficient to show that the other one is stable. The surface shown in Fig. 4.2 for the POLC 1 can be approximated by piecewise quadratic functions

$$
\begin{aligned}
& i_{L 1}=-0.17 v_{o 1}^{2}+8.04 v_{o 1}-92.64 \\
& i_{L 1}=0.152 v_{o 1}^{2}-7.27 v_{o 1}+87.97 .
\end{aligned}
$$

The comparison of these approximate quadratic functions with the original surfaces are shown in Fig. 4.4 and 4.5 , 


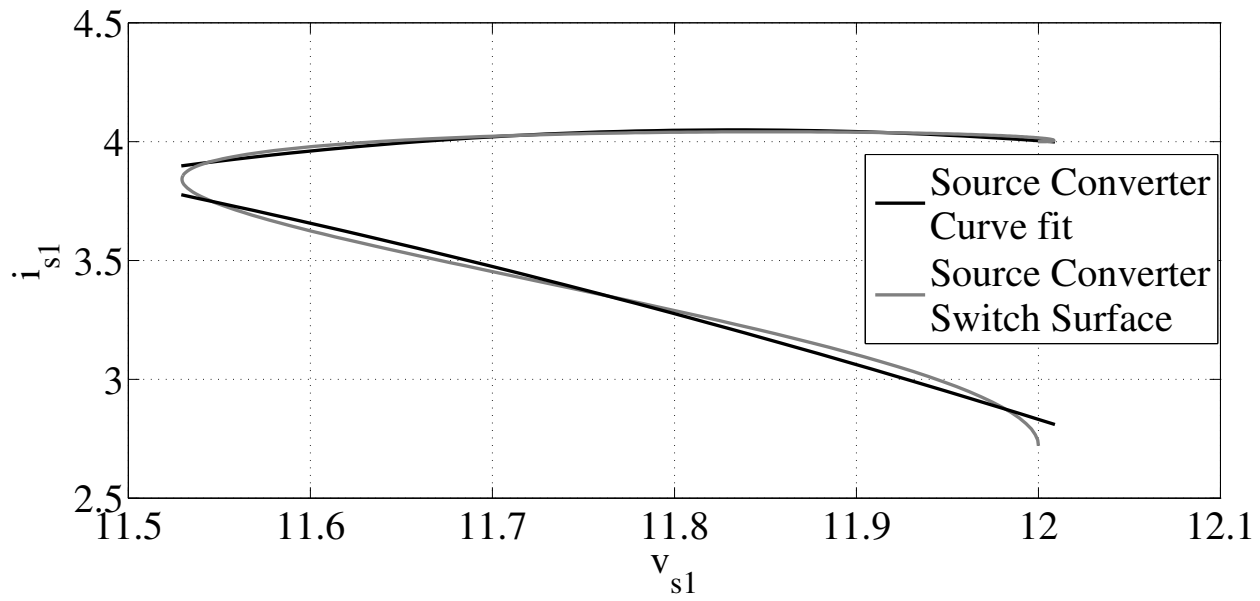

Figure 4.4: Comparison of the original surface for the source converter with the approximated piecewise quadratic functions in a state-plane plot.

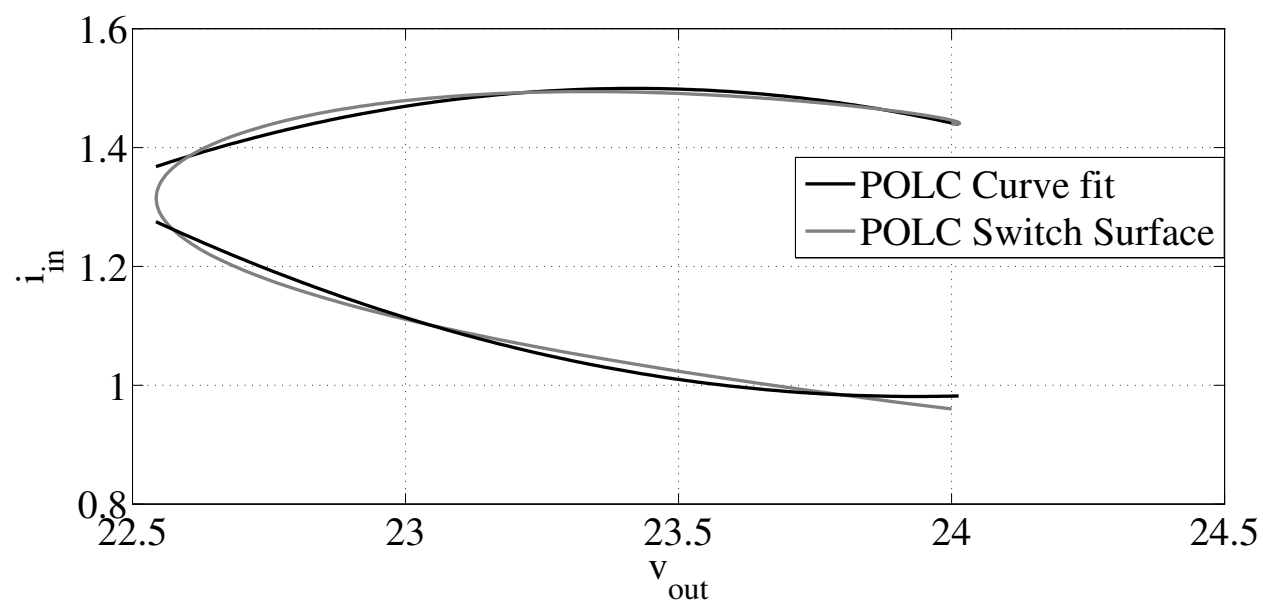

Figure 4.5: Comparison of the original surface for the POLCs with the approximated piecewise quadratic function in a state-plane plot.

A general quadratic function of the form

$$
i_{\text {in }}=a v_{\text {out }}^{2}+b v_{\text {out }}+c
$$

may be used to derive a generalized stability condition for approximated quadratic surfaces generated in a dc-dc converter for step changes in loads. $a, b$ and $c$ are the coefficients of 
the quadratic function. For the source converter, $i_{i n}=i_{s 1}$ and $v_{\text {out }}=v_{s 1}$. For the POLC $1, i_{\text {in }}=i_{L 1}$ and $v_{\text {out }}=v_{o 1}$. This derivation will generate the condition of stability for any geometric surface approximated by quadratic functions having a general form shown in (4.17) and then show that the surfaces for the example dc microgrid are stable.

The dynamic equations for a dc-dc boost converter is shown in (3.1). The switching control law for the dc-dc boost converter is shown in (3.7). Equation (3.9) is the required condition for stability. The generalized approximated surface in the state-plane diagram may be defined as

$$
s=i_{\text {in }}-a v_{\text {out }}^{2}-b v_{\text {out }}-c=0 .
$$

The derivative of the surface is given by

$$
\dot{s}=-2 a v_{\text {out }} \dot{v}_{\text {out }}-b \dot{v}_{\text {out }}+\dot{i}_{\text {in }}
$$

Substituting (3.1) in 4.19),

$$
\dot{s}=-\frac{2 a(1-q) i_{\text {in }} v_{\text {out }}}{C}-\frac{2 a v_{\text {out }}^{2}}{C R}-\frac{b(1-q) i_{\text {in }}}{C}+\frac{b v_{\text {out }}}{C R}+\frac{V_{\text {in }}}{L}-\frac{(1-q) v_{\text {out }}}{L} .
$$

Using (3.7) in 4.20), for the region $s<0$,

$$
\dot{s}=-\frac{2 a v_{\text {out }}^{2}}{C R}+\frac{b v_{\text {out }}}{C R}+\frac{V_{\text {in }}}{L} .
$$


Therefore, for the region $s<0, \dot{s}>0$ when

$$
\frac{b v_{\text {out }}}{C R}+\frac{V_{\text {in }}}{L}-\frac{2 a v_{\text {out }}^{2}}{C R}>0
$$

For the region $s>0$,

$$
\dot{s}=-\frac{2 a i_{\text {in }} v_{\text {out }}}{C}-\frac{2 a v_{\text {out }}^{2}}{C R}-\frac{b i_{\text {in }}}{C}+\frac{b v_{\text {out }}}{C R}+\frac{V_{\text {in }}}{L}-\frac{v_{\text {out }}}{L} .
$$

For the region $s>0, \dot{s}<0$ when

$$
\frac{2 a i_{\text {in }} v_{\text {out }}}{C}+\frac{2 a v_{\text {out }}^{2}}{C R}+\frac{b i_{\text {in }}}{C}-\frac{b v_{\text {out }}}{C R}-\frac{V_{\text {in }}}{L}+\frac{v_{\text {out }}}{L}>0 .
$$

Equations (4.22) and (4.24) are the required stability criteria. For the example system considered for this analysis, the system parameters are shown in Table 4.1 and 4.2, The phase portrait for the source converter for a region around the sliding surface is shown in Fig. 4.6 and the phase portrait for the POLC 1 is shown in Fig. 4.7. The phase portraits show that for a small region around the surface, the states are attracted to the surface obeying the switching control law shown in (3.7). There are regions in the phase portrait of both the source converter and POLC 1 where stability is not guaranteed. The operating points of the system are shown in Table 4.1 and 4.2 , The system operates in a region around the surface and around the equilibrium points. Hence, the regions where stability is not guaranteed is not of concern here. The phase portraits show that for the 


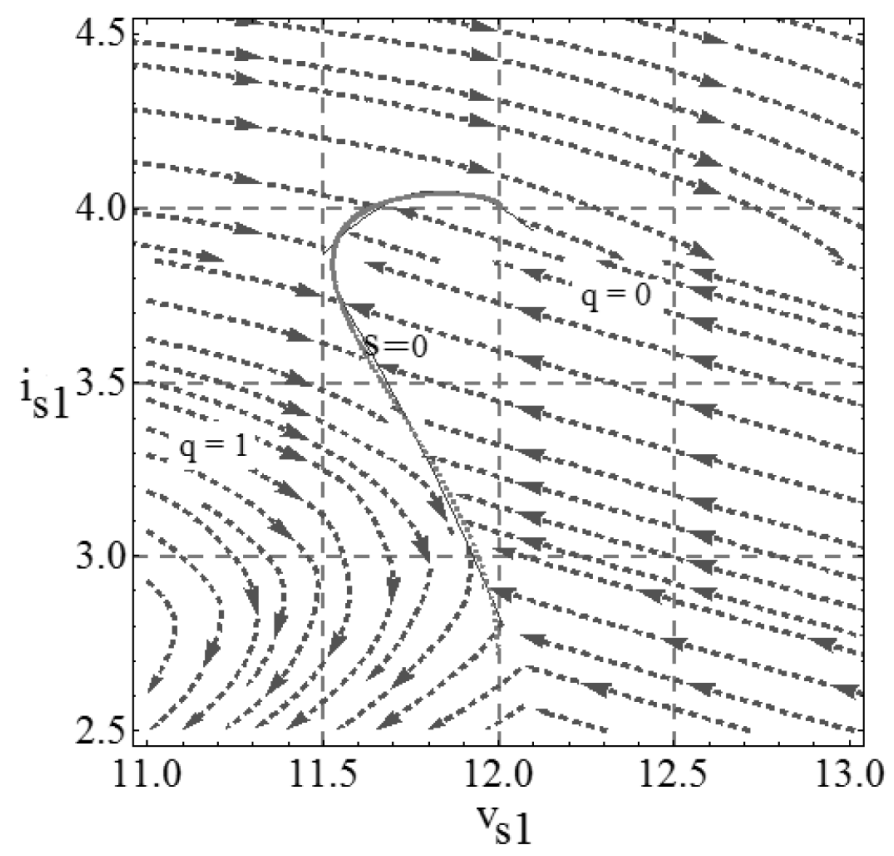

Figure 4.6: Phase portrait of source converter states in the dc microgrid for the geometric reference sliding surface.

region of operation, the states are attracted to the surface. Therefore it may be concluded that the sliding surfaces under consideration for the source converter and the POLCs in the microgrid are stable for the region of operation and the states are attracted to the surface. 


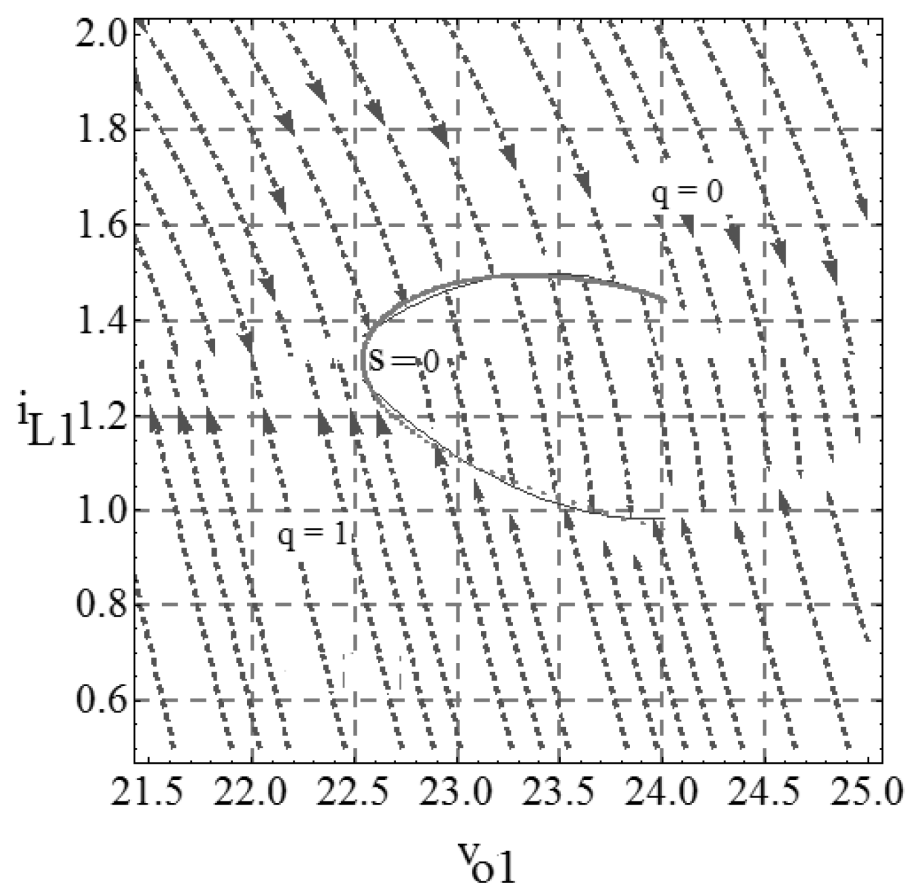

Figure 4.7: Phase portrait of POLC 1 states in the dc microgrid for the geometric reference sliding surface.

\subsection{Simulation results showing the implementation of the}

\section{digital control strategy for the de microgrid}

The circuit for the dc microgrid with three dc-dc boost converters and their control circuits is shown in Fig. 4.8, The initial and final system parameters selected are shown in Table 4.1 and Table 4.2 respectively. The system undergoes a transient in the form of a step change of both the loads $R_{1}$ and $R_{2}$ at $t=0.1 s$. The geometric sliding surfaces are pre-calculated and fed into the memory tables prior to the system model simulation. The system trajectories are shown to reach the sliding surfaces when the transient condition in the form of a step 


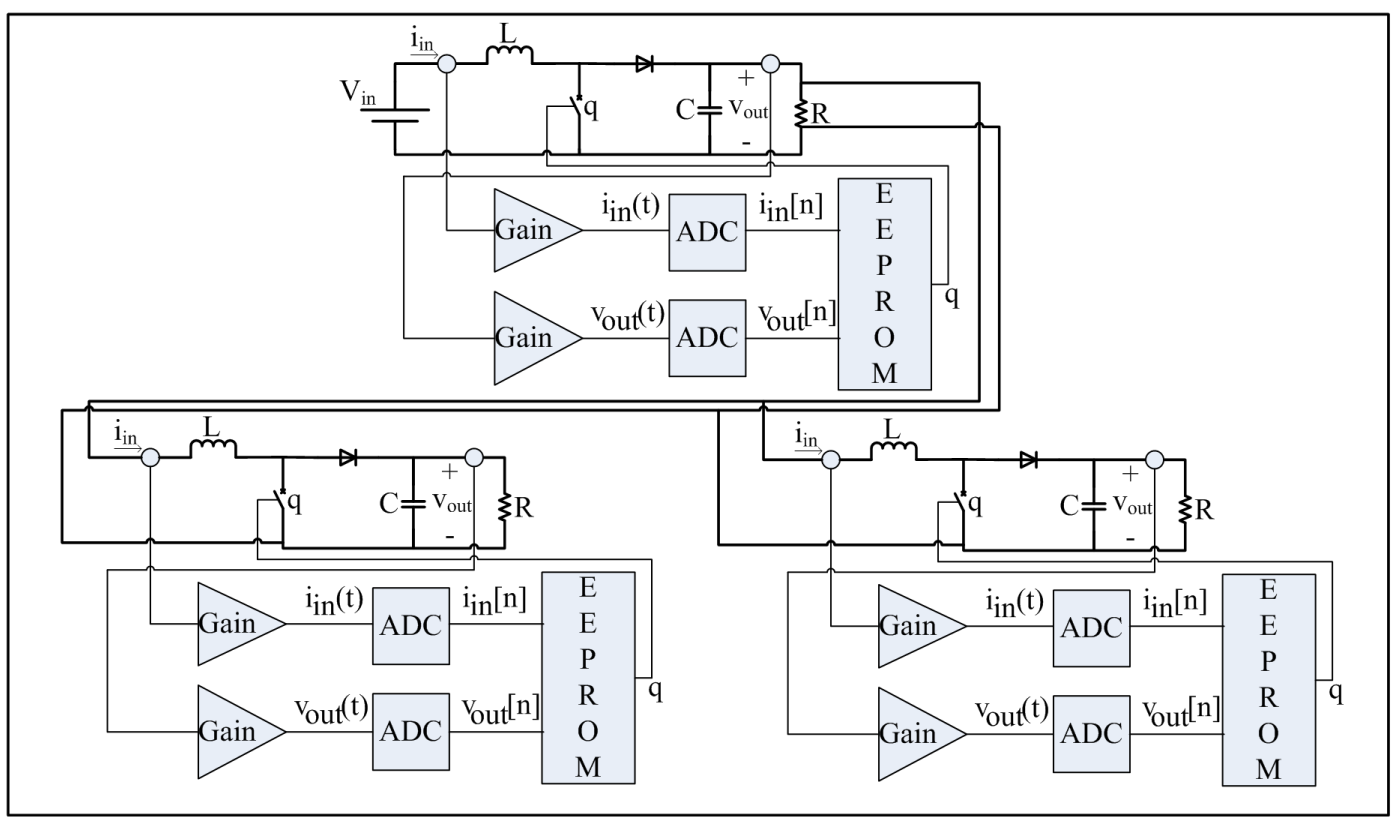

Figure 4.8: Circuit diagram for the dc microgrid.

change in the loads is simulated. The state trajectories are also shown to reach the new equilibrium point based on the predetermined sliding surfaces as fed into the memory table. The state trajectory responses are shown in Fig. 4.9 through Fig. 4.12, 

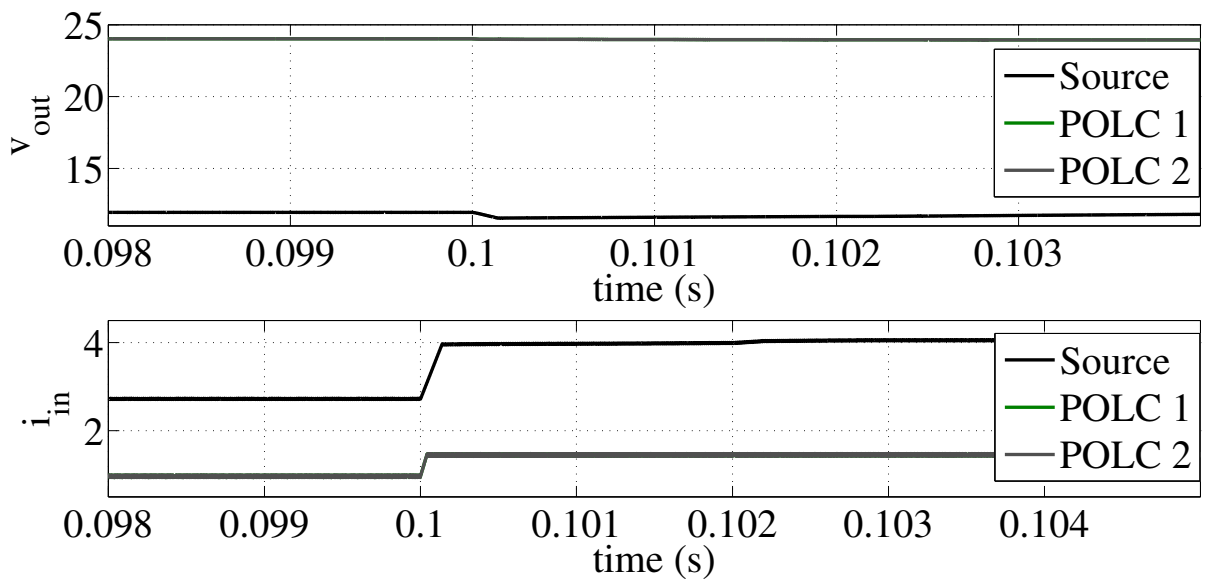

Figure 4.9: Current and voltage profiles for the dc-dc boost converters in the dc microgrid for a step change in load at $t=0.1 \mathrm{~s}$.

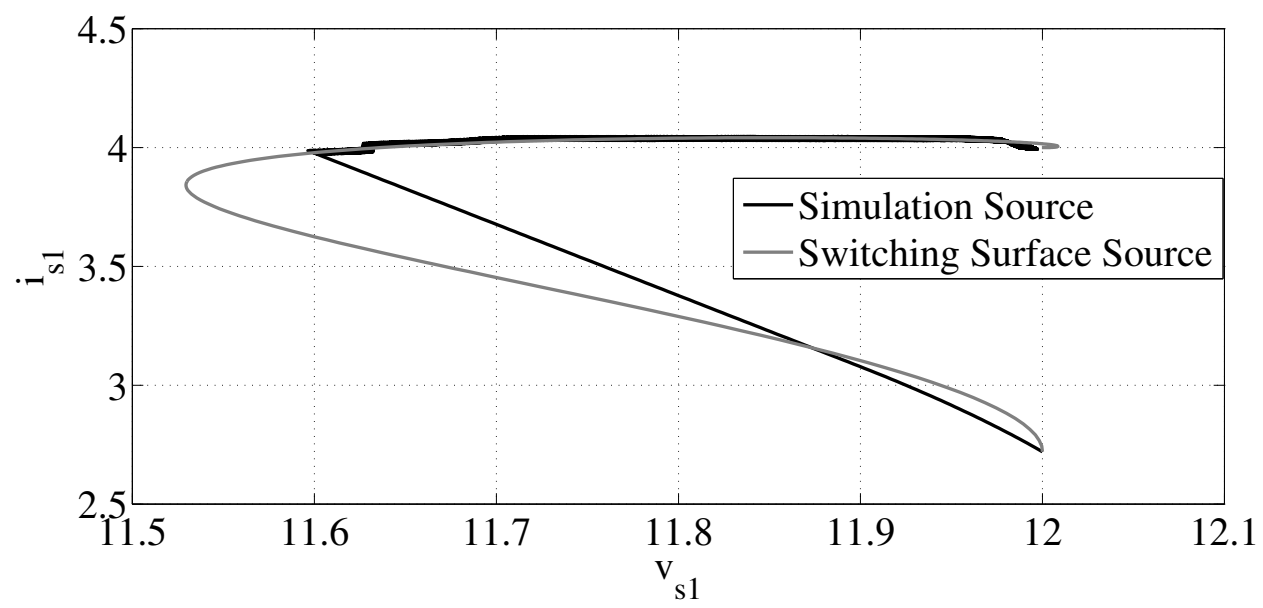

Figure 4.10: State trajectories for the source converter in the dc microgrid for a step change in load.

Fig. 4.9 shows the variation of the voltage and the current states for the converters with time. It may be observed that the state trajectories respond to the step change in the load and adapted according to the control law set in the digital controller. Fig. 4.10 to Fig. 4.12 shows the reference surfaces based on the a-priori calculations for this particular example 


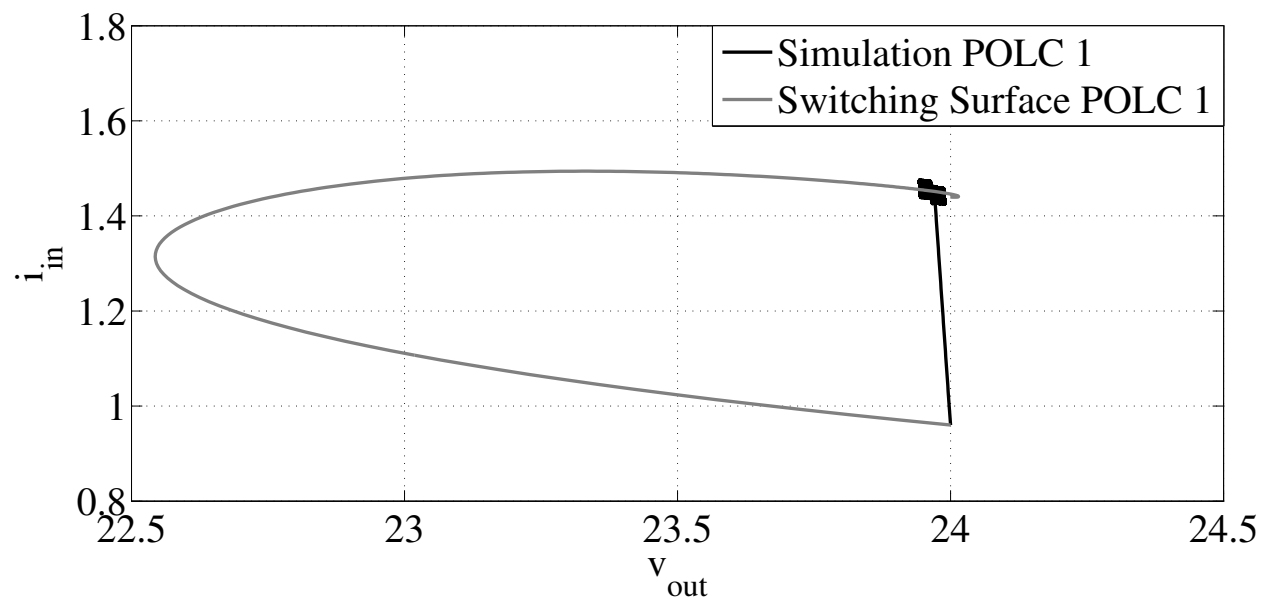

Figure 4.11: State trajectories for POLC 1 in the dc microgrid for a step change in load.

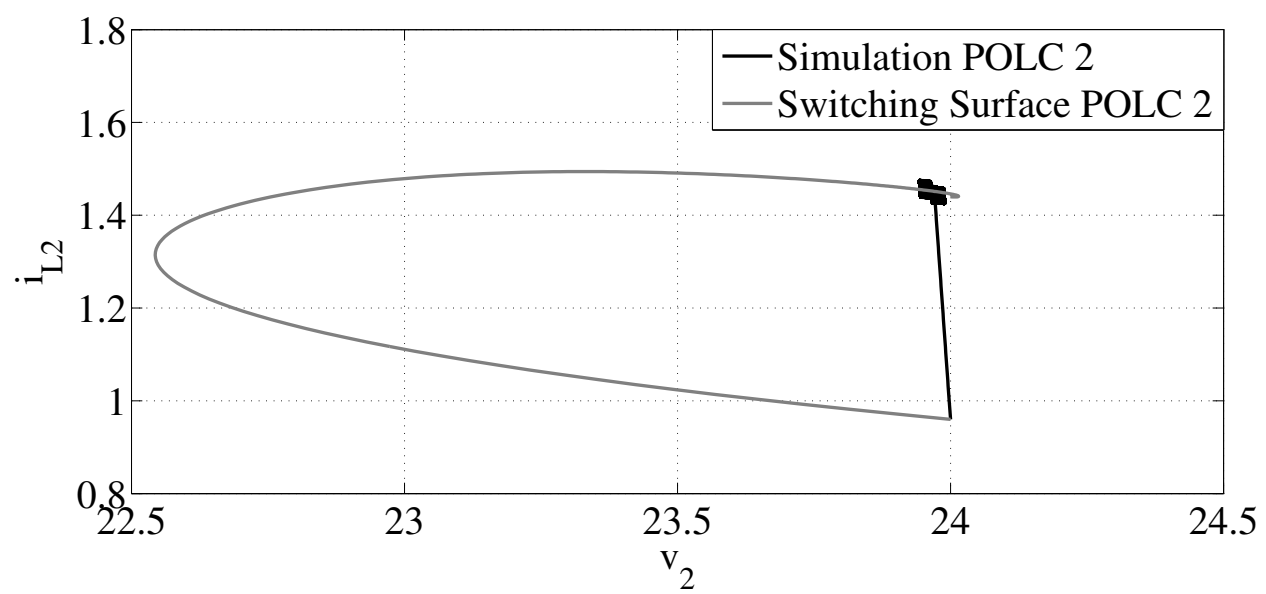

Figure 4.12: State trajectories for POLC 2 in the dc microgrid for a step change in load.

case along with the state trajectories based on the proposed controller. Thus it may be observed that the proposed controller actually works and is capable of driving the system states to the required reference surface based on the a-priori calculations. The quantization effects of the digital controller is also evident in the simulation as observed in the Fig. 4.10 to Fig. 4.12 , 


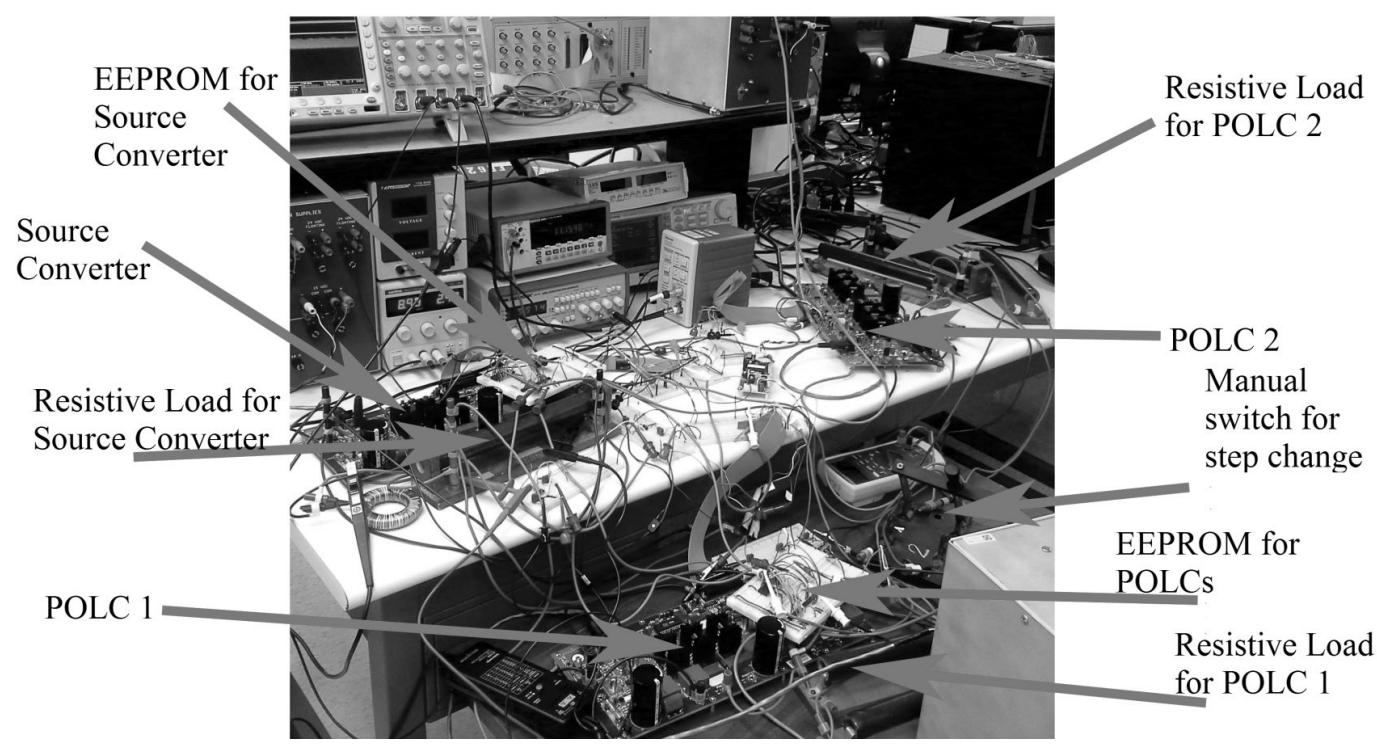

Figure 4.13: Experimental set up for the digital sliding mode hysteretic controller implementation in a dc microgrid for a step change in load.

\subsection{Experimental results for the dc microgrid with reference geometric surfaces for step change in the}

\section{loads}

The system parameters in Table 4.1 and Table 4.2 are used in an experimental set-up to validate the control strategy in a real-time hardware. The complete experimental set up is shown in Fig. 4.13 
The geometric reference surfaces shown in Fig. 4.1 through 4.3 are programmed into 32768 memory locations of three EEPROMs and the ADC converters are set for propagation delays of $2.5 \mu \mathrm{s}$ each. In this set up, 15 input pins of each EEPROM are used to accommodate the signals coming from the ADC converters along with the load state. Fig. 4.14 shows the variation of the input current, output voltage and switch states of the source converter with time. Fig. 4.15 shows the state trajectories of the source converter in the steady state before any step change in load occurs. Fig. 4.16 shows the source converter state trajectories in the steady state after the step change in load occurs, thus showing that the system states reach the desired equilibrium point. Fig. 4.17 shows the state-plane plot for the source converter obtained from the experimental data. For the experimental setup, for the source converter, the resolution for the voltage is $0.4 \mathrm{~V}$ and the resolution for current is $0.08 \mathrm{~A}$. Therefore, the output voltage can only take values at a step of $0.4 \mathrm{~V}$. Fig. 4.17 shows that the output voltage takes values of $10.4 \mathrm{~V}$ and $10.8 \mathrm{~V}$ only within the range of the surface and the input current takes values at intervals of 0.08 A. Fig. 4.19 . 4.22 shows similar state trajectories for the POLC 1. Since same step change in the load occurs for POLC 2, the state trajectories for POLC 2 are exactly similar to those of POLC 1. Fig. 4.22 shows the state-plane plot for the POLC 1 obtained from the experimental data. For the experimental setup, for the POLCs, the resolution for the voltage is $0.4 \mathrm{~V}$ and the resolution for current is $0.02 \mathrm{~A}$. Therefore, the output voltage can only take values at a step of $0.4 \mathrm{~V}$. Fig. 4.22 shows that the output voltage takes values of $20.6 \mathrm{~V}-21.4$ $\mathrm{V}$ at steps of $0.4 \mathrm{~V}$ within the range of the surface and the input current takes values at 
intervals of 0.02 A. For both the source converter and POLCs, due to the limitation in the memory resolution, the states cannot take any intermediate values. This is a limitation of this experimental set up where only 32768 memory locations could be used. This limitation can be addressed by using an EEPROM with higher number of memory locations. This will increase the memory resolution and will ensure that state trajectories take values at lesser intervals. In the simulation, the ADC was modeled with a signal delay only. Therefore, in the simulation, the signals can take continuous values. Moreover, interpolation of data takes place in the memory lookup table in the simulation. But in the experimental setup interpolation is not possible and unlike the simulation, the EEPROM reacts to sampled signals from the ADCs. This explains the deviation of the experimental results from the simulation results. The raw experimental data obtained during the transient is subjected to a moving average filter in MATLAB to generate the intermediate data points. The MATLAB in-built function smooth is used for this purpose. Fig. 4.18 and Fig. 4.23 shows the state-plane plots that use the smoothed data. The plots show that if the memory resolution is increased and the states take values at lesser intervals, then the states follow the surface more closely and reach the final equilibrium point. 


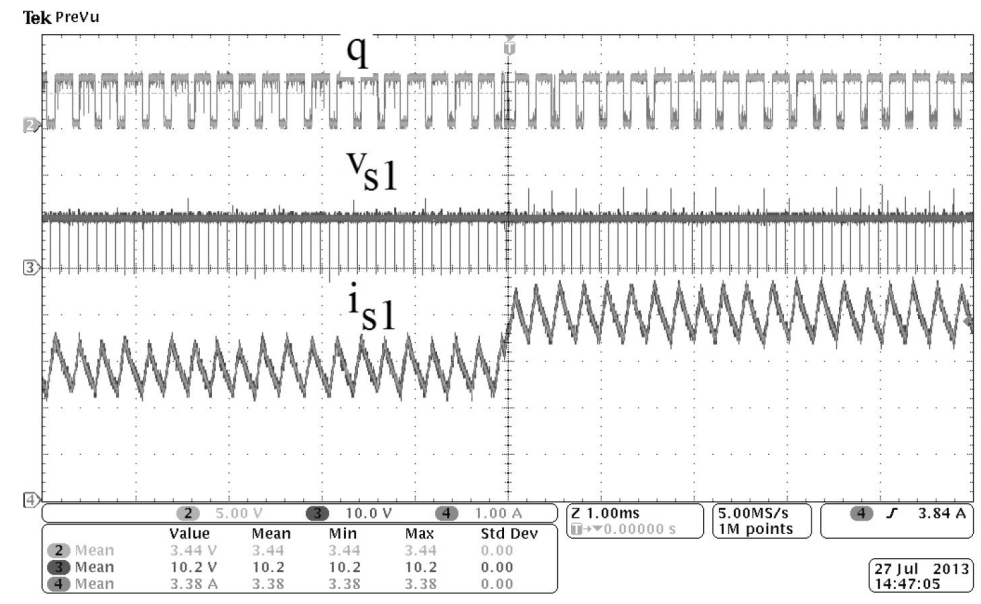

Figure 4.14: Experimental time domain plot for the source converter in the dc microgrid for a step change in load.

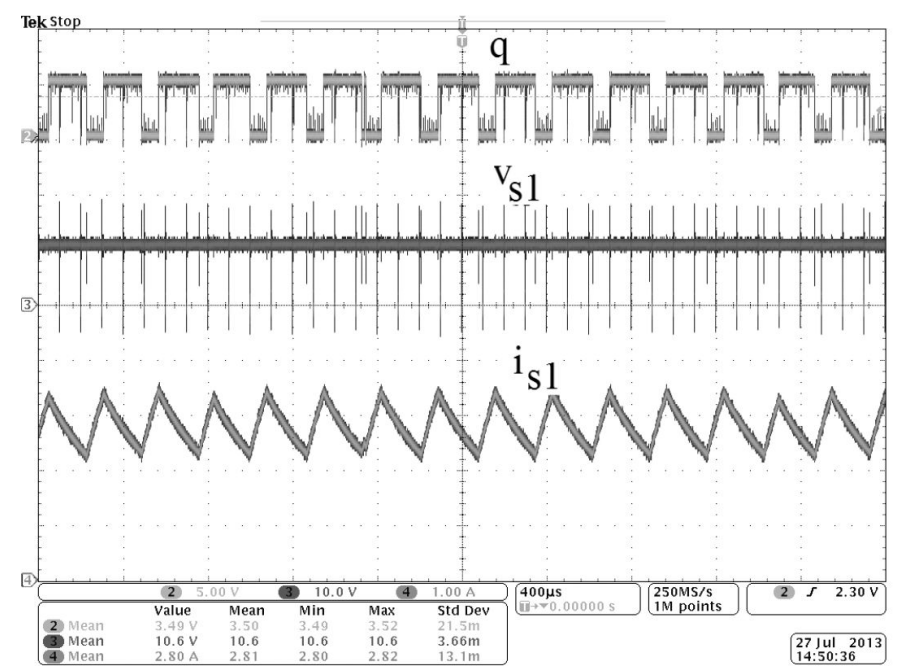

Figure 4.15: Experimental time domain plot for the source converter in the dc microgrid in the steady state before the step change in load is applied. 


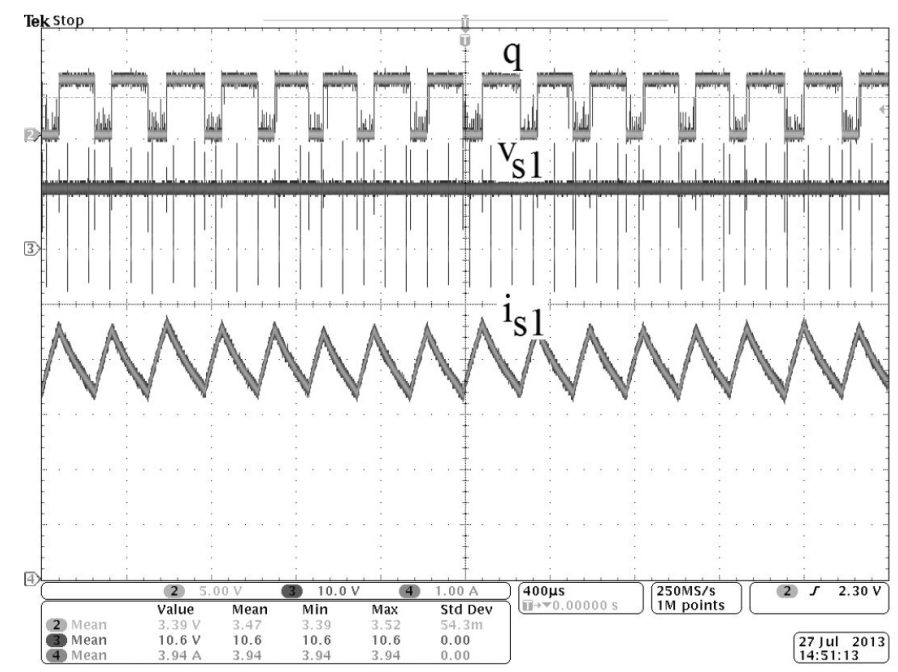

Figure 4.16: Experimental time domain plot for the source converter in the dc microgrid in the steady state after the step change in load is applied.

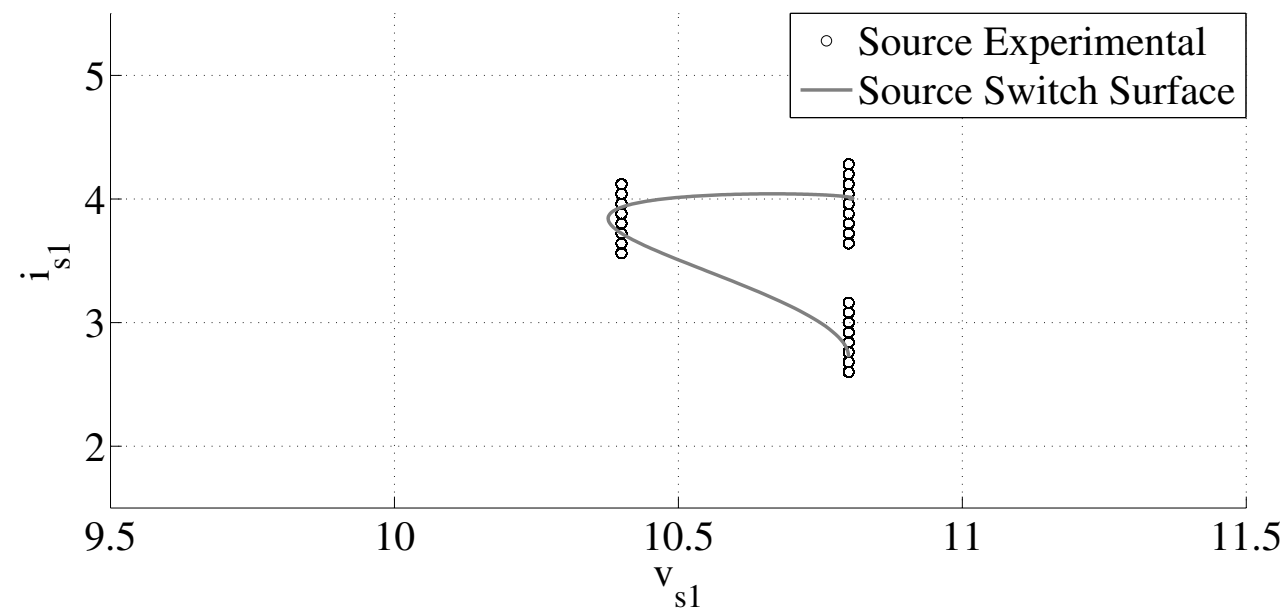

Figure 4.17: State-plane plot for the source converter in the dc microgrid for a step change in load with raw experimental data. 


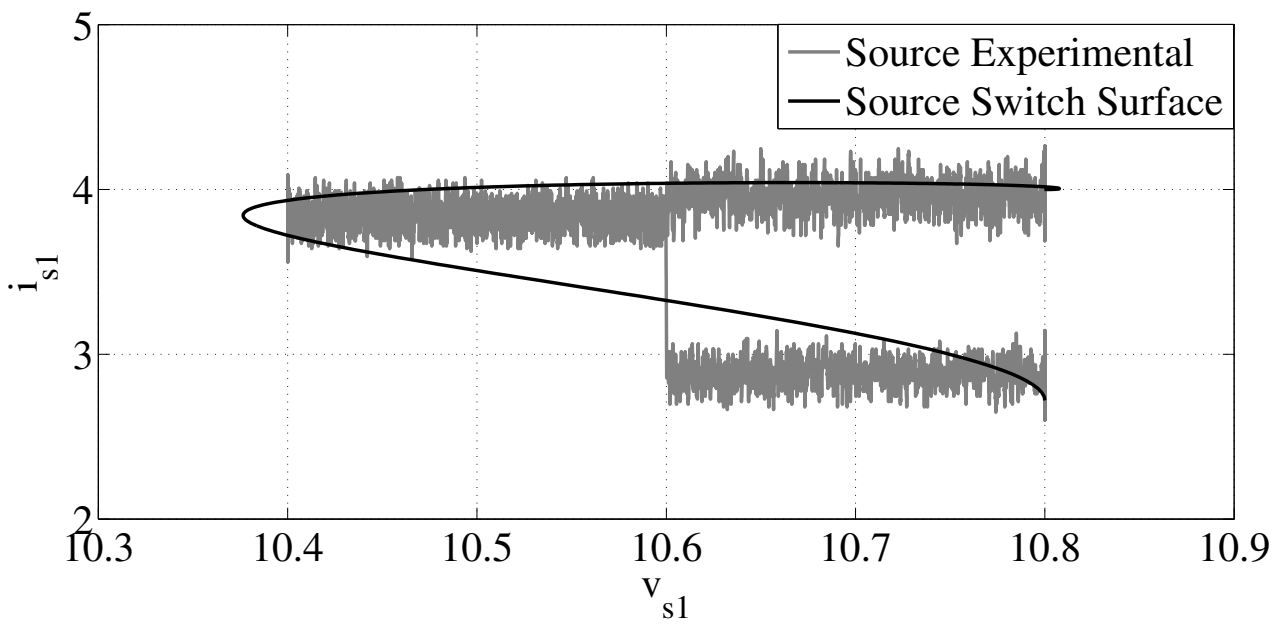

Figure 4.18: State-plane plot for the source converter in the dc microgrid for a step change in load with moving average experimental data.

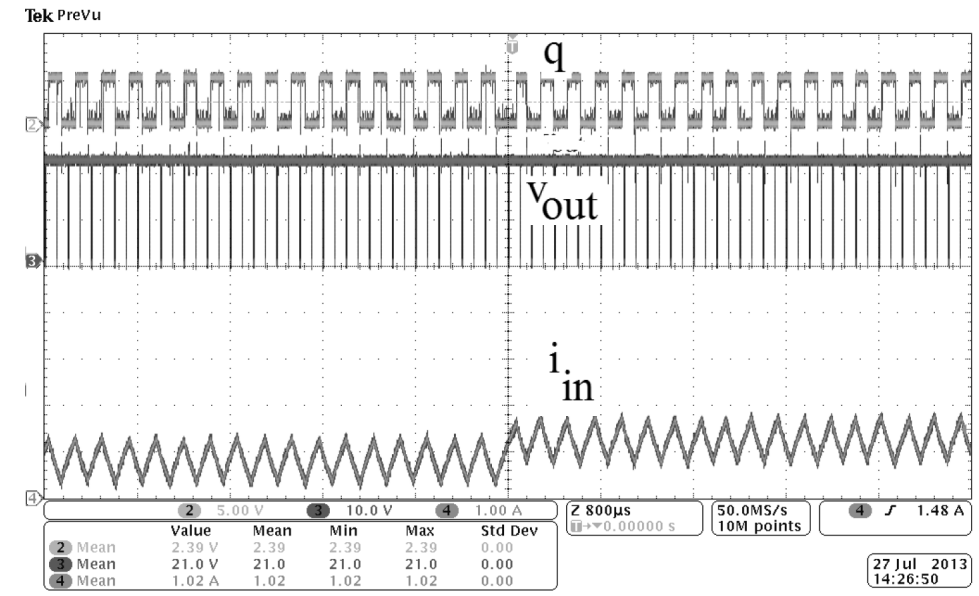

Figure 4.19: Experimental time domain plot for the POLCs in the dc microgrid for a step change in load.

Hence, the geometric surfaces can be successfully implemented as reference surfaces in the EEPROMs for a transient condition, such as step change in load and the digital sliding mode hysteretic controller could be designed effectively such that the states slide along the reference surfaces and reach the desired equilibrium points from the initial equilibrium 


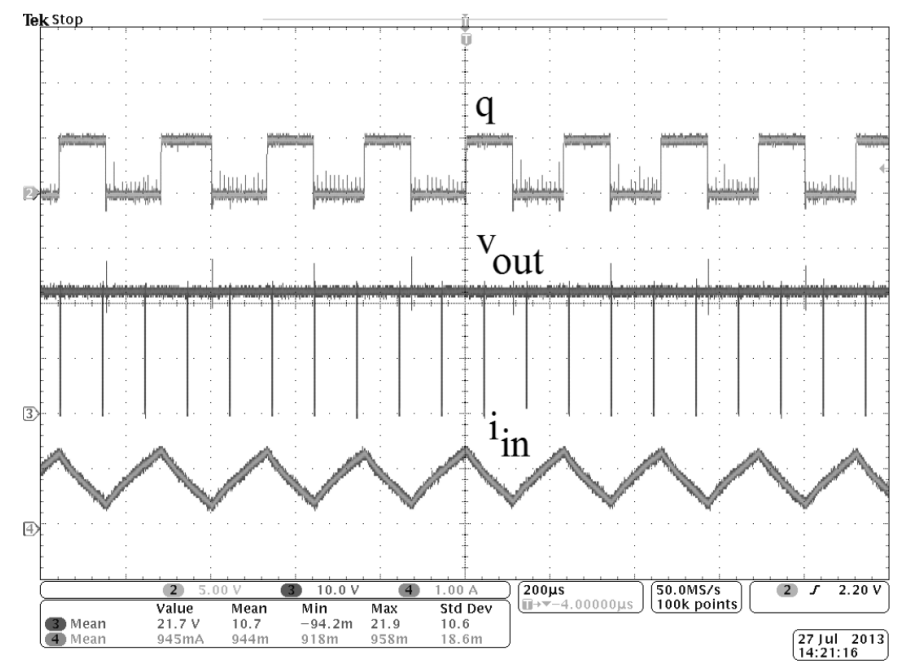

Figure 4.20: Experimental time domain plot for the POLCs in the dc microgrid in the steady state before the step change in load is applied.

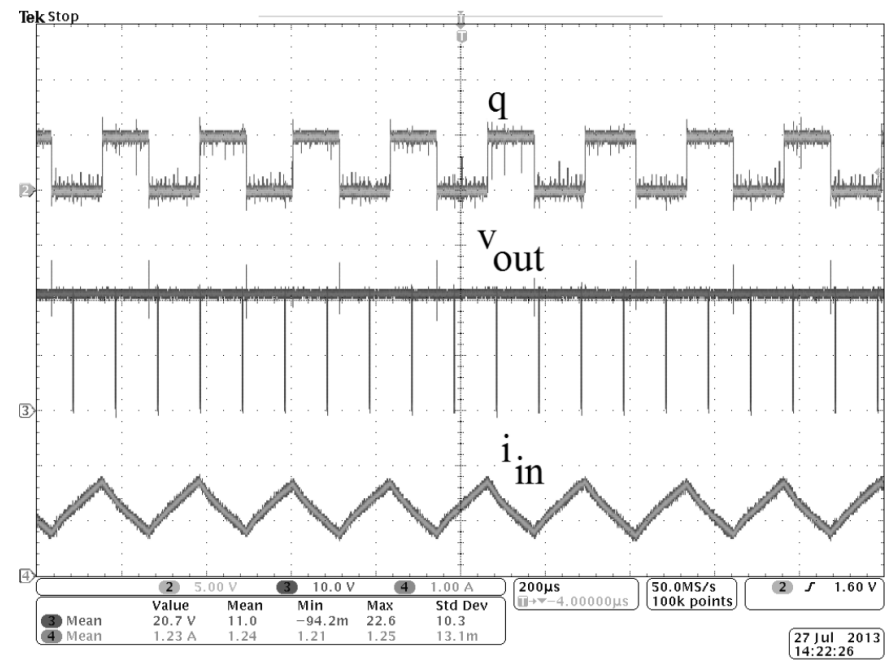

Figure 4.21: Experimental time domain plot for the POLCs in the dc microgrid in the steady state after the step change in load is applied.

points. 


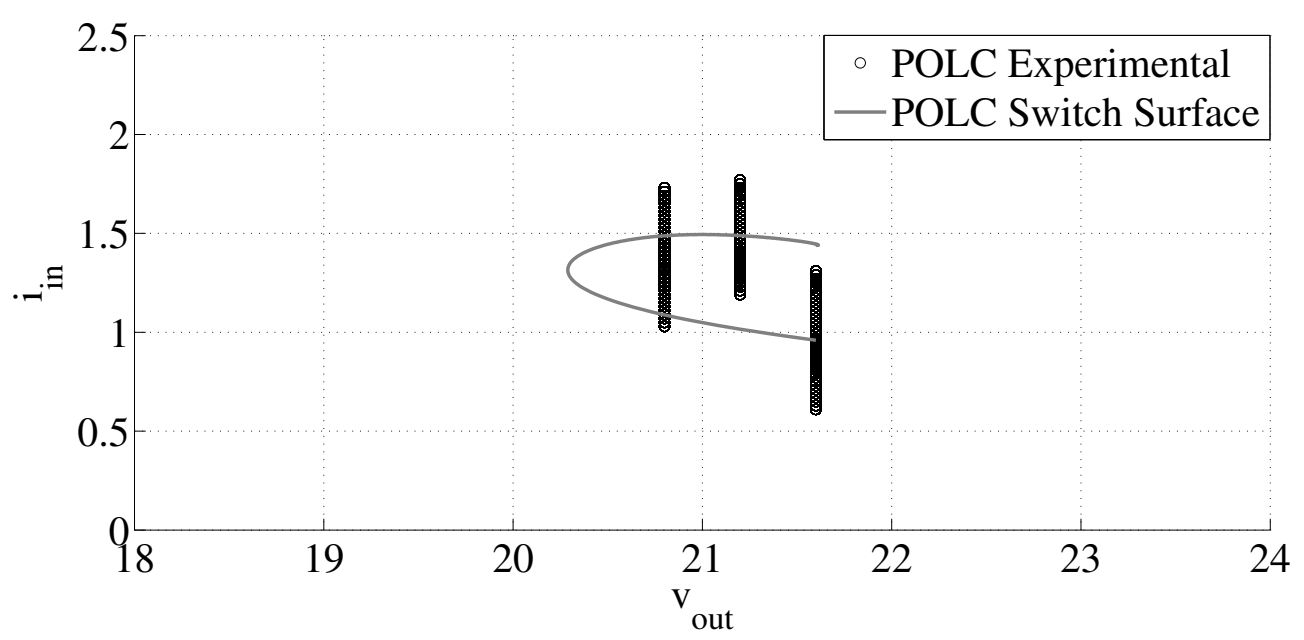

Figure 4.22: State-plane plot for the for the POLCs in the dc microgrid for a step change in load with raw experimental data.

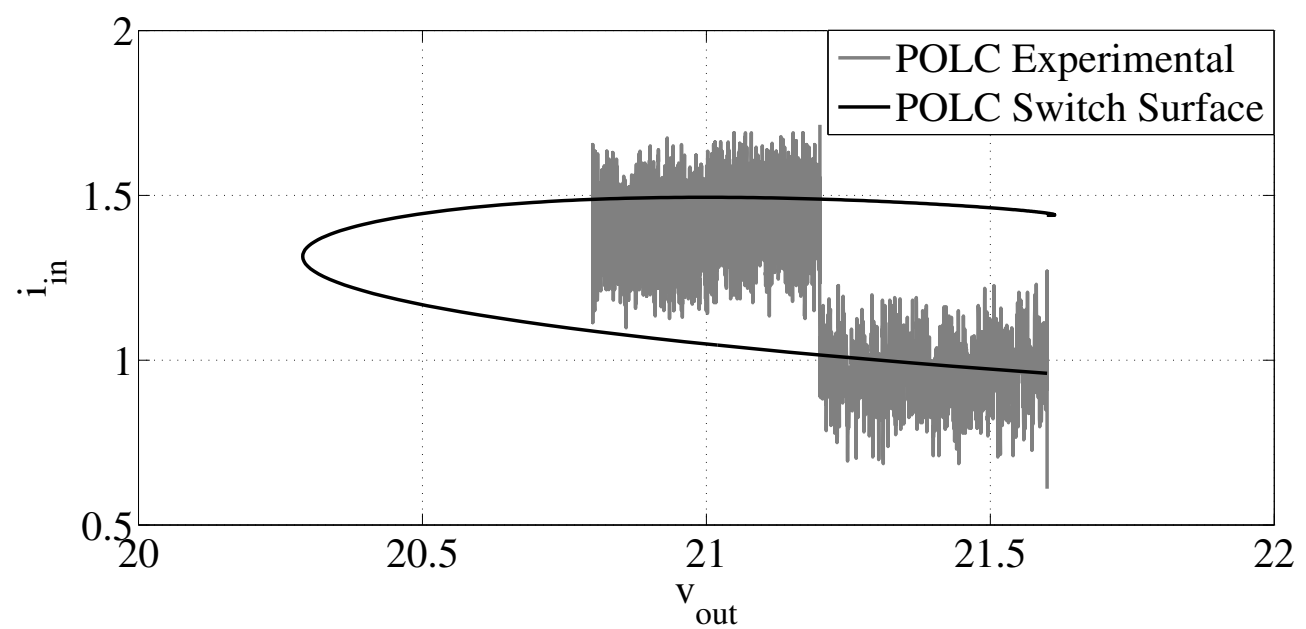

Figure 4.23: Experimental state-plane plot for the for the POLCs in the dc microgrid for a step change in load with moving average experimental data.

\subsection{Discussion}

Chapter 3 shows the application of a digital memory look-up based control strategy in a single dc-dc boost converter acting as a POLC. It is important to show that this is applicable 
for larger systems as well. The work in this chapter shows the application of the same control strategy in a dc microgrid comprising of three dc-dc boost converters. This will be applicable for systems having more than three converters as well.

The example system in this chapter is a multi-player system and the reference surface is actually derived from the solution of a multi-player game-theoretic control problem. This work actually implements a game-theoretic control trajectory in a real-time hardware set up by using it as a reference surface, thereby showing that it is possible to implement a mathematical concept, such as game-theory in a real-time hardware set up.

The stability analysis of the reference surfaces pertaining to the example system has been done to ensure the stability of the system under the chosen design conditions. This makes this control strategy an extremely robust one. If the choice of the sliding surface is such that a stability analysis proves it to be stable, then for that particular system, the sliding mode controller will prove to be a very effective and reliable controller.

\subsection{Conclusions}

The work shown in this chapter extends the work in Chapter 3 and shows that the digital sliding mode hysteretic control strategy can be implemented in a microgrid as well. The methods used in this chapter is similar to those used in Chapter 3. The complexity of the 
model increases due to the presence of three power electronic converters instead of one. For each converter, the game-theoretic control trajectories are derived for a specific case of step change in load. Those trajectories are the reference surfaces and are fed into the memory chip of the controller. This control strategy could effectively drive the system states to the reference surfaces and to the desired equilibrium points thereby validating the applicability of this strategy for larger systems. 


\section{Chapter 5}

\section{Conclusions}

\subsection{Summary of accomplishments}

The game-theoretic control trajectories for the power electronic converters in a microgrid have been derived for various events, such as step changes in the load and source. The importance of these trajectories and the complete geometric manifold they form lie in the fact that they can be used as reference surfaces for a controller. This work showed examples where the reference surfaces are used in a digital sliding mode hysteretic controller. The switch states of the power converters need to be controlled by a controller and these surfaces will act as a basis of the control. The manifolds are derived based on the specific ranges of load and source steps considered for the derivation. However, this method may be 
extended to any range of the transient events and is applicable for any number of energy storage devices in a microgrid. This method shows a general guideline for derivation of game-theoretic control based geometric manifolds in a microgrid.

The proposed strategy effectively controls the system under a normal operating condition as well as under transient conditions, such as a cold start or a sudden change in the load as evident from the simulation and experimental results obtained. It is also observed that since the real time implementation of this strategy does not require any on site mathematical calculations, the control action during a transient phenomena takes place in a very little time, thereby making this strategy really effective and fast. A memory chip being readily available and relatively much cheap as compared to any other complex controllers, make this strategy a low cost and effective control strategy in a microgrid.

The stability analysis of the reference surfaces chosen for the example systems in the dissertation shows that they ar all stable. The mathematical approach used to do the analysis will act as a general guideline for selection of sliding surfaces for power electronic converters. Although the analysis was done for some specific example cases, this method is applicable for different linear and non-linear surfaces and different power converter topologies.

The memory resolution analysis gives a guideline for the choice of the memory size of the chip to be used for the hardware of the controller. The control strategy is made simpler in terms of hardware design by eliminating the switch state feedback to the controller to 
generate hysteresis. The ADC converter signal propagation delay generates the required hysteresis band for the sliding mode controller.

The real-time hardware implementation of the control strategy provides a realistic method of implementing a highly non-linear control technique, such as sliding mode hysteretic control methodology. This also shows that open-loop mathematical solutions obtained from the game-theoretic control problem can be used in a real-time hardware set up. Hence this work shows a practical implementation of a control methodology which was limited to simulations till now.

\subsection{Recommendations for future work}

This dissertation has shown the derivation of game-theoretic geometric manifolds in a dc microgrid for transient events, such as steps in loads and sources. Many other transient events may exist in a real system, such as start up [99], series faults in the microgrid [100] , to name a few. Derivation of manifolds pertaining to many more transient events may be done as an extension of this work. The manifolds derived in Chapter 2 are based on finite event steps. No analysis has been done for the choice of the granularity in the event steps. This may be a valid analysis because it will provide a proper foundation for the number of events to be considered for the derivation of the manifolds. 
Pontryagin's minimum principle is used to solve the game-theoretic control problem in Chapter 2, This method yields open form solutions. These solutions do not have any proper mathematical form that can be directly used in a controller. One important area to explore is to use a Hamilton-Jacobi-Bellman (HJB) equation [86] to give a closed form solution to the problem under consideration. This will help in the design of a feedback controller that will use the closed form solutions to react to the transient events in the system.

The game-theoretic control method used in this work is based on a non-cooperative differential game [101]. Here the players are unaware of the decisions of the other players. It just tries to meet its own objective based only on the local information that is available to it. The performance of the whole system can be improved by establishing some form of communication between the players. The decision of a player will new be dependent on the decision of other players in the game. This is the basis of a cooperative differential game [102].

Since the solution of the game-theoretic control problem is based on solving complex differential equations, different methods of solving the problem may be explored. In this dissertation, the minimum principle method is solved using the bvp $4 \mathrm{c}$ command in MATLAB. Different methods, such as shooting, collocation, finite difference, [103] etc. may be used to solve this two-point boundary value problem. Also, the solution process may be simplified by reducing the system order to achieve a simple solution.

Further research into real-time implementation of the digital sliding mode hysteretic control 
technique needs to explore the memory resolution effects of the digital controller in more details. In this work the limits of the memory size has not been explicitly derived. There is scope to formulate a mathematical formula that would explicitly specify the upper and the lower limits of the memory resolution for a particular system. Similar limits for the ADC latency may also be derived and the relationship between the ADC latency and the hysteresis band may be mathematically formulated for a given system.

This dissertation has given a general guideline of generating game-theoretic geometric manifolds in a microgrid for various transient events. A method of real-time implementation of these manifolds in a controller has been shown in this work. This research will continue to explore the application of game theory to the control of power electronic devices and will be a guideline for hardware implementation of different non-linear control methodologies in a power electronics based system. 



\section{References}

[1] B. Banerjee and W. Weaver, "Geometric manifold control of power electronics in dc microgrids," in Proceedings of the IEEE Workshop on Control and Modeling for Power Electronics, pp. 1-8, june 2012.

[2] H. B. Puttgen, P. MacGregor, and F. Lambert, "Distributed generation: Semantic hype or the dawn of a new era?," IEEE Power and Energy Magazine, vol. 1, no. 1, pp. 22-29, 2003.

[3] W. Zhang, H. Liang, Z. Bin, W. Li, and R. Guo, "Review of dc technology in future smart distribution grid," in Proceedings of the IEEE Innovative Smart Grid Technologies - Asia, pp. 1-4, 2012.

[4] J. Liu, W. Zhang, R. Zhou, and J. Zhong, "Impacts of distributed renewable energy generations on smart grid operation and dispatch," in Proceedings of the IEEE Power and Energy Society General Meeting, pp. 1-5, 2012. 
[5] W. Tushar, W. Saad, H. V. Poor, and D. B. Smith, "Economics of electric vehicle charging: A game theoretic approach," IEEE Transactions on Smart Grid, vol. 3, no. 4, pp. 1767-1778, 2012.

[6] M. C. Bozchalui, S. A. Hashmi, H. Hassen, C. A. Canizares, and K. Bhattacharya, "Optimal operation of residential energy hubs in smart grids," IEEE Transactions on Smart Grid, vol. 3, no. 4, pp. 1755-1766, 2012.

[7] M. J. Osborne and A. Rubinstein, A Course in game Theory. Binghamton, NY: The Maple-Vail Book Manufacturing Group, 1 ed., 1994.

[8] Z. M. Fadlullah, Y. Nozaki, A. Takeuchi, and N. Kato, "A survey of game theoretic approaches in smart grid," in Proceedings of the International Conference on Wireless Communications and Signal Processing, pp. 1-4, 2011.

[9] A. Mohsenian-Rad, V. W. S. Wong, J. Jatskevich, R. Schober, and A. Leon-Garcia, "Autonomous demand-side management based on game-theoretic energy consumption scheduling for the futire smart grid," IEEE Transactions on Smart Grid, vol. 1, no. 3, pp. 320-331, 2010.

[10] T. Agarwal and S. Cui, "Noncooperative games for autonomous consumer load balancing over smart grid," http://arxiv.org/abs/1104.3802, 2011.

[11] P. Aristidou, A. Dimeas, and N. Hatziargyriou, "Microgrid modeling and analysis using game theory methods," Energy-Efficient Computing and Networking, 
Lecture Notes of the Institute for Computer Sciences, Social Informatics, and Telecommunications Engineering, vol. 54, no. 1, pp. 12-19, 2011.

[12] M. Alamaniotis, R. Gao, and L. Tsoukalas, "Towards an energy internet: A game-theoretic approach to price-directed energy utilization," in Proceedings of the International ICST Conference on E-Energy, pp. 3-10, 2010.

[13] P. Vytelingum, T. D. Voice, S. D. Ramchurn, A. Rogers, and N. R. Jennings, "Agent-based micro-storage management for the smart grid," in Proceedings of the International Conference on Autonomous Agents and Multiagent Systems, vol. 1, 2010.

[14] C. Ibars, M. Navarro, and L. Giupponi, "Distributed demand management in smart grid with a congestion game," in Proceedings of the IEEE International Conference on Smart Grid Communications, pp. 495-500, 2010.

[15] A. Ball, M. Lim, D. Gilham, and F. C. Lee, "System design of a 3d integrated non-isolated point of load converter," in Proceedings of the IEEE Applied Power Electronics Conference and Exposition, pp. 181-186, feb. 2008.

[16] R. D. Middlebrook and S. Cuk, "A general unified approach to modeling switching-converter power stages," in Proceedings of the IEEE Power Electronics Specialists Conference, pp. 73-86, june 1976. 
[17] W. W. Weaver and P. T. Krein, "Distributed dynamic energy resource control," in Proceedings of the IEEE Electric Ship Technologies Symposium, pp. 182 -188, april 2009.

[18] W. Weaver and P. Krein, "Optimal geometric control of power buffers," IEEE Transactions on Power Electronics, vol. 24, pp. 1248 -1258, May 2009.

[19] G. M. Vosters and W. W. Weaver, "Energy and impedance space modeling of power electronic converters," in Proceedings of the IEEE Energy Conversion Congress and Exposition, pp. 1265 -1272, sept. 2011.

[20] R. S. Balog, W. W. Weaver, and P. T. Krein, "The load as an energy asset in a distributed architecture," in Proceedings of the IEEE Electric Ship Technologies Symposium, pp. 261 - 267, july 2005.

[21] J. J. Grainger and W. D. Stevenson, Power System Analysis. McGraw-Hill Education (India) Pvt Limited, 2003.

[22] X. Liu, P. Wang, and P. C. Loh, "A hybrid ac/dc microgrid and its coordination control," IEEE Transactions on Smart Grid, vol. 2, no. 2, pp. 278-286, 2011.

[23] H. Guldemir, "Sliding mode control of dc-dc boost converter," Journal of Applied Sciences, vol. 5, no. 3, pp. 588 -592, 2005. 
[24] J. H. Su, J. J. Chen, and D. S. Wu, "Learning feedback controller design of switching converters via matlab/simulink," IEEE Transactions on Education, vol. 45, pp. 307 -315 , Nov 2002.

[25] P. Mattavelli, L. Rossetto, and G. Spiazzi, "Small-signal analysis of dc-dc converters with sliding mode control," in Proceedings of Applied Power Electronics Conference and Exposition, vol. 1, pp. 153 -159, Mar 1995.

[26] Y. Tarte, Y.-Q. Chen, W. Ren, and K. Moore, "Fractional horsepower dynamometer a general purpose hardware-in-the-loop real-time simulation platform for nonlinear control research and education," in Proceedings of the IEEE Conference on Decision and Control, pp. 3912-3917, 2006.

[27] J. Alvarez-Ramirez, G. Espinosa-Perez, and D. Noriega-Pineda, "Current-mode control of dc-dc power converters: a backstepping approach," in Proceedings of the IEEE International Conference on Control Applications, pp. 190 -195, 2001.

[28] H. Guldemir, "Study of sliding mode control of dc-dc buck converter," Journal of Energy and Power Engineering, vol. 3, pp. 401 -406, 2011.

[29] Y. B. Shtessel, A. S. I. Zinober, and I. A. Shkolnikov, "Boost and buck-boost power converters control via sliding modes using dynamic sliding manifold," in Proceedings of the Conference on Decision and Control, vol. 3, pp. 2456-2461, dec. 2002 . 
[30] H. Guldemir, "Sliding mode control of dc-dc boost converter," Journal of Applied Sciences, vol. 5, no. 3, pp. $588-592,2005$.

[31] P. Mattavelli, L. Rossetto, and G. Spiazzi, "Small-signal analysis of dc-dc converters with sliding mode control," in Proceedings of Applied Power Electronics Conference and Exposition, vol. 1, pp. 153 -159, Mar 1995.

[32] Y. He and F. Luo, "Sliding-mode control for dc-dc converters with constant switching frequency," Control Theory and Applications, vol. 153, pp. 37 - 45, Jan. 2006.

[33] Y. F. Liu and L. Jia, "Performance enhancement with digital control technologies for dc-dc switching converters," in Proceedings of the IEEE Control and Modeling for Power Electronics, pp. 1 -8, june 2010.

[34] G. Pitel and P. Krein, "Minimum-time transient recovery for dc-dc converters using raster control surfaces," IEEE Transactions on Power Electronics, vol. 24, pp. 2692 -2703, Dec. 2009.

[35] D. Gan, F. Liu, L. Du, and Y. Liu, "Research and implementation of on-line monitoring techniques for high voltage equipments in smart grid," in Proceedings of the International Conference on High Voltage Engineering and Application, pp. 236-239, 2010. 
[36] G. Reed, P. Philip, A. Barchowsky, C. Lippert, and A. Sparacino, "Sample survey of smart grid approaches and technology gap analysis," in Proceedings of the IEEE PES Innovative Smart Grid Technologies Conference Europe, pp. 1-10, 2010.

[37] Q. Chen, C. Kang, Q. Xia, and J. Zhong, "Power generation expansion planning model towards low-carbon economy and its application in china," IEEE Transactions on Power Systems, vol. 25, no. 2, pp. 1117-1125, 2010.

[38] M. Barnes, J. Kondoh, H. Asano, J. Oyarzabal, G. Venkataramanan, R. Lasseter, N. Hatziargyriou, and T. Green, "Real-world microgrid- an overview," in Proceedings of the IEEE International Conference on System of Systems Engineering, pp. 1 -8, april 2007.

[39] F. Katiraei and M. R. Iravani, "Power management strategies for a microgrid with multiple distributed generation units," IEEE Transactions on Power Systems, vol. 21, pp. $1821-1831$, nov. 2006.

[40] P. Basak, A. Saha, C. S., and C. S. P., "Microgrid: Control techniques and modeling," in Proceedings of the International Universities Power Engineering Conference, pp. $1-5$, sept. 2009.

[41] J. Martin, "Distributed vs. centralized electricity generation: are we witnessing a change of paradigm?," http://www.vernimmen.net/ftp/An-introduction-to-distribu ted-generation.pdf, 2012. 
[42] G. Pepermansa, J. Driesenb, D. Haeseldonckxc, R. Belmansc, and W. D’haeseleerc, "Distributed generation: definition, benefits and issues," Energy Policy, vol. 33, pp. 787-798, 2005.

[43] W. Yang, A. Xin, and G. Yang, “Microgrid's operation-management containing distributed generation system," in Proceedings of the International Conference on Electric Utility Deregulation and Restructuring and Power Technologies, pp. 703-707, july 2011.

[44] F. Farret and M. Simőes, Integration of Alternative Sources of Energy. Wiley-IEEE Press, 1 ed., 2006.

[45] Y.-D. Lee, Y.-R. Chang, C.-M. Chan, and Y.-H. Ho, "Preliminary implementation of microgrid with photovoltaic and microturbine for stand alone operation," in Proceedings of the IEEE Industry Applications Society Annual Meeting, pp. 1-9, 2012.

[46] Z. Wang, X. Huang, and J. Jiang, "Design and implementation of a control system for a microgrid involving a fuel cell power module," in Proceedings of the IEEE Electrical Power Conference, pp. 207-212, 2007.

[47] R. H. Lasseter and P. Paigi, "Microgrid: A conceptual solution," in Proceedings of the IEEE Power Electronics Specialists Conference, pp. 4285 -4290, 2004.

[48] P. Biczel, "Power electronic converters in dc microgrid," in Proceedings of the Compatibility in Power Electronics, pp. 1 -6, 2007. 
[49] F. Katiraei, M. Iravani, and P. Lehn, "Microgrid autonomous operation during and subsequent to islanding process," in Proceedings of the IEEE Power Engineering Society General Meeting, pp. 2175 Vol.2-, 2004.

[50] M. Barnes, J. Kondoh, H. Asano, J. Oyarzabal, G. Ventakaramanan, R. Lasseter, N. Hatziargyriou, and T. Green, "Real-world microgrids-an overview," in Proceedings of the IEEE International Conference on System of Systems Engineering, pp. 1-8, 2007.

[51] Y. Ito, Y. Zhongqing, and H. Akagi, "Dc microgrid based distribution power generation system," in Proceedings of the International Power Electronics and Motion Control Conference, vol. 3, pp. 1740-1745 Vol.3, 2004.

[52] H. Kakigano, M. Nomura, and T. Ise, "Loss evaluation of dc distribution for residential houses compared with ac system," in Proceedings of the International Power Electronics Conference, pp. 480 -486, 2010.

[53] H. Kakigano, Y. Miura, T. Ise, and R. Uchida, "Dc voltage control of the dc micro-grid for super high quality distribution," in Proceedings of the Power Conversion Conference, pp. 518-525, april 2007.

[54] H. Kakigano, Y. Miura, and T. Ise, "Low-voltage bipolar-type dc microgrid for super high quality distribution," IEEE Transactions on Power Electronics, vol. 25, pp. 3066-3075, dec. 2010. 
[55] M. D. Davis, Game Theory A Non Technical Introduction. Mineola, NY: Dover Publications, 1 ed., 1997.

[56] M. Athans and P. L. Falb, Optimal Control. New York: McGraw-Hill, 1966.

[57] W. Weaver, "Dynamic energy resource control of power electronics in local area power networks," IEEE Transactions on Power Electronics, vol. 26, no. 3, pp. 852-859, 2011.

[58] T. Başar and G. J. Olsder, Dynamic Noncooperative Game Theory. Philadelphia,PA: SIAM, 2 ed., 1999.

[59] G. Owen, Game Theory. New York, NY: Academic Press, 3 ed., 2001.

[60] R. Isaacs, Differential Games: A Mathematical Theory with Applications to Warfare and Pursuit, Control and Optimization. New York, NY: John Wiley and Sons, 1 ed., 1965.

[61] T. Alpcan, L. Pavel, and N. Stefanovic, "A control theoretic approach to noncooperative game design," in Proceedings of the IEEE Conference on Decision and Control, pp. 8575-8580, 2009.

[62] E. Altman, T. Boulogne, R. El-Azouzi, T. Jiménez, and L. Wynter, “A survey on networking games in telecommunications," Computers and Operations Research, vol. 33, no. 2, pp. 286-311, 2006. 
[63] L. E. Li, J. Y. Halpern, P. Bahl, Y.-M. Wang, and R. Wattenhofer, "A cone-based distributed topology-control algorithm for wireless multi-hop networks," Transactions on Networking, vol. 13, no. 1, pp. 147-159, 2005.

[64] P. Parag, S. Shakkottai, and J.-F. Chamberland, "Value-aware resource allocation for service guarantees in networks," in Proceedings of the IEEE International Conference on Computer Communications, 2010.

[65] Y. Su and M. van der Schaar, "A new perspective on multi-user power control games in interference channels," Transactions on Wireless Communications, vol. 8, no. 6, pp. 2910-2919, 2009.

[66] R. Gopalakrishnan, J. R. Marden, and A. Wierman, "An architectural view of game theoretic control," ACM SIGMETRICS Performance Evaluation Review, vol. 38, no. 3,2010 .

[67] V. Utkin, "Sliding modes in multidimensional systems with variable structure," in Proceedings of the IEEE Conference on Decision and Control including the 12th Symposium on Adaptive Processes, vol. 12, pp. 727-727, 1973.

[68] J. Yang, G. He, and J. Cui, “Analysis of pmlsm direct thrust control system based on sliding mode variable structure," in Proceedings of the CES/IEEE International Power Electronics and Motion Control Conference, vol. 1, pp. 1-5, 2006.

[69] H. K. Khalil, Nonlinear Systems. Upper Saddle River, NJ: Prentice-Hall, 3 ed., 2002. 
[70] C.-H. Hsu, T.-W. Chang, and C.-L. Wey, "A voltage-mode hysteretic boost dc-dc converter with dual control modes," in Proceedings of the Asia Pacific Conference on Postgraduate Research in Microelectronics and Electronics, pp. 67-71, 2012.

[71] W. Perruquetti and J. P. Barbot, eds., Sliding Mode Control in Engineering. Madison Avenue, NY: Marcel Dekker Inc., 2002.

[72] H. Sira-Ramirez, "Sliding motions in bilinear switched networks," IEEE Transactions on Circuits and Systems, vol. 34, no. 8, pp. 919-933, 1987.

[73] H. Sira-Ramirez, "Nonlinear variable structure systems in sliding mode: the general case," IEEE Transactions on Automatic Control, vol. 34, no. 11, pp. 1186-1188, 1989.

[74] Y. Li, Y. Chen, and I. Podlubny, "Stability of fractional-order nonlinear dynamic systems: Lyapunov direct method and generalized mittag-leffler stability," Computers and Mathematics with Applications, vol. 59, pp. 1810-1821, 2010.

[75] S. A. Bock, J. Pinheiro, H. Grundling, H. Hey, and H. Pinheiro, "Existence and stability of sliding modes in bi-directional dc-dc converters," in Proceedings of the IEEE Power Electronics Specialists Conference, vol. 3, pp. 1277-1282, 2001.

[76] C. Edwards and S. Spurgeon, Sliding Mode control: Theory and Applications. London: Taylor and Francis, 1998. 
[77] J. Carrasco, J. M. Quero, and L. Franquelo, "Multi-input variable structure controller for electronic power converters implemented by neural network techniques," in Proceedings of the IEEE Power Electronics Specialists Conference, vol. 2, pp. 1346-1351 vol.2, 1995.

[78] P. Iwanciw, "Applications of microprocessor controlled inverter drives," in Proceedings of the International Conference on Power Electronics and Variable-Speed Drives, pp. 202-205, 1988.

[79] J. Pontt, J. Rodriguez, and R. Huerta, "Digital signal processing course innovations for power electronics practice," in Proceedings of the Frontiers in Education, vol. 2, pp. F1B-6-11 Vol.2, 2003.

[80] J. S. Chitode, Power Electronics. Pune, India: Technical Publications, 4 ed., 2009.

[81] D. Maksimovic, R. Zane, and R. Erickson, "Impact of digital control in power electronics," in Proceedings of the International Symposium on Power Semiconductor Devices and ICs, pp. 13-22, 2004.

[82] ATMEL, 256K (32K x 8) Paged Parallel EEPROM (AT28C256), 2009.

[83] B. P. Lathi, Modern Digital and Analog Communication Systems. Oxford University Press, 3 ed., 1998. 
[84] J. M. Pereira, O. Postolache, F. Dengchao, V. Viegas, and P. Girao, “Advantages of pwm-a/d conversion techniques in smart sensing systems," in Proceedings of the Conference on Electronic Measurements and Instruments, pp. 5-10, july 2007.

[85] C. Q. Lee and K. Siri, "Analysis and design of series resonant converter by state-plane diagram," IEEE Transactions on Aerospace and Electronic Systems, vol. AES-22, no. 6, pp. 757-763, 1986.

[86] A. Sage and C. C. White, Optimum Systems Control. Englewood Cliffs, NJ: Prentice-Hall, second ed., 1977.

[87] E. Vidal-Idiarte, C. Carrejo, J. Calvente, and L. Marti andnez Salamero, "Two-loop digital sliding mode control of dc-dc power converters based on predictive interpolation," IEEE Transactions on Industrial Electronics, vol. 58, pp. 2491 -2501, june 2011.

[88] R. Aghatehrani and R. Kavasseri, "Sliding mode control approach for voltage regulation in microgrids with dfig based wind generations," in Proceedings of the IEEE Power and Energy Society General Meeting, pp. 1 -8, july 2011.

[89] B. Labbe, B. Allard, X. Lin-Shi, and D. Chesneau, "An integrated sliding-mode buck converter with switching frequency control for battery-powered applications," IEEE Transactions on Power Electronics, vol. 28, no. 9, pp. 4318-4326, 2013.

[90] S. Gupta and A. Phatak, "Adc guide, part 1: the ideal adc," http://www.edn.com/contenteetimes/documents/schweber/c0895/c0895pt1.pdf. 
[91] W. Kester, "Understand SINAD, ENOB, SNR, THD, THD+N, and SFDR so You Don't Get Lost in the Noise Floor," http://www.analog.com/static/imported-files/tutorials/MT-003.pdf.

[92] B. Kumari, R. S. Rana, and C. Y. Patil, "Fpga and dspace based sliding mode control of boost converter for pem fuel cell application," International Journal of Computer Applications, vol. 51, no. 18, pp. 4-10, 2012.

[93] J. Q. Borrás, "Mcu controlled dc-dc buck/boost converter for super capacitors," Master's thesis, KTH Royal Institute of Technology, Stockholm, Sweden, 62012.

[94] N. Gupta and S. P. S. S. P. Dubey, "Dsp based adaptive hysteresis-band current controlled active filter for power quality conditioning under non-sinusoidal supply voltages," International Journal of Engineering, Science and Technology, vol. 3, no. 4, pp. 236-252, 2011.

[95] http://www.digikey.com/.

[96] https://estore.ti.com/TMDSCNCD28335-TMS320F28335-controlCARD-P1309.aspx.

[97] N.H.Kutkut, C. Q. Lee, and I. Batarseh, "A generalized program for extracting the control characteristics of resonant converters via the state-plane diagram," in Proceedings of the IEEE Power Electronics Specialists Conference, vol. 2, pp. 1236-1242 vol.2, 1995. 
[98] T. Habetler and R. Harley, "Power electronic converter and system control," Proceedings of the IEEE, vol. 89, pp. 913 -925, Jun 2001.

[99] N. C. Ekneligoda and W. W. Weaver, "Optimal transient control of microgrids using a game theoretic approach," in Proceedings of the IEEE Energy Conversion Congress and Exposition, pp. 935-942, 2011.

[100] F. M. Uriarte, H. B. Estes, T. J. Hotz, A. L. Gattozzi, J. D. Herbst, A. Kwasinski, and R. E. Hebner, "Development of a series fault model for dc microgrids," in Proceedings of the IEEE Innovative Smart Grid Technologies, pp. 1-8, 2012.

[101] R. Srikant and T. Basar, "Iterative computation of noncooperative equilibria in nonzero-sum differential games with weakly coupled players," in Proceedings of the IEEE Conference on Decision and Control, pp. 252-257 vol.1, 1990.

[102] A. Haurie, B. Tolwinski, and G. Leitmann, "Cooperative equilibria in differential games," in Proceedings of the American Control Conference, pp. 1345-1350, 1983.

[103] R. W. Holsapple, R. Venkataraman, and D. Doman, "New, fast numerical method for solving two-point boundary-value problems," Journal of Guidance, Control, and Dynamics, vol. 27, no. 2, pp. 301-304, 2004.

[104] J. Alvarez-Ramirez, G. Espinosa-Perez, and D. Noriega-Pineda, "Current-mode control of dc-dc power converters: a backstepping approach," in Proceedings of the IEEE International Conference on Control Applications, pp. 190 -195, 2001. 
[105] H. Guldemir, "Study of sliding mode control of dc-dc buck converter," Journal of Energy and Power Engineering, vol. 3, pp. 401 -406, 2011.

[106] Y. He and F. Luo, "Sliding-mode control for dc-dc converters with constant switching frequency," Control Theory and Applications, vol. 153, pp. 37 - 45, Jan. 2006.

[107] P. Krein, J. Bentsman, R. Bass, and B. Lesieutre, "On the use of averaging for the analysis of power electronic systems," IEEE Transactions on Power Electronics, vol. 5, pp. 182 -190, Apr. 1990.

[108] W. W. Weaver and P. T. Krein, "Game-theoretic control of small-scale power systems," IEEE Transactions on Power Delivery, vol. 24, pp. 1560 -1567, july 2009.

[109] J. H. Su, J. J. Chen, and D. S. Wu, "Learning feedback controller design of switching converters via matlab/simulink," IEEE Transactions on Education, vol. 45, pp. 307 -315 , Nov 2002.

[110] T. Siew-Chong, Y. Lai, and C. Tse, "Implementation of pulse-width-modulation based sliding mode controller for boost converters," IEEE Power Electronics Letters, vol. 3 , pp. $130-135$, dec. 2005 .

[111] S. Rapuano, P. Daponte, E. Balestrieri, L. De Vito, S. Tilden, S. Max, and J. Blair, "Adc parameters and characteristics," IEEE Instrumentation Measurement Magazine, vol. 8, pp. 44 - 54, dec. 2005. 
[112] K. Viswanathan, R. Oruganti, and D. Srinivasan, "Non-linear function controller: a simple alternative to fuzzy logic controller for a power electronic converter," in Proceedings of the Conference of IEEE Industrial Electronics Society, vol. 3, pp. 2655-2660, nov. 2004.

[113] V. Utkin and H. Lee, "Chattering problem in sliding mode control systems," in Proceedings of the International Workshop on Variable Structure Systems, pp. 346-350, 2006.

[114] L. Encarnacao and J. Silva, "Stability condition based sliding mode modulators for multilevel power converters," in Proceedings of the IEEE Conference of Industrial Electronics, pp. 877-882, 2009. 


\section{Appendix A}

\section{MATLAB scripts and Simulink models}

\section{A.1 MATLAB script for the solution of game-theoretic control BVP for the de microgrid}

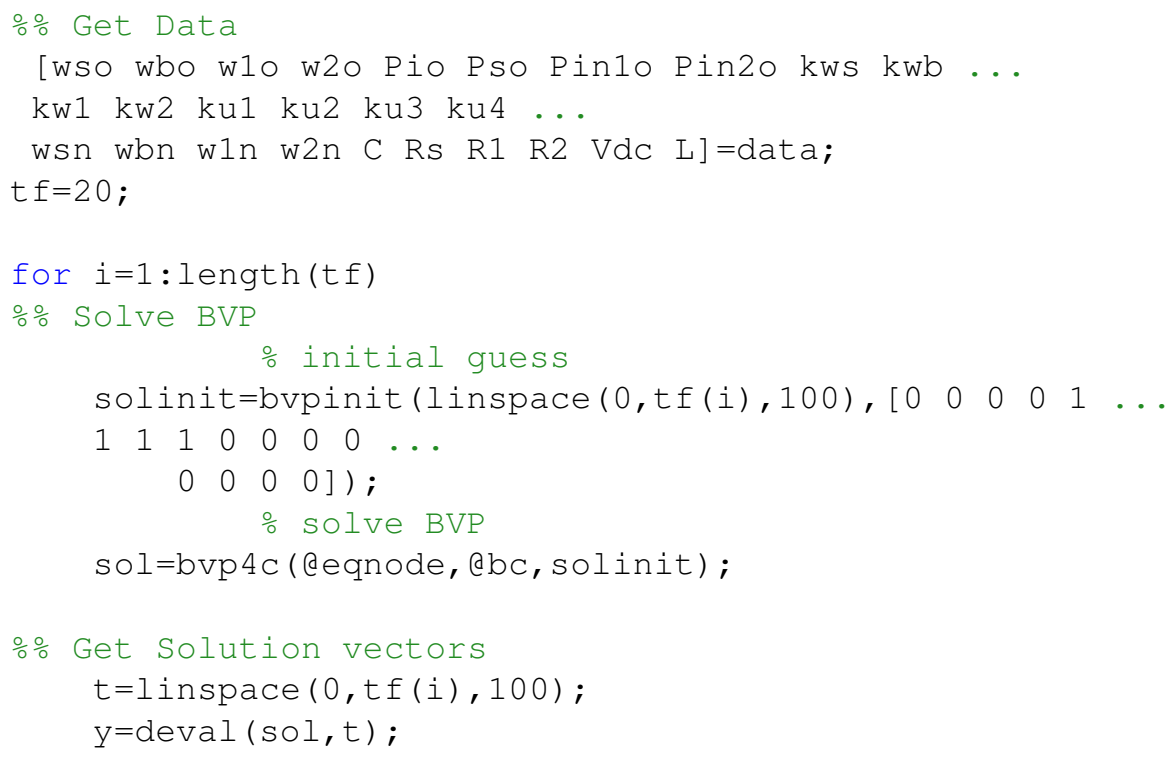




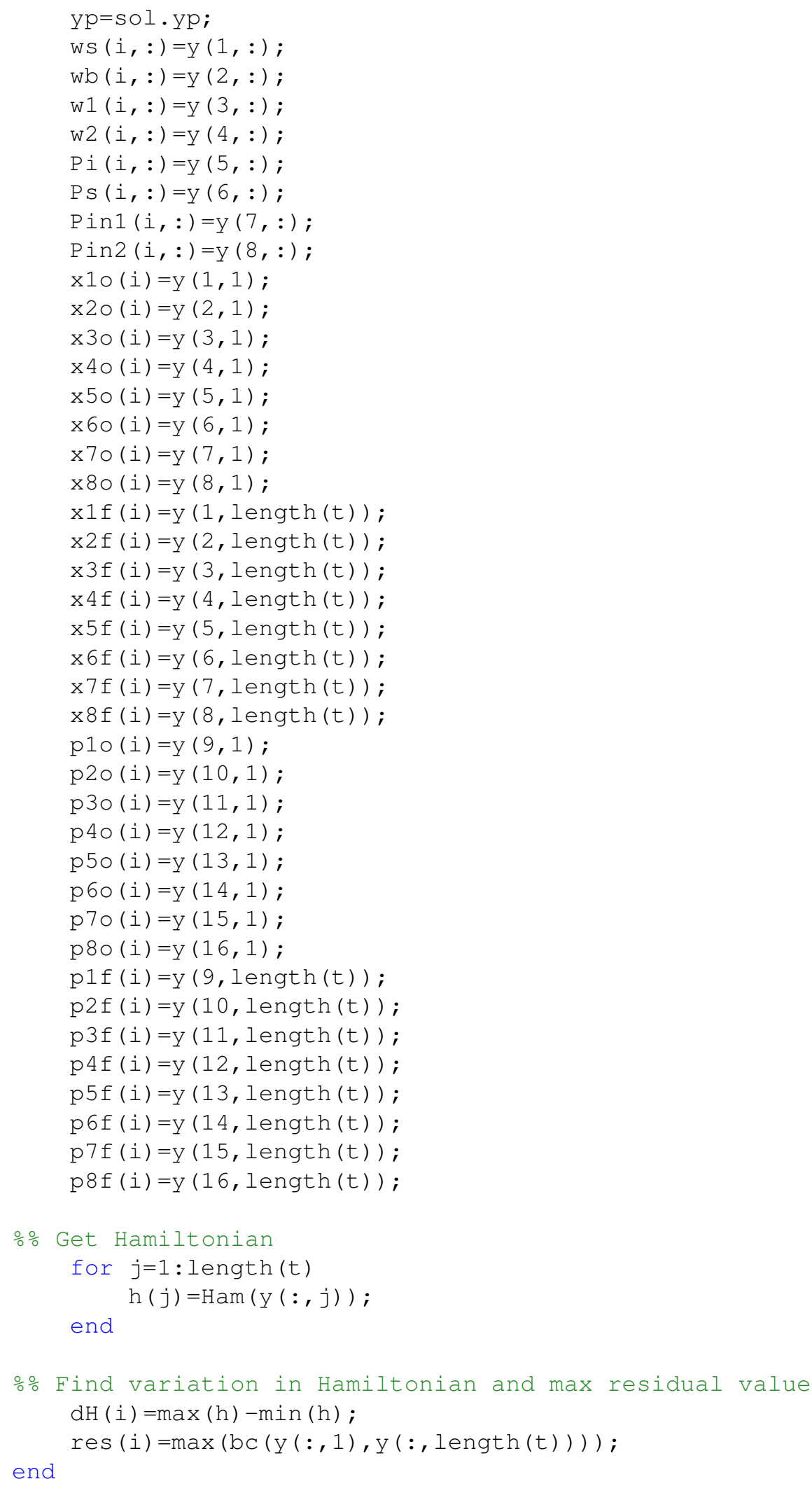




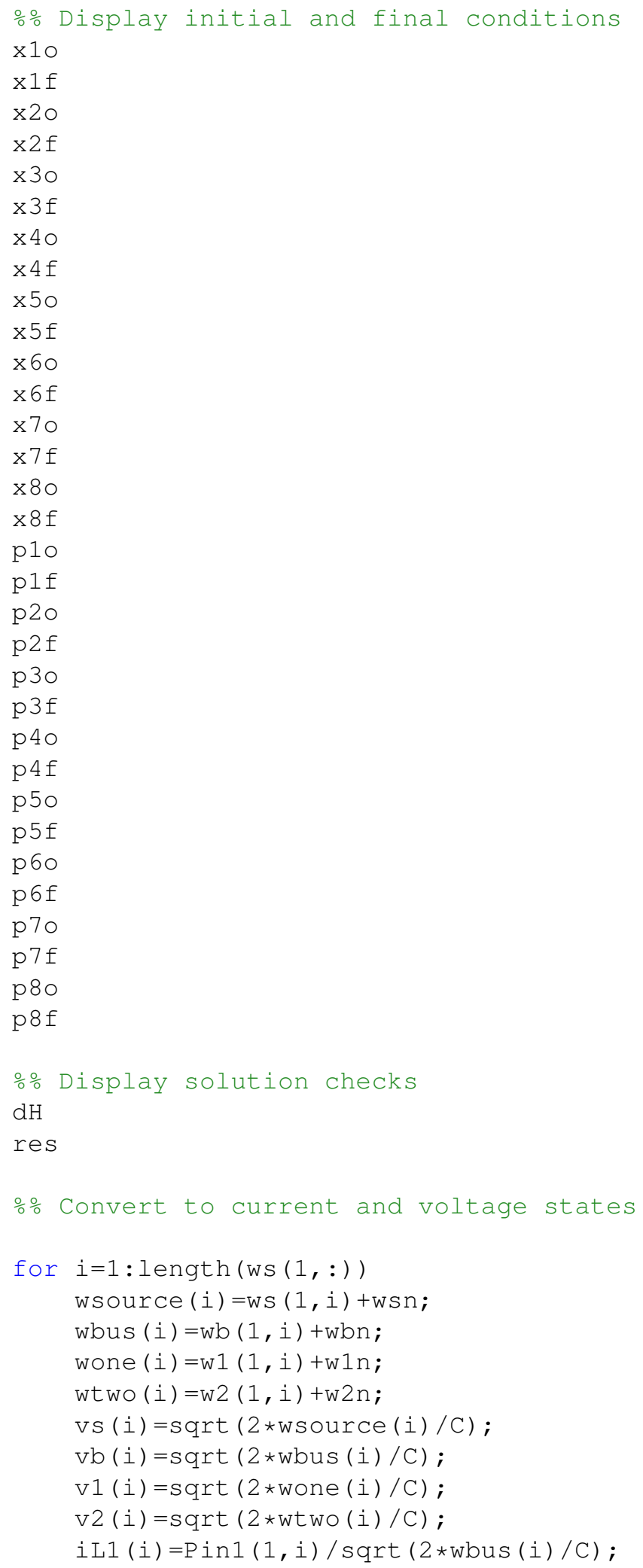




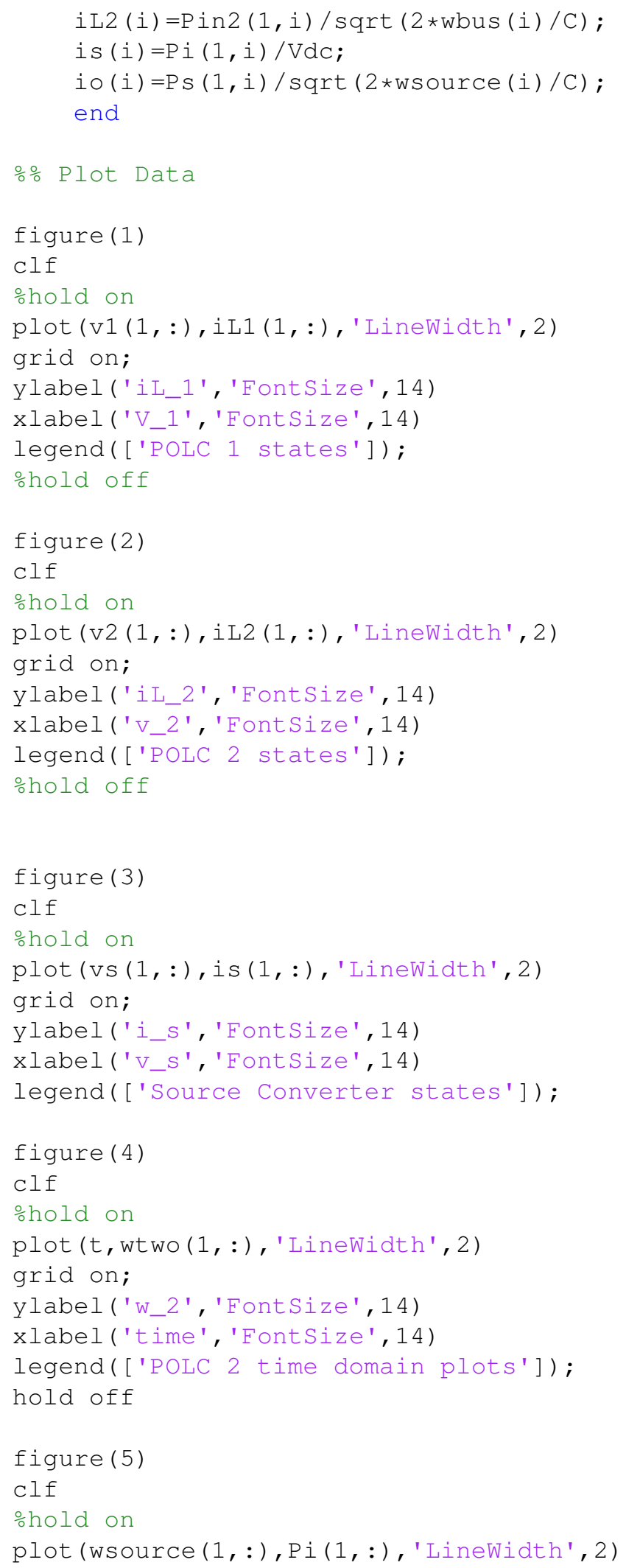




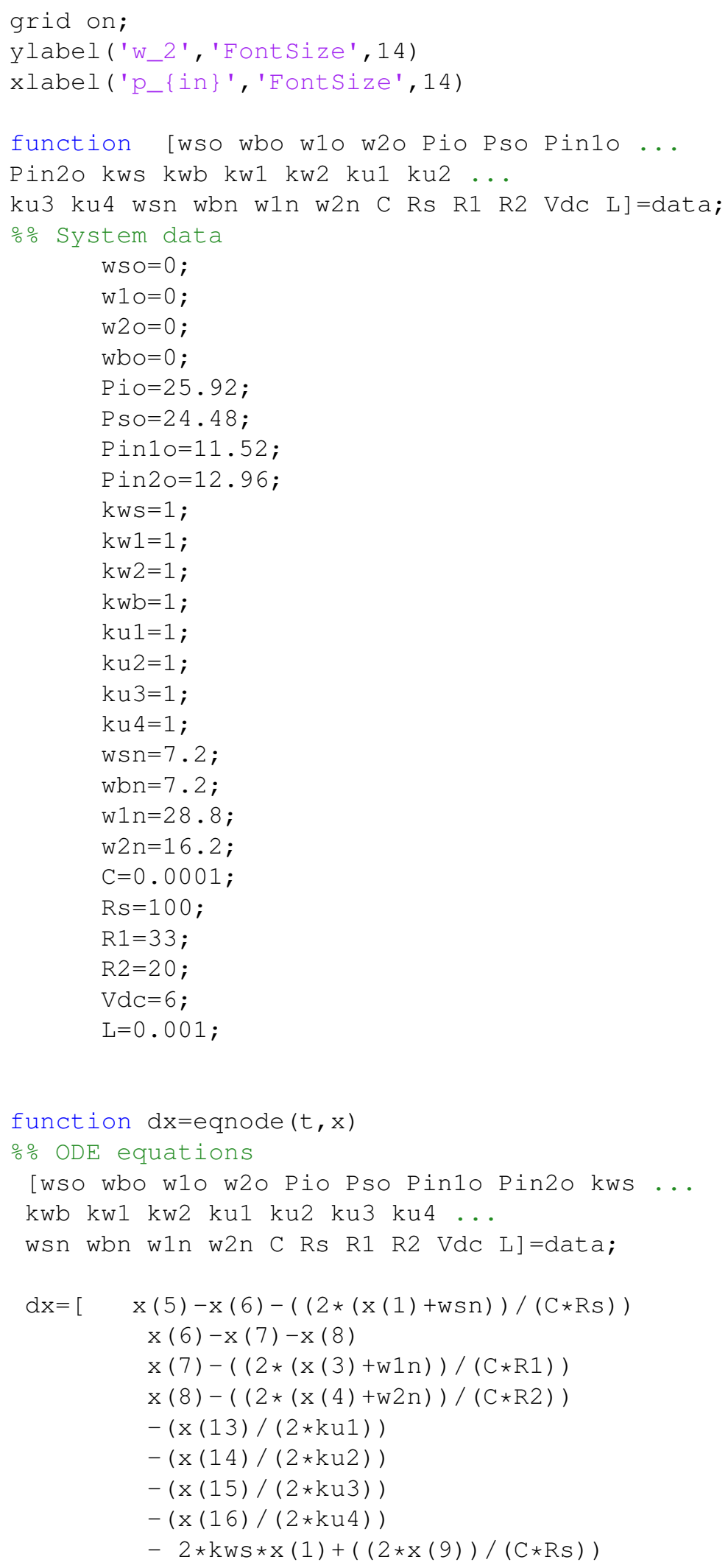




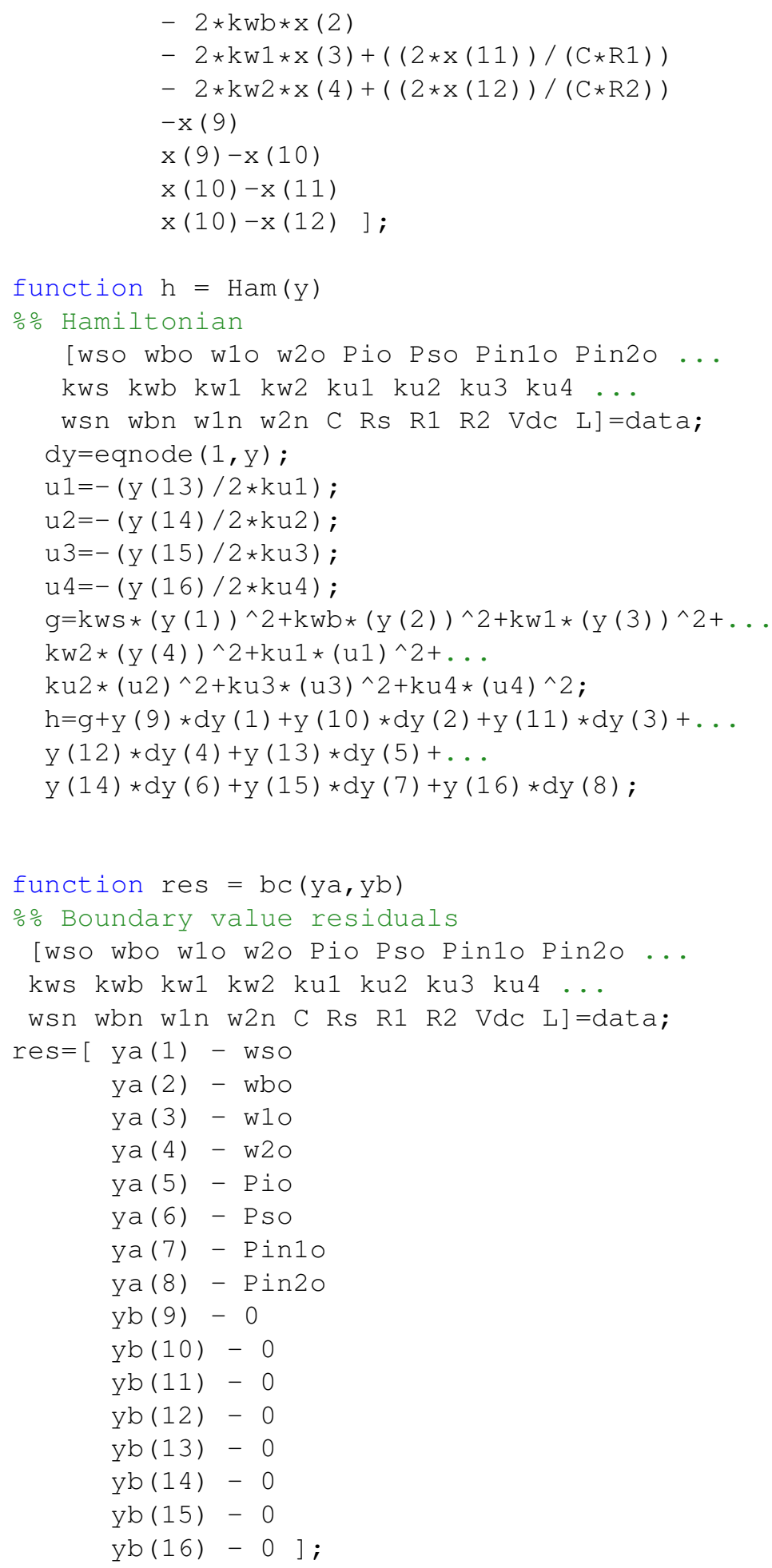




\section{A.2 MATLAB script for the solution of optimal control BVP for the dc-dc boost converter}

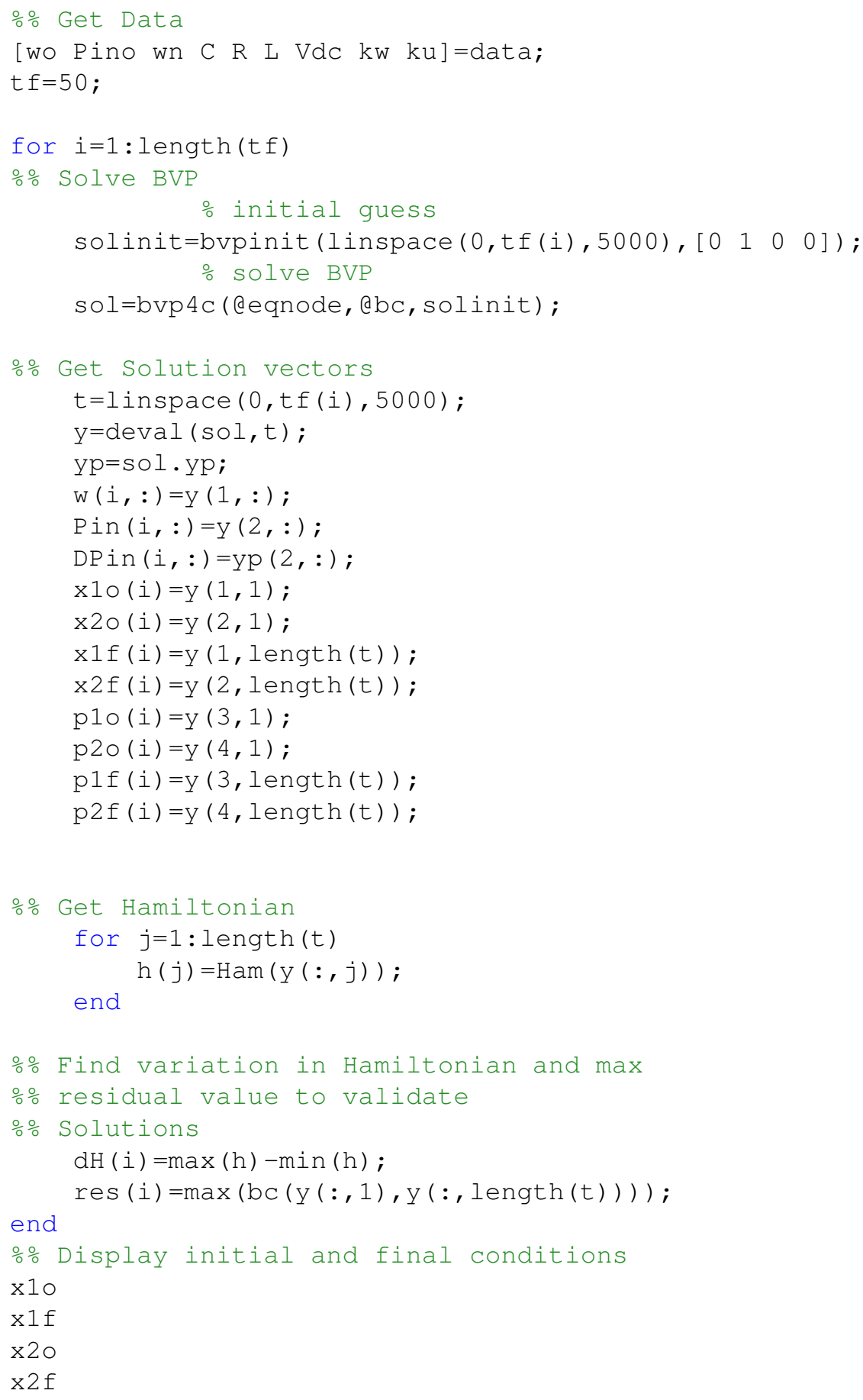




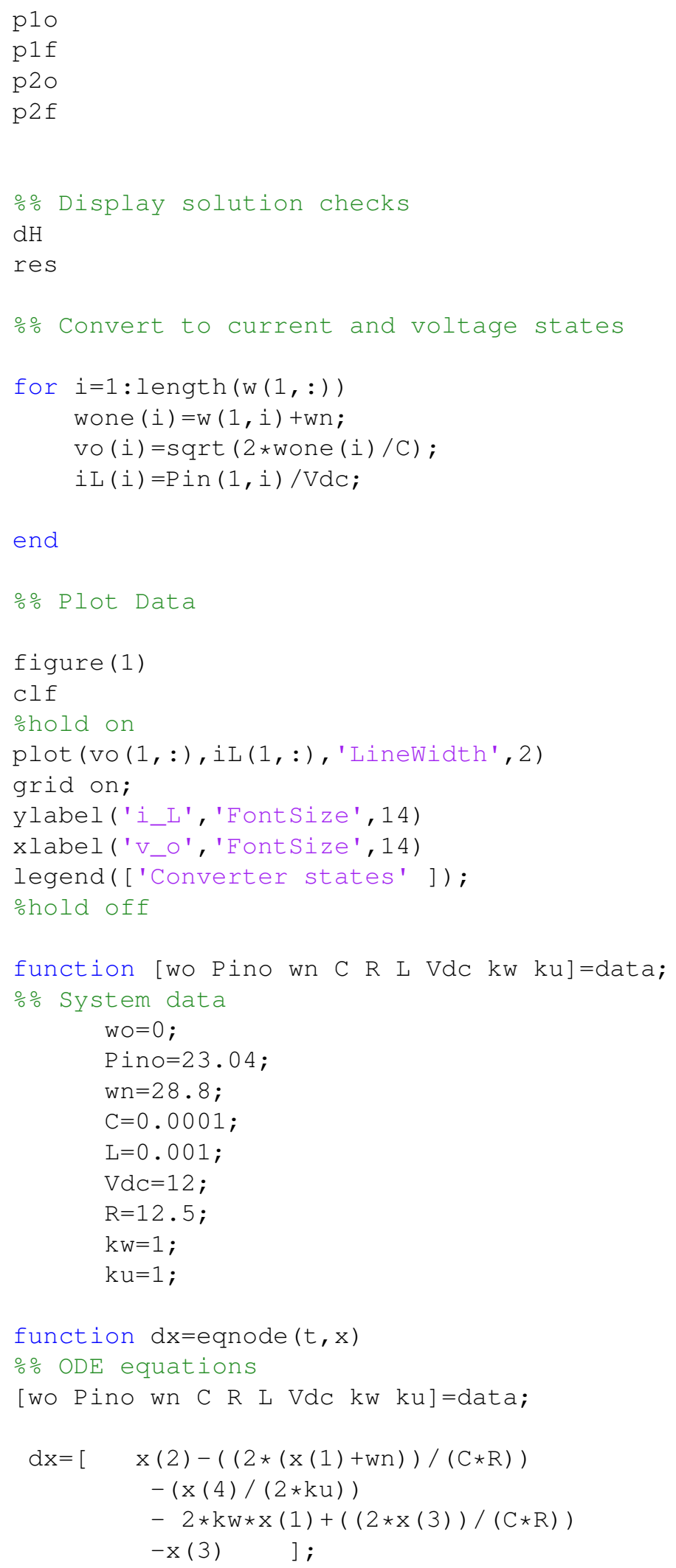




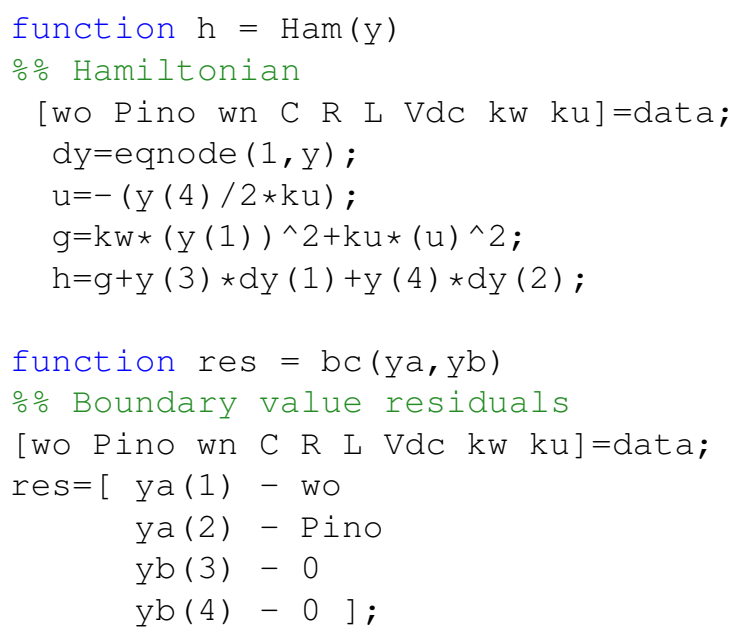

\section{A.3 MATLAB script and Simulink model for the control of the dc-dc boost converter example system for a straight line reference surface}

The MATLAB script for the control of the dc-dc boost converter example system for a straight line reference surface is shown below.

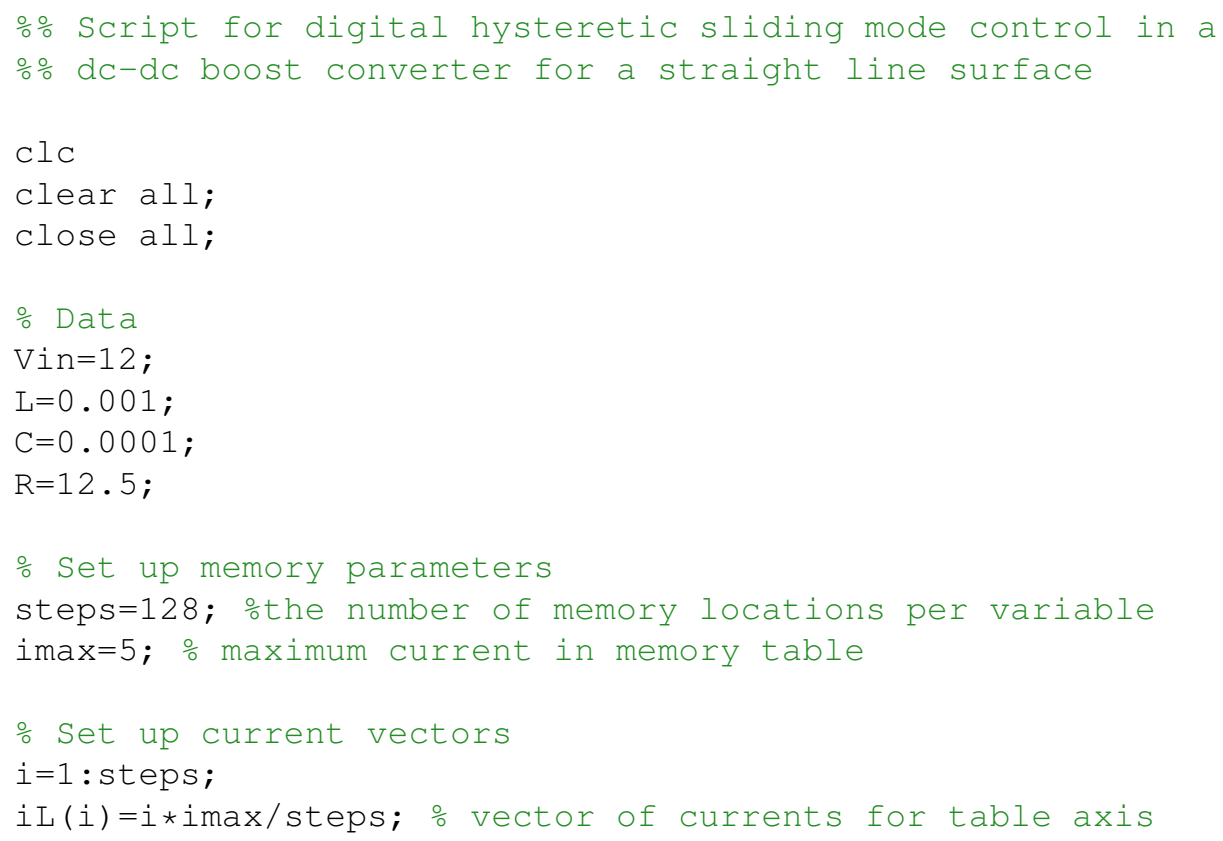




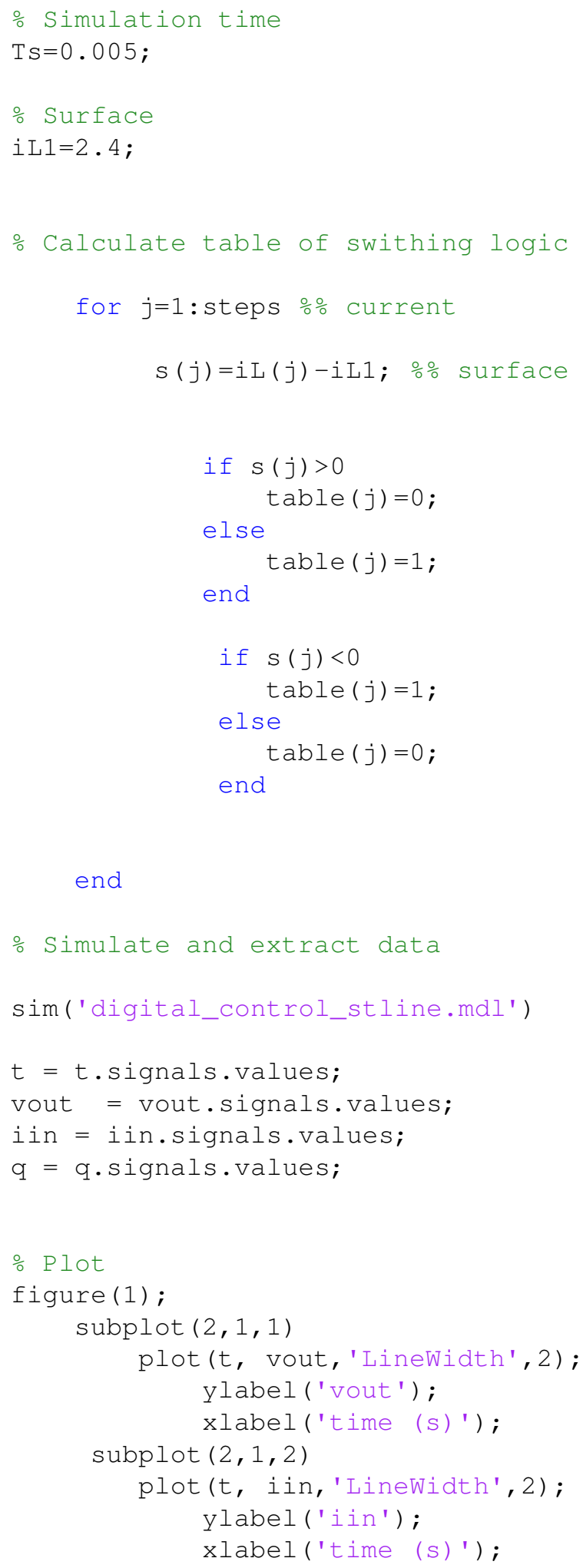




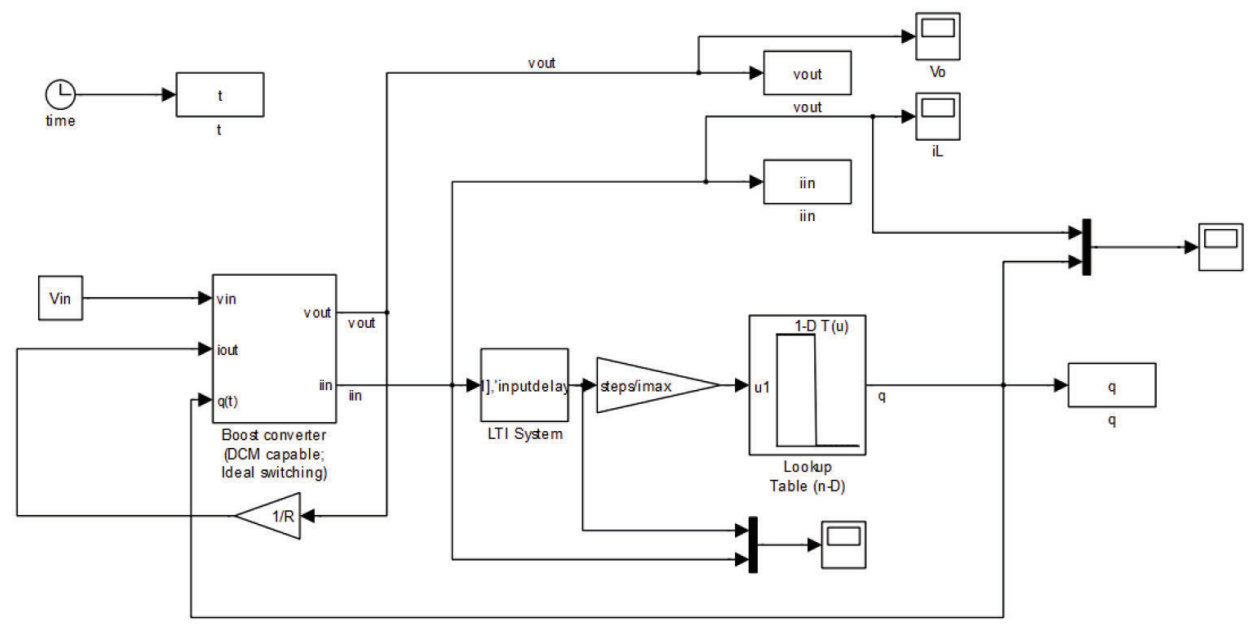

Figure A.1: Simulink model for the dc-dc boost converter example system for a straight line reference surface.

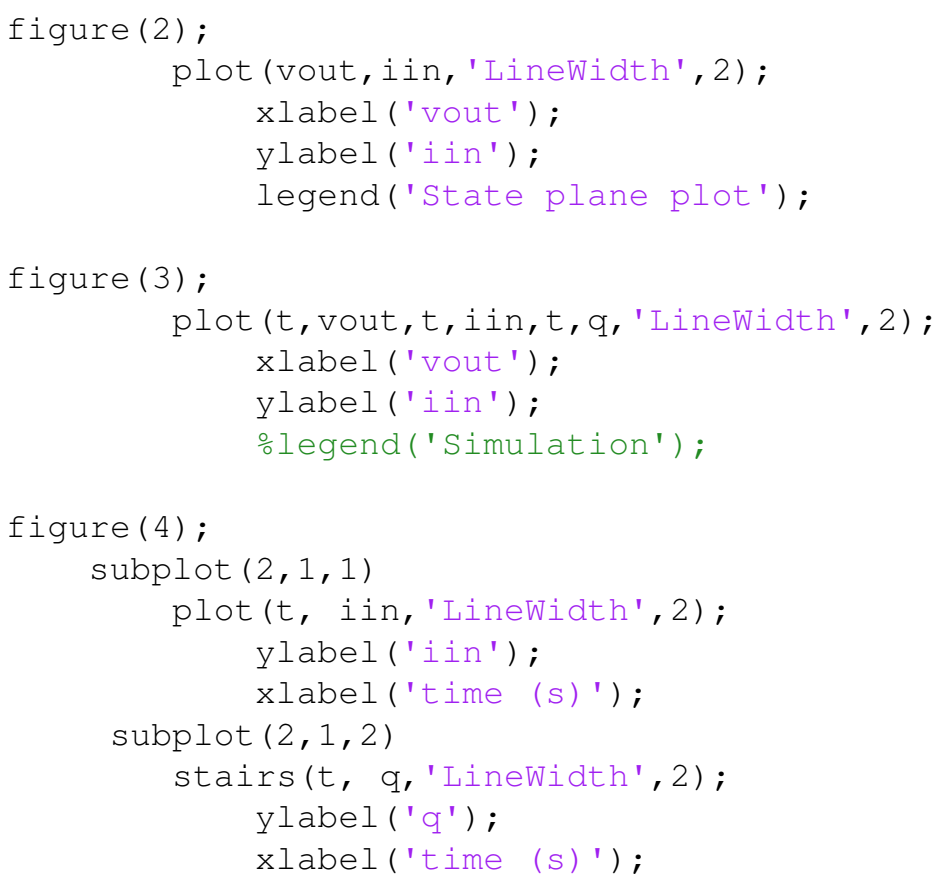

The Simulink model for the control of the dc-dc boost converter example system for a straight line reference surface is shown in Fig. A.1. 


\section{A.4 MATLAB script and Simulink model for the control of the dc-dc boost converter example system for a sloped linear reference surface}

The MATLAB script for the control of the dc-dc boost converter example system for a sloped linear reference surface is shown below.

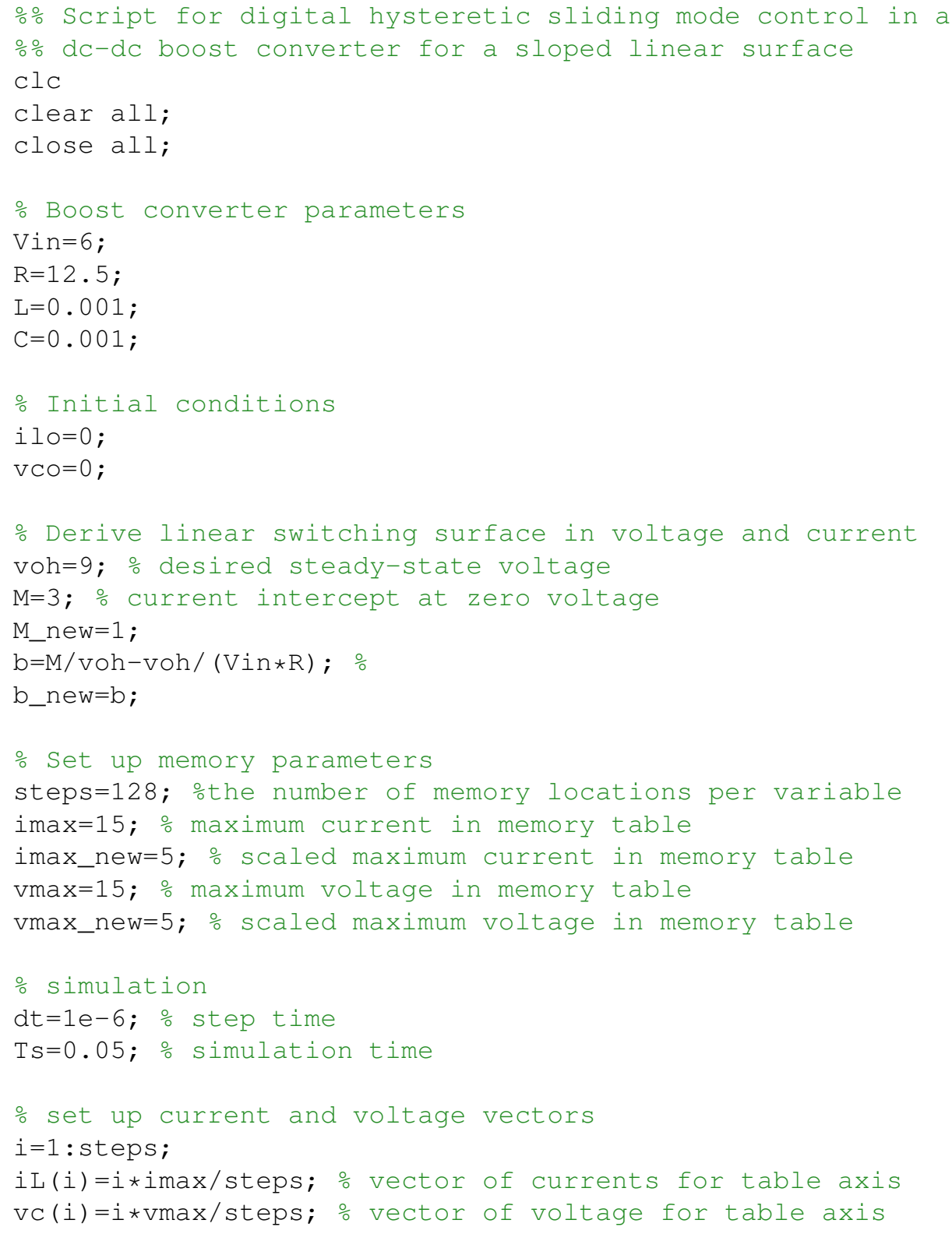




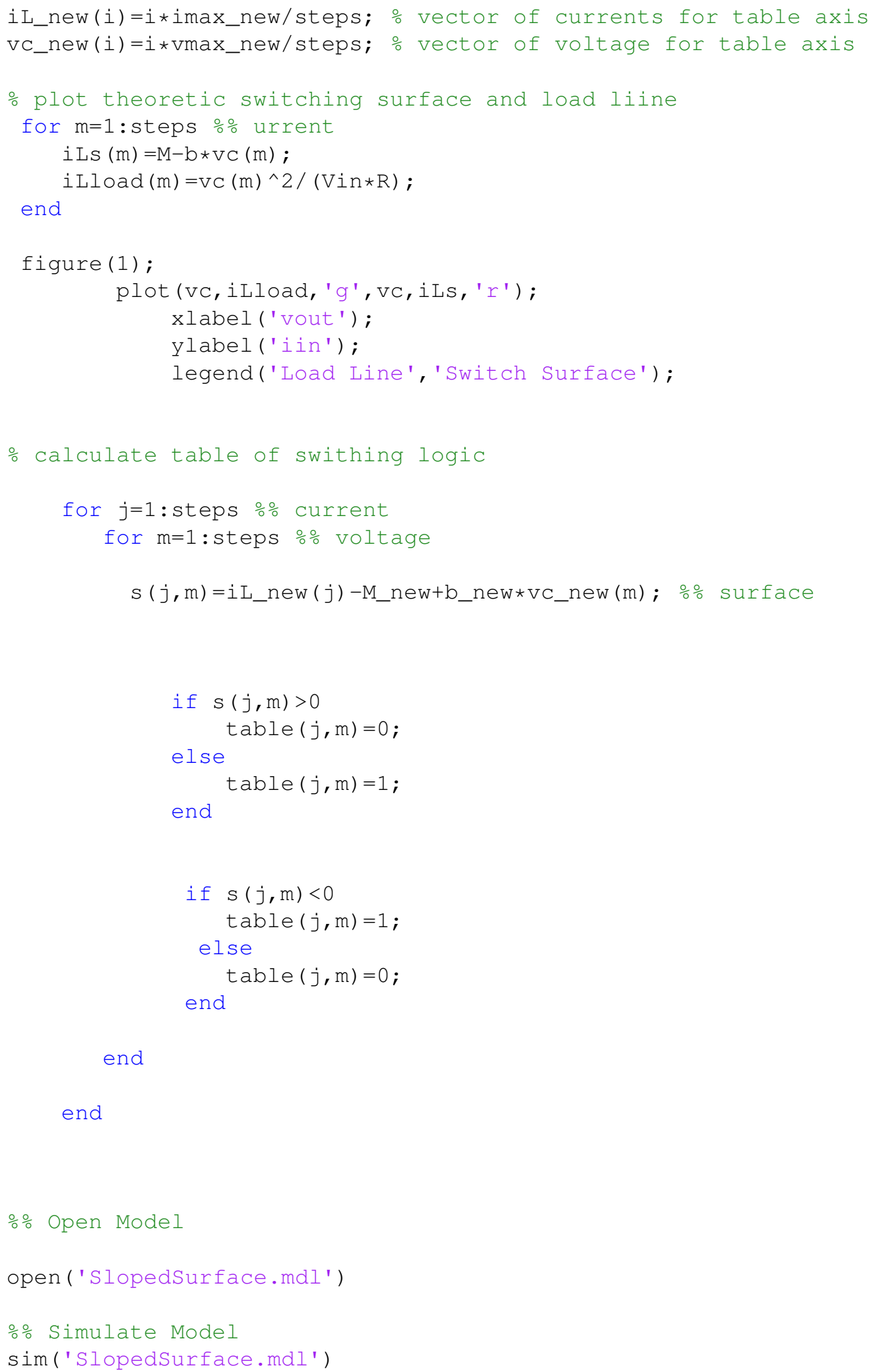




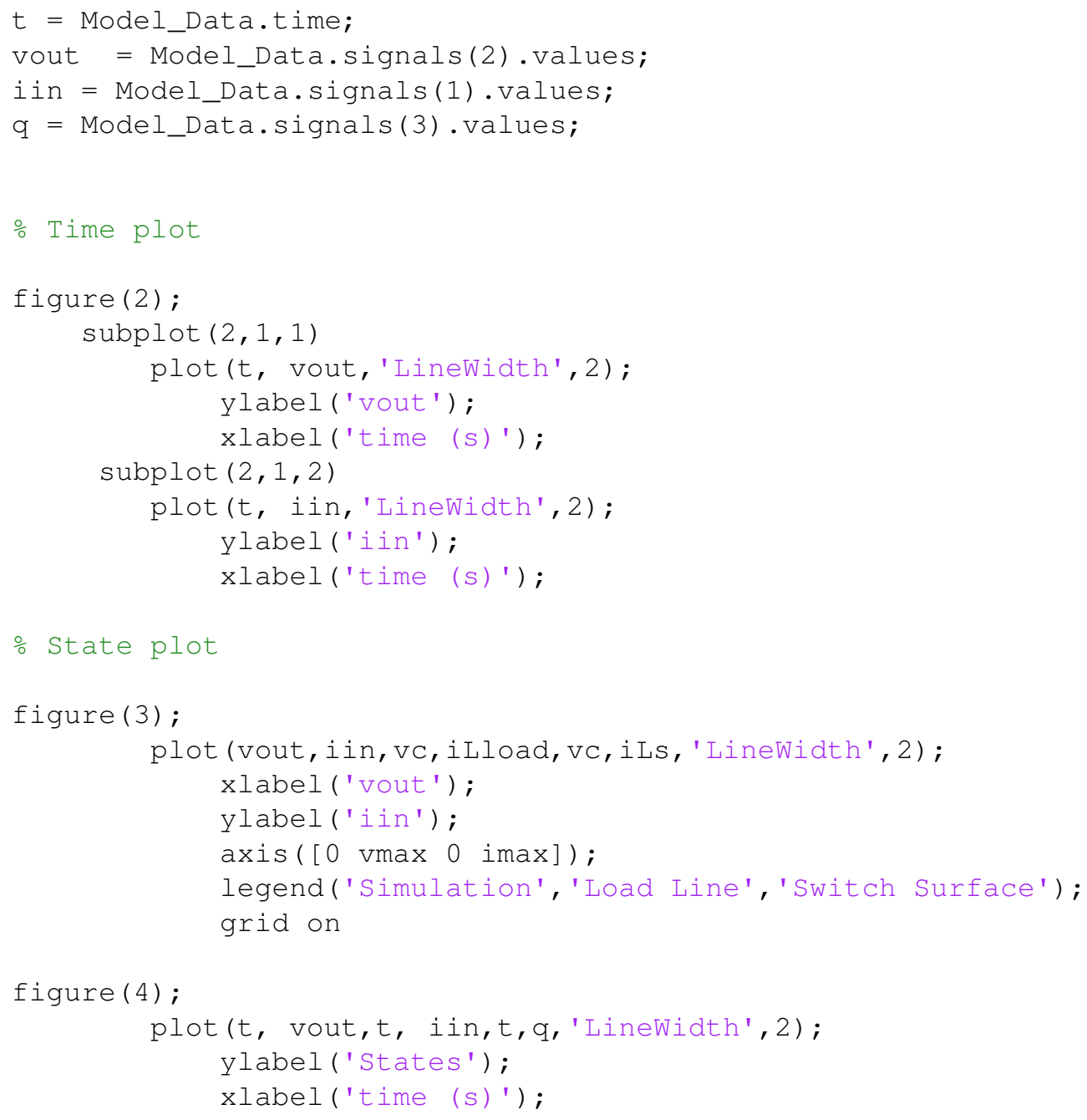

The Simulink model for the control of the dc-dc boost converter example system for a sloped linear reference surface is shown in Fig. A.2. 


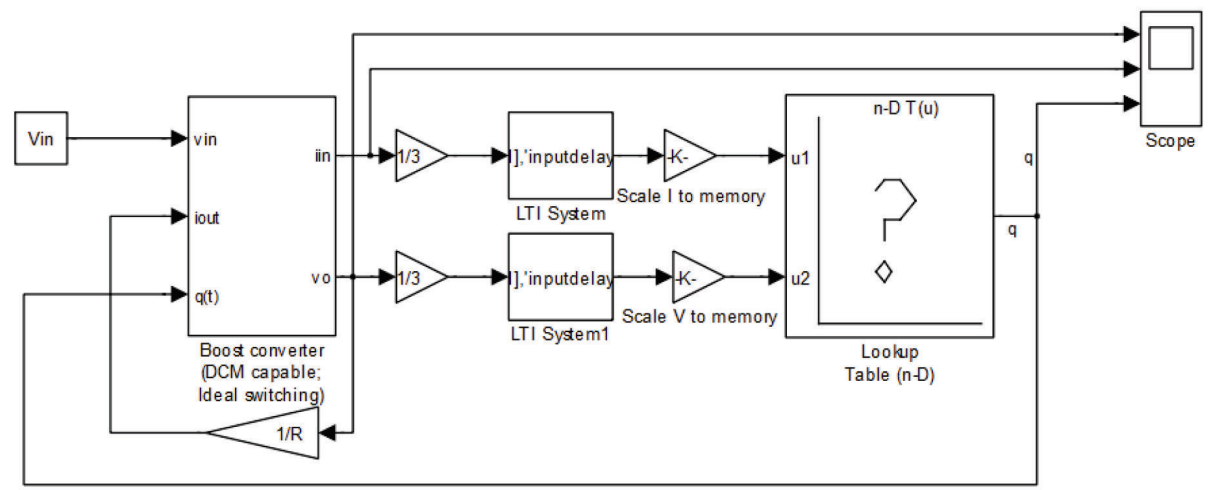

Figure A.2: Simulink model for the dc-dc boost converter example system for a sloped linear reference surface.

\section{A.5 MATLAB script and Simulink model for the control of the dc-dc boost converter example system for an optimal non-linear reference surface}

The MATLAB script for the control of the dc-dc converter example system for an optimal non-linear reference surface is shown below.

$\mathrm{ClC}$

clear all;

close all;

\% Boost converter parameters

Vin=12;

$\mathrm{L}=0.001 ;$

$\mathrm{C}=0.0001$;

$\mathrm{R} 1=25$;

$\mathrm{R} 2=12.5 ;$ 


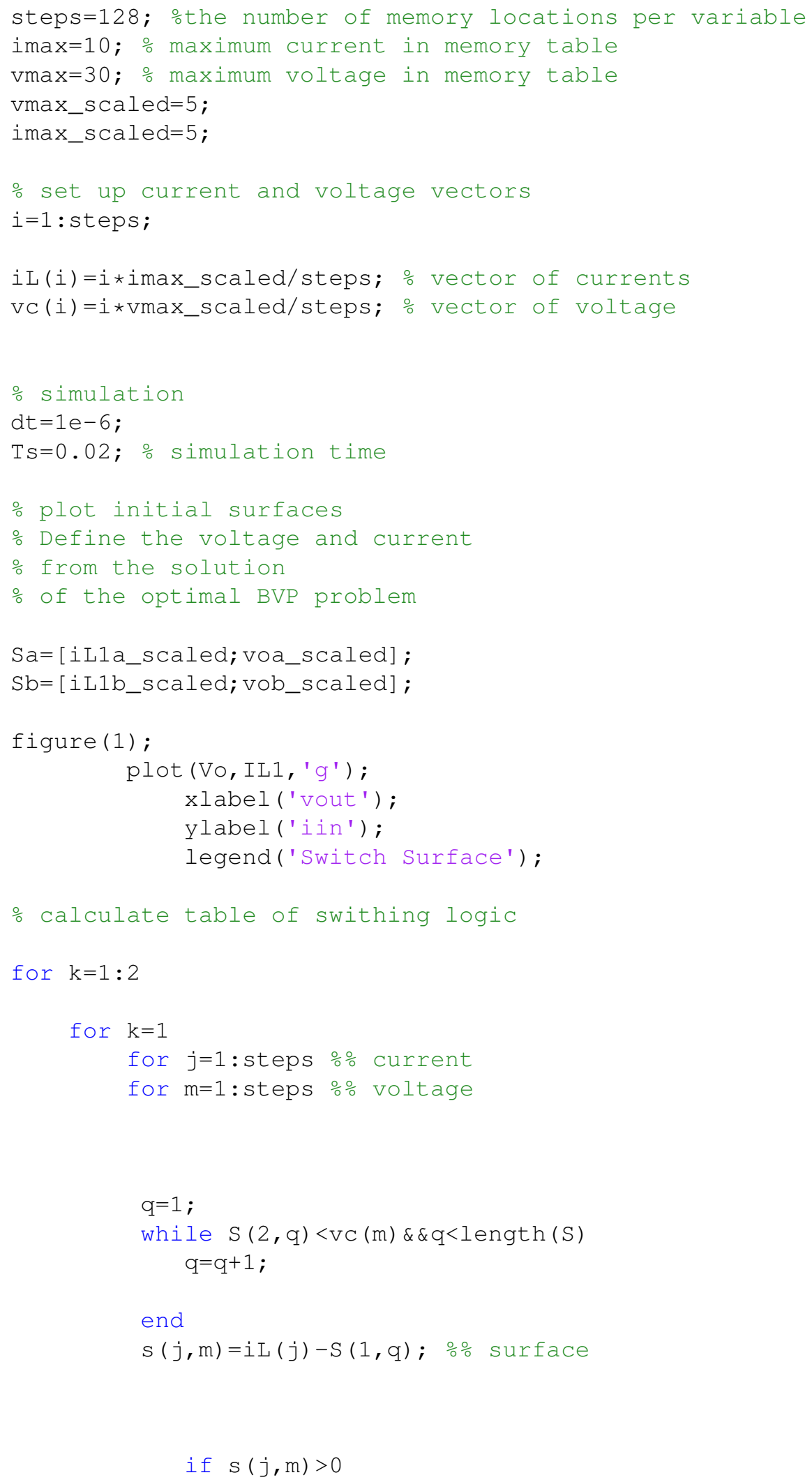




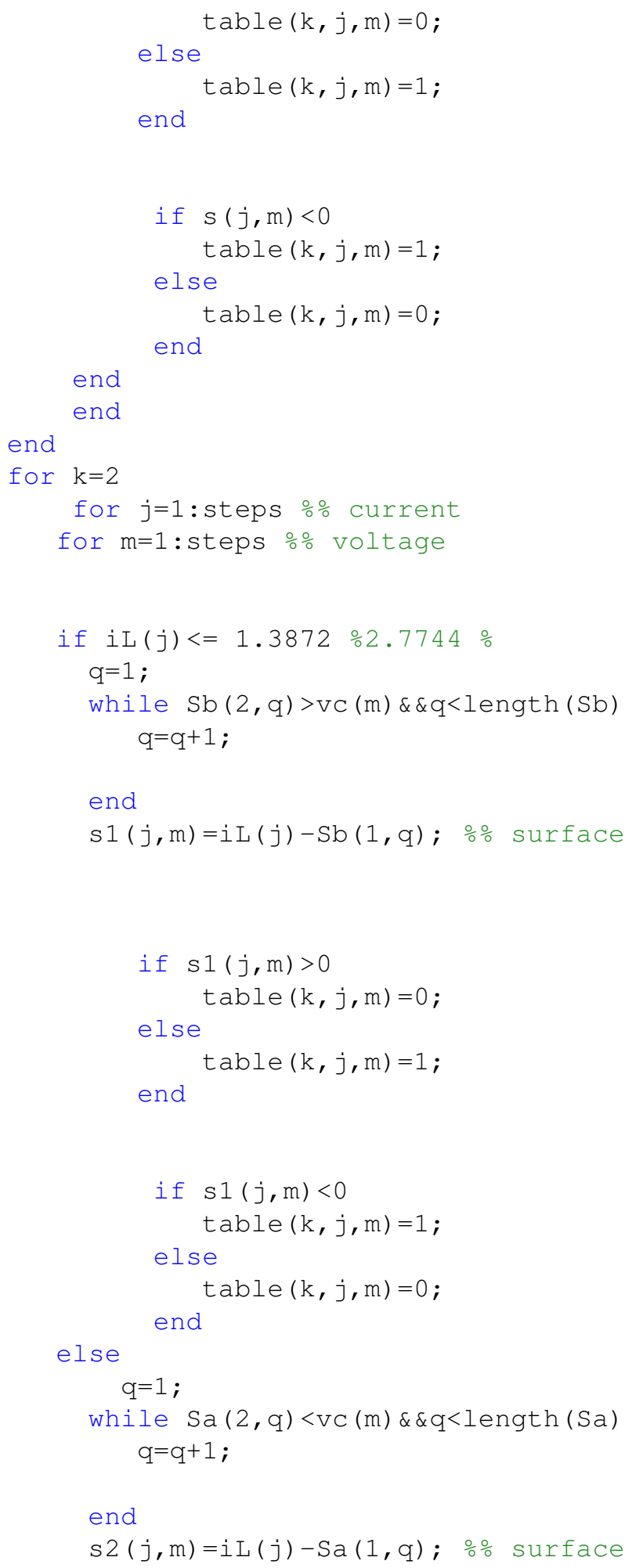




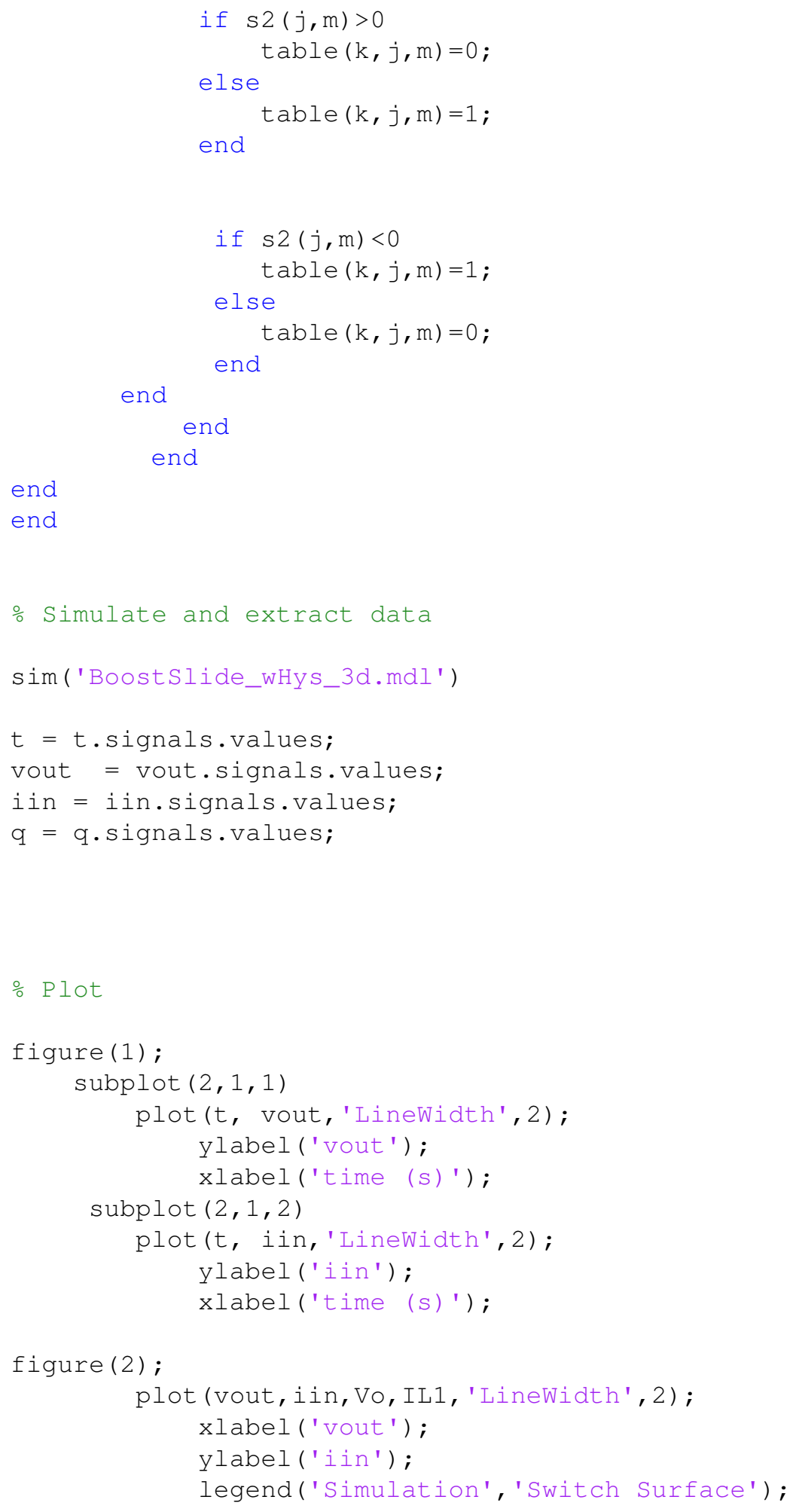




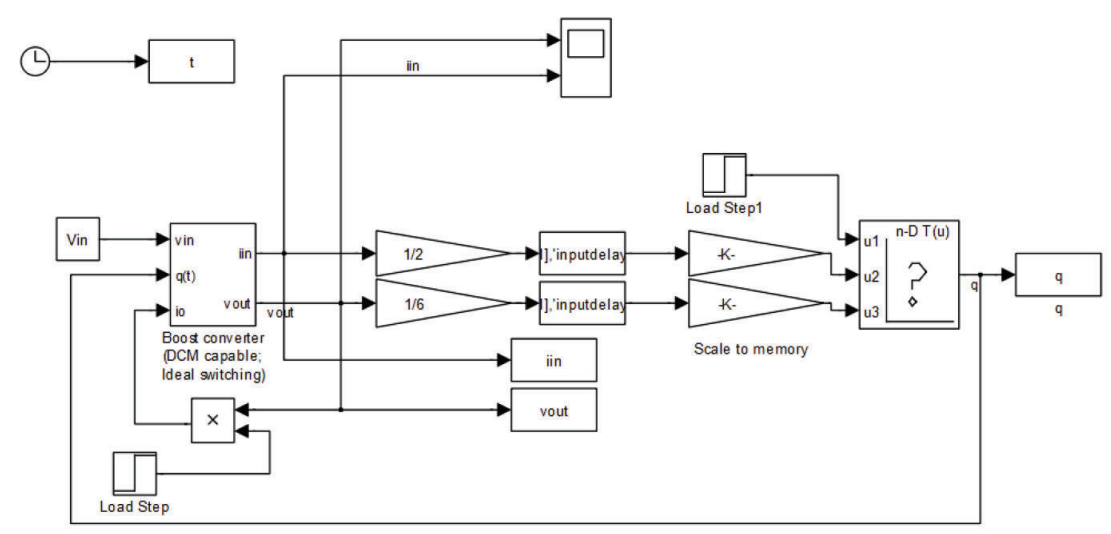

Figure A.3: Simulink model for the dc-dc converter example system for an optimal non-linear reference surface.

The Simulink model for the control of the dc-dc converter example system for an optimal non-linear reference surface is shown in Fig. A.3.

\section{A.6 MATLAB script and Simulink model for the control of the dc microgrid for step change in the loads}

The MATLAB script for the control of the dc microgrid for step change in the loads is shown below.

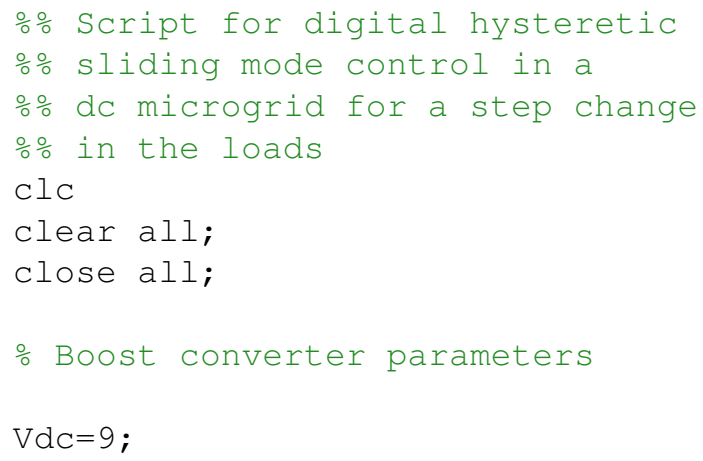




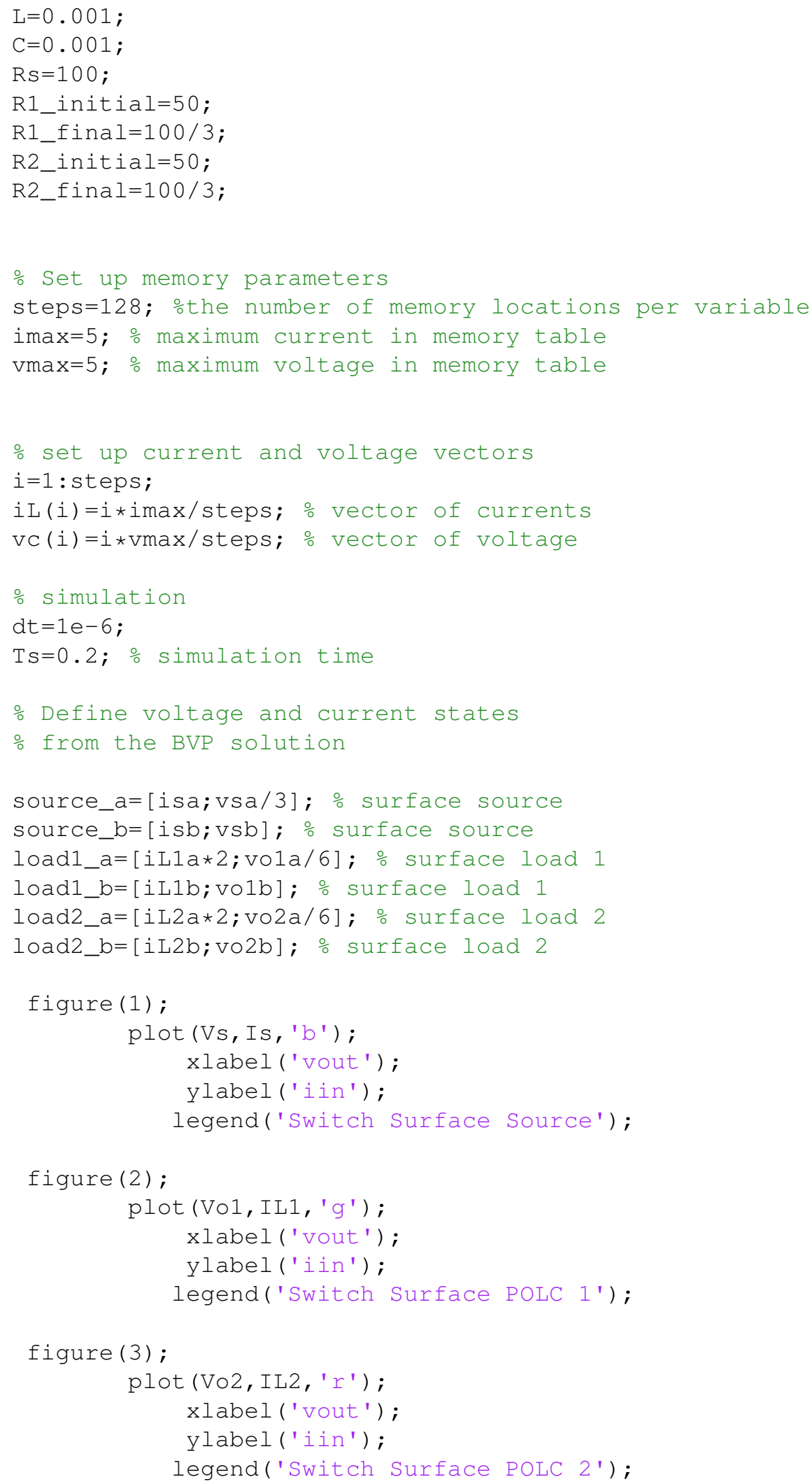




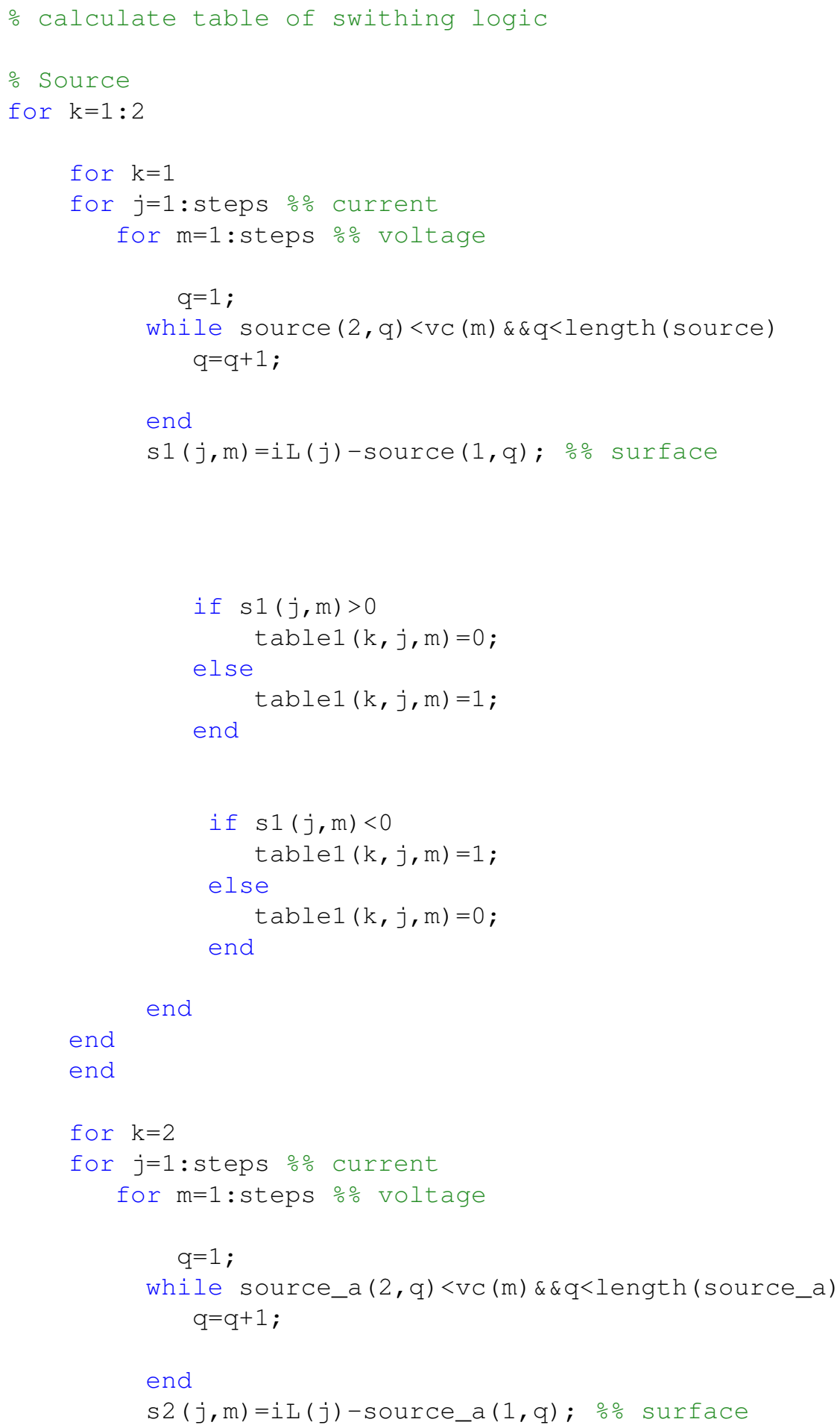




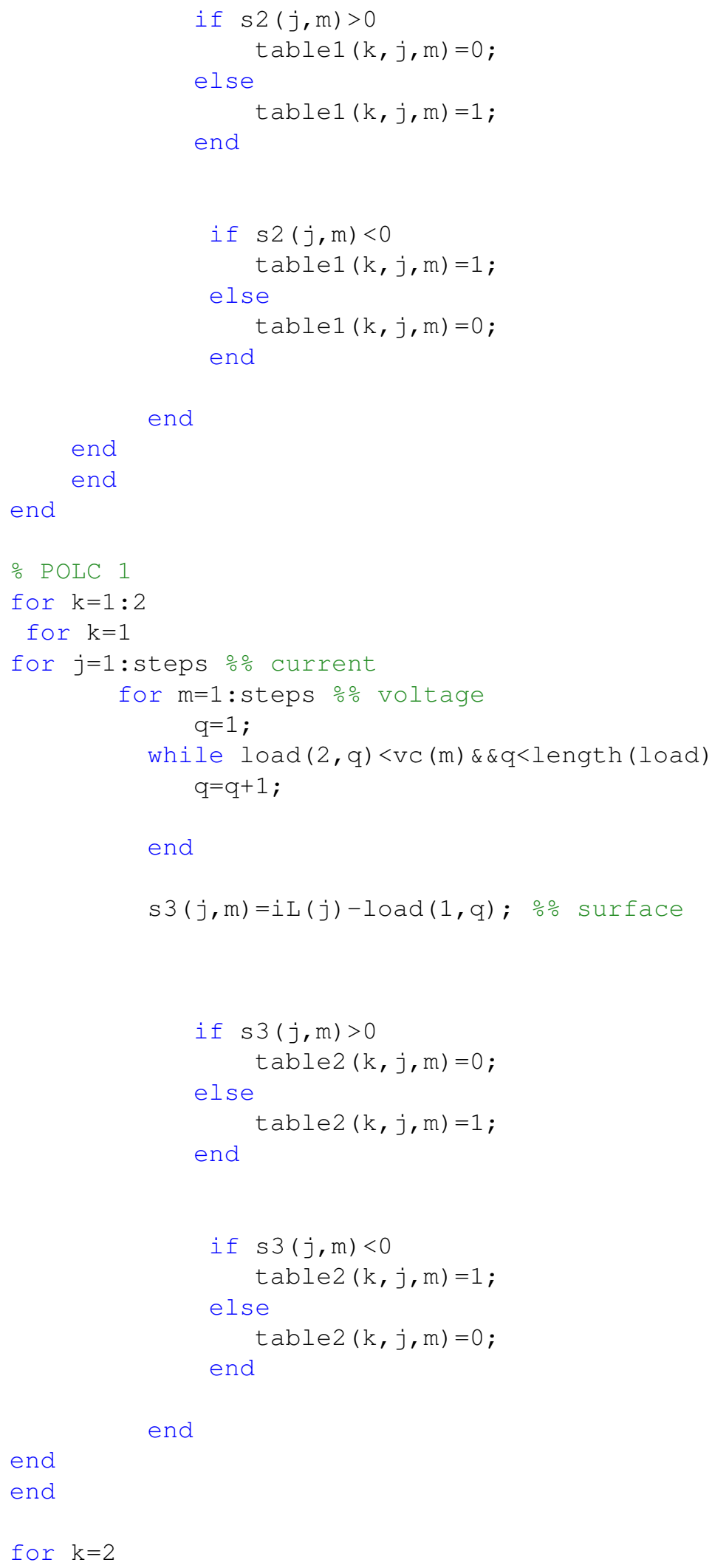




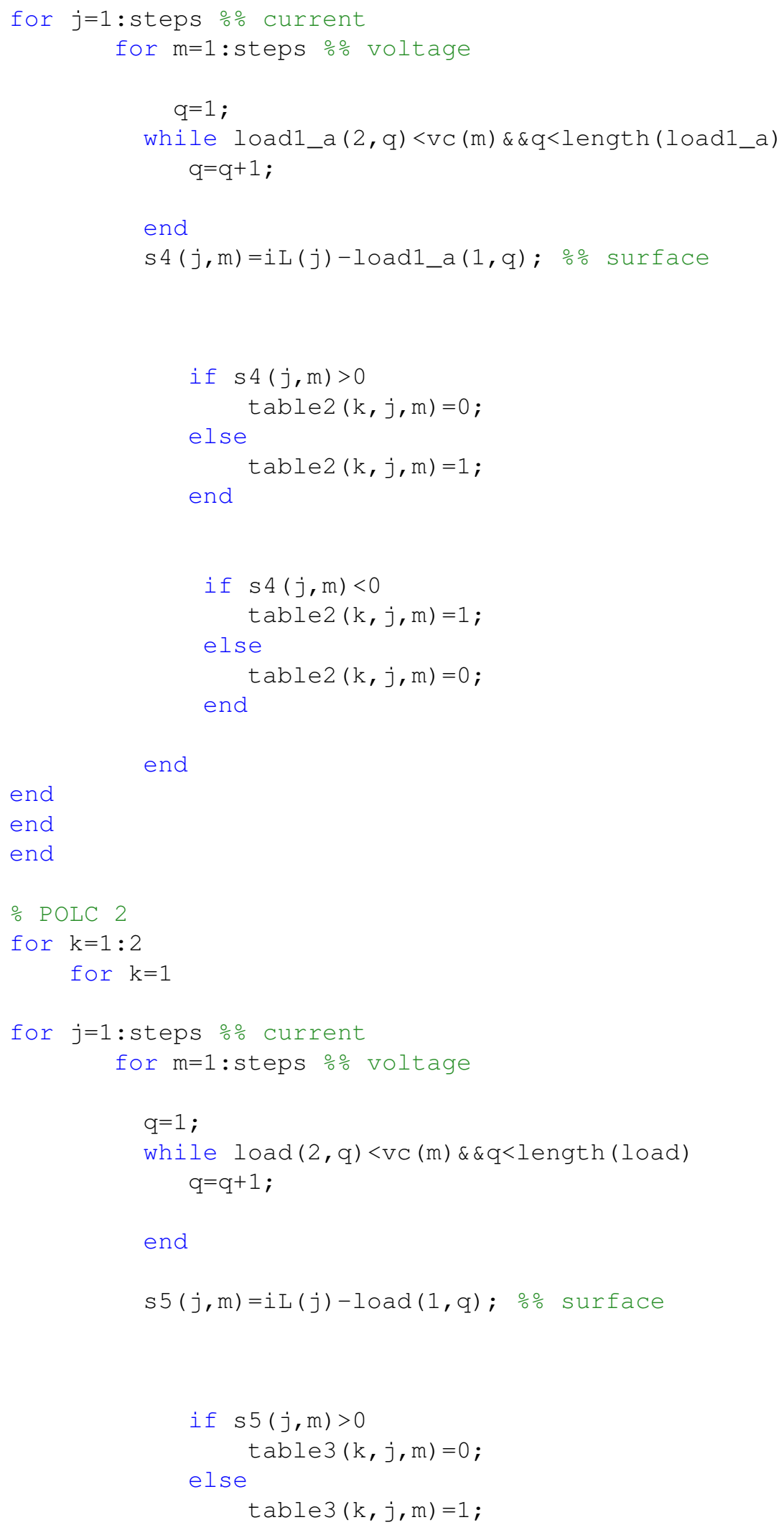




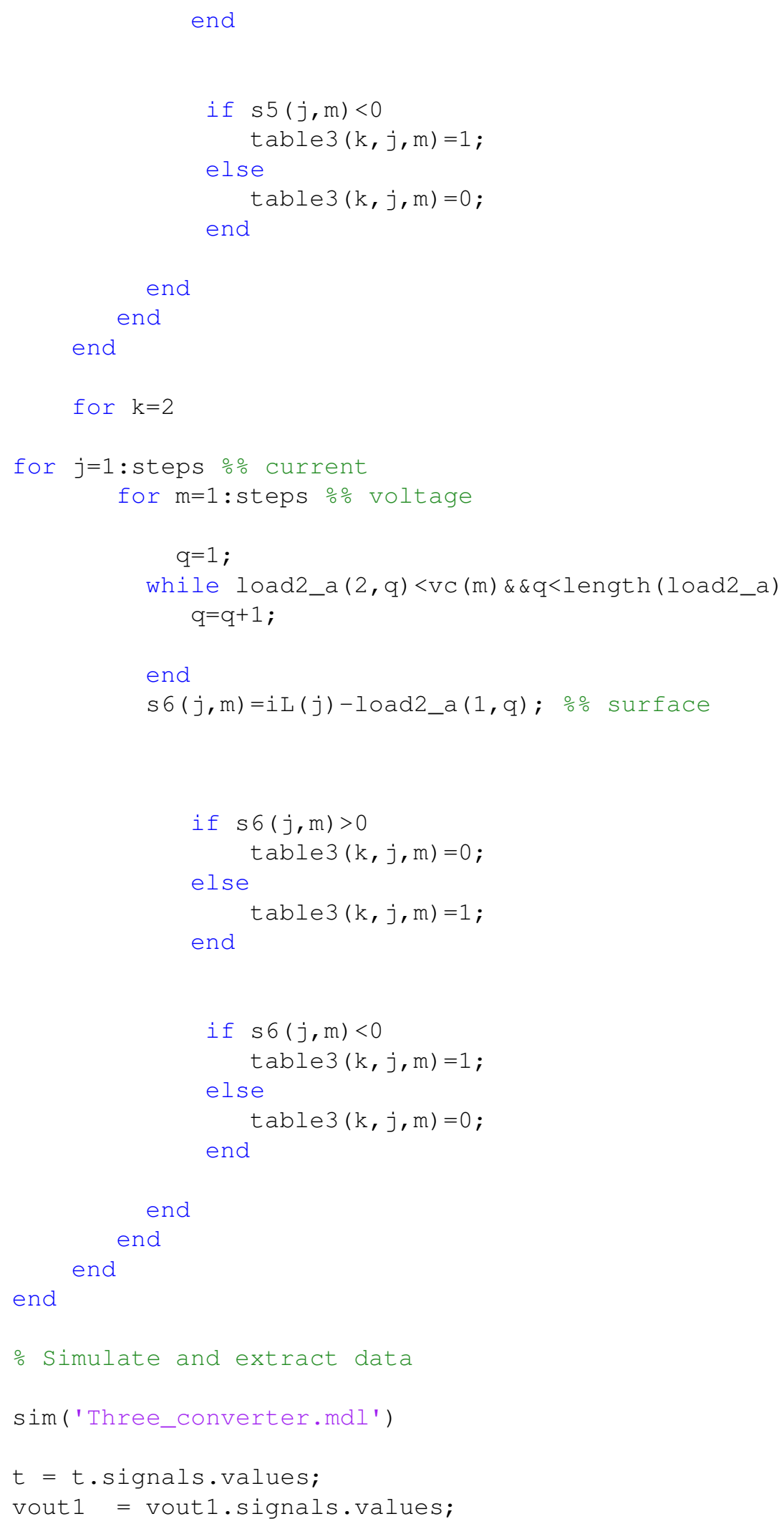




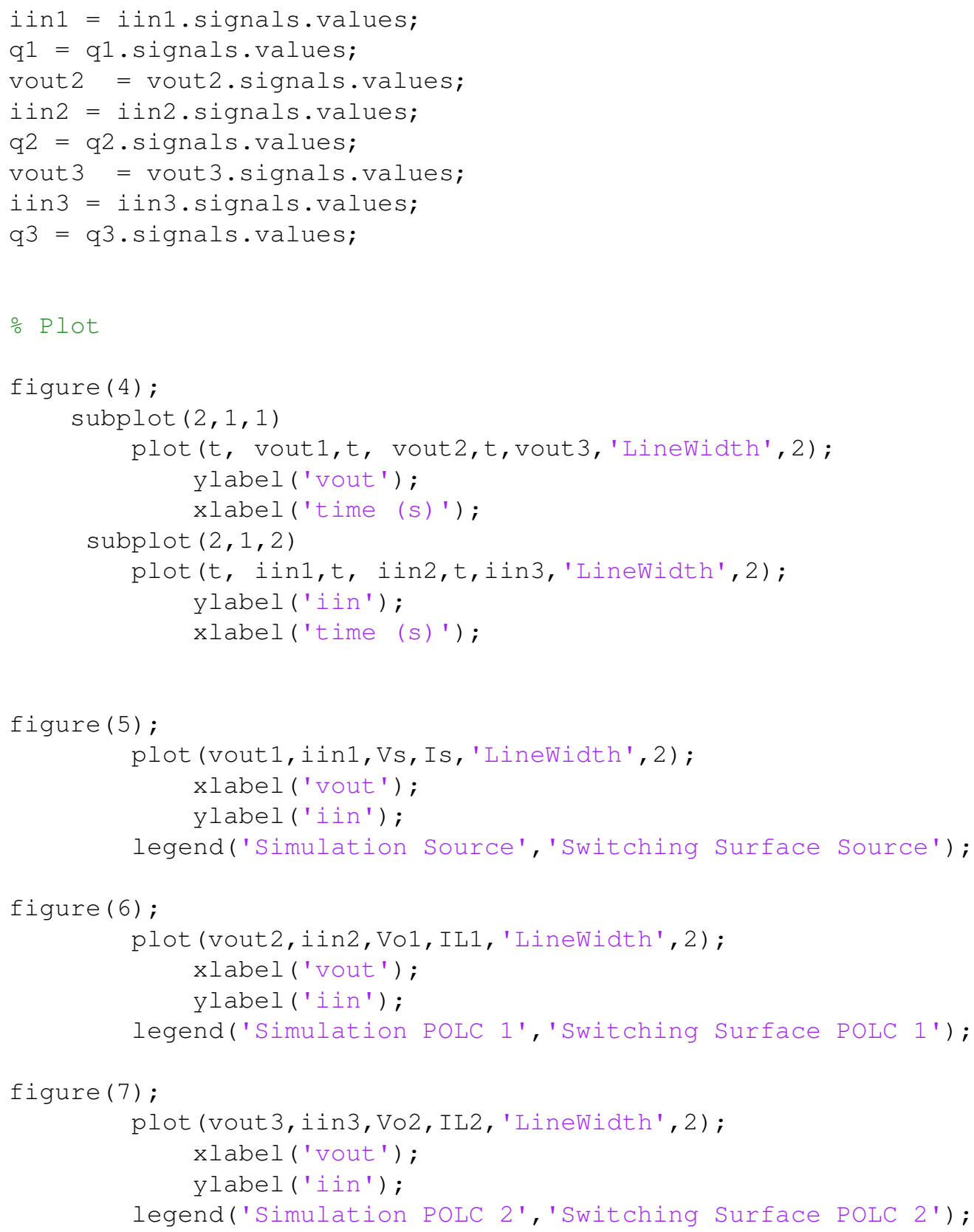

The Simulink model for the control of the dc microgrid for a step change in the loads is shown in Fig. A.4. 


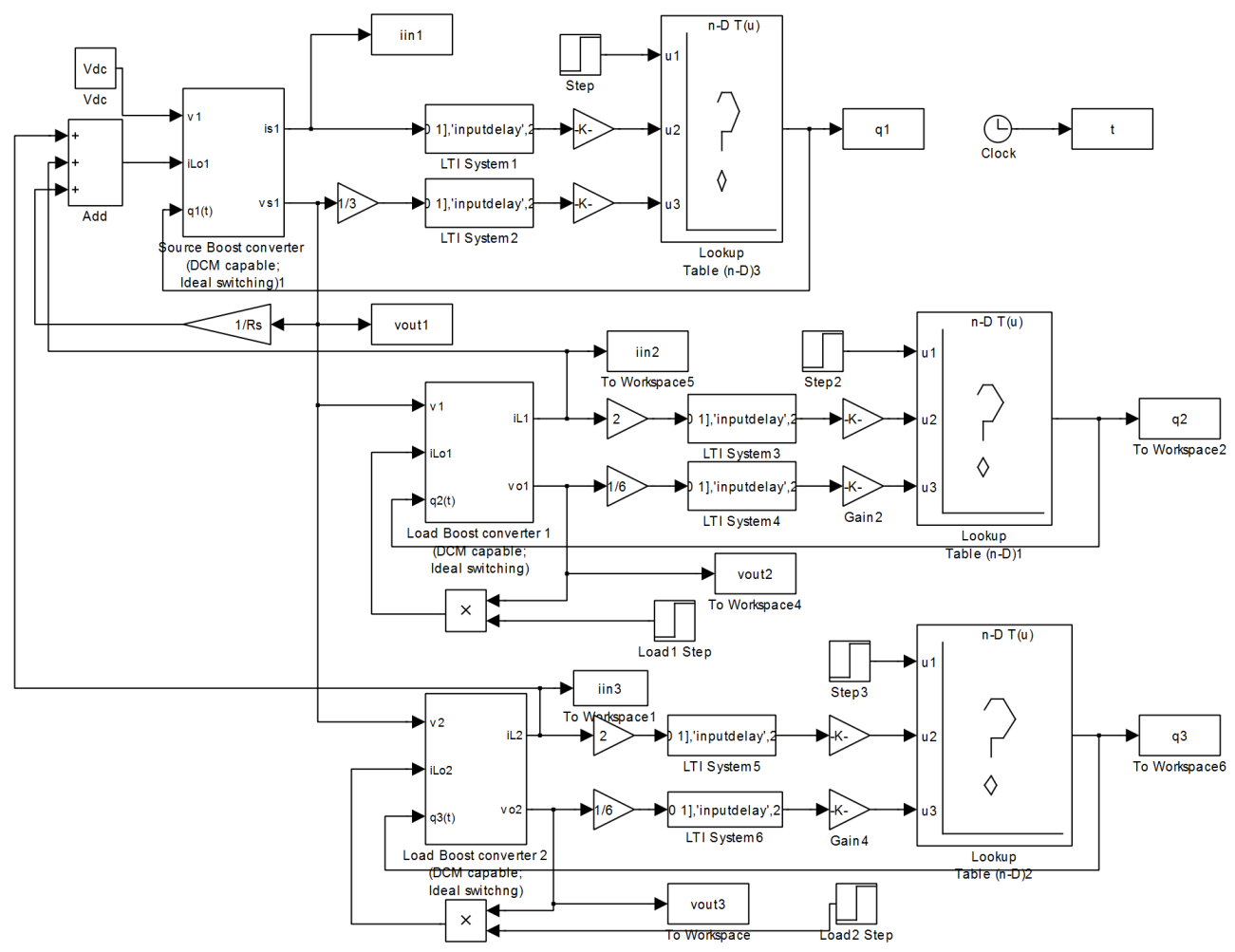

Figure A.4: Simulink model for the dc microgrid for a step change in the loads. 